\title{
Testes de Sobrevivência Acelerados: \\ Uma Análise Bayesiana \\ do Modelo de Eyring
}

\author{
Francisco Louzada Neto
}

\author{
Orientação: \\ Prof.' Dr. Jorge Alberto Achcar
}

Dissertação apresentada ao Instituto de Ciências Matemáticas de São Carlos, da Universidade de São Paulo, como parte dos requisitos para obtenção do título de mestre em Ciências de Computação e Matemática Computacional.

USP - São Carlos

1991 
À minha esposa, Claudia Ao nosso Nenê Aos meus pais, Waldemar e M. Helena 


\section{Agradecimentos}

A conclusão dessa dissertação não seria possivel sem o auxílio de algumas pessoas que contribuíram diretamente no estudo de Testes de Sobrevivência Acelerados, à quais expresso os mais sinceros agradecimentos.

Ao Prof. Dr. Jorge Alberto Achcar por me orientar neste trabalho com sua valiosa dedicação e segurança, participando ativamente do estudo, incentivando-me em todo o decorrer da pesquisa.

Ao Prof. Dr. Josemar Rodrigues pelo incentivo e apoio dado no início do meu curso de Pós-Graduação e pelas valiosas sugestões e comentários dados a este trabalho.

Ao Prof. Dr. Emanoel Pimentel Barbosa pelas oportunas discussões e sugestões sobre o tema em questão.

Também agradeço à Profạ Dra Maria Creusa S. Galvão Leite pelas sugestões apresentadas quando da realização do meu exame de qualificação; ao Prof. José Albertino R. Rodrigues (in memorian) pelo apoio e incentivo ao meu ingresso na Pós-Graduação; aos Colegas e Funcionários do Departamento de Estatística da Universidade Federal de São Carlos (UFSCar) pela acolhida e camaradagem durante a elaboração deste trabalho; aos Professores, Funcionários e Colegas da PósGraduação pelo excelente convivio; ao Conselho Nacional de Desenvolvimento Científico e Tecnológio (CNPq) pelo apoio financeiro parcial; a UFSCar pela concessão do afastamento parcial para a realização dos meus estudos; e finalmente ao Luciano Cesário e Carmen Lúcia Pagadigorria pelo excelente trabalho de digitação dos manuscritos. 


\section{Resumo}

Nesta dissertação, considerando Testes de Sobrevivência Acelerados, desenvolvemos uma análise Bayesiana, do modelo de Eyring com uma variável estresse, $\mathrm{e}$ é considerada sua extensão para uma relação estresse-resposta geral, onde os modelos mais utilizados em exeperimentos acelerados com uma variável estresse são casos particulares. Também, o modelo de Eyring generalizado é analisado sob o enfoque Bayesiano. Consideramos, nestas análises, tanto uma distribuição Exponencial para os tempos de sobrevivência dos componentes, quanto uma distribuição de Weibull, dados sob um esquema de censura de tipo II e densidades a priori não-informativas de Jeffreys para os parâmetros envolvidos. 0 método de Laplace é usado para aproximarmos as densidades a posteriori de interesse quando não conseguimos achar suas soluções analíticas explícitas. Além disso, a precisão dos valores aproximados é estudada e relacionada com o uso de uma parametrização adequada. Também, achamos a densidade preditiva para uma observação futura, em um nível especificado de estresse, a qual é usada para desenvolvermos testes de controle de qualidade. 


\begin{abstract}
Accelerated life tests are considered in this dissertation. A Bayesian analysis of the Eyring model is developed and its extension on to a general stress-response relationship is considered, where the most used models for accelerated experiments, with one stress variable are obtained as particular cases. Also, the generalized Eyring model is analysed under a Bayesian view point. We consider in the analysis an Exponential and Weibull distribution for the units lifetime, a type II censored data and a non-informative Jeffreys priors for the parameters involved. The Laplace's method is used to approximate the posterior densities when we cannot find analytical solutions explicitly. The precision of the approximated values otained are studied and then related to the use of adequated parametrizations. We also find the predictive density for a future observation in a specified stress level, which is used to develop quality control tests.
\end{abstract}




\section{Terminologia e Notação}

Variáveis aleatórias são representadas por letras maiúsculas e suas realizações por minúsculas.

A função densidade de probabilidade de uma variável aleatória é representada por $f($.$) , as funções de verossimilhança dos parâmetros envolvidos por L($.$) , as$ densidades a priori dos parâmetros envolvidos por $\pi($.$) , as densidades a posteriori$ por $\pi(. / d a d o s)$ e a densidade preditiva de uma observação futura por $f(. /$ dados $)$.

Letras gregas iniciam os parâmetros dos modelos.

Em geral, constantes são representadas por letras minúsculas.

Vetores são representados por letras sublinhadas e matrizes por letras maiúsculas em negrito.

Por simplicidade de notação, algumds gạs letras, mencionadas acima, voltam a se repetir no Capítulo 8. 


\section{Conteúdo}

1 Introdução 1

1.1 Importância das Relações Estresse-Resposta . . . . . . . . . . . 5

1.2 Algumas das Relações Estresse-Resposta mais Utilizadas em Testes de sobrevivência Acelerados ................. 5

1.2.1 O Modelo Lei de Potência Inversa . . . . . . . . . . 6

1.2.2 O Modelo de Taxa de Reação Arrhenius . . . . . . . . . . 7

1.2.3 0 Modelo de Eyring . . . . . . . . . . . . . 7

1.3 Revisão Bibliográfica e Objetivos ... . . . . . . . . . 9

2 Análise Clássica do Modelo de Eyring com uma Variável Estresse 18

2.1 O Experimento em Testes de Sobrevivência Acelerados com Dados Censurados e uma Variável Estresse . . . . . . . . . . . . . 19

2.2 Formulação do Modelo ... . . . . . . . . . . . 20

2.3 Estimadores de Máxima Verossimilhança para os Parâmetros $\alpha$ e $\beta$. 22

2.4 Inferência Sobre os Parâmetros $\alpha$ e $\beta$ ou Funções dos Mes̈mos . . . . 23 
2.5 O Problema da Inferência sobre $\theta_{1}$ Usando a Normalidade Assintótica dos Estimadores de Máxima Verossimilhança . . . . . . . . . 27

2.6 O Estudo de Diferentes Parametrizações $\ldots \ldots \ldots \ldots \ldots \ldots$

2.6.1 Reparametrização Ortogonal: Ortogonalização em $\beta \ldots$. . . 31

2.6.2 Uma Proposta para Obtenção de Estimadores de $\alpha$ e $\beta$ Assintoticamente Independentes . . . . . . . . . . . . 35

2.6.3 Reparametrização Logarítmica em $\theta_{1} \ldots \ldots \ldots \ldots$

2.6.4 Parametrização Assintoticamente Independente . . . . . . 41

2.6.5 Retrospecto das Parametrizações Adotadas . . . . . . . . 46

3 Uma Análise Bayesiana do Modelo de Eyring com uma Variável Estresse

3.1 Densidade a Priori Não-Informativa para $\alpha$ e $\beta \ldots \ldots \ldots \ldots$

3.2 Densidade a Posteriori'Conjunta para $\alpha$ e $\beta \ldots \ldots \ldots \ldots$

3.3 Densidades a Posteriori Marginais para $\alpha$ e $\beta \ldots \ldots \ldots$

3.3.1 Densidade a Posteriori Marginal para $\beta \ldots \ldots \ldots \ldots$

3.3.2 Densidade a Posteriori Marginal para $\alpha \ldots \ldots \ldots \ldots 1$

3.4 Densidade a Posteriori para o Tempo Médio de Sobrevivência, $\theta_{1}$, sob o nivel Usual de Estreșe . . . . . . . . . . . . . . . . 53

3.5 Reparametrização logarítmica em $\theta_{1} \ldots \ldots \ldots \ldots \ldots \ldots$

3.6 O uso de uma Parametrização Independente $\ldots \ldots \ldots \ldots 7$ 
4.1 Densidade Preditiva para uma Observação Futura em um Nível de

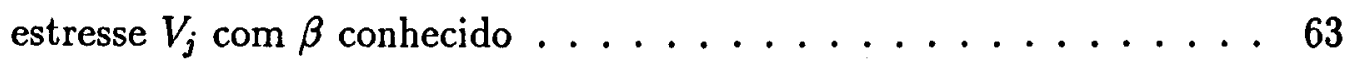

4.2 Densidade Preditiva para uma Observação Futura em um Nível de Estresse $V_{j}$ assumindo $\beta$ desconhecido $\ldots \ldots \ldots 65$

4.3 O Uso da Densidade Preditiva $f^{j}\left(t_{(n+1) j} /\right.$ Dados $)$ em Controle de Qualidade . . . . . . . . . . . . . . . 67

5 Análise do Modelo de Eyring Assumindo uma Distribuição de Weibull para os tempos de Sobrevivência

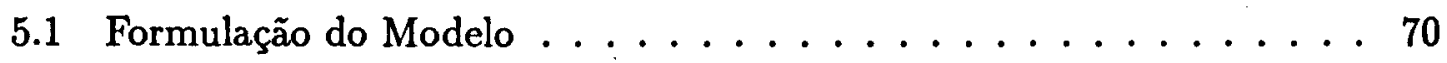

5.2 Análise Bayesiana do Modelo Assumindo $\alpha, \beta$ e $p$ desconhecidos $\ldots 72$

5.2.1 Densidade a Posteriori Marginal para $\mathrm{p} \ldots \ldots \ldots \ldots \ldots 74$

5.2.2 Densidade a Posteriori Conjunta para $\theta_{1}^{*}$ e $p \ldots \ldots \ldots$

6 Testes de Sobrevivência Acelerados com uma Relação EstresseResposta Geral 78

6.1 . A Relação Estresse-Resposta Geral . . . . . . . . . . . 79

6.2 Procedimento Bayesiano de Análise com uma Densidade de Weibull . 80

6.2.1 Densidade a Posteriori Marginal para $p \ldots \ldots \ldots \ldots$. . . 81

6.2.2 Densidade a Posteriori Conjunta para $\theta_{1}^{*}$ e $p \ldots \ldots$. . 82

6.3 Análise Bayesiana assumindo uma Distribuição Exponencial para $T^{*}$. 83 
6.4 A Função de Confiabilidade em Experimentos Acelerados . . . . . 85

6.4.1 Densidade a Posteriori Conjunta para a Função de Confiabilidade $R_{1}^{*}$ e para o Parâmetro de Forma $p$ da Distribuição de Weibull .................. 86

6.4.2 Densidade a Posteriori para $R_{1}^{*}$ Assumindo uma Distribuição Exponencial para $T^{*} \ldots \ldots \ldots \ldots$. . . . . . . 87

7 Experimentos Acelerados com Níveis de Estresse Somente Maiores que os Usuais

7.1 Determinação do Modelo Considerando somente niveis de estresse maiores que os usuais . . . . . . . . . . . . 90

7.2 Uma Análise Bayesiana do Modelo . . . . . . . . . . . . 99 91

7.3 Análise Bayesiana assumindo $p_{2} \doteq p_{3}-1 \ldots \ldots \ldots \ldots$

8 Análise de Testes de Sobrevivência Acelerados com o Modelo de Eyring Generalizado

8.1 Formulação do Experimento Acelerado . . . . . . . . . . 98

8.2 Procedimento usual de Análise do Modelo $\ldots \ldots \ldots \ldots \ldots$

8.3 Análise Bayesiana do Modelo de Eyring Generalizado assumindo uma Distribuição Exponencial para os tempos de Sobrevivência . . . . . 101

8.3.1 Uma Análise Bayesiana assumindo $\alpha, \beta, \gamma$ e $\delta$ desconhecidos .102

8.3.2 Uma Análise Bayesiana assumindo $\gamma$ e $\delta$ conhecidos . . . . . 104

8.3.3 Uma Análise Bayesiana assumindo $\beta, \gamma$ e $\delta$ conhecidos $\ldots 107$ 
8.4 Análise Bayesiana do Modelo de Eyring Generalizado assumindo uma Distribuição de Sobrevivência de Weibull . . . . . . . . . . . . 109

8.4.1 Procedimento Bayesiano de Análise Assumindo todos os Parâmetros Desconhecidos . . . . . . . . . . . . . . . 109

8.4.2 Procedimento Bayesiano de Análise assumindo $\gamma$ e $\delta$ conhecidos 112

9 Exemplos de Aplicação

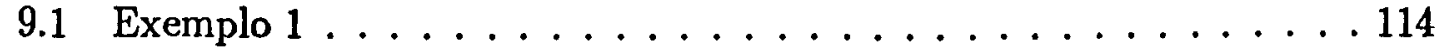

9.1.1 Verificação Gráfica da Adequabilidade do Modelo . . . . . . 116

9.1.2 Análise Clássica do Problema . . . . . . . . . . . 117

9.1.3 Uma Solução Bayesiana para o Problema . . . . . . . . . . 119

9.1.4 Comparação entre ạs Estimativas Pontuais . . . . . . . . 121

9.1.5 Comparação entre as Estimativas por Intervalo . . . . . . 122

9.1.6 O Efeito de Reparametrização na Análise Bayesiana do Modelo125

9.1.7 Controle de Qualidade de um Lote de Componentes . . . . . 127

9.2 Exemplo 2 . . . . . . . . . . . . . . . . . 129

9.3 Exemplo $3 \ldots \ldots \ldots \ldots$

10 Conclusões Finais e Perspectivas Futuras 144

10.1 Perspectivas de Trabalhos Futuros . . . . . . . . . . . . 146 
Apêndice 1: O Método "Delta"

Apêndice 2: O Método de Aproximação de Laplace para Intgerais 154

Apêndice 2.1: Cáculos para obtenção da Densidade a Posteriori Marginal para o



Apêndice 3: Teoria da Verossimilhança: Regiões e Intervalos de Verossimilhança 161

Apêndice 4: Obtenção de Intervalos HPD 165

Bibliografia 167 


\section{Capítulo 1}

\section{Introdução}

O tempo de duração de um determinado produto manufaturado, bem como o seu tempo de garantia, são fatores determinantes na relação produção-consumo, motivações permanentes de pesquisas industriais relacionadas com um controle de qualidade eficiente.

Em engenharia, diz-se que o objetivo é a análise da coṇtıavilidade do componente (produto). Em particular, uma quantidade básica de interesse é o tempo médio de sobrevivência do componente sob condições normais de funcionamento em que usualmente é submetido, isto é, condições que assemelham-se às condições ambientes em que o componente em questão é submetido para operar.

Para estabelecer medidas relacionadas à confiabilidade do componente, torna-se necessário, inicialmente, planejar um experimento considerando uma amostra de componentes, e a resposta de interesse é dada por seus tempos de sobrevívência.

Escolhido um componente de interesse, um experimento trivial consiste em colocarmos $n$ desses componentes em teste, nas condições normais de funcionamento em que usualmente são submetidos, e esperarmos até que todos falhem. Pode-se per- 
ceber que, em muitos casos, dependendo do tipo de componente, como por exemplo, capacitores, transistores, isto é, dispositivos elétricos, eletrônicos, etc., o tempo e, consequentemente, o custo de experimentação, podem se tornar demasiadamente grandes - praticamente inviável para indústrias de varias áreas. Uma alternativa é diminuir artificialmente o tempo de sobrevivência dos componentes através dos chamados Testes de Sobrevivência Acelerados, nos quais, considera-se vários níveis de uma ou mais variáveis físicas que estressam o componente acima dos níveis usuais de funcionamento (condições normais de funcionamento). Dessa forma, temos possibilidade de observar o tempo de sobrevivência, com tempo e custo reduzidos.

Aplicações de condições de aceleração, que induzem à redução do tempo de sobrevivência, em geral, em engenharia, são produzidas por testes de componentes, sub-sistemas ou sistemas, em temperatura, tensão elétrica, pressão, umidade, etc., maiores que as comumente utilizadas. Com menor frequência, essas condições podem ser observadas em experimentações biológicas, que aparecem quando da aplicação de doses maiores do que as usuais de’agentes químicos-farmacológicos e/ou radiobiológicos. Em ambos os casos, os dados obtidos sob condições aceleradas são utilizados para explorarmos algum nivel baixo de estresse, onde o teste, em geral, é inviável dado o grande aumento do tempo e do custo de experimentação. Particularmente, em engenharia, esses dados são utilizados para inferirmos sobre o tempo médio de sobrevivência do componente, nas condições normais de funcionamento; nas áreas biológicas, para inferências sobre o tempo médio de sobrevivência de indivíduos (animais, vegetais) nas doses usuais dos agentes causadores de transformações patológicas.

Formalmente, seja $T$ uma variável aleatória não-negativa, denotando o tempo de sobrevivência de um componente, com função densidade de probabilidade (f.d.p.) dada por, $f(t, \underline{\lambda})$, onde $\underline{\lambda}$ é um vetor de parâmetros, e seja $\underline{S}$ um vetor de variáveis físicas que estressam o componente com niveis maiores do que os usuais de 
funcionamento.

Devemos supor que (ver por exemplo, Mann, Schaffer e Singpurwalla, 1974):

a) o tipo da distribuição do tempo de sobrevivência não é afetado pelas condições de aceleração, caracterizadas em $S$, mas os níveis de estresse influenciam os valores dos parâmtros em $\underline{\lambda}$;

b) a relação entre $\lambda$ e $S$ (relação estresse-resposta) é conhecida, isto, é $\Delta=$ $g(\underline{S}, \alpha, \beta, \ldots)$, exceto para um ou mais dos parâmetros $\alpha, \beta, \ldots, \mathrm{e}$ é válida para um determinado intervalo de variação das variáveis em $\underline{S}$.

O principal objetivo do pesquisador, nesta área, é fazer inferências sobre $\underline{\lambda}$ ou funções de $\underline{\lambda}$, baseado em dados sobre $T$ obtidos sob condições aceleradas.

Nos testes de sobrevivência acelerados, considera-se th niveis aleatorizados de uma ou mais variáveis estresse em $\underline{S}$, aplicados ao componente. Em cada nível $i$ de estresse, $i=2,3, \ldots, k$, uma ou mais unidades são submetidas a teste. $O$ nível $\mathrm{i}$ $=1$ é reservado às condições usuais de funcionamento.

Supondo que $n_{i}$ unidades são colocadas em teste no i-ésimo nível de estresse e que $m$ variáveis físicas de estresse estão envolvidas no processo, isto é, $\underline{S}^{\prime}=$ $\left(S_{1}, S_{2}, \ldots, S_{m}\right)$, construímos a Tabela 1.1 do esquema de um experimento em testes de sobrevivência acelerados, onde, $S_{2}<S_{3}<\ldots<S_{k \text {. }}$, em cada uma das $m$ variáveis.

Considerando a relação estresse-resposta, $g($.), e assumindo a distribuição $f(t, \underline{\lambda})$ para os tempos de sobrevivência, inferências sobre o tempo médio de sobrevivência, $\theta_{i}$, sob o i-ésimo nivel de estresse, podem ser obtidas. Particularmente, pode-se inferir sobre o tempo médio de sobrevivência, $\theta_{1}$, sob os níveis usuais de 


\begin{tabular}{cccc}
$i$ & $S_{1}, S_{2}, \ldots, S_{m}$ & $n_{i}$ & Dados observados \\
\hline \hline 2 & $S_{21}, S_{22}, \ldots, S_{2 m}$ & $n_{2}$ & $t_{21}, t_{22}, \ldots, t_{2 n_{2}}$ \\
3 & $S_{31}, S_{32}, \ldots, S_{3 m}$ & $n_{3}$ & $t_{31}, t_{32}, \ldots, t_{3 n_{3}}$ \\
$\vdots$ & $\vdots$ & $\vdots$ & $\vdots$ \\
$k$ & $S_{k 1}, S_{k 2}, \ldots, S_{k m}$ & $n_{k 1}$ & $t_{k 1}, t_{k 2}, \ldots, t_{k n_{k}}$ \\
\hline
\end{tabular}

Tabela 1.1: Esquema de um experimento em testes de sobrevivência acelerados. estresse, $S_{11}, S_{12}, \ldots, S_{1 m}$, como visto anteriormente, quantidade básica de interesse na análise da confiabilidade de componentes manufaturados.

Se o objetivo é reduzir ainda mais o tempo e o custo do experimento, podese associar os experimentos sob condições aceleradas a dados sob algum esquema de censura (ver por exemplo, Lawless, 1982).

A associação de algum esquema de censura aos experimentos acelerados torna-se, em geral, necessária, quanolo consideramos níveis baixos de estresse no experimento, onde, como visto anteriormente, o teste pode se tornar inviável.

Dessa forma, pode-se óbservar um número grande de censuras nesses níveis de estresse, garantindo a viabilidade do teste, conservando o tempo e, consequentemente, o custo do experimento, reduzidos. Neste contexto, como em geral, o interesse é explorar o nível usual de estresse, pode-se, quando possível, conduzir um teste acelerado, considerando-se $n_{1}$ componentes submetidos aos níveis usuais, $S_{11}, S_{12}, \ldots, S_{1 m}$, das variáveis estresse, associados a algum esquema de censura, neste caso, $i=1,2, \ldots, k$. 


\subsection{Importância das Relações Estresse-Resposta}

Basicamente, a distribuição de sobrevivência de um determinado componente expressa uma tentativa de descrever matematicamente sua duração . Paralelamente, muitas variáveis físicas podem ser responsáveis, individual e/ou coletivamente, por sua falha, em um determinado instante. Em geral, considerar todas as variáveis físicas, possíveis causadoras da falha, torna-se inviável. Daí, a utilização de relações estresse-resposta, obtidas dos conceitos da mecânica estatística (ver por exemplo, Mann Schaffer e Singpurwalla, 1974), que objetivam isolar somente as variáveis físicas intrinsicamente ligadas ao tempo de sobrevivência do componente (responsáveis pelo mecanismo básico de falha), desconsiderando, com respaldo teórico, outras quaisquer variáveis, passíveis de controle experimental e/ou que não influenciam diretamente o tempo de sobrevivência.

\subsection{Algumas das Relações Estresse-Resposta mais Utilizadas em Testes de sobrevivência Ace- lerados}

Considere a variável aleatória $T$, definida anteriormente, com f.d.p. $f(t, \lambda)$. Em particular, seja $T \geq 0$ com uma distribuição Exponencial, com f.d.p. dada por,

$$
f\left(t, \lambda_{i}\right)=\lambda_{i} \exp \left\{-\lambda_{i} t\right\}
$$

onde $\lambda_{i}>0\left(\lambda_{i} \in \underline{\lambda}\right)$ é um parâmetro desconhecido representando a taxa constante de falha em um determinado nível $i$ de estresse.

O tempo médio de sobrevivência, $\theta_{i}$ (função de $\underline{\lambda}$ ), sob o i-ésimo nível de 
estresse é dado por,

$$
\theta_{i}=\frac{1}{\lambda_{i}}
$$

De fato, além de depender dos tempos de sobrevivência dos componentes, $\theta_{i}$ também depende das condições de aceleração através de $\lambda_{i}$, que podem ser expressas pela relação estresse-resposta $g($.$) , isto é, além de uma distribuição para os tempos$ de sobrevivência, associamos ao tempo médio de sobrevivência, $\theta_{i}$, uma ou mais variáveis físicas de estresse que influenciam seu valor.

Das relações estresse-resposta mais conhecidas, mencionadas na literatura e utilizadas na prática, destacam-se o modelo Lei de Potência Inversa, 'o modelo de Taxa de Reação de Arrhenius, e o modelo de Eyring.

A construção desses modelos respalda-se, como visto anteriormente, nos conceitos da mecânica estatística, tais como energia de ativação e teorias de taxa de reação (ver por exemplo, Mann, Schaffer e Singpurwalla, 1974).

\subsubsection{O Modelo Lei de Potência Inversa}

O Modelo Lei de Potência Inversa, também conhecido como modelo Potência, determinado a partir de considerações da teoria cinética e energia de ativação , é dado por,

$$
\lambda_{i}^{-1}=\frac{\alpha}{V_{i}^{\beta}},
$$

onde $V_{i}$ é o i-ésimo nívél da variável estresse de voltagem, $V$, e $\alpha$ e $\beta$ são parâmetros tais que, $\alpha>0$ e $-\infty<\beta<\infty$. 
Esse modelo expressa o aumento da taxa de falha $\lambda$ com a $\beta$-ésima potência da voltagem $V$ aplicada ao componente e tem como uma de suas aplicaçôes o teste, sob condições aceleradas, de capacitores (ver por exemplo, Lavenbach, 1957).

\subsubsection{O Modelo de Taxa de Reação Arrhenius}

Representando a variação da taxa de falha como uma função da temperatura de operação , o modelo de Taxa de Reação de Arrhenius, também chamado modelo de Arrhenius, é dado por,

$$
\lambda_{i}=\exp \left\{\alpha-\frac{\beta}{V_{i}}\right\}
$$

onde $V_{i}$ é o i-ésimo nivel da variável estresse de temperatura, $V$, e $\alpha$ e $\beta$ são os parâmetros desconhecidos, $-\infty<\alpha, \beta<\infty$.

Aplicações deste modelo em testes de sobrevivência acelerados poderh ģen vistas em Thomas (1964).

\subsubsection{O Modelo de Eyring}

O Modelo de Eyring pode ser descrito em uma versão simplificada, envolvendo somente uma variável estresse de temperatura, e em uma versão generalizada, onde a taxa de falha, $\lambda$, é expressa em função de duas variáveis estresse, uma termal e outra não-termal.

\section{O Modelo de Eyring com uma Variável Estresse}

Derivado dos princípios da mecânica quântica, o modelo de Eyring com uma variável estresse expressa a variação da taxa de falha como uma função da 
temperatura de operação (ver por exemplo, McGraw-Hill Encyclopedia of Science Techology, vol. 7, pg. 357), e é dado por,

$$
\lambda_{i}=V_{i} \exp \left\{\alpha-\frac{\beta}{V_{i}}\right\}
$$

onde $V_{i}$ é o i-ésimo nivel da variável estresse de temperatura, $\mathrm{V}$, e $\alpha$ e $\beta$ são parâmetros desconhecidos, $-\infty<\alpha, \beta<\infty$.

\section{O modelo de Eyring Generalizado}

Em sua versão generalizada, esse modelo é aplicável se o componente, em questão está sujeito à aplicação constante de dois tipos de estresse, um termal e outro não-termal (ver por exemplo, Mann, Schaffer e Singpurwalla, 1974), e é dado por,

$$
\lambda_{i}=\alpha V_{i} \exp \left\{\gamma U_{i}+\delta \frac{U_{i}}{k_{1} V_{i}}-\beta \frac{1}{k_{1} V_{i}}\right\}
$$

onde $V_{i}$ é o i-ésimo nível da variável estresse de temperatura, $U_{i}$ é o i-ésimo nível da variável estresse não-termal, $k_{1}$ é a constante universal de Boltzmann $\left(k_{1}=\right.$ $\left.1,38.10^{-16} \mathrm{erg} / k^{\circ}\right)$ e $\alpha, \beta, \gamma$ e $\delta$ são parâmetros desconhecidos tais que, $\alpha>0 \mathrm{e}$ $-\infty<\beta, \gamma, \delta<\infty$.

Uma aplicação desse modelo em teste de sobrevivência acelerado de películas de filme é discutida por Goldberg (1964).

Deve-se enfatizar que as relações estresse-resposta (modelos) descritas acima podem ser válidas somente dentro de um determinado intervalo de variação das variáveis estresse envolvidas, e para serem extendidas a valores fora dos limites do 
mesmo deve-se ter cautela. Isto devido ao fato de uma possivel mudança no mecanismo básico de falha, responsável pela validade de um determinado modelo, $g($.$) .$ Quando necessário, deve-se formular outra relação estresse-resposta que expresse o tempo médio de sobrevivência em função dos novos valores (fora dos limites do intervalo de variação previamente determinado) das variáveis estresse em questão (ver por exemplo, Mann, Schaffer e Singpurwalla, 1974).

Além disso, a estrutura probabilística de $T, f(t, \lambda)$, pode ser dada por outras distribuições, além da Exponencial, considerada até o momento, como por exemplo, a Distribuição de Weibull, a Log-Normal, a Gamma, etc.

\subsection{Revisão Bibliográfica e Objetivos}

O problema de analisar testes de sobrevivência acelerados, considerando somente uma relação estresse-reposta, tem sido tema de pesquisa para vários autores.

Uma extensa bibliografia de aplicações desses testes pode ser encontrada em Nelson (1974).

Quando a distribuição dos tempos de sobrevivência dos componentes é Exponencial e os dados são não censurados (completos), Nelson (1972c) descreve métodos gráficos para a estimação dos parâmetros do modelo de Lei de Potência Inversa. Com dados sob o esquema de censura de tipo II (ver por exemplo, Lawless, 1982), Mann, Schaffer e Singpurwalla (1974) determinam os estimadores de máxima verossimilhança para os parâmetros dos modelos Potência, Arrhenius e Eyring com uma variável estresse, obtendo estimadores assintoticamente independentes, e um estimador não-viciado para o tempo médio de sobrevivência, $\theta_{i}$, sob o i-ésimo nível de estresse. Também, sugerem a obtenção dos limites de confiança para esses parâmetros via função de vërossimilhança relativa maximizada (ver por 
exemplo, Kalbfleisch, 1985). Além disso, determinam os estimadores de mínimos quadrados para os parâmetros do modelo de Eyring generalizado e discutem estratégias de planejamento de experimentos acelerados.

Assụmindo uma relação estresse-resposta que generaliza os modelos Potência e Arrhenius, uma distribuição Exponencial, e dados sob esquema de censura de tipo II, Yum e Kim (1990) apresentam um procedimento de planejamento amostral, através do qual determinam o número de falhas requerido em um determinado nível de estresse que será usado em um experimento acelerado com dois niveis de estresse maiores que o usual.

Com dados sob esquema de censura de tipo I ou II (ver por exemplo, Lawless, 1982), e uma relação estresse-resposta que generaliza os modelos mais utilizados na prática que consideram uma única variável estresse, Barbosa e Louzada Neto (1991a) obtém uma análise geral para testes acelerados via Modelos Lineares Generalizados (ver por exemplo, McCullagh e Nelder, 1989).

Louzada Neto e Rodrigues (1991) estudam o efeito da reparametrização ortogonal (ver por exemplo, Cox e Reid, 1987) na inferência sobre o tempo médio de sobrevivência, $\theta_{1}$, de um determinado componente, sob o nível usual de estresse, assumindo uma distribuição Exponencial, dados sob esquema de censura de tipo II e quando a relação estresse-resposta é representada pelo modelo Potência; e mostram que a função de verossimilhança maximizada para esse parâmetro é invariante sobre esse tipo de reparametrização :

Uma solução Bayesiana para o problema de estimação dos parâmetros do modelo Potência, assumindo uma distribuição Exponencial para os tempos de sobrevivência e dados sob esquema de censura de tipo II, pode ser encontrada em Achcar e Dias (1990b) que obtém a densidade a posteriori marginal para o tempo médio de sobrevivência, $\theta_{1}$, e apresentam algumas sugestões sobre a determinação do número 
de falhas requerido em um experimento acelerado.

Em Rodrigues, Achcar e Louzada Neto (1990) encontramos uma expressão em "forma fechada" para a moda da densidade a posteriori de $\theta_{1}$, obtida via reparametrização Ortogonal, considerando o modelo de Potência.

Achcar (1990a) desenvolve um procedimento de controle de qualidade usando densidades preditivas (ver por exemplo, Aichinson e Dunsmore, 1975) quando o modelo de Potência é adequado aos dados, assumindo uma distribuição Exponencial. Através do procedimento obtido, considerando um determinado nível de estresse fixo, obtém o tempo requerido de experimentação em um teste acelerado, e também, o nível de estresse, sob o qual o teste deve ser feito, considerando o tempo de experimentação fixo.

Considerando uma reparametrização ortogonal, Rodrigues (1991) obtém a densidade preditiva de uma observação futura sob o nível usual de estresse.

Vários autores tem abordado em suas pesquisas testes de sobrevivêntia acelerados, considerando outras distribuições para os tempos de sobrevivência.

Entre outros, considerando uma distribuição de Weibull, podemos citar Nelson (1970) que encontra os estimadores de mínimos quadrados e de máxima verossimilhança para os parâmetros do modelo Potência quando os dados são completos; Mann (1972) que discute uma estratégia de planejamento para esses testes assumindo que o logarítmo do parâmetro de escala da distribuição de Weibull é uma função polinomial de grau conhecido do inverso do nível de estresse, e que o parâmetro de forma não depende desse nível. Pelo planejamento obtido, especifica o número e quais níveis de estresse devem ser usados no teste, também, qual a proporção de elementos da amostra total que deve ser alocada aleatoriamente em cada nível de estresse. 
Simulando experimentos fatoriais como número de níveis de estresse, porcentagens de censura e tamanhos de amostras diferentes, Bugaighis (1988) compara os estimadores de máxima verossimilhança com os melhores estimadores lineares não-viciados dos modelos de Potência e Arrhenius, quando a distribuição de sobrevivência é Weibull, de acordo com o critério do erro médio quadrático, e observa que, em geral, os dois tipos de estimadores tem eficiência equivalente, com excessão nas situações onde o número de censuras é muito grande, deixando algum nível da variável estresse com duas ou menos observações. Nestas situações, os estimadores de máxima verossimilhança são claramente melhores que os melhores estimadores lineares não-viciados.

Considerando também os modelos de Potência e Arrhenius e uma distribuição de Weibull, Wiell e Meeker (1990) mostram, através de simulação, que em um teste de sobrevivência acelerado, intervalos de confiança obtidos via teste da razão de verossimilhança são melhores que os intervalos de confiança baseados na teoria Normal assintótica.

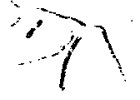

Barbosa e Louzada Neto (1991b), utilizam Modelos Lineares Generalizados e técnicas de inferência relácionadas e determinam inferências pontuais e por intervalo para o tempo médio de sobrevivência de uma unidade sob a presença de dados censurados, considerando uma distribuição de sobrevivência de Weibull e uma relação estresse-resposta geral que tem como casos particulares os modelos Potência, Arrhenius, Eyring com uma variável estresse e Eyring generalizado.

Assumindo uma distribuição Normal ou Log-Normal para os tempos de sobrevivência, em uma série de artigos, Nelson (1971, 1972a, 1972b) determina os estimadores gráficos, de mínimos quadrados e de máxima verossimilhança para os parâmetros do modelo de Arrhenius quando todos os tempos de falha são conhecidos, assumindo que a média do logarítimo do tempo de falha é linear na variável estresse e 
que a variância é independente desta variável. Também discute a utilização de alguns critérios relacionados a planejamento de experimentos acelerados, em particular, a minimização da variância do estimador do tempo médio de sobrevivência sob o nível usual de estresse.

Achcar e Rosales (1991c) obtém uma análise Bayesiana para testes acelerados considerando uma relação estresse-resposta linear no inverso da média da distribuição Gaussiana Inversa. Também propõe alguns critérios para serem usados em problemas de controle de qualidade.

Existem várias situações onde mais que uma relação estresse-resposta é considerada. Nestes casos, o tempo de sobrevivência de um determinado componente depende de uma causa de falha entre outras causas importantes. Entre outros, Klein e Basu (1981) descrevem uma análise de testes de sobrevivência acelerados de sistemas em série (ver por exemplo, Bain, 1978), assumindo uma distribuição de Weibull para os tempos de sobrevivência dos componentes com parâmetro de forma diferentel para cada um dos componentes do sistema, dados sob o esquema de censura de tipo I, II ou progressiva (ver por exemplo, Lawless, 1982), e um modelo geral que inclui os modelos-padrốes: Potência, Arrhenius e Eyring com uma variável estresse.

Vários autores tem analisado testes de sobrevivência acelerados no enfoque não-paramétrico. Entre outros, podemos citar, Shaked, Zinner e Ball (1979), Shaked e Singpurwalla (1982), Basu e Ebrahimi (1982).

Em muitas situações, dispomos somente de uma unidade para ser testada. Também, o componente manufaturado é feito para suportar um conhecido e especificado nível de estresse. Supondo que no teste verifica-se somente a sobrevivệncia ou falha da unidade em um determinado nível de estresse, Martz e Waterman (1978) desenvolvem um procedimento Bayesiano para determinar o nivel de estresse reque- 
rido em um teste acelerado que maximiza a probabilidade esperada do componente sobreviver no nível de estresse conhecido.

Até o momento, uma suposição básica é termos distribuição de sobrevivência com mesma forma em todos os níveis de estresse. Porém, em muitas aplicações práticas, esta suposição pode não ser apropriada, e isto implica que os diferentes niveis de estresse podem ter diferentes efeitos no mecanismo causador das falhas, e do ponto de vista físico, isso pode ser mais realista, além de permitir diferentes formas de distribuição de sobrevivência nos diferentes níveis de estresse. Neste contexto, Proschan e Singpurwalla (1979), não supõe distribuição de sobrevivência alguma, nem relação estresse-resposta, e propõe um modelo não-paramétrico para testes de sobrevivência acelerados, utilizando inferência Bayesiana.

Finalmente, Nelson (1990), por ser o primeiro livro inteiramente voltado para testes de sobrevivência acelerados, é uma bibliografia indispensável, onde encontramos vários tópicos relacionados a esse tema. Entre outros, modelos para testes de sobrevivência com estresse constante, análises gráficas, de mínimos quadrados e de máxima verossimilhança, planejamento de experimentos acelerados, modelos de sobrevivência com riscos competitivos, etc.

Nesta dissertação, consideramos as distribuições Exponencial e de Weibull para os tempos de sobrevivência dos componentes, e dados sob esquema de censura tipo II.

Apresentamos, no Capítulo 2, uma análise clássica do problema de inferência em testes de sobrevivência acelerados considerando o modelo de Eyring com uma variável estresse (1.5) e uma distribuição de sobrevivência Exponencial, e discutimos a ineficiência dos métodos tradicionais de estimação por intervalo baseados na teoria Normal assintótica. Consideramos também, neste capítulo, o estudo de algumas reparametrizações dos parâmetros envolvidos com a finalidade de obtermos 
melhor aproximação Normal assintótica para suas funções de verossimilhança, como por exemplo, a reparametrização ortogonal (ver por exemplo, Cox e Reid, 1987) e a logarítmica.

Considerando densidades a priori não-informativas de Jeffreys (ver por exemplo, Box e Tiao, 1973) para os parâmetros envolvidos, determinamos, no Capítulo 3, uma análise Bayesiana do Modelo de Eyring com uma variável estresse (1.5), explorando o uso do método de Laplace para aproximação de integrais (ver por exemplo, Tierney e Kadane, 1986; Kass, Tierney e Kadane, 1990), quando não conseguimos as soluções analíticas explícitas para as densidades a posteriori de interesse. A precisão dos valores aproximados também é estudada.

Transformações de variáveis são adotadas com o intuito de obtermos densidades a priori não-informativas de Jefreys localmente uniforme, que implicam, em geral, em uma boa precisão nas aproximações das densidades a posteriori marginais via método de Laplace.

Ainda considerando o modelo de Eyring com uma varável estresse (1.5) e uma distribuição Exṕonencial para os tempos de sobrevivência, desenvolvemos no Capítulo 4 um procedimento de controle de qualidade de um lote de componentes, utilizando a densidade preditiva de uma observação futura (ver por exemplo, Aitchinson e Dunsmore, 1975), em um nível especificado de estresse.

No Capítulo 5 obtemos uma análise Bayesiana do modelo de Eyring com uma variável estresse (1.5) assumindo uma distribuição de sobrevivência de Weibull com parâmetro de forma comum em todos os níveis de estresse. A regra multiparamétrica de Jeffreys (ver por exemplo, Box e Tiao, 1973) é usada para acharmos uma densidade a priori não-informativa para os parâmetros envolvidos.

Definindo uma relação estresse-resposta geral com uma única variável estresse que inclui , como casos particulares, os modelos Potência (1.3), Arrhenius (1.4) 
e Eyring com uma variável estresse (1.5), determinamos, no Capítulo 6, um procedimento Bayesiano de análise válido para os três modelos em questão, assumindo uma distribuição de Weibull para os tempos de sobrevivência dos componentes, com parâmetros de forma comum para todos os níveis de estresse.

Em todos os capítulos mencionados, sempre consideramos o nível usuạl da variável estresse no teste acelerado. No Capítulo 7, essa limitação é suprimida, e somente com dois niveis de estresse estritamente maiores que os usuais e uma distribuição de sobrevivência de Weibull com parâmetro de forma diferente em cada um desses dois niveis, determinamos uma análise Bayesiana da relação estresseresposta geral definida no capitulo anterior.

No Capítulo 8, inicialmente assumindo uma distribuição Exponencial para os tempos de sobrevivência, desenvolvemos um procedimento Bayesiano de análise do modelo de Eyring generalizado (1.6). Também, obtemos uma análise Bayesiana considerando esse modelo com dois parâmetros conhecidos e uma distribuição de sobrevivência de Weibull, mostrando sua equivalência com 6 análise da relação estresse-resposta descrita no Capítulo 6.

Exemplos de aplicação das metodologias obtidas são desenvolvidos no Capítulo 9.

Finalmente no Capítulo 10, são apresentadas algumas conclusões e comentários sobre as perspectivas futuras de pesquisas vinculadas a testes de sobrevivência acelerados.

Devemos informar que as análises obtidas nos Capítulos de 2 a 4 podèm ser encontradas em Achcar e Louzada Neto (1991a). Em Achcar e Louzada Neto (1991b) encontramos a metodologia de análise que está no Capítulo 6, e em Achcar e Louzada Neto (1990c) temos os procedimentos Bayesianos determinados no Capítulo 8, assumindo-se uma distribuição Exponencial para os tempos de sobrevivência dos 
componentes.

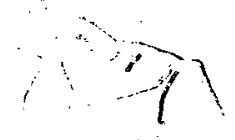




\section{Capítulo 2}

\section{Análise Clássica do Modelo de Eyring com uma Variável Estresse}

O modelo de Eyring com uma variável estresse (1.5), como visto no capítulo anterior, encontra-se entre as relações estresse-resposta mais utilizadas na prática para descrever a taxa de falha de um determinado componente manufaturado em função de uma variável estresse de temperatura. Além disso, apesar de intuitivamente observarmos que as taxas de falha crescem com o uso (envelhecimento) para a maioria dos componentes, podemos assumir, em um estudo inicial, que as falhas. ocorrem aleatoriamente e considerarmos a distribuição Exponencial (amplamente utilizada em confiabilidade) para expressar matematicamente o comportamento dos tempos de sobrevivência.

Neste capítulo, considerando uma distribuição Exponencial para os tempos de sobrevivência e dados sob esquema de censura tipo II (ver por exemplo, Lawless, 1982), determinamos os estimadores de máxima verossimilhança dos parâmetros do modelo de Eyring com uma variável estresse (1.5), e a partir da aproximação Normal assintótica dos estimadores de máxima verossimilhança, estudamos a precisão das inferências sobre o tempo médio de sobrevivência, $\theta_{1}$, sob os níveis usuais de estresse. Para obtermos melhores aproximações Normais para a função de verossimilhança 
dos parâmetros envolvidos, exploramos o uso de reparametrizações.

\subsection{O Experimento em Testes de Sobrevivência Acelerados com Dados Censurados e uma Variável Estresse}

Conforme vimos no capítulo anterior, em confiabilidade, é fundamental que os experimentos sejam eficientes em relação ao seu tempo de duração e consequentemente ao seu custo. Colocando-se unidades para serem testadas sob condições açeleradas de estresse essas exigências são de certa forma satisfeitas. Além disso, podemos considerar dados sob algum esquema de censura, o que reduz ainda mais o tempo e o custo de experimentação, isto é, o experimento termina num tempo $T_{0}$, quando observamos uma dada proporção $q$ de falhas das unidades em testes, num dado nivel $i$ de estresse.

Formalmente, considerando $k$ niveis aleatorizados de uma variável estresse $V \in \underline{S}$, incluindo o nível usual de funcionamento, colocamos $n_{i}$ unidades em teste no, i-ésimo nivel $V_{i}, i=1,2, \ldots, k$, e'término do experimento ocorre quando uma quantidade pré-fixada $r_{i}\left(<n_{i}\right)$ das unidades falham, o que caracteriza dados sob um esquema de censura tipo II (ver por exemplo, Lawless, 1982). Dessa forma, denotando por $T$ o tempo de sobrevivência de uma unidade em teste, temos $t_{i 1}, t_{i 2}, \ldots, t_{i r_{i}}$ tempos observados ordenados (observações não censuradas) dentre as $n_{\boldsymbol{i}}$ unidades no i-ésimo nível de estresse, e $n_{i}-r_{i}$ observações censuradas iguais a $t_{i r_{i}}$, como podemos observar na Tabela 2.1, onde $V_{1}$ é o nível usual de funcionamento do componente. 


\begin{tabular}{ccccc}
$\mathrm{i}$ & $V_{i}$ & $n_{i}$ & $r_{i}$ & DADOS NÃO CENSURADOS \\
\hline \hline 1 & $V_{1}^{(*)}$ & $n_{1}$ & $r_{1}$ & $t_{11}^{(* *)}, t_{12}^{(* *)}, \ldots, t_{1 r_{1}}^{(* *)}$ \\
2 & $V_{2}$ & $n_{2}$ & $r_{2}$ & $t_{21}, t_{22}, \ldots, t_{2 r_{2}}$ \\
$\vdots$ & $\vdots$ & $\vdots$ & $\vdots$ & $\vdots$ \\
$\mathrm{k}$ & $V_{k}$ & $n_{k}$ & $r_{k}$ & $t_{k 1}, t_{k 2}, \ldots, t_{k r_{k}}$ \\
\hline
\end{tabular}

(i) Nivel usual da varibuel floica de estresse.

(**) Tempos de sobrevivencia dos $n_{1}$ componentes em teste sob o nivel usual de estresse da varisvel $v$.

Tabela 2.1: Esquema de um experimento utilizado em testes de sobrevivência acelerados com uma variável estresse e dados com censuras de tipo II.

\subsection{Formulação do Modelo}

A modelagem descrita nesta seção será definida a partir da relação estresse -resposta representada pelo modelo de Eyring com uma variável estresse (1.5) e a distribuição Exponencial para os tempos de sobrevivência dos componentes, considerando o experimento acelerado formulado na seção anterior (ver Tabela 2.1).

Seja $T$ uma variável aleatória não-negativa denotando o tempo de sobrevivência de um componente com uma distribuição Exponencial com função densidade de probabilidade (f.d.p.) dada por,

$$
f\left(t, \lambda_{i}\right)=\lambda_{i} \exp \left\{-\lambda_{i} t\right\}
$$

onde $\lambda_{i}>0$ é um parâmetro desconhecido representando a taxa constante de falha no i-ésimo nível de estresse $V_{i}, i=1,2, \ldots, k$.

O tempo médio de sobrevivência sob o nível de estresse $V_{i}$ é dado por, 


$$
\theta_{i}=1 / \lambda_{i}
$$

obtido calculando-se a esperança do tempo de sobrevivência, $E(T)$.

Assumindo um esquema de censura de tipo II para os dados (ver por exemplo, Lawless, 1982), a função de verossimilhança para $\lambda_{i}$, sob o i-ésimo nível de estresse $V_{i}$, é dada por,

$$
L_{i}\left(\lambda_{i}\right)=\prod_{j=1}^{r_{i}} f\left(t_{i j}, \lambda_{i}\right) S^{n_{i}-r_{i}}\left(t_{i r_{i}}, \lambda_{i}\right)
$$

onde $f\left(t_{i j}, \lambda_{i}\right)$ é a f.d.p. (2.1) que representa a contribuição de uma observação não censurada à função de verossimilhança, e $S\left(t_{i r_{i}}, \lambda_{i}\right)=P\left(T \geq t_{i r_{i}}\right)=\int_{t_{i r_{i}}}^{\infty} \lambda_{i} \exp \left\{-\lambda_{i} t\right\}$ $d t=\exp \left\{-\lambda_{i} t_{i r_{i}}\right\}$ é a função de confiabilidade que expressa a contribuição de um componente (observação) censurado.

Desenvolvendo (2.3) obtemos,

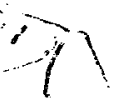

$$
L_{i}\left(\lambda_{i}\right)=\left[\prod_{j=1}^{r_{i}} \lambda_{i} e^{-\lambda_{i} t_{i r_{i}}}\right]\left[e^{-\lambda_{i} t_{i r_{i}}^{\prime}}\right]^{n_{i}-r_{i}}=\lambda_{i}^{r_{i}} \exp \left\{-\lambda_{i}\left[\sum_{j=1}^{r_{i}} t_{i j}+\left(n_{i}-r_{i}\right) t_{i r_{i}}\right]\right\}
$$

Definindo $A_{i}=\sum_{j=1}^{r_{i}} t_{i j}+\left(n_{i}-r_{i}\right) t_{i r_{i}}$, temos:

$$
L_{i}\left(\lambda_{i}\right)=\lambda_{i}^{r_{i}} \exp \left\{-\lambda_{i} A_{i}\right\}
$$

Com dados sob $\mathrm{k}$ níveis aleatorizados de estresse, $V_{1}, V_{2}, \ldots, V_{k}$, a função de verossimilhaça para $\underline{\lambda}^{\prime}=\left(\lambda_{1}, \lambda_{2}, \ldots, \lambda_{k}\right)$ é dada por,

$$
L(\underline{\lambda})=\prod_{i=1}^{k}\left\{\lambda_{i}^{r_{i}} \exp \left[-\lambda_{i} A_{i}\right]\right\}
$$


Considere o modelo de Eyring (1.5) onde a taxa de falha $\lambda$ relaciona-se com o i-ésimo nível da variável estresse $V$ através da relação estresse-resposta,

$$
\lambda_{i}=V_{i} \exp \left\{\alpha-\frac{\beta}{V_{i}}\right\}
$$

Substituindo (2.6) em (2.5) obtemos a função de verossimilhança para $\alpha \mathrm{e}$ $\beta$, dada por,

$$
L(\alpha, \beta)=\prod_{i=1}^{k}\left\{V_{i} \exp \left[\alpha-\beta / V_{i}\right]\right\}^{r_{i}} \cdot \exp \left\{-V_{i} A_{i} e^{\alpha-\beta / V_{i}}\right\}
$$

Dai temos,

$$
L(\alpha, \beta) \propto \exp \left\{\alpha r-\beta a_{1}-e^{\alpha} \sum_{i=1}^{k} V_{i} A_{i} e^{-\beta / V_{i}}\right\},
$$

onde $r=\sum_{i=1}^{k} r_{i}$ é onúmero total de falhas observadas e $a_{1}=\sum_{i=1}^{k} \frac{r_{i}}{v_{i}}$.

\subsection{Estimadores de Máxima Verossimilhança pa- ra os Parâmetros $\alpha$ e $\beta$}

A partir do momento em que observamos os dados - no nosso caso, tempos de sobrevivência dos componentes em teste - toda a informação sobre os parâmetros está contida na função log-verossimilhança.

O logarítmo da função de verossimilhança para $\alpha$ e $\beta$ é dado por,

$$
\ell(\alpha, \beta) \propto \alpha r-\beta a_{1}-e^{\alpha} \sum_{i=1}^{k} V_{i} A_{i} e^{-\beta / V_{i}}
$$

As primeiras derivadas de $\ell(\alpha, \beta)$ com respeito a $\alpha$ e $\beta$ são dadas por, : 


$$
\begin{aligned}
& \frac{\partial \ell(\alpha, \beta)}{\partial \alpha}=r-e^{\alpha} \sum_{i=1}^{k} V_{i} A_{i} e^{-\beta / V_{i}} \\
& \frac{\partial \ell(\alpha, \beta)}{\partial \beta}=-a_{1}+e^{\alpha} \sum_{i=1}^{k} A_{i} e^{-\beta / V_{i}} .
\end{aligned}
$$

Igualando a zero as primeiras derivadas (2.9) e (2.10), e resolvendo o sistema de equações em $\alpha$ e $\beta$, obtemos os estimadores de máxima verossimilhança de $\alpha$ e $\beta$ dados por,

$$
\hat{\alpha}=\log \left[\frac{r}{\sum_{i=1}^{k} V_{i} A_{i} e^{-\hat{\beta} / V_{i}}}\right]
$$

e $\hat{\beta}$ que é a solução da equação

$$
\frac{\sum_{i=1}^{k} A_{i} e^{-\hat{\beta} / V_{i}}}{\sum_{i=1}^{k} V_{i} A_{i} e^{-\hat{\beta} / V_{i}}}=\frac{a_{1}}{r}
$$

Como as equações (2.11) e (2.12) são não-lineares; suas soluções podem ser obtidas numericamente.

\subsection{Inferência Sobre os Parâmetros $\alpha$ e $\beta$ ou Fun- ções dos Mesmos}

Para inferências sobre os parâmetros de um determinado modelo ou funções dos mesmos, usualmente, utiliza-se a aproximaçẫo Normal assintótica dos estimadores de máxima verossimilhança (ver por exemplo, Mood, Graybill e Boos, 1974). Assim consideramos, 


$$
(\hat{\alpha}, \hat{\beta}) \stackrel{a}{\sim} N\left\{(\alpha, \beta) ; I^{-1}(\hat{\alpha}, \hat{\beta})\right\}
$$

onde $I(\alpha, \beta)$ é a matriz de informação de Fisher dada pelas esperanças das segundas derivadas parciais de $\ell(\alpha, \beta)$ com respeito a $\alpha$ e $\beta$ a menos do sinal (ver por exemplo, Box e Tiao, 1973).

As segundas derivadas parciais da função log-verossimilhança $\ell(\alpha, \beta)(2.8)$ com respeito a $\alpha$ e $\beta$ são dadas por,

$$
\begin{gathered}
\frac{\partial^{2} \ell(\alpha, \beta)}{\partial \alpha^{2}}=-e^{\alpha} \sum_{i=1}^{k} V_{i} A_{i} e^{-\beta / V_{i}} \\
\frac{\partial^{2} \ell(\alpha, \beta)}{\partial \alpha \partial \beta}=e^{\alpha} \sum_{i=1}^{k} A_{i} e^{-\beta / V_{i}} \\
\frac{\partial^{2} \ell(\alpha, \beta)}{\partial \beta^{2}}=-e^{\alpha} \sum_{i=1}^{k} \frac{A_{i}}{V_{i}} e^{-\beta / V_{i}}
\end{gathered}
$$

Como estamos assumindo dados sob esquema de censura de tipo II, os $r_{i}$ são fixos, $i=1,2, \ldots, k, E\left(A_{i}\right)=\frac{r_{i}}{\lambda_{i}}$, onde $\lambda_{i}$ é dado em (2.6), isto é,

$$
E\left(A_{i}\right)=\frac{r_{i} e^{\beta / V_{i}}}{V_{i} e^{\alpha}}
$$

Substituindo a expressão (2.17) em (2.14) temos,

$$
E\left\{\frac{-\partial^{2} \ell(\alpha, \beta)}{\partial \alpha^{2}}\right\}=r
$$

em (2.15), 


$$
E\left\{\frac{-\partial^{2} \ell(\alpha, \beta)}{\partial \alpha \partial \beta}\right\}=-a_{1}
$$

e em (2.16),

$$
E\left\{\frac{-\partial^{2} \ell(\alpha, \beta)}{\partial \beta^{2}}\right\}=a_{2}
$$

onde $r=\sum_{i=1}^{k} r_{i}, a_{1}=\sum_{i=1}^{k} \frac{r_{i}}{V_{i}}$ e $a_{2}=\sum_{i=1}^{k} \frac{\pi}{V_{i}^{2}}$.

Portanto, a matriz de informação de Fisher para $\alpha$ e $\beta$ é dada por,

$$
I(\alpha, \beta)=\left[\begin{array}{cc}
r & -a_{1} \\
-a_{1} & \dot{a}_{2}
\end{array}\right]
$$

Assim, em (2.13),

$$
I^{-1}(\alpha, \beta)=\frac{1}{r a_{2}-a_{1}^{2}}\left[\begin{array}{cc}
a_{2} & a_{1} \\
a_{1} & r
\end{array}\right]
$$

isto é,

$$
\operatorname{var}(\hat{\alpha}) \cong \frac{a_{2}}{r a_{2}-a_{1}^{2}}, \operatorname{var}(\hat{\beta}) \cong \frac{r}{r a_{2}-a_{1}^{2}} \mathrm{e} \operatorname{cov}(\hat{\alpha}, \hat{\beta}) \cong \frac{a_{1}}{r a_{2}-a_{1}^{2}}
$$

Observe que os elementos da matriz (2.18) são constantes, o que implica numa boa aproximação Normal assintótica para os estimadores de máxima verossimilhança, $\hat{\alpha}$ e $\hat{\beta}$ (ver por exemplo, Sprott, 1973 e 1980), e as inferências sobre $\alpha$ e $\beta$ podem ser baseadas em (2.13).

Usualmente, indústrias de várias áreas têm interese em inferências sobre o tempo médio de sobrevivência, $\theta_{1}$, sob o nível usual de funcionamento, $V_{1}$. Um procedimento de inferência sobre esse parâmetro é a utilização do chamado método “Delta" (ver por exemplo, Miller, 1981; Apêndice 1). 
Considerando as expressões (2.2) e (2.6), sob o nivel de estresse usual, $V_{1}, \theta_{1}=g(\alpha, \beta)=e^{\beta / V_{1}} / V_{1} e^{\alpha}, \partial g / \partial \alpha=-e^{\beta / V_{1}} / V_{1} e^{\alpha}$ e $\partial g / \partial \beta=e^{\beta / V_{1}} / V_{1}^{2} e^{\alpha}$.

Pelo método "Delta", o estimador de máxima verossimilhança de $\theta_{1}=$ $g(\alpha, \beta)$ tem uma distribuição Normal assintótica, dada por,

$$
\hat{\theta}_{1} \stackrel{a}{\sim} N\left\{\frac{e^{\beta / V_{1}}}{V_{1} e^{\alpha}} ; \hat{\sigma}_{\theta_{1}}^{2}\right\}
$$

onde

$$
\begin{gathered}
\hat{\sigma}_{\theta_{1}}^{2}=\frac{a_{2}}{r a_{2}-a_{1}^{2}}\left(\frac{\partial g}{\partial \alpha}\right)_{(\hat{\alpha}, \hat{\beta})}^{2}+2 \frac{a_{1}}{r a_{2}-a_{1}^{2}}\left(\frac{\partial g}{\partial \alpha}\right)_{(\hat{\alpha}, \hat{\beta})}\left(\frac{\partial g}{\partial \beta}\right)_{(\hat{\alpha}, \hat{\beta})}+\frac{r}{r a_{2}-a_{1}^{2}}\left(\frac{\partial g}{\partial \beta}\right)_{(\hat{\alpha}, \hat{\beta})}^{2} \\
\text { O estimador de máxima verossimilhança de } \theta_{1} \text { é dado por, } \\
\hat{\theta}_{1}=e^{\hat{\beta} / V_{1}} / V_{1} e^{\hat{\alpha}} .
\end{gathered}
$$

Também,

$$
\left(\frac{\partial g}{\partial \alpha}\right)_{(\hat{\alpha}, \hat{\beta})}=-e^{\hat{\beta} / V_{1}} / V_{1} e^{\hat{\alpha}}=-\hat{\theta}_{1} \text { e }\left(\frac{\partial g}{\partial \beta}\right)_{(\hat{\alpha}, \hat{\beta})}=e^{\hat{\beta} / V_{1}} / V_{1}^{2} e^{\hat{\alpha}}=\hat{\theta}_{1} / V_{1} .
$$

Portanto,

$$
\hat{\sigma}_{\theta_{1}}^{2}=\frac{a_{2} \hat{\theta}_{1}^{2}}{r a_{2}-a_{1}^{2}}+\frac{r \hat{\theta}_{1}^{2}}{V_{1}^{2}\left(r a_{2}-a_{1}^{2}\right)}-2 \frac{a_{1} \hat{\theta}_{1}^{2}}{V_{1}\left(r a_{2}-a_{1}^{2}\right)},
$$

isto é,

$$
\hat{\sigma}_{\theta_{1}}^{2}=\frac{\hat{\theta}_{1}^{2}}{\left(r a_{2}-a_{1}^{2}\right)}\left\{a_{2}-2 \frac{a_{1}}{V_{1}}+\frac{r}{V_{1}^{2}}\right\}
$$


Antes de utilizarmos a aproximação Normal assintótica (2.20), para inferências sobre $\theta_{1}$, devemos estudar com mais detalhes sua precisão, o que será feito na seção seguinte.

\subsection{O Problema da Inferência sobre $\theta_{1}$ Usando a Normalidade Assintótica dos Estimadores de Máxima Verossimilhança}

O uso da aproximação Normal assintótica dos estimadores de máxima verossimilhança é um dos procedimentos mais difundidos na aplicação de técnicas estatísticas em problemas práticos. É importante salientar que sempre devemos verificar se essa aproximação é viável, estudando sua precisão.

Primeiramente, podemos reescrever a função de verossimilhança (2.7), originalmente expressa em termos $\lambda_{i}$ para a relação estresse-resposta representada pelo modelo de Eyring (2.6) com parâmetros $\alpha$ e $\beta$, em termos dos parâmetros $\theta_{1}$ e $\beta$.

Explicitamente, de (2.2) e (2.6), sob o nível de estresse usual, $\theta_{1}=e^{\beta / V_{1}} / V_{1} e^{\alpha}$, isto é

$$
\alpha=\log \left\{\frac{e^{\beta / V_{1}}}{\theta_{1} V_{1}}\right\}
$$

substituindo $\alpha$ por (2.22) na função de verossimilhança (2.7) obtemos a função de verossimilhança para $\theta_{1}$ e $\beta$, dada por,

$$
L\left(\theta_{1}, \beta\right) \propto\left\{\frac{e^{\beta / V_{1}}}{\theta_{1} V_{1}}\right\}^{r} \exp \left\{-\beta a_{1}-\frac{e^{\beta / V_{1}}}{\theta_{1} V_{1}} \sum_{i=1}^{k} V_{i} A_{i} e^{-\beta / V_{i}}\right\}
$$

isto é, 


$$
L\left(\theta_{1}, \beta\right) \propto \theta_{1}^{-r} \exp \left\{-\beta\left(a_{1}-\frac{r}{V_{1}}\right)-\frac{1}{\theta_{1}} \sum_{i=1}^{k} \frac{V_{i}}{V_{1}} A_{i} e^{-\beta\left(\frac{1}{V_{i}}-\frac{1}{V_{1}}\right)}\right\} .
$$

A função log-verossimilhança para $\theta_{1}$ e $\beta$ é dada por,

$$
\ell\left(\theta_{1}, \beta\right) \propto-r \log \theta_{1}-\beta\left(a_{1}-\frac{r}{V_{1}}\right)-\frac{1}{\theta_{1}} \sum_{i=1}^{k} \frac{V_{i}}{V_{1}} A_{i} e^{-\beta\left(\frac{1}{V_{i}}-\frac{1}{v_{1}}\right)}
$$

Derivando $\ell\left(\theta_{1}, \beta\right)$ com respeito a $\theta_{1}$ e $\beta$, e igualando a zero, isto é, $\partial \ell / \partial \theta_{1}=0$ e $\partial \ell / \partial \beta=0$, obtemos os estimadores de máxima verossimilhança de $\theta_{1}^{\circ}$ e $\beta$ dados por,

$$
\hat{\theta}_{1}=\frac{1}{r} \sum_{i=1}^{k} \frac{V_{i}}{V_{1}} A_{i} \exp \left\{-\hat{\beta}\left[\frac{1}{V_{i}}-\frac{1}{V_{1}}\right]\right\}
$$

e $\hat{\beta}$ que é a solução da equação

$$
\frac{\sum_{i=1}^{k} V_{i} A_{i}\left(\frac{1}{V_{i}}-\frac{1}{V_{1}}\right) \exp \left\{-\hat{\beta}\left(\frac{1}{V_{i}}-\frac{1}{V_{1}}\right)\right\}}{\sum_{i=1}^{k} V_{i} A_{i} \exp \left\{-\hat{\beta}\left(\frac{1}{V_{i}}-\frac{1}{V_{1}}\right)\right\}}=\frac{a_{1}}{r}-\frac{1}{V_{1}}
$$

Por analogia ao procedimento adotado na seção (2.4), inferências sobre os parâmetros $\theta_{1}$ e $\beta$ são baseadas na aproximação assintótica Normal dos estimadores de máxima verossimilhança $\hat{\theta_{1}}$ e $\hat{\beta}$, dada por,

$$
\left(\hat{\theta_{1}}, \hat{\beta}\right) \stackrel{a}{\sim} N\left\{\left(\theta_{1}, \beta\right) ; I^{-1}\left(\hat{\theta_{1}}, \hat{\beta}\right)\right\}
$$

onde $I\left(\theta_{1}, \beta\right)$ é a matriz de informação de Fisher nos parâmetros $\theta_{1}$ e $\beta$.

Calculando as segundas derivadas parciais da função log-verossimilhança (2.24) temos, 


$$
\begin{gathered}
\frac{\partial^{2} \ell\left(\theta_{1}, \beta\right)}{\partial \theta_{1}^{2}}=\frac{r}{\theta_{1}^{2}}-\frac{2}{\theta_{1}^{3}} \sum_{i=1}^{k} \frac{V_{i}}{V_{1}} A_{i} \exp \left\{-\beta\left(\frac{1}{V_{i}}-\frac{1}{V_{1}}\right)\right\}, \\
\frac{\partial^{2} \ell\left(\theta_{1}, \beta\right)}{\partial \theta_{1} \partial \beta}=-\frac{1}{\theta_{1}^{2}} \sum_{i=1}^{k} \frac{V_{i}}{V_{1}} A_{i}\left(\frac{1}{V_{i}}-\frac{1}{V_{1}}\right) \exp \left\{-\beta\left(\frac{1}{V_{i}}-\frac{1}{V_{1}}\right)\right\},
\end{gathered}
$$

e

$$
\frac{\partial^{2} \ell\left(\theta_{1}, \beta\right)}{\partial \beta^{2}}=-\frac{1}{\theta_{1}} \sum_{i=1}^{k} \frac{V_{i}}{V_{1}} A_{i}\left(\frac{1}{V_{i}}-\frac{1}{V_{1}}\right)^{2} \exp \left\{-\beta\left(\frac{1}{V_{i}}-\frac{1}{V_{1}}\right)\right\}
$$

Sabendo-se que $E\left(A_{i}\right)=r_{i} / \lambda_{i}, \lambda_{i}=V_{i} \exp \left\{\alpha-\beta / V_{i}\right\}$ e $\alpha=\log \left(e^{\beta / V_{1}} / \theta_{1} V_{1}\right)$, obtém-se,

$$
E\left(A_{i}\right)=\frac{r_{i} \theta_{1} V_{1}}{V_{i} \exp \left\{-\beta\left(\frac{1}{V_{i}}-\frac{1}{V_{1}}\right)\right\}}
$$

Dessa forma, a matriz de informação de Fisher para $\theta_{1}$ e $\beta$ é dada por

$$
I\left(\theta_{1}, \beta\right)=\left[\begin{array}{cc}
\frac{r}{\theta_{1}^{2}} & \frac{b_{1}}{\theta_{1}} \\
\frac{b_{1}}{\theta_{1}} & b_{2}
\end{array}\right],
$$

onde

$b_{1}=\sum_{i=1}^{k} r_{i}\left(1 / V_{i}-1 / V_{1}\right)=a_{1}-r / V_{1}$ e $b_{2}=\sum_{i=1}^{k} r_{i}\left(1 / V_{i}-1 / V_{1}\right)^{2}=a_{2}-2 a_{1} / V_{1}+r / V_{1}^{2}$,

e em (2.27),

$$
I^{-1}\left(\theta_{1}, \beta\right)=\frac{\theta_{1}^{2}}{r b_{2}-b_{1}^{2}}\left[\begin{array}{cc}
b_{2} & -\frac{b_{1}}{\theta_{1}} \\
-\frac{b_{1}}{\theta_{1}} & \frac{r}{\theta_{1}^{2}}
\end{array}\right]
$$


A distribuição assintótica do estimador de máxima verossimilhança para $\theta_{1}$ é dada (de (2.27)) por,

$$
\hat{\theta_{1}} \stackrel{a}{\sim} N\left(\theta_{1}, \hat{\sigma}_{\theta_{1}}^{2}\right)
$$

onde

$$
\hat{\sigma}_{\theta_{2}}=\frac{b_{2} \hat{\theta}_{1}^{2}}{r b_{2}-b_{1}^{2}}=\frac{\left(a_{2}-2 \frac{a_{1}}{V_{1}}+\frac{r}{V_{1}^{2}}\right) \hat{\theta}_{1}^{2}}{r\left(a_{2}-2 \frac{a_{1}}{V_{1}}+\frac{r}{V_{1}^{2}}\right)-\left(a_{1}-\frac{r}{V_{1}}\right)^{2}} .
$$

Portanto,

$$
\hat{\sigma}_{\theta_{1}}^{2}=\frac{\hat{\theta}_{1}^{2}}{r a_{2}-a_{1}^{2}}\left\{a_{2}-2 \frac{a_{1}}{V_{1}}+\frac{r}{V_{1}^{2}}\right\}
$$

Observamos que a variância assintótica de $\hat{\theta}_{1}(2.30)$ é igual à obtida em (2.21).

Considerando a matriz de informação de Fisher (2.28) observamos que seus elementos não são constantes (dependem de $\theta_{1}$ ), implicando numa aproximação assintótica Normal, em geral, imprecisa para os estimadores de máxima verossimilhança de $\theta_{1}$ e $\beta$ para amostras pequenas e moderadas (ver por exemplo, Sprott, $1973,1980)$. Assim, usando a aproximação Normal (2.20), obtida através do uso do método "Delta" (ver Apêndice 1), em geral, não obtemos boas inferências para $\theta_{1}$, um argumento forte para procurarmos outra parametrização, buscando uma melhor aproximação assintótica Normal da função de verossimilhança para esse parâmetro.

\subsection{O Estudo de Diferentes Parametrizações}

Com o objetivo de melhorarmos as inferências sobre $\theta_{1}$, consideramos nesta seção, o estudo de diferentes parametrizações visando a obtenção de funções de 
verossimilhanças aproximadamente Normais.

\subsubsection{Reparametrização Ortogonal: Ortogonalização em $\beta$}

A reparametrização Ortogonal (ver por exemplo, Cox e Reid, 1987) é uma reparametrização que diagonaliza a matriz de informação de Fisher para os parâmetros $\theta_{1}$ e $\beta$ dada em (2.28). Com isso, obtemos um estimador de $\beta$ estável com respeito a $\theta_{1}$.

Para obtermos tal parametrização, transformamos os parâmetros originais $\theta_{1}$ e $\beta$, onde $\theta_{1}$ é o parâmetro de interesse e $\beta$ o parâmetro "nuisance", nos parâmetros ortogonais $\theta_{1}$ e $\gamma$, resolvendo a equação diferencial,

$$
-I_{\theta_{1}, \beta}=I_{\beta, \beta} \frac{\partial \beta}{\partial \theta_{1}}
$$

onde $I_{\beta, \beta}$ e $I_{\theta_{1}, \beta}$ são os éléfhèntos da matriz de informação de Fisher (2.28) com respeito a $\beta$ e ao produto cruzado $\left(\theta_{1}, \beta\right)$, respectivamente.

Substituindo os elementos de (2.28) na equação diferencial (2.31), temos,

$$
-\frac{b_{1}}{\theta_{1}}=b_{2} \frac{\partial \beta}{\partial \theta_{1}}
$$

isto é,

$$
-b_{1} \frac{d \theta_{1}}{\theta_{1}}=b_{2} d \beta
$$

e, uma solução é dada por,

$$
-\frac{b_{1}}{b_{2}} \log \left(\theta_{1}\right)+c(\gamma)=\beta,
$$


onde $c(\gamma)$ é uma função arbitrária de $\gamma$.

Considerando $c(\gamma)=\gamma$ temos que,

$$
\beta=\gamma-\frac{b_{1}}{b_{2}} \log \left(\theta_{1}\right)
$$

isto é,

$$
\gamma=\beta+\frac{b_{1}}{b_{2}} \log \left(\theta_{1}\right)
$$

substituindo (2.32) em (2.23), a nova função de verossimilhança em termos dos parâmetros ortogonais, $\theta_{1}$ e $\gamma$, é dada por,

$$
\begin{gathered}
L\left(\theta_{1}, \gamma\right) \propto \theta_{1}^{-r} \exp \left\{-\left[\gamma-\frac{b_{1}}{b_{2}} \log \left(\theta_{1}\right)\right]\left[a_{1}-\frac{r}{V_{1}}\right]\right\} . \\
\gamma^{\prime} \exp \left\{-\frac{1}{\theta_{2}} \sum_{i=1}^{k} \frac{V_{i}}{V_{1}} A_{i} \exp \left[-\left(\gamma-\frac{b_{1}}{b_{2}} \log \left(\theta_{1}\right)\right)\left(\frac{1}{V_{i}}-\frac{1}{V_{1}}\right)\right]\right\} .
\end{gathered}
$$

Portanto,

$$
L\left(\theta_{1}, \gamma\right) \propto \theta_{1}^{\frac{b_{1}}{b_{2}}-r} \exp \left\{-\gamma^{b_{1}}-\sum_{i=1}^{k} \frac{V_{i}}{V_{1}} A_{i} e^{-\gamma\left(\frac{1}{V_{i}}-\frac{1}{V_{1}}\right)} \theta_{1}^{\frac{b_{1}}{b_{2}}\left(\frac{1}{V_{i}}-\frac{1}{V_{1}}\right)-1}\right\}
$$

onde

$$
r=\sum_{i=1}^{k} r_{i}, b_{1}=\sum_{i=1}^{k} r_{i}\left(1 / V_{i}-1 / V_{1}\right)=a_{1}-r / V_{1}
$$

e

$$
b_{2}=\sum_{i=1}^{k} r_{i}\left(1 / V_{i}-1 / V_{1}\right)^{2}=a_{2}-2 a_{1} / V_{1}+r / V_{1}^{2}\left(a_{1}=\sum_{i=1}^{k} r_{i} / V_{i} \text { e } a_{2}=\sum_{i=1}^{k} r_{i} / V_{i}^{2}\right)
$$


Considerando o logarítmo de (2.34), $\ell\left(\theta_{1}, \gamma\right)$, e as equações $\partial \ell / \partial \theta_{1}=0 \mathrm{e}$ $\partial \ell / \partial \gamma=0$, os estimadores de máxima verossimilhança para $\theta_{1}$ e $\gamma$ satisfazem o seguinte sistema de equações:

$$
\begin{gathered}
\sum_{i=1}^{k} \frac{V_{i}}{V_{1}} A_{i}\left[1-\frac{b_{1}}{b_{2}}\left(\frac{1}{V_{i}}-\frac{1}{V_{1}}\right)\right] e^{-\hat{\gamma}\left(\frac{1}{V_{i}}-\frac{1}{V_{1}}\right)} \hat{\theta}_{1}^{\frac{b_{1}}{b_{2}}\left(\frac{1}{V_{i}}-\frac{1}{V_{1}}\right)-1}=r-\frac{b_{1}^{2}}{b_{2}} \\
\sum_{i=1}^{k} \frac{V_{i}}{V_{1}} A_{i}\left(\frac{1}{V_{i}}-\frac{1}{V_{1}}\right) e^{-\hat{\gamma}\left(\frac{1}{V_{i}}-\frac{1}{V_{1}}\right)} \hat{\theta}_{1}^{\frac{b_{1}}{b_{2}}\left(\frac{1}{V_{i}}-\frac{1}{V_{1}}\right)-1}=b_{1} .
\end{gathered}
$$

Como $E\left(A_{i}\right)=\theta_{1} V_{1} r_{i} / V_{i} \exp \left\{-\beta\left(1 / V_{i}-1 / V_{1}\right)\right\}$, onde $\beta=\gamma-\left(b_{1} / b_{2}\right) \log \left(\theta_{1}\right)$, temos,

$$
E\left(A_{i}\right)=\frac{r_{i} V_{1} e_{1}^{-\frac{b_{1}}{b_{2}}\left(\frac{1}{v_{i}}-\frac{1}{V_{1}}\right)+1}}{V_{i} e^{-\gamma\left(\frac{1}{v_{i}}-\frac{1}{V_{1}}\right)}}
$$

Calculando-se as segundas derivadas parcias de $\ell\left(\theta_{1}, \gamma\right), \partial^{2} \ell / \partial \theta_{1}^{2}, \partial^{2} \ell / \partial \theta_{1} \partial \gamma$ e $\partial^{2} \ell / \partial \gamma^{2}$, e substituindo-se $E\left(A_{i}\right)$ dada acima, seus valores esperados a menos do sinal são dados por,

$$
E\left\{-\frac{\partial^{2} \ell}{\partial \theta_{1}^{2}}\right\}=\frac{c_{1}}{\theta_{1}^{2}}
$$

onde

$$
\begin{aligned}
& c_{1}=\left[\frac{b_{1}}{b_{2}}\left(a_{1}-\frac{r}{V_{1}}\right)-r\right]+\sum_{i=1}^{k} r_{i}\left[1-\frac{b_{1}}{b_{2}}\left(\frac{1}{V_{i}}-\frac{1}{V_{1}}\right)\right]\left[2-\frac{b_{1}}{b_{2}}\left(\frac{1}{V_{i}}-\frac{1}{V_{1}}\right)\right] \\
& =r-\frac{b_{1}^{2}}{b_{2}} ; \\
& \vdots \\
& \quad E\left\{\frac{-\partial^{2} \ell}{\partial \theta_{1} \partial \gamma}\right\}=\frac{1}{\theta_{1}} \sum_{i=1}^{k} r_{i}\left(\frac{1}{V_{i}}-\frac{1}{V_{1}}\right)\left[1-\frac{b_{1}}{b_{2}}\left(\frac{1}{V_{i}}-\frac{1}{V_{1}}\right)\right]=0 ;
\end{aligned}
$$




$$
E\left\{\frac{-\partial^{2} \ell}{\partial \gamma^{2}}\right\}=\sum_{i=1}^{k} r_{i}\left(\frac{1}{V_{i}}-\frac{1}{V_{1}}\right)^{2}=b_{2}
$$

Portanto, a matriz de informação de Fisher para $\theta_{1}$ e $\gamma$ é dada por,

$$
I\left(\theta_{1}, \gamma\right)=\left[\begin{array}{cc}
\frac{r-b_{1}^{2} / b_{2}}{\theta_{1}^{2}} & 0 \\
0 & b_{2}
\end{array}\right]
$$

Usando a aproximação assintótica do estimador de máxima verossimilhança de $\theta_{1}$, temos que $\hat{\theta}_{1}$ tem uma distribuição assintótica dada por,

$$
\hat{\theta_{1}} \stackrel{2}{\sim} N\left(\theta_{1}, \hat{\sigma}_{\theta_{1}}^{2}\right)
$$

onde

$$
\hat{\sigma}_{\theta_{1}}^{2}=\frac{b_{2} \hat{\theta}_{1}^{2}}{r b_{2}-b_{1}^{2}}
$$

isto é,

$$
\hat{\sigma}_{\theta_{1}}^{2}=\frac{\hat{\theta}_{1}^{2}}{r a_{2}-a_{1}^{2}}\left\{a_{2}-2 \frac{a_{1}}{V_{1}}+\frac{r}{V_{1}^{2}}\right\}
$$

Observamos que, apesar de termos uma parametrização ortogonal (matriz (2.37) diagonal) (ver por exemplo, Cox e Reid, 1987), como os elementos de $I\left(\theta_{1}, \gamma\right)$ em (2.37) não são constantes, a ortogonalização em $\beta$ pode não melhorar a normalidade assintótica do estimador de máxima verossimilhança de $\theta_{1}$ (ver por exemplo; Sprott, 1973, 1980). 


\subsubsection{Uma Proposta para Obtenção de Estimadores de $\alpha$ e $\beta$ Assintoticamente Independentes}

Visando a obtenção de estimadores assintoticamente independentes para os parâmetros $\alpha$ e $\beta$, Mann Schaffer e Singpurwalla (1974) sugerem um pequeno melhoramento na relação estresse-resposta (2.6), sem modificar sua caracteristica básica, isto é, definindo,

$$
\bar{V}=\frac{\sum_{i=1}^{k} \frac{r_{i}}{V_{i}}}{\sum_{i=1}^{k} r_{i}}
$$

como a média ponderada dos $V_{i}^{-1}{ }^{\prime} s$, o modelo de Eyring com uma variável estresse (2.6) pode ser reescrito na forma,

$$
\lambda_{i}=V_{i} \exp \left\{\alpha-\beta\left(V_{i}^{-1}-\bar{V}\right)\right\} .
$$

Considerando-se a expressão (2.40) e a função de verossimilhança (2.5), a nova função de verossimilhança para $\alpha$ e $\beta$ é dada por,

$$
L(\alpha, \beta) \propto \exp \left\{\alpha r-\beta d_{1}-e^{\alpha} \sum_{i=1}^{k} V_{i} A_{i} e^{-\beta\left(V_{i}^{-1}-\bar{Y}\right)}\right\}
$$

onde $d_{1}=\sum_{i=1}^{k} r_{i}\left(V_{i}^{-1}-\bar{V}\right)$.

Observando que $d_{1}=0$, temos,

$$
L(\alpha, \beta) \propto \exp \left\{\alpha r-e^{\alpha} \sum_{i=1}^{k} V_{i} A_{i} e^{-\beta\left(V_{i}^{-1}-\bar{V}\right)}\right\}
$$

(observe que (2.41) poderia ser obtida via reparametrização ortogonal (ver por exemplo, Cox e Reid, 1987) adotada para o parâmetro $\alpha$ resolvendo-se a equação dife- 
rencial $-I_{\alpha, \beta}=I_{\alpha, \alpha} \frac{\partial \alpha}{\partial \beta}$, onde $I_{\alpha, \beta}$ e $I_{\alpha, \alpha}$ são os elementos de (2.18) com respeito a $\alpha$ e ao produto cruzado $(\alpha, \beta))$.

Os estimadores de máxima verossimilhança de $\alpha$ e $\beta$, obtidos resolvendo-se as equações $\partial \ell(\alpha, \beta) / \partial \alpha=0$ e $\partial \ell(\alpha, \beta) / \partial \beta=0$, são dados por,

$$
\hat{\alpha}=\log \left[\frac{r}{\sum_{i=1}^{k} V_{i} A_{i} e^{-\hat{\beta}\left(V_{i}^{-1}-\bar{V}\right)}}\right]
$$

e $\hat{\beta}$ é a solução da equação

$$
\sum_{i=1}^{k} V_{i}\left(V_{i}^{-1}-\bar{V}\right) A_{i} e^{-\hat{\beta}\left(V_{i}^{-1}-\bar{V}\right)}=0
$$

Considerando a relação estresse-resposta (2.40), obtemos,

17

$$
E\left(A_{i}\right)=\frac{r_{i} \exp \left\{\beta\left(V_{i}^{-1}-\bar{V}\right)\right\}}{V_{i} e^{\alpha}}
$$

As esperanças das, segundas derivadas parciais $\partial^{2} \ell(\alpha, \beta) / \partial \alpha^{2}$, $\partial^{2} \ell(\alpha, \beta) / \partial \alpha \partial \beta$ e $\partial^{2} \ell(\alpha, \beta) / \partial \beta^{2}$, a menos do sinal, são dadas por,

$$
\begin{gathered}
E\left\{\frac{-\partial^{2} \ell}{\partial \alpha^{2}}\right\}=r \\
E\left\{\frac{-\partial^{2} \ell}{\partial \alpha \partial \beta}\right\}=-\sum_{i=1}^{k} r_{i}\left(V_{i}^{-1}-\bar{V}\right)=0
\end{gathered}
$$

e

$$
E\left\{\frac{-\partial^{2} \ell}{\alpha \beta^{2}}\right\}=\sum_{i=1}^{k} r_{i}\left(V_{i}^{-1}-\bar{V}\right)^{2}=d_{1}=a_{2}-\frac{a_{1}^{2}}{r}
$$


A matriz de informação de Fisher com respeito a $\alpha$ e $\beta$ é dada por,

$$
I(\alpha, \beta)=\left[\begin{array}{cc}
r & 0 \\
0 & a_{2}-\frac{a_{1}^{2}}{r}
\end{array}\right] .
$$

Podemos observar que a matriz (2.44) é diagonal e constante, dessa forma, os parâmetros são ortogonais e os estimadores de máxima verossimilhança de $\alpha$ e $\beta$ sãs distribuídos assintoticamente como variáveis aleatórias Normais (ver por exemplo, Sprott, 1973, 1980), isto é, $\hat{\alpha}$ e $\hat{\beta}$ são assintoticamente independentes. Assim,

$$
(\hat{\alpha}, \hat{\beta}) \stackrel{a}{\sim} N\left\{(\alpha, \beta) ; I^{-1}(\hat{\alpha}, \hat{\beta})\right\}
$$

onde

$$
I^{-1}(\alpha, \beta)=\left[\begin{array}{cc}
\frac{1}{r} & 0 \\
0 & \frac{r}{r a_{2}-a_{1}^{2}}
\end{array}\right]
$$

isto é, $\operatorname{var}(\hat{\alpha}) \cong 1 / r, \operatorname{var}(\hat{\beta}) \cong r /\left(r a_{2}-a_{1}^{2}\right)$ e $\operatorname{cov}(\hat{\alpha}, \hat{\beta}) \cong 0$.

Como o objetivo é inferir sobre o tempo médio de sobrevivência, $\theta_{1}$, sob o nivel de estresse usual, $V_{1}, \operatorname{de}(2.40), \theta_{1}=g(\alpha, \beta)=\exp \left\{\beta\left(V_{1}^{-1}-\bar{V}\right)\right\} / V_{1} e^{\alpha}, \partial g / \partial \alpha=$ $-\exp \left\{\beta\left(V_{1}^{-1}-\bar{V}\right)\right\} / V_{1} e^{\alpha}$ e $\partial g / \partial \beta=\left(V_{1}^{-1}-\bar{V}\right) \exp \left\{\beta\left(V_{1}^{-1}-\bar{V}\right)\right\} / V_{1} e^{\alpha}$. Pelo método "Delta" (ver por exemplo, Miller, 1981; Apêndice 1), o estimador de máxima verossimilhança de $\theta_{1}=g(\alpha, \beta)$ tem uma distribuição assintótica dada por,

$$
\hat{\theta}_{1} \stackrel{a}{\sim} N\left\{\theta_{1}, \hat{\sigma}_{\theta_{1}}^{2}\right\}
$$

onde 


$$
\begin{gathered}
\hat{\sigma}_{\theta_{1}}^{2}=(1 / r)(\partial g / \partial \alpha)_{(\hat{\alpha}, \hat{\beta})}^{2}+\left(r /\left(r a_{2}-a_{1}^{2}\right)\right)(\partial g / \partial \beta)_{(\hat{\alpha}, \hat{\beta})}^{2}, \text { mas, } \\
(\partial g / \partial \alpha)_{(\hat{\alpha}, \hat{\beta})}=-\hat{\theta}_{1}, \text { e }(\partial g / \partial \beta)_{(\hat{\alpha}, \hat{\beta})}=\left(V_{1}^{-1}-\bar{V}\right)_{\hat{\theta}_{1}} .
\end{gathered}
$$

Daí,

$$
\hat{\sigma}_{\theta_{1}}^{2}=\frac{\theta_{1}^{2}}{r a_{2}-a_{1}^{2}}\left\{a_{2}-\frac{a_{1}^{2}}{r}+r\left(V_{1}^{-1}-\bar{V}\right)^{2}\right\}
$$

Observando que $\bar{V}=a_{1} / r$,

$$
\hat{\sigma}_{\theta_{1}}^{2}=\frac{\theta_{1}^{2}}{r a_{2}-a_{1}^{2}}\left\{a_{2}+\frac{r}{V_{1}^{2}}-2 \frac{a_{1}}{V_{1}}\right\}
$$

Para verificar se é boa a precisão da aproximação Normal assintótica do estimador de máxima verossimilhança para o parâmetro $\theta_{1}$ considerando-se a sugestão de Mann, Schaffer e Singpurwalla (1974) (ver (2.39)), reescrevemos a função de verossimilhança de $\alpha$ e $\beta$ (2.41) em termos dos parâmetros $\theta_{1}$ e $\beta$, isto é, considerando -se a expressão (2.40) sob o nível de estresse usual, $V_{1}$, obtemos,

$$
\alpha=\log \left\{\frac{e^{\mathcal{\beta}\left(V_{1}^{-1}-\bar{V}\right)}}{\theta_{1} V_{1}}\right\}
$$

Substituindo $\alpha$ dado acima em (2.41) obtemos a expressão da função de verossimilhança para $\theta_{1} e \beta$ igual a expressão (2.23), isto é,

$$
L\left(\theta_{1}, \beta\right) \propto \theta_{1}^{-r} \exp \left\{-\beta\left(a_{1}-\frac{r}{V_{1}}\right)-\frac{1}{\theta_{1}} \sum_{i=1}^{k} \frac{V_{i}}{V_{1}} A_{i} e^{-\beta\left(\frac{1}{v_{i}}-\frac{1}{V_{1}}\right)}\right\}
$$


Dessa forma, como discutido na seção 2.5 , a aproximação Normal assintótica do estimador de máxima verossimilhança $\hat{\theta_{1}}$, obtida utilizando-se o método "Delta" (ver Apêndice 1) não terá boa precisão.

0 fato é que, para todas as parametrizações adotadas, encontramos a mesma variância assintótica, $\hat{\sigma}_{\theta_{1}}^{2}$ - ver (2.21), (2.30), (2.38) e (2.45) - baseada na aproximação Normal assintótica do estimador de máxima verossimilhança para $\theta_{1}$. Isto significa que, se a aproximação não for precisa, as inferências sobre $\theta_{1}$ serão ruins, independentemente da parametrização considerada. Também observamos que uma boa aproximação Normal assintótica para a função de verossimilhança para $\alpha$ e. $\beta$ não implica em boa aproximação Normal para o estimador de verossimilhança de $\theta_{1}$ (função não-linear de $\alpha$ e $\beta$ ) sendo necessário muito cuidado em utilizá-la nas inferências sobre esse parâmetro.

\subsubsection{Reparametrização Logarítmica em $\theta_{1}$}

Considerando a função de verossimilhança para $\theta_{1}$ e $\beta(2.23)$ e a reparametrização $\delta=\log \theta_{1}$, isto é, $\theta_{1}=e_{1}^{\delta}$, a função de verossimilhança na parametrização $\delta$ e $\beta$ é dada por,

$$
L(\delta, \beta) \propto e^{-\delta r} \exp \left\{-\beta\left(a_{1}-\frac{r}{V_{1}}\right)-e^{-\delta} \sum_{i=1}^{k} \frac{V_{i}}{V_{1}} A_{i} e^{-\beta\left(\frac{1}{V_{i}}-\frac{1}{V_{1}}\right)}\right\}
$$

Resolvendo as equações as equações $\partial \ell(\delta, \beta) / \partial \delta=0$ e $\partial \ell(\delta, \beta) / \partial \beta=0$ com respeito a $\delta$ e $\beta$, obtemos os estimadores de máxima verossimilhança

$$
\hat{\delta}=\log \left[\frac{\sum_{i=1}^{k} \frac{V_{i}}{V_{1}} A_{i} e^{-\hat{\beta}\left(\frac{1}{V_{i}}-\frac{1}{V_{1}}\right)}}{r}\right]
$$


e $\hat{\beta}$ que é a solução da equação

$$
\frac{\sum_{i=1}^{k} \frac{V_{i}}{V_{1}} A_{i}\left(\frac{1}{V_{i}}-\frac{1}{\left.V_{1}\right)} e^{-\hat{\beta}\left(\frac{1}{V_{i}}-\frac{1}{V_{1}}\right)}\right.}{\sum_{i=1}^{k} \frac{V_{i}}{V_{1}} A_{i} e^{-\hat{\beta}\left(\frac{1}{V_{i}}-\frac{1}{V_{2}}\right)}}=\frac{a_{1}}{r}-\frac{1}{V_{1}} .
$$

Como $E\left(A_{i}\right)=r_{i} V_{1} e^{\delta} / V_{i} \exp \left\{-\beta\left(1 / V_{i}-1 / V_{1}\right)\right\}$, a matriz de informação de Fisher para $\delta$ e $\beta$ é dada por,

$$
I(\delta, \beta)=\left[\begin{array}{ll}
r & b_{1} \\
b_{1} & b_{2}
\end{array}\right],
$$

onde, $b_{1}=\sum_{i=1}^{k} r_{i}\left(1 / V_{i}-1 / V_{1}\right)$ e $b_{2}=\sum_{i=1}^{k} r_{i}\left(1 / V_{i}-1 / V_{1}\right)^{2}$.

Os elementos da matriz (2.49) são constantes. Portanto, a aproximação Normal assintótica para a função de verossimilhança para $\delta$ e $\beta$ tem boa precisão (ver por exemplo, Sprott, 1973, 1980).

Daí, o estimador de máxima verossimilhança $\hat{\delta}$, tem uma distribuição assintótica dada por,

$$
\hat{\delta} \stackrel{a}{\sim} N\left(\delta, \hat{\sigma}_{\delta}^{2}\right)
$$

onde $\hat{\sigma}_{\delta}^{2}$ é o elemento da inversa da matriz $I(\delta, \beta)$, em (2.49), com respeito a $\delta$, isto é,

$$
\hat{\sigma}_{\delta}^{2}=\frac{b_{2}}{r b_{2}-b_{1}^{2}}=\frac{1}{r a_{2}-a_{1}^{2}}\left(a_{2}-2 \frac{a_{1}}{V_{1}}+\frac{r}{V_{1}^{2}}\right),
$$

e inferências baseadas em (2.50) terão boa precisão. 
Considerando o nosso objetivo inicial, ou seja, obtenção de uma parametrização onde a aproximação Normal assintótica para os estimadores de máxima verossimilhança dos parâmetros envolvidos tenha boa precisão, o estudo estaria finalizado. Porém uma parametrização desejável é aquela que propicia, além de uma boa aproximação Normal assintótica da função de verossimilhança dos parâmetros envolvidos, parâmetros ortogonais (não correlacionados), isto é, uma parametrização assintóticamente independente.

$\mathrm{Na}$ seção seguinte essa parametrização é obtida via reparametrização ortogonal (ver por exemplo, Cox e Reid, 1987) associada a uma reparametrização logarítmica.

\subsubsection{Parametrização Assintoticamente Independente}

Para obtermos uma parametrização assintoticamente independente, inicialmente exploraremos o uso da reparantetriziação ortogonal (ver por exemplo, Cox e Reid, 1987) para ortogonalizar o parâmetro $\theta_{1}$, isto é, neste caso, consideramos $\beta$ o parâmetro de interesse e $\theta_{1}$ o parâmetro "nuisance", e posteriormente aplicaremos uma reparametrização logarítmica no parâmetro ortogonal obtido.

A ortogonalização em $\theta_{1}$ é caracterizada pela transformação dos parâmetros originais $\theta_{1}$ e $\beta$, nos parâmetros ortogonais $\varphi$ e $\beta$.

Essa reparametrização é obtida resolvendo-se a equação diferencial,

$$
-I_{\theta_{1}, \beta}=I_{\theta_{1}, \theta_{1}} \frac{\partial \theta_{1}}{\partial \beta}
$$

onde $I_{\theta_{1}, \theta_{1}}$ e $I_{\theta_{1}, \beta}$ são os elementos da matriz de informação de Fisher (2.28) com respeito a $\theta_{1}$ e do produto cruzado $\left(\theta_{1}, \beta\right)$, respectivamente. 
Primeiramente podemos observar que a função de verossimilhança para $\boldsymbol{\theta}_{\mathbf{1}}$ e $\beta(2.23)$ pode ser reescrita na forma,

$$
L\left(\theta_{1}, \beta\right) \propto \theta_{1}^{-r} \exp \left\{\beta \sum_{i=1}^{k} r_{i}\left(\frac{1}{V_{1}}-\frac{1}{V_{i}}\right)-\frac{1}{\theta_{1}} \sum_{i=1}^{k} \frac{V_{i}}{V_{1}} A_{i} e^{\beta\left(\frac{1}{V_{1}}-\frac{1}{V_{i}}\right)}\right\}
$$

isto é, definindo

$$
\overline{\bar{V}}=\frac{\sum_{i=1}^{k} r_{i}\left(\frac{1}{V_{1}}-\frac{1}{V_{i}}\right)}{\sum_{i=1}^{k} r_{i}}
$$

como a média ponderada dos $\left(\frac{1}{V_{1}}-\frac{1}{V_{i}}\right)$ 's,

$$
L\left(\theta_{1}, \beta\right) \propto \theta_{1}^{-r} \exp \left\{\beta r \overline{\bar{V}}-\frac{1}{\theta_{1}} \sum_{i=1}^{k} \frac{V_{i}}{V_{1}} A_{i} e^{\beta\left(\frac{1}{V_{1}}-\frac{1}{v_{i}}\right)}\right\} .
$$

Analogamente, reescrevemos os elementos da matriz de informaçäa de Fisher $\left(2.2 \phi^{\prime}\right)$ em função de $\overline{\bar{V}}$,

$$
I\left(\theta_{1}, \beta\right)=\left[\begin{array}{cc}
\frac{r}{\theta_{1}^{2}} & -\frac{r \overline{\bar{V}}}{\theta_{1}} \\
-\frac{r \overline{\bar{V}}}{\theta_{1}} & b_{2}
\end{array}\right] .
$$

Substituindo os elementos de (2.54) na equação diferencial (2.52), ternes,

$$
\overline{\bar{V}} d \beta=\frac{d \theta_{1}}{\theta_{1}} .
$$

Uma solução dessa equação diferencial é dada por $\overline{\bar{V}} \beta=\log \left(\theta_{1}\right)+(a)$, onde $c(\varphi)$ é uma função arbitrária de $\varphi$.

Considerando $c(\varphi)=\log \left(\frac{1}{\varphi}\right)$ obtemos, 


$$
\theta_{1}=\varphi e^{\beta \bar{V}}
$$

Como, de (2.2) e (2.6) sob o nível usual de estresse $V_{1}, \theta_{1}^{-1}=V_{1} \exp \left\{\alpha-\frac{\beta}{V_{1}}\right\}$, temos que,

$$
\varphi=\frac{e^{\beta\left(\frac{1}{\nu_{1}}-\bar{V}\right)}}{V_{1} e^{\alpha}}
$$

e $\alpha$ pode ser escrito na forma,

$$
\alpha=\log \left\{\frac{e^{\left.\mathcal{\beta (} \frac{1}{V_{1}}-\bar{V}\right)}}{V_{1} \varphi}\right\} .
$$

Substituindo a expressão (2.57) na função de verossimilhança (2.7) obtemos a função de verossimilhança para os parâmetros ortogonais, $\varphi$ e $\beta$, dada por,

$$
L(\varphi, \beta) \propto \varphi^{-r} \exp _{1}\left\{-\frac{1}{\varphi} \sum_{i=1}^{k} \frac{V_{i}}{V_{1}} A_{i} e^{\beta\left(\frac{1}{V_{1}}-\frac{1}{V_{i}}-\overline{\bar{V}}\right)}\right\}
$$

Os estimadores de máxima verossimilhança para $\varphi$ e $\beta$ são dados por,



e $\hat{\beta}$ é a solução da equação

$$
\sum_{i=1}^{k} \frac{V_{i}}{V_{1}} A_{i}\left(\frac{1}{V_{1}}-\frac{1}{V_{i}}-\overline{\bar{V}}\right) e^{\hat{\beta}\left(\frac{1}{V_{1}}-\frac{1}{V_{i}}-\overline{\bar{V}}\right)}=0 .
$$

A matriz de informação de Fisher com respeito dos parâmetros $\varphi$ e $\beta$ é dada por, 


$$
I(\varphi, \beta)=\left[\begin{array}{cc}
\frac{r}{\varphi^{2}} & 0 \\
0 & e_{1}
\end{array}\right],
$$

onde $e_{1}=\sum_{i=1}^{k} r_{i}\left(\frac{1}{V_{1}}-\frac{1}{V_{i}}-\overline{\bar{V}}\right)^{2}$.

Como discutido em Cox e Reid (1987), o estimador de $\beta$ obtido em (2.60) é estável com respeito a $\varphi$. Dessa forma, fazendo $\beta=\hat{\beta}$, de (2.55), temos que,

$$
\theta_{1}=\varphi e^{\overline{\bar{V} \hat{\beta}}}
$$

(função um a um de $\varphi$ e $\theta_{1}$ ).

Podemos observar que através da reparametrização ortogonal adotada para o parâmetro $\theta_{1}$ um primeiro passo foi dado com relação à obtenção de uma parametrização assintoticamente independente, isto é, a matriz (2.61) é diagonal, implicando em uma parametrização ortogonal (ver por exemplo, Cox e Reid, 1987). Porém, inferências sobre os parâmetros $\varphi$ e $\beta$ via aproximação Normal assintótica dos estimadores de máxima veróssimilhança não terão boa precisão (os elementos de (2.61) não são constantes). Para solucionar esse problema, consideramos a reparametrização logarítimica, isto é, $\psi=\log \varphi\left(\varphi=e^{\psi}\right)$.

Considerando $\varphi=e^{\psi}$ e a função de verossimilhança (2.58), obtemos a função de yerossimilhança para $\psi$ e $\beta$, dada por,

$$
L(\psi, \beta) \propto e^{-\psi r} \exp \left\{-e^{-\psi} \sum_{i=1}^{k} \frac{V_{i}}{V_{1}} A_{i} e^{\beta\left(\frac{1}{V_{1}}-\frac{1}{V_{i}}-\overline{\bar{V}}\right)}\right\} .
$$

Os estimadores de máxima verossimilhança para $\psi$ e $\beta$ são dados por, 


$$
\hat{\psi}=\log \left\{\frac{\sum_{i=1}^{k} \frac{V_{i}}{V_{1}} A_{i} e^{\hat{\beta}\left(\frac{1}{V_{1}}-\frac{1}{V_{i}}-\overline{\bar{V}}\right)}}{r}\right\}
$$

e $\hat{\beta}$ é a solução da equação

$$
\sum_{i=1}^{k} \frac{V_{i}}{V_{1}} A_{i}\left(\frac{1}{V_{1}}-\frac{1}{V_{i}}-\overline{\bar{V}}\right) e^{\hat{\mathcal{\rho}}\left(\frac{1}{V_{1}}-\frac{1}{V_{i}}-\overline{\bar{V}}\right)}=0
$$

A matriz de informação de Fisher para $\psi$ e $\beta$ é dada por,

$$
I(\psi, \beta)=\left[\begin{array}{ll}
r & 0 \\
0 & e_{1}
\end{array}\right],
$$

onde $e_{1}$ é dado em (2.61).

Como os elementos de $I(\psi, \beta)$ são constantes, uma boa precisão da aproximação Normal assintótica da função de verossimilhança (2.63) está garantida. Além disso, $I(\psi, \beta)$ é uma matriz diagonal o que implica em ortogonalidade entre os parâmetros. Dessa forma, os estimadores de máxima verossimilhança $\hat{\psi}$ e $\hat{\beta}$ são assintoticamente independentes.

Portanto, inferências sobre $\psi$ podem ser baseadas na aproximação Normal assintótica,

$$
\hat{\psi} \stackrel{a}{\sim} N\left(\psi, I^{-1}(\psi)\right)
$$

onde $I^{-1}(\psi)=\frac{1}{r}$ é o elemento da matriz inversa de $I(\psi, \beta)$ com respeito a $\psi$, dada em (2.66). Também como $\varphi=e^{\psi}$, de (2.62), temos, 


$$
\theta_{1}=e^{\psi} e^{\hat{\beta} \bar{V}}
$$

Daí considerando-se o estimador de máxima verossimilhança de $\psi(2.64)$, o estimador de máxima verossimilhança de $\theta_{1}$ é dado por,

$$
\hat{\theta_{1}}=\frac{\sum_{i=1}^{k} \frac{V_{i}}{V_{1}} A_{i} e^{\hat{\beta}\left(\frac{1}{V_{1}}-\frac{1}{V_{i}}\right)}}{r}
$$

onde $\hat{\beta}$ é a solução da equação (2.65). Observe a existência de igualdade entre as expressões (2.25) e (2.69).

\subsubsection{Retrospecto das Parametrizações Adotadas}

Para resumir as parametrizações estudadas neste capítulo construímos o Quadro 2.1 onde podemos obsèvvar que tanto a utilização do método "Delta" quanto o uso da reparametrizaçáo ortogónal não têm efeito no sentido de proporcionar uma aproximação Normal assintótica precisa dos estimadores de máxima verossimilhança, mesmo sabendo que nessas últimas os parâmetros envolvidos são não correlacionados. Já Considerando uma reparametrização logarítimica para o parâmetro $\theta_{1}$ conseguimos obter boa precisão assintótica. Porém, é através da ortogonalização adotada para o parâmetro $\theta_{1}$ e da aplicação de uma reparametrização logarítimica no parâmetro ortogonal obtido que conseguimos uma parametrização assintoticamente independente. 


\begin{tabular}{|c|c|c|c|c|c|}
\hline \multirow[t]{2}{*}{$\begin{array}{l}\text { Parame- } \\
\text { trização } \\
\text { Adotada }\end{array}$} & \multirow{2}{*}{\multicolumn{2}{|c|}{$\begin{array}{l}\text { Matriz de } \\
\text { Informação } \\
\text { de Fisher }\end{array}$}} & \multirow[t]{2}{*}{ Reparametrização } & \multicolumn{2}{|c|}{$\begin{array}{c}\text { Efeito da } \\
\text { Reparametrização } \\
\end{array}$} \\
\hline & & & & Ortogonalid & $\begin{array}{l}\text { Hoa Aprox. } \\
\text { lormal Ausins }\end{array}$ \\
\hline \multirow[t]{2}{*}{$\left(\theta_{1}, \beta\right)$} & \multirow[t]{2}{*}{$I\left(\theta_{1}, \beta\right)=$} & \multirow{2}{*}{$\begin{array}{ll}\frac{r}{\theta_{1}^{2}} & \frac{b_{1}}{\theta_{1}} \\
\frac{b_{1}}{\theta_{1}} & b_{2}\end{array}$} & $\left(\theta_{1}=\frac{e^{\frac{\beta}{V_{1}}}}{V_{1} e^{\alpha}}, \beta\right)^{(*)}$ & \multicolumn{2}{|r|}{ Não } \\
\hline & & & $\begin{array}{l}\left(\theta_{1}=\frac{e^{\beta\left(V_{1}^{-1}-\bar{V}\right.}}{V_{1} e^{\alpha}}, \beta\right)^{(*)} \\
\text { (modificação sugerida } \\
\text { por Mann, Schaffer } \\
\text { e Singpurwalla, 1974) }\end{array}$ & Não & Não \\
\hline $\begin{array}{c}\left(\theta_{1}, \gamma\right) \\
\cdot \\
\end{array}$ & $I\left(\theta_{1}, \gamma\right)=$ & $\begin{array}{cc}\frac{r-b_{1}^{2} / b_{2}}{\theta_{1}^{2}} & 0 \\
0 & b_{2}\end{array}$ & $\begin{array}{c}\left(\theta_{1}, \gamma=\beta+\frac{b_{1}}{b_{2}} \log \theta_{1}\right) \\
\text { (Ortogonalização em } \beta)\end{array}$ & Sim & Não \\
\hline$(\delta, \beta)$ & $I(\delta, \beta)=$ & $\begin{array}{ll}r & b_{1} \\
b_{1} & b_{2}\end{array}$ & $\begin{array}{c}\left(\delta=\log \theta_{1}, \beta\right) \\
\left(\text { Logarítmica em } \theta_{1}\right)\end{array}$ & Não & Sim \\
\hline$(\varphi, \beta)$ & $I=(\varphi, \beta)=$ & $=\left[\begin{array}{ll}\frac{r}{\varphi^{2}} & 0 \\
0 & e_{1}\end{array}\right]$ & $\begin{array}{c}\left(\varphi=\theta_{1} e^{-\beta \overline{\bar{V}}}, \beta\right) \\
\text { (Ortogonalização em } \theta_{1} \text { ) }\end{array}$ & Sim & Não \\
\hline$(\psi, \beta)$ & $I(\psi, \beta)=$ & $\begin{array}{ll}r & 0 \\
0 & e_{1}\end{array}$ & $\begin{array}{c}(\psi=\log \varphi, \beta) \\
(\text { Logaritmica em } \varphi)\end{array}$ & Sim & Sim \\
\hline
\end{tabular}

(") Uso do método "Delta".

Quadro 2.1: Efeito das parametrizaçōes adotadas. 


\section{Capítulo 3}

\section{Uma Análise Bayesiana do Modelo de Eyring com uma Variável Estresse}

Procedimentos alternativos de análise estatística que podem simplificar as interpretações e conclusões de vários problemas práticos consistem dos métodos Bayesianos (ver por exemplo, Box e Tiao, 1973; Press, 1989).

Neste contexto, assumindo dados sob esquema de censuras de tipo II (ver exemplo, Lawless, 1982), uma distribuição Exponencial para os tempos de sobrevivência e uma densidade a priori não-informativa de Jeffreys (ver por exemplo, Box e Tiao, 1973) para os parâmetros do modelo de Eyring com uma variável estresse (1.5), desenvolvemos, neste Capítulo uma análise Bayesiana, usando o método de Laplace para aproximar integrais (ver por exemplo, Tierney e Kadane, 1986; Kass, Tierney e Kadane, 1990) quando não conseguimos achar soluções analíticas explícitas para as densidades a posteriori marginais de interesse. Também estudamos a precisão dos valores aproximados (ver por exemplo, Achcar, 1988 e 1989; Achcar e Smith, 1990d) e exploramos o uso de algumas reparametrizações. 


\subsection{Densidade a Priori Não-Informativa para $\alpha$ e $\beta$}

Para representar o grau de conhecimento sobre os parâmetros $\alpha$ e $\beta$, consideramos a densidade a priori não-informativa de Jeffreys (ver por exemplo, Box e Tiao, 1973), dada por,

$$
\pi(\alpha, \beta) \propto\{\operatorname{det} I(\alpha, \beta)\}^{1 / 2}
$$

onde $I(\alpha, \beta)$ é a matriz de informação de Fisher para $\alpha$ e $\beta$ (2.18).

Observando que, det $I(\alpha, \beta)=r a_{2}-a_{1}^{2}$, temos,

$$
\pi(\alpha, \beta) \propto \text { constante }
$$

onde $-\infty \stackrel{\beta}{i}, \beta<\infty$.

\subsection{Densidade a Posteriori Conjunta para $\alpha$ e $\beta$}

Considerando à priori não-informativa (3.1) e a função de verossimilhança (2.7), a densidade a posterior ́ conjunta para $\alpha$ e $\beta$ é dada por (ver por exemplo, Box e Tiao, 1973),

$$
\pi(\alpha, \beta / \text { dados }) \propto \pi(\alpha, \beta) L(\alpha, \beta)
$$

isto é,

$$
\pi(\alpha, \beta / \text { dados }) \propto \exp \left\{\alpha r-\beta a_{1}-e^{\alpha} \sum_{i=1}^{k} V_{i} A_{i} e^{-\beta / V_{i}}\right\}
$$


onde $-\infty<\alpha, \beta<\infty, r=\sum_{i=1}^{k} r_{i}$ é o número total de falhas observadas e $a_{1}=$ $\sum_{i=1}^{k} r_{i} / V_{i}$.

\subsection{Densidades a Posteriori Marginais para $\alpha$ e $\beta$}

Em Inferência Bayesiana, inferências são tipicamente baseadas nas densidades a posteriori marginais dos parâmetros envolvidos.

Nesta seção explicitamos as densidades para os parâmetros do modelo de Eyring com uma variável estresse (1.5), $\alpha$ e $\beta$.

\subsubsection{Densidade a Posteriori Marginal para $\beta$}

A densidade a posteriori marginal para $\beta$ é obtida integrando-se a densidade a posteriori conjunta (3.2) com respeito a $\alpha$, isto é,

$$
\begin{aligned}
\pi(\beta / \text { dados }) & =\int_{-\infty}^{\infty} \pi(\alpha, \beta / \text { dados }) d \alpha \\
& \propto e^{-\beta a_{1}} \int_{-\infty}^{\infty} e^{\alpha r} \exp \left\{-e^{\alpha} \cdot \sum_{i=1}^{k} V_{i} A_{i} e^{-\beta / V_{i}}\right\} d \alpha
\end{aligned}
$$

Para resolver (3.3), considere a transformação $u=e^{\alpha}$. Como $d \alpha=d u / u$,

$$
\pi(\beta / \text { dados }) \propto e^{-\beta a_{1}} \int_{0}^{\infty} u^{r-1} \exp \left\{-u \sum_{i=1}^{k} V_{i} A_{i} e^{-\beta / V_{i}}\right\} d u
$$

Observando que, 


$$
\int_{0}^{\infty} u^{r-1} \exp \left\{-u \sum_{i=1}^{k} V_{i} A_{i} e^{-\beta / V_{i}}\right\} d u=\frac{\Gamma(r)}{\left\{\sum_{i=1}^{k} V_{i} A_{i} e^{-\beta / V_{i}}\right\}^{r}}
$$

(ver por exemplo, Box e Tiao, 1973), a densidade a posteriori marginal para $\beta$ é dada por,

$$
\pi(\beta / \text { dados }) \propto \frac{e^{-\beta a_{1}}}{\left\{\sum_{i=1}^{k} V_{i} A_{i} e^{-\beta / V_{i}}\right\}^{r}},
$$

onde $-\infty<\beta<\infty$.

\subsubsection{Densidade a Posteriori Marginal para $\alpha$}

Integrando a densidade a posteriori conjunta (3.2) com respeito a $\beta$, obtemos a densidade a posteriori marginal para $\alpha$ dada por,

$$
\pi(\alpha / \text { dados })=\int_{-\infty}^{\infty} \pi(\alpha, \beta / \text { dados }) d \beta,
$$

isto é,

$$
\pi(\alpha / \text { dados }) \propto e^{\alpha r} \int_{-\infty}^{\infty} e^{-n h_{\alpha}(\beta)} d \beta
$$

onde $-n h_{\alpha}(\beta)=-\beta a_{1}-e^{\alpha} \sum_{i=1}^{k} V_{i} A_{i} e^{-\beta / V_{i}}$.

Em geral, os métodos Bayesianos estão sujeitos a algumas limitaçôes relacionadas a resolução de integrais que podem não apresentar soluções analíticas explícitas. Para resolvermos este problema, uma possibilidade é o uso de métodos 
de aprcximação para integrais (ver por exemplo, Tierney e Kadane, 1986).

Como não conseguimos achar uma solução analítica explícita para a densidade a posteriori marginal para $\alpha$ (3.5), exploramos o uso do método de Laplace para aproximação de integrais (ver exemplo, Tierney e Kadane, 1986; Kass, Tierney e Kadane, 1990; Apêndice 2) - um dos métodos mais utilizados em inferência Bayesiana aproximada (ver por exemplo, Achcar, 1988).

A primeira e a segunda derivada de $-n h_{\alpha}(\beta)$ com respeito a $\beta$ são dadas por,

$$
-n h_{\alpha}^{\prime}(\beta)=-a_{1}+e^{\alpha} \sum_{i=1}^{k} A_{i} e^{-\beta / v_{i}}
$$

e

$$
n h_{\alpha}^{\prime \prime}(\beta)=-e^{\alpha} \sum_{i=1}^{k} \frac{A_{i}}{V_{i}} e^{-\beta / V_{i}}
$$

respectivamente.

Usando o método de Laplace completamente exponencial para aproximação de integrais (ver Apêndice 2) obtemos a densidade a posteriori marginal aproximada para $\alpha$ dada por,

$$
\pi(\alpha / d a d o s) \propto \frac{\exp \left\{\alpha(r-1 / 2)-\hat{\beta} a_{1}-e^{\alpha} \sum_{i=1}^{k} V_{i} A_{i} e^{-\hat{\beta} / V_{i}}\right\}}{\left\{\sum_{i=1}^{k} \frac{A_{i}}{V_{i}} e^{-\hat{\beta} / V_{i}}\right\}}
$$

onde $-\infty<\alpha<\infty$ e $\hat{\beta}$ é o valor que maximiza $-n h_{\alpha}(\beta)$, em (3.5), para cada valor fixo de $\alpha$, isto é, $-n h_{\alpha}^{\prime}(\hat{\beta})=0$. 
Ao utilizarmos o método de Laplace para aproximação de integrais, tornase imprescindivel questionarmos a precisão dos valores aproximandos uma vez que esse método é uma aproximação Normal para densidade a posteriori de interesse (ver por exemplo, Tierney e Kadane, 1986). Achcar (1988 e 1989) e Achcar e Smith (1990d) apresentam vários aspectos de inferência Bayesiana aproximada, observando que, em geral, aproximações de Laplace completamente exponenciais para integrais tornam-se mais precisas quando consideramos uma parametrização envolvendo uma priori não-informativa de Jeffreys localmente uniforme (ver por exemplo, Box e Tiao, 1973).

Neste contexto, podemos observar que a priori não-informativa de Jeffreys (3.1) é uma priori localmente uniforme (ver por exemplo, Box e Tiao, 1973), o que implica, em geral, em uma boa precisão da aproximação Normal para a densidade a posteriori marginal para $\alpha(3.6)$.

\subsection{Densidade a Posteriori para o Tempo Médio de Sobrevivência, $\theta_{1}$, sob o nível Usual de Estresse}

Como visto anteriormente, usualmente, o objetivo dos pesquisadores de indústrias de várias áreas é inferir sobre $\theta_{1}=1 / \lambda_{1}$ (tempo médio de sobrevivência do componente manufaturado sob o nivel usual de funcionamento, $V_{1}$ ).

Considerando-se a trasformação de variáveis $\theta_{1}=e^{\beta / V_{1}} / V_{1} e^{\alpha}$ e $\beta=\beta$, isto é, $\alpha=\beta / V_{1}-\log \left(\theta_{1}\right)-\log \left(V_{1}\right)$ e $\beta=\beta$, e a densidade a posteriori conjunta (3.2), a densidade a posteriori conjunta para $\theta_{1}$ e $\beta$ é dada por,

$$
\pi\left(\theta_{1}, \beta / \text { dados }\right) \propto \theta_{1}^{-1}\left(\frac{e^{\beta / V_{1}}}{\theta_{1} V_{1}}\right)^{r} \exp \left\{-\beta a_{1}-\frac{e^{\beta / V_{1}}}{\theta_{1} V_{1}} \sum_{i=1}^{k} V_{i} A_{i} e^{-\beta / V_{i}}\right\}
$$


isto é,

$$
\pi\left(\theta_{1}, \beta / \text { dados }\right) \propto \theta_{1}^{-(r+1)} \exp \left\{-\beta\left(a_{1}-\frac{r}{V_{1}}\right)-\frac{1}{\theta_{1}} \sum_{i=1}^{k} \frac{V_{i}}{V_{1}} A_{i} e^{-\beta\left(\frac{1}{V_{i}}-\frac{1}{V_{1}}\right)}\right\}
$$

onde $\theta_{1}>0,-\infty<\beta<\infty, r=\sum_{i=1}^{k} r_{i}$ e $a_{1}=\sum_{i=1}^{k} r_{i} / V_{i}$.

Integrando a densidade a posteriori conjunta (3.7) com respeito a $\beta$, obtemos a densidade a posteriori marginal para $\theta_{1}$, dada por,

$$
\pi\left(\theta_{1} / d a d o s\right) \propto \theta_{1}^{-(r+1)} \int_{-\infty}^{\infty} e^{-n h_{\theta_{1}}(\beta)} d \beta
$$

$$
\text { onde }-n h_{\theta_{1}}(\beta)=-\beta\left(a_{1}-r / V_{1}\right)-\theta_{1}^{-1} \sum_{i=1}^{k}\left(V_{i} / V_{1}\right) A_{i} e^{-\beta\left(1 / V_{i}-1 / V_{1}\right)} \text {. }
$$

Derivando $-n h_{\theta_{1}}(\beta)$ com respeito a $\beta$ obtemos,

$$
-n h_{\theta_{1}}^{\prime}(\beta)=-\left(a_{1}-\frac{r}{V_{1}}\right)+\frac{1}{\theta_{1}} \sum_{i=1}^{k} \frac{V_{i}}{V_{1}} A_{i}\left(\frac{1}{V_{i}}-\frac{1}{V_{1}}\right) e^{-\beta\left(\frac{1}{V_{i}}-\frac{1}{V_{1}}\right)}
$$

A segunda derivada de $-n h_{\theta_{1}}(\beta)$ com respeito a $\beta$ é dada por,

$$
-n h_{\theta_{1}}^{\prime \prime}(\beta)=-\frac{1}{\theta_{1}} \sum_{i=1}^{k} \frac{V_{i}}{V_{1}} A_{i}\left(\frac{1}{V_{i}}-\frac{1}{V_{1}}\right)^{2} e^{-\beta\left(\frac{1}{V_{i}}-\frac{1}{V_{1}}\right)}
$$

Usando o método de Laplace completamente exponencial para aproximação de integrais obtemos a densidade a posteriori marginal aproximada para $\theta_{1}$, dada por,

$$
\pi\left(\theta_{1} / \text { dados }\right) \propto \frac{\theta_{1}^{-(r+1 / 2)} \exp \left\{-\hat{\beta}\left(a_{1}-\frac{r}{V_{1}}\right)-\frac{1}{\theta_{1}} \sum_{i=1}^{k} \frac{V_{i}}{V_{3}} A_{i} e^{-\hat{\beta}\left(\frac{1}{V_{i}}-\frac{1}{V_{1}}\right)}\right\}}{\left\{\sum_{i=1}^{k} V_{i} A_{i}\left(\frac{1}{V_{i}}-\frac{1}{V_{1}}\right)^{2} \exp \left[-\hat{\beta}\left(\frac{1}{V_{i}}-\frac{1}{V_{1}}\right)\right]\right\}^{1 / 2}}
$$


onde $\theta_{1}>0$ e $\beta$ é o valor que maximiza $-n h_{\theta_{1}}(\beta)$, em (3.8), para cada valor fixo de $\theta_{1}$.

Considerando a matriz de informação de Fisher (2.28), a densidade a priori não-informativa de Jeffreys para $\theta_{1}$ e $\beta$ é dada por (ver por exemplo, Box e Tiao, 1973),

$$
\pi\left(\theta_{1}, \beta\right) \propto\left\{\operatorname{det} I\left(\theta_{1}, \beta\right)\right\}^{1 / 2}
$$

isto é,

$$
\pi\left(\theta_{1}, \beta\right) \propto \frac{1}{\theta_{1}}
$$

onde $\theta_{1}>0$ e $-\infty<\beta<\infty$.

Podemos observar que esta densidade a priori não é localmente uniforme, implicando em uma aproximação de Laplace completamente exponencial, em geral, não muito precisa para a densidade a posteriori marginal para $\theta_{1}$ (3.9) (ver por exemplo, Achcar, 1988 e 1989).

De fato, esta aproximação tem sua precisão comprometida tendo em vista que, em geral, varia para mudanças na parametrização escolhida, e uma boa aproximação envolve o uso de uma parametrização adequada (ver por exemplo, Achcar e Smith, 1990d).

\subsection{Reparametrização logarítmica em $\theta_{1}$}

Pretendendo obter uma aproximação de Laplace completamente exponencial precisa (para termos melhores inferências sobre $\theta_{1}$ ) consideramos a transformação 
de variáveis $\delta=\log \left(\theta_{1}\right)$ e $\beta=\beta$, isto é, $\theta_{1}=e^{\delta}$ e $\beta=\beta$.

A densidade a priori não-informativa de Jeffreys para $\delta$ e $\beta$, considerando-se a matriz de informação de Fisher (2.49), é dada por,

$$
\pi(\delta, \beta) \propto \text { constante }
$$

onde $-\infty<\delta, \beta<\infty$ (localmente uniforme), implicando em uma boa precisão da aproximação de Laplace para a densidade a posteriori de $\delta$ (ver por exemplo, Achcar, 1988 e 1989; Achcar e Smith, 1990d).

A densidade a posteriori conjunta na nova parametrização, $\delta$ e $\beta$, considerando-se a transformação $\theta_{1}=e^{\delta}$ e $\beta=\beta$ e a densidade a posteriori conjunta (3.7), é dada por,

$$
\pi(\delta, \beta / \text { dados }) \propto e^{-\delta r} \exp \left\{-\beta\left(a_{1}-\frac{r}{V_{1}}\right)-e^{-\delta} \sum_{i=1}^{k} \frac{V_{i}^{i}}{V_{1}} A_{i} e^{-\beta\left(\frac{1}{V_{i}}-\frac{1}{V_{1}}\right)}\right\},
$$

onde $-\infty<\delta, \beta<\infty$

Portanto, a densidade a posteriori marginal para $\delta$ é dada por,

$$
\pi(\delta / d a d o s) \propto e^{-\delta \tau} \int_{-\infty}^{\infty} e^{-n h_{\delta}(\beta)} d \beta
$$

onde $-n h_{\delta}(\beta)=-\beta\left(a_{1}-r / V_{1}\right)-e^{-\delta} \sum_{i=1}^{k}\left(V_{i} / V_{1}\right) e^{-\beta\left(1 / V_{i}-1 / V_{1}\right)}$.

Calculando a primeira e a segunda derivada de $-n h_{\delta}(\beta)$ com respeito a $\beta$ e usando o método de Laplace completamente exponencial para aproximação de 
integrais obtemos a densidade a posteriori marginal aproximada para $\delta=\log \left(\theta_{1}\right)$, dada por,

$$
\pi(\delta / \text { dados }) \propto \frac{e^{-\delta(r-1 / 2)} \exp \left\{-\hat{\beta}\left(a_{1}-\frac{r}{V_{1}}\right)-e^{-\delta} \sum_{i=1}^{k} \frac{V_{i}}{V_{1}} A_{i} e^{-\hat{\beta}\left(\frac{1}{V_{i}}-\frac{1}{V_{1}}\right)}\right\}}{\left\{\sum_{i=1}^{k} V_{i} A_{i}\left(\frac{1}{V_{i}}-\frac{1}{V_{1}}\right)^{2} e^{-\hat{\beta}\left(\frac{1}{V_{i}}-\frac{1}{V_{1}}\right)}\right\}^{1 / 2}},
$$

onde $-\infty<\delta<\infty$ e $\hat{\beta}$ é o valor que maximiza $-n h_{\delta}(\beta)$ em (3.13).

Assim, encontramos uma métrica paramétrica que só muda em locação e não em escala (a densidade a priori não-informativa de Jeffreys (3.11) é localmente uniforme, ver por exemplo, Box e Tiao (1973)) condicionando à densidade a posteriori marginal para $\delta=\log \left(\theta_{1}\right)$, dada em (3.14), em geral, uma boa aproximação de Laplace completamente exponencial (ver por exemplo, Achcar, 1988 e a989; Achcar e Smith, 1990d), isto é, podemos observar um aumento na precisão da aproximação usando uma parametrização que implica numa priori não-informativa de Jefreys localmente uniforme.

\subsection{O uso de uma Parametrização Independente}

Como pudemos observar no Capítulo 2, a reparametrização ortogonal (ver por exemplo, Cox e Reid, 1987) adotada para o parâmetro $\theta_{1}$ associada a uma reparametrização logarítmica no parâmetro ortogonal obtido possibilitou a obtenção de uma parametrização assintoticamente independente.

No contexto Bayesiano, pretendemos verificar se a utilização dessas reparametrizações proporciona alguma vantagem computacional e/ou melhoria em termos de aproximação de Laplace na obtenção da densidade a posteriori para o parâmetro de interesse $\theta_{1}$. 
Considerando a matriz de informação de Fisher com respeito a $\psi$ e $\beta(2.66)$, a densidade a priori não-informativa de Jeffreys para $\psi$ e $\beta$ é dada por,

$$
\pi(\psi, \beta) \propto \text { constante }
$$

onde $-\infty<\psi, \beta<\infty$ (localmente uniforme).

A densidade a posteriori conjunta para $\psi$ e $\beta$ considerando-se a densidade priori (3.15) e a função de verossimilhança (2.63) é dada por,

$$
\pi(\psi, \beta / \text { dados }) \propto e^{-\psi r} \exp \left\{-e^{-\psi} \sum_{i=1}^{k} \frac{V_{i}}{V_{1}} A_{i} e^{\beta\left(\frac{1}{V_{1}}-\frac{1}{V_{i}}-\overline{\bar{V}}\right)}\right\}
$$

onde $-\infty<\psi, \beta<\infty$ e $\overline{\bar{V}}=\frac{\sum_{i=1}^{k} r i\left(\frac{1}{V_{1}}-\frac{1}{V_{i}}\right)}{r}$.

A densidade a posteriori marginal para $\beta$ é dada por

$$
\pi(\beta / \text { dados }) \propto \int_{-\infty}^{\infty} e^{-\psi} \exp \left\{e^{-\psi} \sum_{i=1}^{k} \frac{V_{i}}{V_{1}} A_{i} e^{\beta\left(\frac{1}{V_{1}}-\frac{1}{V_{i}}-\overline{\bar{V}}\right)}\right\} d \psi
$$

Considerando-se a transformação $u=e^{\psi}$,

$$
\pi(\beta / \text { dados }) \propto \int_{0}^{\infty} u^{-(r+1)} \exp \left\{-u^{-1} \sum_{i=1}^{k} \frac{V_{i}}{V_{1}} A_{i} e^{\beta\left(\frac{1}{V_{1}}-\frac{1}{V_{i}}-\overline{\bar{V}}\right)}\right\} d u
$$

isto é, analogamente à sub-seção 3.3.1,

$$
\pi(\beta / \text { dados }) \propto\left\{\sum_{i=1}^{k} V_{i} A_{i} e^{\beta\left(\frac{1}{v_{1}}-\frac{1}{v_{i}}-\overline{\bar{V}}\right)}\right\}^{-r},
$$


onde $-\infty<\beta<\infty$.

A densidade a posteriori marginal $\psi$ aproximada pelo método de Laplace completamente exponencial é dada por ,

$$
\pi(\psi / \text { dados }) \propto \frac{e^{-\psi(r-1 / 2)} \exp \left\{-e^{-\psi} \sum_{i=1}^{k} \frac{V_{i}}{V_{1}} A_{i} e^{\hat{\beta}\left(\frac{1}{V_{1}}-\frac{1}{V_{i}}-\bar{V}\right)}\right\}}{\left\{\sum_{i=1}^{k} V_{i} A_{i}\left(\frac{1}{V_{1}}-\frac{1}{V_{i}}-\overline{\bar{V}}\right)^{2} e^{\hat{\beta}\left(\frac{1}{V_{2}}-\frac{1}{V_{i}}-\overline{\bar{V}}\right)}\right\}^{1 / 2}}
$$

onde $-\infty<\psi<\infty$ e $\hat{\beta}$ maximiza $-n h \psi(\beta)=-e^{-\psi} \sum_{i=1}^{k} \frac{V_{i}}{V_{1}} A_{i} e^{\beta\left(\frac{1}{V_{1}}-\frac{1}{V_{i}}-\bar{V}\right)}$

Porém, como a parametrização $(\psi, \beta)$ é ortogonal (ver sub-seção 2.6.4) o estimador de $\beta$ é estável com respeito $\psi$; também, como maximizar (3.18) é equivalente a maximizar $-n h \psi(\beta)$ dado em (3.19), Podemos tomar $\hat{\beta}=\tilde{\beta}^{\prime}$ onde $\tilde{\beta}^{\prime}$ é a moda da densidade a posteriori marginal para $\beta$ (3.18).

Daí,

$$
\pi(\psi / \text { dados }) \propto e^{-\psi(r-1 / 2)} \exp \left\{-e^{-\psi} \sum_{i=1}^{k} \frac{V_{i}}{V_{1}} A_{i} e^{\bar{\beta}^{\prime}\left(\frac{1}{V_{1}}-\frac{1}{V_{i}}-\overline{\bar{V}}\right)}\right\}
$$

onde $-\infty<\psi<\infty$.

Na expressão (2.68), fazendo $\hat{\beta}=\tilde{\beta}^{\prime}$, temos,

$$
\theta_{1}=e^{\psi} e^{\bar{\beta}^{\prime} \overline{\bar{V}}}
$$

e a densidade a posteriori para $\theta_{1}$ é dada por,

$$
\pi\left(\theta_{1} / \text { dados }\right)=c \theta_{1}^{-(r+1 / 2)} \exp \left\{-\theta_{1}^{-1} \sum_{i=1}^{k} \frac{V_{i}}{V_{1}} A_{i} e^{\bar{\beta}^{\prime}\left(\frac{1}{V_{1}}-\frac{1}{V_{i}}\right)}\right\}
$$


onde $c=\frac{\left\{\sum_{i=1}^{k} \frac{v_{i}}{V_{1}} A_{i} e^{\tilde{\beta}^{\prime}\left(\frac{1}{v_{1}}-\hat{V}_{i}\right)}\right\}^{r-1 / 2}}{\Gamma(r-1 / 2)}$ (ver por exemplo, Box e Tiao, 1973).

Isto é,

$$
\begin{gathered}
\pi\left(\theta_{1} / \text { dados }\right)=\frac{\left\{\sum_{i=1}^{k} \frac{V_{i}}{V_{1}} A_{i} e^{\tilde{\beta}^{\prime}\left(\frac{1}{V_{1}}-\frac{1}{V_{i}}\right)}\right\}^{r-1 / 2}}{\Gamma(r-1 / 2)} \theta_{1}^{-(r+1 / 2)} \\
\exp \left\{-\theta_{1}^{-1} \sum_{i=1}^{k} \frac{V_{i}}{V_{1}} A_{i} e^{\tilde{\beta}^{\prime}\left(\frac{1}{V_{1}}-\frac{1}{V_{i}}\right)}\right\}
\end{gathered}
$$

onde $\theta_{1}>0$ e $\tilde{\beta}^{\prime}$ é a moda da densidade a posteriori (3.18) (observe que, (3.23) é uma densidade Gama), cuja moda a posteriori é dada por,

$$
\tilde{\theta}_{1}^{\prime}=\frac{\sum_{i=1}^{k} \frac{V_{i}}{V_{1}} A_{i} e^{\bar{\beta}^{\prime}\left(\frac{1}{V_{1}}-\frac{1}{V_{i}}\right)}}{r+1 / 2}
$$

Podemos observar que importantes vantagens foram obtidas através das reparametrizações utilizadas, ou' seja, obtemos boa aproximação de Laplace para a densidade a posteriori marginal de $\psi$ (3.20) e facilidade computacional em seu cálculo, além disso, temos uma expressão sob "forma fechada" para moda a posteriori do parâmetro $\theta_{1}$, atribuída à ortogonalidade entre os parâmetros $\psi$ e $\beta$.

Também, observar que (de (3.23)) a densidade a posteriori para

$$
\frac{2\left\{\sum_{i=1}^{k} \frac{V_{i}}{V_{1}} A_{i} e^{\dot{\beta}^{\prime}\left(\frac{1}{V_{1}}-\frac{1}{V_{i}}\right)}\right\}}{\theta_{1}}
$$

é a -densidade de uma distribuição Qui-Quadrado com $2 r-1$ graus de liberdade. Daí, um intervalo "a posteriori de maior probabilidade" (HPD) $100\left(1-\gamma^{*}\right) \%$ para o tempo médio de sobrevivência $\theta_{1}$ é dado por, 


$$
\left(\frac{2 \sum_{i=1}^{k} \frac{V_{i}}{V_{1}} A_{i} e^{\hat{\beta}^{\prime}\left(\frac{1}{V_{1}}-\frac{1}{V_{i}}\right)}}{\chi_{2 r-1\left(1-\frac{\gamma^{\prime}}{2}\right)}^{2}} ; \frac{2 \sum_{i=1}^{k} \frac{V_{i}}{V_{1}} A_{i} e^{\hat{\beta}^{\prime}\left(\frac{1}{V_{1}}-\frac{1}{V_{i}}\right)}}{\left.\chi_{2 r-1\left(\frac{\gamma^{\prime}}{2}\right)}^{2}\right)}\right) .
$$

onde $\tilde{\beta}^{\prime}$ é a moda da densidade a posteriori marginal para $\beta(3.18)$ e $\chi_{2 r-1\left(\frac{x^{+}}{2}\right)}^{2}$ é o quantil da distribuição Qui-Quadrado dado por,

$$
P\left(\chi_{2 r-1}^{2} \leq \chi_{2 r-1\left(\frac{\gamma^{\prime}}{2}\right)}^{2}\right)=\frac{\gamma^{\prime}}{2}
$$

Observe que, apesar de termos considerado até o momento, somente o uso da reparametrização logarítmica e da reparametrização assintoticamente independente na análise Bayesiana do modelo, poderíamos considerar quaisquer das reparametrizações estudadas no Capítulo 2, sendo que as densidades a posteriori para os parâmetros envolvidos em cada uma delas podem ser obtidas facilmente de maneira análoga à descrita neste capítulo. Também, observe que uma expressão equivalente à (3.23) poderia ser obtida utilizando-se a parametrizaçẩ $(\varphi ; \beta)$ (ver sub-seção 2.6.4). 


\section{Capítulo 4}

\section{Controle de Qualidade em testes de Sobrevivência Acelerados}

No controle de qualidade de produtos industriais torna-se imprescindível a obtenção permanente de medidas da confiabilidade do produto produzido. Em geral, esse controle é feito em meio a linha de produção, selecionando-se uma amostra aleatória para teste, e um resultado rápido, com custo minímo, se faz necessário.

Neste capitulo, determinamos um procedimento de controle de qualidade que pode ser aplicado rapidamente, com custo reduzido, usando testes de sobrevivência acelerados e considerando a densidade preditiva de uma observação futura (ver por exemplo, Aitchinson e Dunsmore, 1975; Press, 1989). Assim, propomos um critério para achar o tempo requerido de teste em um dado nível de estresse, ou obter o nível de estresse necessário para um determinado tempo experimentação fixo. 


\section{4.i Densidade Preditiva para uma Observação Futura em um Nível de estresse $V_{j} \operatorname{com} \beta$ conhecido}

Assumindo $\beta$ conhecido, de (2.7), obtemos a função de verosimilhança para $\alpha$, dada por,

$$
L(\alpha) \propto \exp \left\{\alpha r-e^{\alpha} \sum_{i=1}^{k} V_{i} A_{i} e^{-\beta / V_{i}}\right\}
$$

onde $r=\sum_{i=1}^{k} r_{i}$ é o número de falhas observadas em um teste de sobrevivência acelerado com $k$ níveis aleatorizados da variável estresse temperatura.

A informação de Fisher para $\alpha$, considerando-se a matriz (2.18), é dada por,

$$
I(\alpha)=r
$$

Daí, a densidade a priori não-informativa de Jeffreys para $\alpha$ é dada por,

$$
\pi(\alpha) \propto \text { constante }
$$

onde $-\infty<\alpha<\infty$ (localmente uniforme).

Portanto, de (4.1) e (4.3), a densidade a posteriori para $\alpha$ é dada por,

$$
\pi(\alpha / \text { dados })=c \exp \left\{\alpha r-e^{\alpha} \sum_{i=1}^{k} V_{i} A_{i} e^{-\beta / V_{i}}\right\}
$$

onde $c^{-1}=\int_{-\infty}^{\infty} e^{\alpha r} \exp \left\{-e^{\alpha} \sum_{i=1}^{k} V_{i} A_{i} e^{-\beta / V_{i}}\right\} d \alpha$. 
Considerando a transformação de variáveis, $u=e^{\alpha}$, temos, $c^{-1}=\int_{0}^{\infty} u^{r-1} e^{-u a} d u$, onde $a=\sum_{i=1}^{k} V_{i} A_{i} e^{-\beta / V_{i}}$, isto é, $c^{-1}=\Gamma(r) / a^{r}$ (ver por exemplo, Box e Tiao, 1973).

Assim, a densidade a posteriori para $\alpha$ é dada por,

$$
\pi(\alpha / \text { dados })=\frac{\left\{\sum_{i=1}^{k} V_{i} A_{i} e^{-\beta / V_{i}}\right\}^{r}}{\Gamma(r)} \exp \left\{\alpha r-e^{\alpha} \sum_{i=1}^{k} V_{i} A_{i} e^{-\beta / V_{i}}\right\}
$$

onde $-\infty<\alpha<\infty$

A densidade preditiva da observação futura $T_{(n+1) j}$ (onde $n=\sum_{i=1}^{k} n_{i}$ é o número total de observações em teste), considerando um nivel de estresse $V_{j}$ e a densidade a posteriori (4.5) é dada por (ver por exemplo, Aitchinson e Dunsmore, 1975; Press, 1989),

$$
\begin{aligned}
f^{j}\left(t_{(n+1) j} / \text { dados }\right) & =\int_{-\infty}^{\infty} f^{j}\left(t_{(n+1) j} / \alpha\right) \pi(\alpha / \text { dados }) d \alpha \\
& =E_{\alpha / \text { dados }}\left\{f^{j}\left(t_{(n+1) j} / \text { dados }\right)\right\}
\end{aligned}
$$

onde $f^{j}\left(t_{(n+1) j} / \alpha\right)=V_{j} e^{\alpha-\beta / V_{j}} \exp \left\{-V_{j} e^{\alpha-\beta / V_{j}} t_{(n+1) j}\right\}$, isto é,

$$
\begin{aligned}
f^{j}\left(t_{(n+1) j} / \text { dados }\right)= & V_{j} e^{-\beta / V_{j}} \frac{\left\{\sum_{i=1}^{k} V_{i} A_{i} e^{-\beta / V_{i}}\right\}^{r}}{\Gamma(r)} . \\
& \cdot \int_{-\infty}^{\infty} \exp \left\{\alpha(r+1)-e^{\alpha}\left[V_{j} e^{-\beta / V_{j}} t_{(n+1) j}+\sum_{i=1}^{k} V_{i} A_{j} E^{-\beta / V_{j}}\right]\right\} d \alpha .
\end{aligned}
$$

Considerando novamente a transformação de variáveis, $u=e^{\alpha}$, obtemos, 


$$
f^{j}\left(t_{(n+1) j} / \text { dados }\right)=V_{j} e^{-\beta / V_{j}} \frac{\left\{\sum_{i=1}^{k} V_{i} A_{i} e^{-\beta / V_{i}}\right\}^{r}}{\Gamma(r)} \cdot \int_{0}^{\infty} u^{r} e^{-u b} d u,
$$

onde $b=V_{j} e^{-\beta / V_{j}} t_{(n+1) j}+\sum_{i=1}^{k} V_{i} A_{i} e^{-\beta / V_{i}}$

Observando que $\int_{0}^{\infty} u^{r} e^{-u b} d u=\Gamma(r+1) / b^{r+1}$ (ver por exemplo, Box e Tiao, 1973), e que $\Gamma(r+1)=r \Gamma(r)$,

$$
f^{j}\left(t_{(n+1) j} / \text { dados }\right)=\frac{r V_{j} e^{-\beta / V_{j}}\left\{\sum_{i=1}^{k} V_{i} A_{i} e^{-\beta / V_{i}}\right\}^{r}}{\left\{V_{j} e^{-\beta / V_{j}} t_{(n+1) j}+\sum_{i=1}^{k} V_{i} A_{i} e^{-\beta / V_{i}}\right\}^{r+1}},
$$

onde $t_{(n+1) j}>0$ (densidade de Pareto).

\subsection{Densidade Preditiva para uma Observação Futura em um Nível de Estresse $V_{j}$ assu- mindo $\beta$ desconhecido}

Considere a função de verossimilhança para $\alpha$ e $\beta$ dada (em (2.41)) por

$$
L(\alpha, \beta) \propto \exp \left\{\alpha r-e^{\alpha} \sum_{i=1}^{k} V_{i} A_{i} e^{-\beta\left(V_{i}^{-1}-\bar{V}\right)}\right\}
$$

onde $\bar{V}=\frac{\sum_{i=1}^{k} r_{1} / V_{i}}{\sum_{i=1}^{k} r_{i}}$

A priori não informativa de Jeffreys para $\alpha$ e $\beta$ é dada (de (2.44)) por,

$$
\pi(\alpha, \beta) \propto \text { constante, }
$$

onde $-\infty<\alpha, \beta<\infty$. 
A densidade a posteriori para $\alpha$ e $\beta$ é dada (de (4.10)) por,

$$
\pi(\alpha, \beta / d a d o s) \propto \exp \left\{\alpha r-e^{\alpha} \sum_{i=1}^{k} V_{i} A_{i} e^{-\beta\left(V_{i}^{-1}-\bar{V}\right)}\right\}
$$

onde $-\infty<\alpha, \beta<\infty$

A densidade preditiva da observação futura $T_{(n+1) j}$ sob o nível de estresse $V_{j}$ é dada por,

$$
\left.f^{j}\left(t_{(n+1) j} / d a d o s\right)=\int_{-\infty}^{\infty} \int_{-\infty}^{\infty} f^{j}\left(t_{(n+1) j} / \alpha, \beta\right) \pi(\alpha, \beta) / d a d o s\right) d \alpha d \beta
$$

onde $f^{j}\left(t_{(n+1) j} / \alpha, \beta\right)=V_{j} e^{\alpha-\beta\left(V_{i}^{-1}-\bar{V}\right)} \exp \left\{-V_{j} e^{\alpha-\beta\left(V_{i}^{-1}-\bar{V}\right)} t_{(n+1) j}\right\}$ e $\pi(\alpha, \beta /$ dados $)$ é a densidade posteriori dada em (4.12).

Definindo $W_{i}=V_{i}^{-1}-\bar{V}, i=1,2, \ldots, k$, temos,

$$
f^{j}\left(t_{(n+1) j} / d a d o s\right) \propto \int_{-\infty}^{\infty} e^{-\beta W_{j}}\left[\int_{-\infty}^{\infty} \exp \left\{\alpha(r+1)-e^{\alpha} c(\beta)\right\} d \alpha\right] d \beta
$$

onde $c(\beta)=V_{j} e^{-\beta W_{j}} t_{(n+1) j}+\sum_{i=1}^{k} V_{i} A_{i} e^{-\beta W_{i}}$.

Fazendo a transformação $u=e^{\alpha}$, temos que

$$
\int_{-\infty}^{\infty} e^{\alpha(r+1)} \exp \left\{-e^{\alpha} c(\beta)\right\} d \alpha=\int_{0}^{\infty} u^{r} e^{-u c(\beta)} d u=\frac{\Gamma(r+1)}{\{c(\beta)\}^{r+1}}
$$

(ver por exemplo, Box e Tiao, 1973).

Portanto, 


$$
f^{j}\left(t_{(n+1) j} / d a d o s\right) \propto \int_{-\infty}^{\infty} e^{-n h(\beta)} d \beta
$$

onde $n h(\beta)=\beta W_{j}+(r+1) \log (c(\beta))$.

Usando o método de Laplace completamente exponencial, temos que,

$$
f^{j}\left(t_{(n+1) j} / \text { dados }\right) \propto \frac{e^{-\hat{\beta} W_{j}}[c(\hat{\beta})]^{-(r+1 / 2)}}{\left\{(r+1)^{2} c^{\prime \prime}(\hat{\beta})-W_{j}^{2} c(\hat{\beta})\right\}^{1 / 2}}
$$

onde $t_{(n+1) j}>0, \hat{\beta}$ é a solução da equação $\frac{c^{\prime}(\hat{\beta})}{c(\hat{\beta})}=-\frac{W_{j}}{(r+1)}, c^{\prime \prime}(\beta)=V_{j} W_{j}^{2} e^{-\beta W_{j}}+$ $\sum_{i=1}^{k} V_{i} A_{i} W_{i}^{2} e^{-\beta W_{j}}$ e $c(\beta)$ é dada em (4.14).

\subsection{O Uso da Densidade Preditiva $f^{j}\left(t_{(n+1) j} /\right.$ Dados $)$ em Controle de Qualidade}

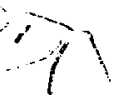

Em controle de qualidade, um procedimento de interesse é selecionar uma amostra aleatória dos componentes de um determinado lote para verificar se estes estão sob controle, isto é, verificar se a confiabilidade dos produtos deste lote está garantida. Porém, isso deve ser feito com tempo e custo de experimentação reduzidos. Assim, consideramos um teste de sobrevivência acelerado com um nível alto de estresse, $V_{j}$, e um período de tempo de teste fixo, $L_{j}$.

Neste procedimento de controle, usualmente, os principais objetivos consistem em determinar:

i)o valor de $L_{j}$ para um dado nível de stress $V_{j}$ fixo (estipulado pelos pesquisadores industriais);

ii)o valor de $V_{j}$, considerando $L_{j}$ fixo. 
Isto é, usando a densidade preditiva (4.9) $\operatorname{com} \beta$ conhecido e considerando uma probabilidade fixa $\left(1-\gamma^{\prime}\right)$, podemos achar os valores requeridos de $V_{j}$ e $L_{j}$, tal que,

$$
P^{j}\left(T_{(n+1) j}>L_{j} / \text { dados }\right)=1-\gamma^{\prime}
$$

Assumindo $\beta$ conhecido, da densidade preditiva (4.9) temos,

$$
P^{j}\left(T_{(n+1) j}>L_{j} / \text { dados }\right)=\int_{L_{j}}^{\infty} f^{j}\left(t_{(n+1) j} / \text { dados }\right) d t_{(n+1) j}
$$

isto é,

$$
P^{j}\left(T_{(n+1) j}>L_{j} / \text { dados }\right)=r\left(\frac{c}{V_{j} e^{-\beta / V_{j}}}\right)^{r} \int_{L_{j}}^{\infty} \frac{d t_{(n+1) j}}{\left\{t_{(n+1) j}+\frac{c}{V_{j} e^{-\beta / V_{j}}}\right\}^{r+1}},
$$

onde $c=\sum_{i=1}^{k} V_{i} A_{i} e^{-\beta p}$

Considerando $P^{j}\left(T_{(n+1) j}>L_{j} /\right.$ dados $)=1-\gamma^{\prime}$, temos,

$$
\frac{\left\{\frac{c}{V_{j} e^{-\beta / V_{j}}}\right\}^{r}}{\left\{L_{j}+\frac{c}{V_{j} e^{-\beta / V_{j}}}\right\}^{r}}=1-\gamma^{\prime} .
$$

Portanto, de (4.19), dado $V_{j}$ (fixo), achamos $L_{j}$ dado por,

$$
L_{j}=\frac{c\left[1-\left(1-\gamma^{\prime}\right)^{1 / r}\right]}{\left(1-\gamma^{\prime}\right)^{1 / r} V_{j} e^{-\beta / V_{j}}}
$$

e com $L_{j}$ fixo, $V_{j}$ é a solução da equação

$$
\frac{c\left[1-\left(1-\gamma^{\prime}\right)^{1 / r}\right]}{L_{j}\left(1-\gamma^{\prime}\right)^{1 / r}}=V_{j} e^{-\beta / V_{j}}
$$


Encontrados os valores de $V_{j}$ e $L_{j}$ tais que (4.17) seja satisfeita, e definindo $p^{j}=P^{j}\left(T_{(n+1) j} \leq L_{j} /\right.$ dados $)$, pode-se colocar $m$ unidades novas em teste sob o nível de estresse, $V_{j}$, e observar seus tempos de sobrevivência durante um período fixo de tempo, $L_{j}$. Denotando por $\mathrm{X}$ o número de falhas em $L_{j}$, e assumindo $X \sim \operatorname{bin}\left(m, p^{j}\right)$ (distribuição binomial), o lote de componentes está sob controle se não rejeitamos a hipótese $H_{o}: p^{j} \leq \gamma^{\prime}$.

Se $m$ é grande $(m \geq 30)$ então temos, $X \stackrel{a}{\sim} N\left\{m p^{j} ; m p^{j}\left(1-p^{j}\right)\right\}$, e um critério de controle de qualidade pode ser baseado no teste de hipótese usual, isto é, o lote está fora de controle se $Z \geq z_{\alpha^{\prime}}$, onde $\alpha^{\prime}$ é um nível de significância, $Z=\left(X-m \gamma^{\prime}\right) / \sqrt{m \gamma^{\prime}\left(1-\gamma^{\prime}\right)} \stackrel{a}{\sim} N(0,1)$ e $z_{\alpha^{\prime}}$ é tal que $P\left(Z \geq z_{\alpha^{\prime}}\right)=\alpha^{\prime}$.

Com $\beta$ desconhecido, usando métodos numéricos, podemos achar $V_{j}$ e $L_{j}$ tais que $P\left(T_{(n+1) j}>L_{j} /\right.$ dados $)=1-\gamma^{\prime}$, a partir da densidade preditiva (4.16).

Dessa forma, com o método proposto neste capítulo, os pesquisadores industriais têm liberdade para decidir por qual estratégia adotar em cada caso particular, escolhendo apropriadamente $1-\gamma^{\prime}, V_{j}$ e $L_{j}$ para cada problema prático. 


\section{Capítulo 5}

\section{Análise do Modelo de Eyring Assumindo uma Distribuição de Weibull para os tempos de Sobrevivência}

Em aplicações práticas de testes de sobrevivência acelerados, dificilmente existem situações onde a distribuição dos tempos de sobrevivência é Exponencial. Uma distribuição muito utilizada é a de Weibull, dada a sua grande flexibilidade.

Assumindo uma distribuição de Weibull para os tempos de sobrevivência e um mecanismo de censura de tipo II, desenvolvemos neste capítulo uma análise Bayesiana do modelo de Eyring com uma variável estresse usando a regra multiparamétrica de Jeffreys (ver por exemplo, Box e Tiao, 1973) para achar uma densidade a priori não-informativa para os parâmetros envolvidos.

\subsection{Formulação do Modelo}

Considere a variável aleatória $T^{*}$ denotando o tempo de sobrevivência de uma unidade com uma densidade de Weibull dada por, 


$$
f\left(t^{*} ; \lambda^{*}, p\right)=\lambda^{*} p\left(\lambda^{*} t^{*}\right)^{p-1} \exp \left\{-\left(\lambda^{*} t^{*}\right)^{p}\right\}
$$

onde $t^{*}>0, \lambda^{*}, p>0$; e assuma que a variável estresse $V$ influencia o parâmetro de escala $\lambda^{*}$, e que o parâmentro de forma $p$ é comum para todos os níveis de estresse.

Assumindo $k$ níveis aleatorizados de estresse e um mecanismo de censura de tipo II para os dados, colocamos $n_{i}$ unidades em teste no j-ésimo nível de estresse, $V_{i}$, e o experimento termina quando $r_{i}$ falhas são observadas. Dessa forma, temos $r_{i}$ observações ordenadas não-censuradas dadas por, $t_{i 1}^{*}, t_{i 2}^{*}, \ldots, t_{i_{r_{i}}}^{*}$ e $n_{i}-r_{i}$ observações iguais a $t_{i_{i}}^{*}, i=1,2, \ldots, k$.

No i-ésimo nivel de estresse $V_{i}$ a função de verossimilhança para $\lambda_{i}^{*}$ e $p$ é dada por,

$$
L_{i}\left(\lambda_{i}^{*}, p\right)=\prod_{j=1}^{r_{i}} f\left(t_{i j}^{*} ; \lambda_{i}^{*}, p\right) S^{n_{i}-r_{i}}\left(t_{i r_{i}}^{*} ; \lambda_{i}^{*} ; p\right)
$$

onde $S\left(t_{i j}^{*} ; \lambda_{i}^{*}, p\right)=e^{-\left(\lambda_{i}^{*} t_{i r_{i}}^{*}\right)^{p}}$ é a função de confiabilidade e $f\left(t_{i j}^{*} ; \lambda_{i}^{*}, p\right)$ é a função densidade de probabilidade (5.1).

$$
\begin{aligned}
\text { Definindo } A_{i}(p) & =\sum_{j=1}^{r_{i}} t_{i j}^{* p}+\left(n i-r_{i}\right) t_{i r_{i}}^{* p}, \text { temos, } \\
L_{i}\left(\lambda_{i}^{*}, p\right) & =p^{r_{i}} \lambda_{i}^{* p r_{i}}\left(\prod_{j=1}^{r_{i}} t_{i j}^{* p-1}\right) \exp \left\{-\lambda_{i}^{* p} A_{i}(p)\right\} .
\end{aligned}
$$

Considerando dados de $k$ níveis aleatorizados de estresse, $V_{1}, V_{2}, \ldots V_{k}, \mathrm{e}$ a relação estresse resposta representada pelo modelo de Eyring com uma variável estresse,

$$
\lambda_{i}^{*}=V_{i} \exp \left\{\alpha-\beta / V_{i}\right\}
$$


a função de verossimilhança para os parâmetros $\alpha, \beta$ e $p$ é dada por,

$$
\begin{aligned}
& L(\alpha, \beta, p)=p^{r}\left(\prod_{i=1}^{k} \prod_{j=1}^{r_{i}} t_{i j}^{p-1}\right)\left(\prod_{i=1}^{k} V_{i}^{p r_{i}}\right) \\
& \exp \left\{p \alpha r-p \beta a_{1}-e^{p \alpha} \sum_{i=1}^{k} V_{i}^{p} A_{i}(p) e^{-\beta p / V_{i}}\right\}
\end{aligned}
$$

onde $r=\sum_{i=1}^{k} r_{i}, a_{1}=\sum_{i=1}^{k} r_{i} / V_{i}$ e $A_{i}(p)=\sum_{j=1}^{r_{i}} t_{i j}^{*^{p}}+\left(n i-r_{i}\right) t_{i i_{i}}^{*^{p}}$ (observar que com $p=1$ temos (5.5) igual à expressão (2.7)).

\subsection{Análise Bayesiana do Modelo Assumindo $\alpha, \beta$ e $p$ desconhecidos}

A densidade a priori para os parânientros $\alpha, \beta$ e $p$ pode ser escrita na forma,

$$
\pi(\alpha, \beta, p)=\pi(\alpha, \beta / p) \pi_{0}(p) .
$$

Usando a regra multiparamétrica de Jeffreys temos (ver por exemplo, Box e Tiao, 1973),

$$
\pi(\alpha, \beta / p) \propto\left\{\operatorname{det} I_{p}(\alpha, \beta)\right\}^{1 / 2}
$$

onde $I_{p}(\alpha, \beta)$ é a matriz de informação de Fisher com respeito a $\alpha$ e $\beta$ dado o parâmetro de forma $p$.

A função log-verossimilhança para $\alpha, \beta$ e $p(\operatorname{de}(5.5))$ é dada por, 


$$
\left.\begin{array}{c}
\ell(\alpha, \beta, p) \propto r \log (p)+(p-1) \sum_{i=1}^{k} \sum_{j=1}^{r_{i}} \log \left(t_{i j}^{*}\right)+p \sum_{i=1}^{k} r_{i} \log \left(V_{i}\right)+ \\
+p \alpha r-p \beta a_{1}-e^{p \alpha} \sum_{i=1}^{k} V_{i}^{p} A_{i}(p) e^{-\beta p / V_{i}} .
\end{array}\right\}
$$

As segundas derivadas de $\ell(\alpha, \beta, p)$ para um dado valor de $p$ são dadas por,

$$
\begin{aligned}
& \frac{\partial^{2} \ell}{\partial \alpha^{2}}=-p^{2} e^{p \alpha} \sum_{i=1}^{k} V_{i}^{p} A_{i}(p) e^{-\beta p / V_{i}} \\
& \frac{\partial^{2} \ell}{\partial \beta^{2}}=-p^{2} e^{p \alpha} \sum_{i=1}^{k} V_{i}^{p-2} A_{i}(p) e^{-\beta p / V_{i}} \\
& \frac{\partial^{2} \ell}{\partial \alpha \partial \beta}=p^{2} e^{p \alpha} \sum_{i=1}^{k} V_{i}^{p-1} A_{i}(p) e^{-\beta p / V_{i}}
\end{aligned}
$$

Como a variável aleatória $T=T^{* p}$ tem uma distribuição Exponencial com parâmetro $\lambda=\lambda^{* p}$ (ver por exemplo, Lawless, 1982), $E\left[A_{i}(p)\right]=\frac{r_{i}}{\lambda_{i}^{* p}}, \lambda_{i}^{*}=$ $V_{i} \exp \left\{\alpha-\beta / V_{i}\right\}, i=1,2, \ldots, k$, isto é ,

$$
E\left[A_{i}(p)\right]=\frac{r_{i}}{V_{i}^{p} \exp \left\{p\left(\alpha-\beta / V_{i}\right)\right\}}
$$

Portanto, a matriz de informação de Fisher para $\alpha$ e $\beta$ dado $p$ é dada por,

$$
I_{p}(\alpha, \beta)=\left[\begin{array}{ll}
r p^{2} & -a_{1} p^{2} \\
-a_{1} p^{2} & a_{2} p^{2}
\end{array}\right]
$$

onde $r$ e $a_{1}$ são dados em (5.5) e $a_{2}=\sum_{i=1}^{k} r_{i} / V_{i}^{2}$ (observar que com $p=1$ a matriz (5.9) iguala-se a matriz (2.18)).

Assim,

$$
\left.\operatorname{det}\left\{I_{p}(\alpha, \beta)\right\}=p^{4}\left(r a_{2}-a_{1}^{2}\right), \quad \text { e de }(5.7)\right)
$$




$$
\pi(\alpha, \beta / p) \propto p^{2}
$$

onde $p>0$ e $-\infty<\alpha, \beta<\infty$.

Assumindo uma densidade a priori localmente uniforme para $\log (p)$ isto é,

$$
\pi_{0}(p) \propto \frac{1}{p}
$$

onde $p>0$, a densidade a priori não-informativa para $\alpha, \beta$ e $p$ baseada na regra multiparamétrica de Jeffreys (ver(5.7)) é dada por,

$$
\pi(\alpha, \beta, p) \propto p
$$

onde $p>0$ e $-\infty<\alpha, \beta<\infty$.

\subsubsection{Densidade a Posteriori Marginal para $\mathrm{p}$}

Considerando a densidade a priori (5.12) e a função de verossimilhança (5.5), a densidade a posteriori conjunta para $\alpha, \beta$ e $p$ é dada por,

$$
\begin{aligned}
\pi(\alpha, \beta, p / \text { dados }) \propto & p^{r+1}\left(\prod_{i=1}^{k} \prod_{j=1}^{r_{i}} t_{i j}^{*^{p-1}}\right)\left(\prod_{i=1}^{k} V_{i}^{p r_{i}}\right) \\
& \cdot \exp \left\{p \alpha r-p \beta a_{1}-e^{p \alpha} \sum_{i=1}^{k} V_{i}^{p} A_{i}(p) e^{-\beta p / V_{i}}\right\}
\end{aligned}
$$

onde $p>0$ e $-\infty<\alpha, \beta<\infty$ (Se $p=1$ temos (5.13) igual a densidade a posteriori conjunta para $\alpha$ e $\beta(3.2)$ ).

A densidade a posteriori marginal para $p$ é dada (de (5.13)) por, 


$$
\begin{gathered}
\pi(p / \text { dados }) \propto p^{r+1}\left(\prod_{i=1}^{k} \prod_{j=1}^{r_{i}} t_{i j}^{*^{p-1}}\right)\left(\prod_{i=1}^{k} V_{i}^{p r_{i}}\right) \int_{-\infty}^{\infty} \int_{-\infty}^{\infty} e^{-n h_{p}(\alpha, \beta)} d \alpha d \beta \\
\text { onde }-n h_{p}(\alpha, \beta)=p \alpha r-p \beta a_{1}-e^{p \alpha} \sum_{i=1}^{k} V_{i}^{p} A_{i}(p) e^{-\beta p / V_{i}}
\end{gathered}
$$

Usando o método de Laplace completamente exponencial para aproximação de integrais (ver por exemplo, Tierney e Kadane, 1986; Apêndice 2) achamos a densidade a posteriori marginal aproximada para $p$ dada (ver Apêndice 2.1) por,

$$
\pi(p / \text { dados }) \propto \frac{p^{r-1} e^{-p a_{1} \dot{\beta}}\left(\prod_{i=1}^{k} \prod_{j=1}^{r_{i}} t_{i j}^{*^{p-1}}\right)\left(\prod_{i=1}^{k} V_{i}^{p r_{i}}\right)}{\left\{r^{2} \sum_{i=1}^{k} V_{i}^{p-2} A_{i}(p) e^{-\hat{\beta} p / V_{i}}-a_{1}^{2}\right\}^{1 / 2}\left\{\sum_{i=1}^{k} V_{i}^{p} A_{i}(p) e^{-\hat{\beta} p / V_{i}}\right\}^{r}}
$$

onde $p>0$ e $\hat{\beta}$ maximiza $g(\beta)=r \log \left\{\frac{r}{\sum_{i=1}^{k} V_{i}^{p} A_{i}(p) e^{-\hat{\beta}_{p} / V_{i}}}\right\}-p \beta a_{1}-r$ para cada valor fixo de $p$.

Portanto, considerando $T=T^{*^{p}}$ com uma distribuição Exponencial com parâmetro $\lambda=\lambda^{*^{p}}$ com o valor de $p$ dado pela moda da densidade a posteriori marginal para $p$ (5.15), podemos considerar uma análise Bayesiana padrão do modelo de Eyring (1.5) com uma distribuição Exponencial para os tempos de sobrevivência, como dada nos Capítulos 3 e 4.

Ocorre que, para mantermos a equivalência entre os parâmetros da distribuição de Weibull e da distribuição Exponencial, devemos considerar uma transformação de escala, isto é, $\lambda_{i}^{*}=V_{i} \exp \left\{\alpha-\frac{\beta}{V_{i}}\right\}$, temos que $\lambda_{i}=V_{i}^{p} \exp \left\{p\left(\alpha-\frac{\beta}{V_{i}}\right)\right\}$. Também, $\theta_{1}=\frac{1}{\lambda_{1}}$ é o tempo médio de sobrevivência sob o nível usual de estresse considerando-se os dados transformados $T^{* p}$ com uma uma distribuição Exponencial com parâmetro $\lambda_{1}$.

Daí, na escala original dos dados (onde temos dados com uma distribuição 
de Weibull com parâmetro de escala $\lambda_{1}^{*}=\lambda_{1}^{\frac{1}{p}}$ ) o tempo médio de sobreviência sob o nível de estresse é dado por, $\theta_{1}^{*}=\Gamma\left(1+\frac{1}{p}\right) / \lambda_{1}^{*}$.

Por exemplo, considerando $p=\tilde{p}$ ( $\tilde{p}$ é a moda da densidade a posteriori (5.15)), $\lambda_{i}=V_{i}^{\tilde{p}} \exp \left\{\tilde{p}\left(\alpha-\frac{\beta}{V_{i}}\right)\right\}$, e a densidade a posteriori marginal para $\beta$ (3.4) deve ser escrita na forma,

$$
\pi(\beta / \text { dados }) \propto \frac{e^{-\beta \tilde{p} a_{1}}}{\left\{\sum_{i=1}^{k} V_{i}^{\tilde{p}} A_{i}(\tilde{p}) e^{\frac{-\beta \tilde{p}}{V_{i}}}\right\}^{r \tilde{p}}}
$$

onde $-\infty<\beta<\infty$ e $A_{i}(\tilde{p})=\sum_{j=1}^{k} t_{i j}^{* \bar{p}}+\left(n_{i}-r_{i}\right) t_{i r_{i}}^{* \tilde{p}}$. O mesmo procedimento deve ser adotado às outras expressões do Capítulo 3.

\subsubsection{Densidade a Posteriori Conjunta para $\theta_{1}^{*}$ e $p$}

Em geral estamos interessados em inferências sobre o tempo médio de sobrevivência dos componentes, $\theta_{1}^{*}$, sob o nivel de estresse usual, $V_{i}$, considerando o parâmetro de forma da distribuição de Weibull, $p$, desconhecido.

O tempo médio de sobrevivência de um componente com distribuição de Weibull com parâmetro $\lambda_{i}^{*}$ e $p$ é dado por, $\theta_{i}^{*}=\frac{\Gamma(1+1 / p)}{\lambda_{i}^{*}}$. Como $\lambda_{i}^{*}=V_{i} \exp \{\alpha-$ $\left.\beta / V_{i}\right\}$, temos,

$$
\theta_{i}^{*}=\frac{e^{\beta / V_{i}} \Gamma(1+1 / p)}{V_{1} e^{\alpha}}
$$

Considerando a transförmação de variáveis $\theta_{1}^{*}=\frac{e^{\beta / v_{1}} \Gamma(1+1 / p)}{V_{1} e^{\alpha}}, \beta=\beta \mathrm{e}$ $p=p$, a densidade a priori para $\theta_{1}, \beta$ e $p$ é dada $(\operatorname{de}(5.12))$ por,

$$
\pi\left(\theta_{1}^{*}, \beta, p\right) \propto \frac{p}{\theta_{1}^{*}},
$$


onde $\theta_{1}^{*}, p>0$ e $-\infty<\beta<\infty$, e a densidade a posteriori conjunta para $\theta_{1}^{*}, \beta$ e $p \dot{\mathbf{e}}$ dada (de(5.13)) por,

$$
\left.\begin{array}{c}
\pi\left(\theta_{1}^{*}, \beta, p / \text { dados }\right) \propto p^{r+1} \theta_{1}^{*-(p r+1)}\left(\prod_{i=1}^{k} \prod_{j=1}^{r_{i}} t_{i j}^{* p-1}\right) \\
\left(\prod_{i=1}^{k} V_{i}^{p r_{i}}\right) V_{1}^{-p r}[\Gamma(1+1 / p)]^{p r} \\
\exp \left\{-p \beta\left(a_{1}-\frac{r}{V_{1}}\right)-\theta_{1}^{*-p}[\Gamma(1+1 / p)]^{p} \sum_{i=1}^{k}\left(\frac{V_{i}}{V_{1}}\right)^{p} A_{i}(p) e^{\beta p\left(\frac{1}{V_{1}}-\frac{1}{v_{i}}\right)}\right\},
\end{array}\right\}
$$

onde $\theta_{1}^{*}, p>0$ e $-\infty<\beta<\infty$.

A densidade a posteriori conjunta marginal para $\theta_{1}^{*}$ e $p$ aproximada pelo método de Laplace completamente exponencial (ver por exemplo, Tierney e Kadane, 1986; Apêndice 2) é dada por,

$$
\begin{aligned}
& \pi\left(\theta_{1}^{*}, p / d a d o s\right) \propto p^{r} \theta_{1}^{*-p(r-1 / 2)-1}\left(\prod_{i=1}^{k} \prod_{j=1}^{r_{i}} t_{i j}^{* p-1}\right) \\
& \left(\prod_{i=1}^{k} V_{i}^{p r_{i}}\right) V_{1}^{-p r}[\Gamma(1+1 / p)]^{p(r-1 / 2)} . \\
& \left.\cdot \frac{\exp \left\{-\hat{\beta} p\left(a_{1}-\frac{r}{V_{1}}\right) \theta_{i}^{*-p}[\Gamma(1+1 / p)]^{p} \sum_{i=1}^{k}\left(\frac{V_{i}}{V_{1}}\right)^{p} A_{i}(p) e^{\dot{\beta} p\left(\frac{1}{V_{1}}-\frac{1}{V_{i}}\right)}\right\}}{\left.\left\{\sum_{i=1}^{k} \frac{V_{i_{i}}}{V_{1}}\right)^{p} A_{i}(p)\left(\frac{1}{V_{1}}-\frac{1}{V_{i}}\right)^{2} e^{\dot{\beta} p\left(\frac{\partial_{1}}{V_{1}}-\frac{1}{V_{i}}\right)}\right\}^{1 / 2}},\right\}
\end{aligned}
$$

onde $\theta_{1}^{*}, p>0$ e $\hat{\beta}$ é o valor que maximiza

$$
-n h_{\theta_{i}, p}(\beta)=-\beta p\left(a_{1}-\frac{r}{V_{1}}\right)-\theta_{1}^{*-p}[\Gamma(1+1 / p)]^{p} \sum_{i=1}^{k}\left(\frac{V_{i}}{V_{1}}\right)^{p} A_{i}(p) e^{\beta p\left(\frac{1}{v_{1}}-\frac{1}{V_{i}}\right)}
$$

$\operatorname{Com} p=1$, a densidade a posteriori (5.19) reduz-se à densidade a posteriori marginal para $\theta_{1}(3.9)$. 


\section{Capítulo 6}

\section{Testes de Sobrevivência Acelerados com uma Relação Estresse-Resposta Geral}

Nos capítulos anteriores, considerando uma distribuição Exponencial para os tempos de sobrevivência, temos o desenvolvimento da metodologia clássica de análise do modelo de Eyring com uma variável estresse e considerações sobre o uso de parametrizações que possibilitam a obtenção de uma parametrização assintoticamente independente (ver Capítulo 2). Também, obtemos (ver Capitulo 3) uma Análise Bayesiana deste modelo, considerando uma densidade a priori não informativa de Jeffreys e explorando o uso do método de Laplace para aproximar integrais. Observamos que a parametrização que implica em uma densidade a priori não-informativa de Jeffreys localmente uniforme, "leva" a resultados satisfatórios do ponto de vista da aproximação de Laplace. No Capítulo 4, determinamos um procedimento de controle de qualidade através do uso da densidade preditiva de uma observação futura.

Considerando uma distribuição de Weibull para os tempos de sobrevivência, no Capítulo 5, obtemos uma Análise Bayesiana do modelo de Eyring, utilizando a 
regra multiparamétrica de Jeffreys para achar a densidade a priori ñ̃n-informativa dos parâmetros.

Até o momento, a mesma relação estresse-resposta foi considerada (modelo de Eyring com uma variável estresse), limitando, de certa forma, as análises de testes de sobrevivência acelerados que consideram uma única variável de estresse. De modo a suprimir tal limitação, consideramos, neste capitulo, uma relação estresse-resposta geral que inclue os modelos padrões (Potência, Arrenhius e Eyring com uma variável estresse) como casos particulares, com a qual são apresentadas metodologias de análise válidas para os três modelos em questão.

\subsection{A Relação Estresse-Resposta Geral}

As relações estresse-resposta mais comumente encontrados na literatura e utilizados na prática em testes acelerados que consideram somente uma variável física de estresse (modelo de Potência (1.3), Arrenhius (1.4) e Eyring (1.5) podem ser expressas como casos particulares de relação estresse-resposta geral, dada por (ver por exemplo, Klein e Basu, 1981; Barbosa e Louzada Neto, 1991),

$$
\begin{gathered}
\lambda^{*}=\exp \left\{-\left(Z+\beta_{0}+\beta_{1} X\right)\right\} \\
\text { onde } X=\left\{\begin{array}{cc}
-\log V, & \text { para o modelo Potência } \\
\frac{1}{V}, & \text { para os modelos Arrenhius e Eyring }
\end{array}\right. \\
Z=\left\{\begin{array}{cc}
-\log V, & \text { para o modelo de Eyring } \\
0, & \text { para os modelos Potência e Arrenhius }
\end{array}\right.
\end{gathered}
$$




$$
\beta_{0}=\left\{\begin{array}{cc}
\log \alpha, & \text { para o modelo Potência } \\
-\alpha, & \text { para os modelos Arrenhius e Eyring }
\end{array}\right.
$$

e $\beta_{1}=\beta$ para os três modelos (Potência, Arrenhius e Eyring).

Considerando a variável aleatória $T^{*}$ com distribuição de Weibull (definida no Capítulo anterior), com f.d.p. dada por,

$$
f\left(t^{*} ; \lambda^{*}, p\right)=\lambda^{*} p\left(\lambda^{*} t^{*}\right)^{p-1} \exp \left\{-\left(\lambda^{*} t^{*}\right)^{p}\right\}
$$

onde $t^{*}>0$ e $\lambda^{*}, p>0$, a relação estresse-resposta (6.1) com $k$ niveis aleatorizados de estresse $\left(X_{1}, X_{2}, \ldots, X_{k}\right)$, e um mecanismo de censura de Tipo II para os dados, a função de verossimilhança para os parâmetros $\beta_{0}, \beta_{1}$ e $p$ é dada por,

$$
\left.\begin{array}{c}
L\left(\beta_{0}, \beta_{1}, p\right)=p^{r}\left(\prod_{i=1}^{k} \prod_{j=1}^{r_{i}} t_{i j}^{* p-1}\right) \\
\exp \left\{-p a_{0}^{*}-p \beta_{0} r-p \beta_{1} a_{1}^{*}-e^{-p \beta_{0}} \sum_{i=1}^{k} A_{i}(p) e^{-p Z_{i}-p \beta_{1} X_{i}}\right\}
\end{array}\right\}
$$

onde $r=\sum_{i=1}^{k} r_{i}, a_{0}^{*}=\sum_{i=1}^{k} r_{i} Z_{i}, a_{1}^{*}=\sum_{i=1}^{k} r_{i} X_{i}$ e $A_{i}(p)=\sum_{j=1}^{k} t_{i j}^{*^{p}}+\left(n_{i}-r_{i}\right) t_{i r_{i}}^{*^{p}}$.

\subsection{Procedimento Bayesiano de Análise com uma Densidade de Weibull}

A densidade a priori para $\beta_{0}, \beta_{1}$ e $p$ pode ser escrita na forma, $\pi\left(\beta_{0}, \beta_{1}, p\right)=$ $\pi\left(\beta_{0}, \beta_{1} / p\right) \pi_{0}(p)$, e usando a regra multiparamétrica de Jeffreys, temos, $\pi\left(\beta_{0}, \beta_{1} / p\right) \propto$ $\left\{\operatorname{det} I_{p}\left(\beta_{0}, \beta_{1}\right)\right\}^{1 / 2}$, onde $I_{p}\left(\beta_{0}, \beta_{1}\right)$ é a matriz de informação de Fisher para $\beta_{0}$ e $\beta_{1}$ dado $p$. 
A matriz de informação de Fisher para $\beta_{0}$ e $\beta_{1}$ dado $p$ é dada por,

$$
I_{p}\left(\beta_{0}, \beta_{1}\right)=\left[\begin{array}{cc}
r p^{2} & a_{1}^{*} p^{2} \\
a_{1}^{*} p^{2} & a_{2}^{*} p^{2}
\end{array}\right]
$$

onde $r$ e $a_{1}^{*}$ são dados em (6.3) e $a_{2}^{*}=\sum_{i=1}^{k} r_{i} X_{i}^{2}$.

Assumindo $\pi_{0}(p) \propto \frac{1}{p}, p>0$, a densidade a priori para $\beta_{0}, \beta_{1}$ e $p$, baseada na regra multiparamétrica de Jeffreys é dada por,

$$
\pi\left(\beta_{0}, \beta_{1}, p\right) \propto p
$$

onde $p>0 \mathrm{e}-\infty<\beta_{0}, \beta_{1}<\infty$.

\subsubsection{Densidade a Posteriori Marginal para $p$<smiles>C1CCCC1</smiles>

Considerando-se a densidade a priori (6.5), a densidade a posteriori conjunta para $\beta_{0}, \beta_{1}, p$ é dada por,,

$$
\begin{gathered}
\pi\left(\beta_{0}, \beta_{1}, p / \text { dados }\right) \propto p^{r+1}\left(\prod_{i=1}^{k} \prod_{j=1}^{r_{i}} t_{i j}^{* p-1}\right) \exp \left\{-p a_{0}^{*}-p \beta_{0} r-p \beta_{1} a_{1}^{*}\right\} \\
\exp \left\{-e^{-p \beta_{0}} \sum_{i=1}^{k} A_{i}(p) e^{-p Z_{i}-p \beta_{1} X_{i}}\right\}
\end{gathered}
$$

onde $p>0 \mathrm{e}-\infty<\beta_{0}, \beta_{1}<\infty$.

A densidade a posteriori marginal para $p$, aproximada pelo método de Laplace completamente exponencial (ver Apêndice 2), é dada por, 


$$
\begin{aligned}
& \pi(p / \text { dados }) \propto \\
& \left\{\frac{p^{r-1} e^{-p\left(a_{0}^{*}+\dot{\beta}_{1} a_{i}^{*}\right)}\left(\prod_{i=1}^{k} \prod_{j=1}^{r_{i}} t_{i j}^{* p-1}\right)}{\left\{r^{2} \frac{\sum_{i=1}^{k} A_{i}(p) x_{i}^{2} e^{-p\left(z_{i}+\dot{\beta}_{1} x_{i}\right)}}{\sum_{i=1}^{k} A_{i}(p) e^{-p\left(Z_{i}+\dot{\beta}_{1} x_{i}\right)}-a_{i}^{2}}\right\}^{1 / 2}\left\{\sum_{i=1}^{k} A_{i}(p) e^{-p\left(z_{i}+\dot{\beta}_{1} x_{i}\right)}\right\}^{r}},\right.
\end{aligned}
$$

onde $p>0$ e $\hat{\beta}_{1}$ maximiza $g\left(\beta_{1}\right)=-r \log \left\{\frac{\sum_{i=1}^{k} A_{i}(p) e^{-p\left(z_{i}+\beta_{1} x_{i}\right)}}{r}\right\}-p \beta_{1} a_{1}^{*}-r$, para cada valor fixo de $p$.

Observe que $\operatorname{com} X_{i}=\frac{1}{V_{i}}, Z_{i}=-\log V_{i}, \beta_{0}=-\alpha$ e $\beta_{1}=\beta$, temos (6.7) igual à densidade a posteriori marginal para $p$ (5.15).

\subsubsection{Densidade a Posteriori Conjunta para $\theta_{1}^{*}$ e $p$}

O tempo médio de sobrevivência sob o nível de estresse $X_{1}$ é dado por $\theta_{1}^{*}=$ $F^{*}(1+1 / p) / \lambda_{1}^{*}$, onde $(\operatorname{de}(6.1)) \lambda_{1}^{*}=\exp \left\{-\left(Z_{1}+\beta_{0}+\beta_{1} X_{1}\right)\right\}$

Considerando a trańsformação de variáveis $\theta_{1}^{*}=\Gamma(1+1 / p) \exp \left\{Z_{1}+\beta_{0}+\right.$ $\left.\beta_{1} X_{1}\right\}, \beta_{1}=\beta_{1}$ e $p=p$, a densidade a posteriori conjunta para $\theta_{1}^{*}, \beta$ e $p$ é dada (de(6.6)) por,

$$
\begin{aligned}
& \pi\left(\theta_{1}^{*}, \beta_{1}, p / \text { dados }\right) \propto p^{r+1} \theta_{1}^{*-(p r+1)}\left(\prod_{i=1}^{k} \prod_{j=1}^{r_{i}} t_{i j}^{* p-1}\right) e^{-p\left(a_{0}^{*}-r Z_{1}\right)}[\Gamma(1+1 / p)]^{p r} \\
& \cdot \exp \left\{-p \beta_{1}\left(a_{1}^{*}-r X_{1}\right)-\theta_{1}^{*-p}[\Gamma(1+1 / p)]^{p} \sum_{i=1}^{k} A_{i}(p) e^{-\left(Z_{i}-Z_{1}\right)+p \beta_{1}\left(X_{1}-X_{i}\right)}\right\}
\end{aligned}
$$

onde $\theta_{1}^{*}, p>0$ e $-\infty<\beta_{1}<\infty$.

A densidade a posteriori conjunta marginal para $\theta_{1}^{*}$ e $p$ aproximada pelo método de Laplace completamente exponencial é dada por, 


$$
\begin{aligned}
& \pi\left(\theta_{1}^{*}, p / \text { dados }\right) \propto p^{r} \theta_{1}^{*-p(r-1 / 2)-1}\left(\prod_{i=1}^{k} \prod_{j=1}^{r_{i}} t_{i j}^{* p-1}\right) e^{-p\left(a_{0}^{*}-r Z_{1}\right)}[\Gamma(1+1 / p)]^{p(r-1 / 2)}
\end{aligned}
$$

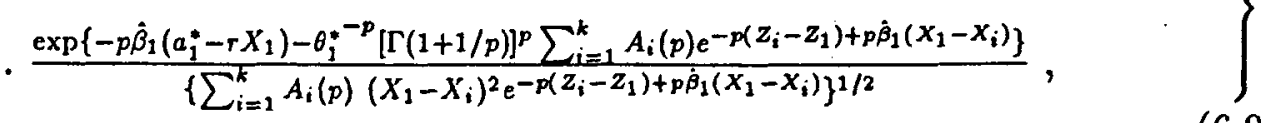

onde $\theta_{1}, p>0$ e $\hat{\beta}_{1}$ maximiza $-n h_{\theta_{i}^{*}, p}\left(\beta_{1}\right)=p \beta_{1}\left(a_{1}-r X_{1}\right)-\theta_{1}^{*^{-p}}[\Gamma(1+1 / p)]^{p}$ $\sum_{i=1}^{k} A_{i}(p) e^{-p\left(Z_{i}-Z_{1}\right)+p \beta_{1}\left(X_{1}-X_{i}\right)}$, para cada $\operatorname{par}\left(\theta_{1}^{*}, p\right)$ fixo.

$\operatorname{Com} X_{i}=1 / V_{i}, Z_{i}=-\log V_{i}, \beta_{0}=-\alpha$ e $\beta_{1}=\beta,(6.9)$ reduz-se à densidade a posteriori conjunta marginal (5.19).

\subsection{Análise Bayesiana assumindo uma Distribuição Exponencial para $T^{*}$}

Assumindo uma distribuição Exponencial para $T^{*}$ com parâmetro $\lambda^{*}(p=1$, em (6.2)), e considerando a densidade a priori não-informativa de Jeffreys localmente uniforme para $\beta_{0}$ e $\beta_{1}$, dada por, $\pi\left(\beta_{0}, \beta_{1}\right) \propto$ constante, $-\infty<\beta_{0}, \beta_{1}<\infty$, a densidade a posteriori conjunta para $\beta_{0}$ e $\beta_{1}$ é dada por,

$$
\pi\left(\beta_{0}, \beta_{1} / \text { dados }\right) \propto \exp \left\{-\beta_{0} r-\beta_{1} a_{1}^{*}-e^{-\beta_{0}} \sum_{i=1}^{k} A_{i} e^{-Z_{i}-\beta_{1} X_{i}}\right\}
$$

onde $-\infty<\beta_{0}, \beta_{1}<\infty$.

A densidade a posteriori marginal para $\beta_{1}$ é dada por,

$$
\pi\left(\beta_{1} / \text { dados }\right) \propto \frac{e^{-\beta_{1} a_{i}^{*}}}{\left\{\sum_{i=1}^{k} A_{i} e^{-Z_{i}-\beta_{1} X_{i}}\right\}^{r}}
$$


onde $-\infty<\beta_{1}<\infty$, e a densidade a posteriori marginal para $\beta_{0}$, aproximada pelo método de Laplace completamente exponencial (ver Apêndice 2), é dada por,

$$
\pi\left(\beta_{0} / d a d o s\right) \propto \frac{\exp \left\{-\beta_{0}(r-1 / 2)-\hat{\beta}_{1} a_{1}^{*}-e^{-\beta_{0}} \sum_{i=1}^{k} A_{i} e^{-Z_{i}-\hat{\beta}_{1} X_{i}}\right\}}{\left\{\sum_{i=1}^{k} A_{i} X_{i}^{2} e^{-Z_{i}-\hat{\beta}_{1} X_{i}}\right\}^{1 / 2}}
$$

onde $-\infty<\beta_{0}<\infty$ è $\hat{\beta}_{1}$ maximiza $-\beta_{1} a_{1}^{*}-e^{-\beta_{0}} \sum_{i=1}^{k} A_{i} e^{-Z_{i}-\beta_{1} X_{i}}$, para cada valor de $\beta_{0}$ fixo.

Considerando a transformação de variáveis $\theta_{1}^{*}=\exp \left\{Z_{1}+\beta_{0}+\beta_{1} X_{1}\right\}$ e $\beta_{1}=\beta_{1}$ a densidade a posteriori conjunta para $\theta_{1}^{*}$ e $\beta_{1}$ é dada $(\operatorname{de}(6.10))$ por,

$$
\pi\left(\theta_{1}^{*}, \beta_{1} / \text { dados }\right) \propto \theta_{1}^{*-(r+1)} \exp \left\{-\beta_{1}\left(a_{1}^{*}-r X_{1}\right)-\theta_{1}^{*-1} \sum_{i=1}^{k} A_{i} e^{-\left(Z_{i}-Z_{1}\right)+\beta_{1}\left(X_{1}-X_{i}\right)}\right\}
$$

onde $\theta_{1}^{*}>0$ e $-\infty<\beta_{1}<\infty$.



A densidade a posteriori marginal para $\theta_{1}^{*}$ aproximada pelo método de Laplace completamente exponencial (ver Apêndice 2) é dada por,

$$
\pi\left(\theta_{1}^{*} / d a d o s\right) \propto \frac{\theta_{1}^{*-(r+1 / 2)} \exp \left\{-\hat{\beta}_{1}\left(a_{1}^{*}-r X_{1}\right)-\theta_{1}^{*-1} \cdot \sum_{i=1}^{k} A_{i} e^{-\left(Z_{i}-Z_{1}\right)+\hat{\beta}_{1}\left(X_{1}-X_{i}\right)}\right\}}{\left\{\sum_{i=1}^{k} A_{i}\left(X_{1}-X_{i}\right)^{2} e^{-\left(Z_{i}-Z_{1}\right)+\hat{\beta}_{1}\left(X_{1}-X_{i}\right)}\right\}^{1 / 2}}
$$

onde $\theta_{1}^{*}>0$ e $\hat{\beta}_{1}$ maximiza $-\beta_{1}\left(a_{1}^{*}-r X_{1}\right)-\theta_{1}^{*-1} \sum_{i=1}^{k} A_{i} e^{-\left(Z_{i}-Z_{1}\right)+\beta_{1}\left(X_{1}-X_{i}\right)}$, para cada valor fixo de $\theta_{1}^{*}$.

Observe que com $X_{i}=1 / V_{i}, Z_{i}=-\log V_{i}, \beta_{0}=-\alpha$ e $\beta_{1}=\beta_{1}$, as expressões obtidas nesta seção tornam-se iguais as obtidas no Capítulo 3 (ver seções $3.2,3.3$ e 3.4$)$. 
Dessa forma, temos liberdade na análise de testes de súz: zvivência acęlerados, no sentido de podermos considerar quaisquer dos três modelos com uma única variável de estresse (Potência, Arrenhius e Eyring). Também, a distribuiçäăo de sobrevivência tanto pode ser Weibull, como Exponencial.

Cabe ressaltar que, como no capítulo anterior, considerando $T=T^{*^{*}}$ com uma distribuição Exponencial com parâmetro $\lambda=\lambda^{*^{p}}$, também podemos considerár uma Análise Bayesiana do modelo geral (6.1), onde $p$ pode ser dado pela moda da densidade posterior marginal para $p(6.7)$.

\subsection{A Função de Confiabilidade em Experimen- tos Acelerados}

Em geral, os pesquisadores industriais; além do usual interesse em inferências sobre o tempo médio de sobrevivência, $\theta_{1}^{*}$, de um componente manufaturado, sob o nível usual de estresse, $X_{1}$, têm interesse nas inferências ş̧́bre a probabilidade de um particular componente sobreviver pelo menos um determinado tempo $t_{1}^{*}$, considerando o nivel $X_{1}$, dada por,

$$
R_{1}^{*}=P\left(T^{*} \geq t_{1}^{*}\right)
$$

A probabilidade (6.15) é dita a função de confiabilidade (ver por exemplo, Lawless, 1982) da variável aleatória $T^{*}$ no nivel usual de estresse $X_{1}$.

Considerando $T^{*}$ com uma distribuição de Weibull com parâmetros $\lambda^{*}$ e $p$ (ver (6.2)), a funç̧ão de confiabilidade (6.15) é dada por (ver por exemplo, Lawless, 1982), 


$$
R_{1}^{*}=e^{-\left(\lambda_{i}^{*} t_{1}^{*}\right)^{p}}
$$

(observe que nos Capítulos 2 e 5 , já havíamos definido, resumidamente, a função de confiabilidade (ver seções 2.2 e 5.1 )).

Nesta seção, (6.16) é determinada, considerando-se a relação estresse-resposta (6.1), tanto para uma distribuição de sobrevivência de Weibull, quanto para a Exponencial (quando $p=1$ ).

\subsubsection{Densidade a Posteriori Conjunta para a Função de Confiabilidade $R_{1}^{*}$ e para o Parâmetro de Forma $p$ da Distribuição de Weibull}

Como $\theta_{1}^{*}=\frac{\Gamma(1+1 / p)}{\lambda_{i}^{*}}$, considerando a transformação de variáveis $R_{1}^{*}=$ $\therefore \exp \left\{-\theta_{1}^{*-p}[\Gamma(1+1 / p)]^{p} t^{* p}\right\}, \beta_{1}=\beta_{1}$ e $p=p$, a densidade a posteriori conjunta 7 para $R_{1}^{*}, \beta_{1}, p$ é dada (de (6.8)) por,

$$
\left.\begin{array}{l}
\pi\left(R_{i}^{*}, \beta_{1}, p / \text { dados }\right) \propto \\
\frac{p^{r}\left(\prod_{i=1}^{k} \prod_{j=1}^{r i} t_{i j}^{p-1}\right) \exp \left\{-p \beta_{1}\left(a_{i}^{*}-r X_{1}\right)-t_{i}^{*-p}\left(-\log R_{i}^{*}\right) \sum_{i=1}^{k} A_{i}(p) e^{\left.-p\left(Z_{i}-z_{1}\right)+p \beta_{1}\left(x_{1}-z_{i}\right)\right\}}\right.}{R_{i}^{*}\left(-\log R_{i}^{*}\right)^{-(r-1)} t_{i}^{*-p r} \exp \left\{-p\left(a_{0}^{*}-r Z_{1}\right)\right\}},
\end{array}\right\}
$$

onde $0 \leq R_{1}^{*} \leq 1, p>0 \mathrm{e}-\infty<\beta_{1}<\infty$.

Usando o método de Laplace completamente exponencial (ver Apêndice 2), a densidade a posteriori conjunta marginal aproximada para $R_{1}^{*}$ e $p$ é dada por, 


$$
\left.\begin{array}{l}
\pi\left(R_{1}^{*}, p / \text { dados }\right) \propto \\
\frac{p^{r-1}\left(\prod_{i=1}^{k} \prod_{j=1}^{r_{i}} t_{i j}^{p-1}\right) \exp \left\{-p \hat{\beta}_{1}\left(a_{i}^{*}-r X_{1}\right)-t_{1}^{*-p}\left(-\log R_{1}^{*}\right) \sum_{i=1}^{k} A_{i}(p) e^{\left.-p\left(Z_{i}-z_{1}\right)+p \dot{\beta}_{1}\left(X_{1}-x_{i}\right)\right\}}\right.}{R_{1}^{*}\left(-\log R_{1}^{*}\right)^{-(r-3 / 2)} t_{i}^{(p r-1 / 2)} e^{-p\left(a_{j}^{*}-r Z_{1}\right)}\left\{\sum_{i=1}^{k} A_{i}(p)\left(X_{1}-X_{i}\right)^{2} e^{\left.-p\left(Z_{i}-Z_{1}\right)+p \dot{p}_{1}\left(X_{1}-X_{i}\right)\right\}^{1 / 2}}\right.},
\end{array}\right\}
$$

onde $0 \leq R_{1}^{*} \leq 1, p>0$ e $\hat{\beta}$ maximiza

$$
-n h_{R_{1}^{*}, p}\left(\beta_{1}\right)=-p \beta_{1}\left(a_{1}^{*}-r X_{1}\right)-\left(-\log \left(R_{1}^{*}\right)\right) t_{1}^{*-p} \sum_{i=1}^{k} A_{i}(p) e^{-p\left(Z_{i}-Z_{1}\right)+p \beta_{1}\left(X_{1}-X_{i}\right)},
$$

para cada valor fixo do $\operatorname{par}\left(R_{1}^{*}, p\right)$.

\subsubsection{Densidade a Posteriori para $R_{1}^{*}$ Assumindo uma Dis- tribuição Exponencial para $T^{*}$}

Assumindo $T_{:}^{*}$ com uma distribuição Expọnenciạl com parâmețro $\lambda^{*}(p=1$ em (6.2)), a densidade a posteriori conjunta para $R_{1}^{*}$ e $\beta_{1}$ é dada (de (6.13)) por,

$\pi\left(R_{1}^{*}, \beta_{1} /\right.$ dados $) \propto \frac{\left.\exp \left\{-\beta_{1}\left(a_{1}^{*}-r X_{1}\right)-\left(-\log R_{1}^{*}\right)\right) t_{1}^{*-1} \sum_{i=1}^{k} A_{i} e^{-\left(Z_{i}-Z_{1}\right)+\beta_{1}\left(X_{1}-X_{i}\right)}\right\}}{R_{1}^{*}\left(-\log \left(R_{1}^{*}\right)\right)^{-(r-1)}}$

onde $0 \leq R_{\mathrm{i}}^{*} \leq 1 \mathrm{e}-\infty<\beta_{1}<\infty$

Definindo $-n h_{R_{1}^{*}}\left(\beta_{1}\right)=-\beta_{1}\left(a_{1}^{*}-r X_{1}\right)-\left(-\log \left(R_{1}^{*}\right)\right) t_{1}^{*-1} \sum_{i=1}^{k} A_{i} e^{-\left(Z_{i}-Z_{1}\right)+\beta_{1}\left(X_{1}-X_{i}\right)}$,

a densidade a posteriori marginal para $R_{1}^{*}$, aproximada pelo método de Laplace completamente exponencial (ver Apêndice 2), é dada por,

$$
\left.\begin{array}{l}
\pi\left(R_{1}^{*} / \text { dados }\right) \propto \\
\frac{\left(-\log R_{i}^{*}\right)^{r-\frac{3}{2}} \exp \left\{-\hat{\beta}_{1}\left(a_{i}^{*}-r X_{1}\right)-\left(-\log \left(R_{i}^{*}\right)\right) t_{i}^{*} \sum_{i=1}^{k} A_{i} e^{\left.-\left(Z_{i}-Z_{1}\right)+\hat{\beta}_{1}\left(X_{1}-x_{i}\right)\right\}}\right.}{R_{i}^{*}\left(\sum_{i=1}^{k} A_{i}\left(X_{1}-X_{i}\right)^{2} e^{\left.-\left(Z_{i}-Z_{1}\right)+\hat{\beta}_{1}\left(X_{1}-X_{i}\right)\right\}^{\frac{1}{2}}}\right.}
\end{array}\right\}
$$


onde $0 \leq R_{1}^{*} \leq 1$ e $\hat{\beta}_{1}$ maximiza $-n h_{R_{i}^{*}}\left(\beta_{1}\right)$. 


\section{Capítulo 7}

\section{Experimentos Acelerados com Niveis de Estresse Somente Maiores que os Usuais}

Em muitas situações práticas, não é possível a condução de um experimento acelerado, considerando-se o nivel usual da variável física de estrèsse mesmo se considerarmos um número grande de censuras neste nível. Neste contexto, observe que nos capítulos anteriores, apesar de sempre $n_{1}$ componentes serem testados sob o nivel usual de estresse, se temos dados somente com $k$ níveis de estresse estritamente maiores que os usuais, $i=2,3, \ldots, k$, como assumimos que as relações estresseresposta envolvidas (ver (1.3), (1.4) e (1.5), ou (6.1)) são válidas para esses níveis e também para o nivel usual, podemos inferir sob esse nível, $\mathbf{e}$ as expressões obtidas serão iguais as obtidas anteriormente, $\operatorname{com} i=2,3, \ldots, k$.

Particularmente, assumindo dois níveis de estresse maiores que o usual e um mecanismo de censura de Tipo II para os dados, desenvolvemos, neste capítulo, uma Anålise Bayesiana da relação estresse-resposta geral, proposta no capítulo anterior, mostrando que é possível a obtenção de inferências sobre o tempo médio de sobrevivência, $\theta_{1}$, sob o nível usual de estresse, mesmo sem termos dados sob esse 
nî́vel.

\subsection{Determinação do Modelo Considerando so- mente níveis de estresse maiores que os usu- ais}

Considere um teste de sobrevivência acelerado com dois niveis de estresse, $X_{2}$ e $X_{3}$, maiores que o usual, $X_{1}(>0)$, sob os quais $n_{i}(i=2,3)$ unidades são testadas, e que o experimento termina quando $r_{i}$ falhas ocorrem. Também, considere a variável aleatória $T^{*}$ com uma densidade de Weibull com parâmetros $\lambda^{*}$ e $p$ $(\operatorname{ver}(6.2))$.

Assumindo que o parâmetro de forma, $p$, é diferente para cada um dos dois níveis de estresse e que a variável estresse $X$ relaciona-se com $\lambda^{*}$ através de (6.1), a função de verossimilhança para $\lambda_{i}^{*}$ e $p_{i}$, no i-ésimo nível de estresse, é dado por,

$$
L_{i}\left(\lambda_{i}^{*}, p_{i}\right)=p_{i}^{r_{i}} \lambda_{i}^{p_{i}^{p_{i}}}\left(\prod_{j=1}^{r_{i}} t_{i j}^{*_{i}^{p_{i}-1}}\right) e^{-\lambda_{i}^{p_{i}} A_{i}\left(p_{i}\right)}
$$

onde $A_{i}\left(p_{i}\right)=\sum_{j=1}^{r_{i}} t_{i j}^{* p_{i}}+\left(n_{i}-r_{i}\right) t_{i r_{i}}^{p_{i}}$.

Com dados sob os dois níveis de estresse, $X_{2}$ e $X_{3}$, a função de verossimilhança para $\beta_{0}, \beta_{1}, p_{2}, p_{3}$ é dada (de(6.1) è (7.1)) por,

$$
\left.\begin{array}{c}
L\left(\beta_{0}, \beta_{1}, p_{2}, p_{3}\right)=\left(\prod_{i=2}^{3} p_{i}^{r_{i}}\right)\left(\prod_{i=2}^{3} \prod_{j=1}^{r_{i}} t_{i j}^{*_{i}-1}\right) \\
\exp \left\{\sum_{i=2}^{3} p_{i} r_{i} Z_{i}-\beta_{0} \sum_{i=2}^{3} p_{i} r_{i}-\beta_{1} \sum_{i=2}^{3} p_{i} r_{i} X_{i}\right\} \\
\exp \left\{-\sum_{i=2}^{3} A_{i}\left(p_{i}\right) e^{-p_{i} Z_{i}-\beta_{0} p_{i}-\beta_{1} p_{i} X_{i}}\right\}
\end{array}\right\}
$$




\subsection{Uma Análise Bayesiana do Modelo}

A densidade a priori para $\beta_{0}, \beta_{1}, p_{2}$ e $p_{3}$ pode ser escrita na forma, $\pi\left(\beta_{0}, \beta_{2}, p_{2}, p_{3}\right)=$ $\pi\left(\beta_{0}, \beta_{1} / p_{2}, p_{3}\right) \pi_{0}\left(p_{2}, p_{3}\right)$. Usando a regra multiparamétrica de Jeffreys, temos, $\pi\left(\beta_{0}, \beta_{1} / p_{2}, p_{3}\right) \propto\left\{\operatorname{det} I_{p_{2}, p_{3}}\left(\beta_{0}, \beta_{1}\right)\right\}^{1 / 2}$, onde $I_{p_{2}, p_{3}}\left(\beta_{0}, \beta_{1}\right)$ é a matriz de informação de Fisher para $\beta_{0}$ e $\beta_{1}$ dado $p_{2}$ e $p_{3}$, dada por,

$$
I_{p_{2}, p_{3}}\left(\beta_{0}, \beta_{1}\right)=\left[\begin{array}{cc}
\sum_{i=2}^{3} p_{i}^{2} r_{i} & \sum_{i=2}^{3} p_{i}^{2} r_{i} X_{i} \\
\sum_{i=2}^{3} p_{i}^{2} r_{i} X_{i} & \sum_{i=2}^{3} p_{i}^{2} r_{i} X_{i}^{2}
\end{array}\right],
$$

isto é,

$$
\pi\left(\beta_{0}, \beta_{1} / p_{2}, p_{3}\right) \propto p_{2} p_{3}
$$

onde $-\infty<\beta_{0}, \beta_{1}<\infty$

Assumindo densidades a priori localmente uniformes e independentes para $\log p_{2}$ e log $p_{3}, \pi_{0}\left(p_{2}, p_{3}\right) \propto\left(p_{2}, p_{3}\right)^{-1}, p_{2}, p_{3}>0$, temos,

$$
\pi\left(\beta_{0}, \beta_{1}, p_{2}, p_{3}\right) \propto \text { constante, }
$$

onde $p_{2}, p_{3}>0 \mathrm{e}-\infty<\beta_{0}, \beta_{1}<\infty$.

A densidade a posteriori conjunta para $\beta_{0}, \beta_{1}, p_{2}$ e $p_{3}$ é dada (de(7.2) e (7.3)) por,

$$
\begin{gathered}
\pi\left(\beta_{0}, \beta_{1}, p_{2}, p_{3} / \text { dados }\right) \propto \\
\left(\prod_{i=2}^{3} p_{i}^{r_{i}}\right)\left(\prod_{i=2}^{3} \prod_{j=1}^{r_{i}} t_{i j}^{p_{i}-1}\right) \exp \left\{-\sum_{i=2}^{3} p_{i} r_{i} Z_{i}-\beta_{0} \sum_{i=2}^{3} p_{i} r_{i}-\beta_{1} \sum_{i=2}^{3} p_{i} r_{i} X_{i}\right\} \\
\exp \left\{-\sum_{i=2}^{3} A_{i}\left(p_{i}\right) e^{-p_{i} Z_{i}-\beta_{0} p_{i}-\beta_{1} p_{i} X_{i}}\right\}
\end{gathered}
$$


onde $p_{2}, p_{3}>0$ e $-\infty<\beta_{0}, \beta_{1}<\infty$.

Usando o método de Laplace completamente exponencial para aproximação de integrais, a densidade a posteriori conjunta marginal aproximada para $p_{2}$ e $p_{3}$ é dada $(\operatorname{de}(7.4))$ por

$$
\begin{aligned}
& \pi\left(p_{2}, p_{3} / \text { dados }\right) \propto \\
& \frac{\left(\prod_{i=2}^{3} p_{i}^{r_{i}}\right)\left(\prod_{i=2}^{3} \prod_{i=1}^{r_{i}} t_{i j}^{p_{i}-1}\right) \exp \left\{-\sum_{i=2}^{3} p_{i} r_{i} Z_{i}-\hat{\beta}_{0} \sum_{i=2}^{3} p_{i} r_{i}-\hat{\beta}_{1} \sum_{i=2}^{3} A_{i}\left(p_{i}\right) e^{\left.-p_{i} r_{i}-\dot{\beta}_{0} p_{i}-\dot{\beta}_{1} p_{i} x_{i}\right\}}\right.}{\left\{\sum_{i=2}^{3} p_{i}^{2} A_{i}\left(p_{i}\right) e^{-p_{i} z_{i}-\hat{\beta}_{0} p_{i}-\beta_{1} p_{i} X_{i}} \sum_{i=2}^{3} p_{i}^{2} X_{i}^{2} A_{i}\left(p_{i}\right) e^{p_{i}} Z_{i}-\hat{\beta}_{0} p_{i}-\dot{\beta}_{1} p_{i} X_{i}-\left(\sum_{i=2}^{3} p_{i}^{2} X_{i} A_{i}\left(p_{i}\right) e^{\left.\left.-p_{i} Z_{i}-\hat{\beta}_{0} p_{i}-\beta_{1} p_{i} x_{i}\right)^{2}\right\}^{1 / 2}}\right.\right.}
\end{aligned}
$$

onde $p_{2}, p_{3}>0$ e o $\operatorname{par}\left(\hat{\beta}_{0}, \hat{\beta}_{1}\right)$ maximiza $-n h_{p_{2}, p_{3}}\left(\beta_{0}, \beta_{1}\right)=-\beta_{0} \sum_{i=2}^{3} p_{i} r_{i}-\beta_{1} \sum_{i=2}^{3} p_{i} r_{i} X_{i}-$ $\sum_{i=2}^{3} A_{i}\left(p_{i}\right) e^{-p_{i} Z_{i}-\beta_{0} p_{i}-\beta_{1} p_{i} X_{i}}$, para cada $\operatorname{par}\left(p_{2}, p_{3}\right)$ fixo.

Observe que assumindo $p_{2}=p_{3}=p$ e uma densidade a priori localmente uniforme para $\log p, \pi_{0}(p) \propto 1 / p, p>0$, a densidade a priori não informativa para $\beta_{0}, \beta_{1}$ e $p$, baseada na regra multiparamétrica de Jeffreys $\left(\pi\left(\beta_{0}, \beta_{1} / p\right) \propto\right.$ $\left.\left\{\operatorname{det} I_{p}\left(\beta_{0}, \beta_{1}\right)\right\}^{1 / 2}\right)$, é dada por, $\pi\left(\beta_{0}, \beta_{1}, p\right) \propto p, p>0$, e a densidade a posteriori marginal para $p$ é dada por,

$$
\pi(p / d a d o s) \propto \frac{p^{r^{\prime}-1} e^{-p\left(a_{0}^{a^{\prime}}+\hat{\beta}_{1} a_{i}^{\prime}\right)}\left(\prod_{i=2}^{3} \prod_{j=1}^{r_{i}} t_{i j}^{* p-1}\right)}{\left\{r^{\prime 2} \sum_{i=2}^{3} A_{i}(p) X_{i}^{2} e^{-p\left(Z_{i}+\dot{\beta}_{1} X_{i}\right)}-a_{1}^{*^{\prime 2}}\right\}^{1 / 2}\left\{\sum_{i=2}^{3} A_{i}(p) e^{-p\left(Z_{i}+\hat{\beta}_{1} X_{i}\right)}\right\}^{r^{\prime}}},
$$

onde $p>0, \hat{\beta}$ maximiza $-n h_{p}\left(\hat{\beta}_{0}, \beta_{1}\right)=g\left(\beta_{1}\right)=-r^{\prime} \log \left\{\frac{\sum_{i=2}^{3} A_{i}(p) e^{-p\left(Z_{i}+\beta_{1} x_{i}\right)}}{r^{\prime}}\right\}-$ $p \beta_{1} a_{1}^{*^{\prime}}-r^{\prime}$ para cada valor fixo de $p, r^{\prime}=\sum_{i=2}^{3} r_{i}, a_{0}^{*^{\prime}}=\sum_{i=2}^{3} r_{i} Z_{i}$ e $a_{1}^{*^{\prime}}=\sum_{i=2}^{3} r_{i} X_{i}$ (observe que esta expressão é equivalęnte a (6.7)).

Considerando o par $\left(p_{2}, p_{3}\right)$ igual a moda da densidade a posteriori conjunta (7.5), $\left(\tilde{p}_{2}^{\prime \prime}, \tilde{p}_{3}^{\prime \prime}\right)$, podemos fazer a transformação $T_{2}^{* \tilde{p}^{\prime \prime}}$ e $T_{3}^{\psi^{\hat{p}^{\prime \prime}}}, \operatorname{com} \tilde{p}^{\prime \prime}=\frac{\sum_{i=2}^{3} r_{i} \tilde{p}_{i}^{\prime \prime}}{\sum_{i=2}^{3} r_{i}}$ (média ponderada dos $\left.\tilde{p}_{i}^{\prime \prime}, i=2,3\right)$, onde essas variáveis aleatórias têm distribuição Expo- 
nencial comparâmetros $\lambda_{2}^{\boldsymbol{*}^{\prime \prime}}$ e $\lambda_{3}^{\boldsymbol{\tilde { p }}^{\prime \prime}}$, respectivamente, e considerar a análise Bayesiana padrão para o modelo geral, dada na seção seguinte, assumindo $p_{1}$ conhecido dado por $p_{1}=\tilde{p}^{\prime \prime}$.

Além disso, observe que a densidade a posteriori conjunta (7.5) pode ser utilizada para verificarmos se o parâmetro $p$ pode ser considerado igual nos níveis de estresse.

\subsection{Análise Bayesiana assumindo $p_{2}=p_{3}=1$}

Assumindo $p_{2}=p_{3}=1$, isto é; $T^{*}$, com uma distribuição. Exponencial com parâmetro $\lambda^{*}$, a função de verossimilhança para $\beta_{0}$ e $\beta_{1}$ é dada $(\operatorname{de}(7.2))$ por,

$$
L\left(\beta_{0}, \beta_{1}\right)=\exp \left\{-a_{0}^{*^{\prime}}-\beta_{0} r^{\prime}-\beta_{1} a_{1}^{*^{\prime}}-e^{-\beta_{0}} \sum_{i=2}^{3} A_{i} e^{-Z_{i}-\beta_{1} X_{i}}\right\}
$$

onde $r^{\prime}, a_{0}^{*^{\prime}}$ e $a_{1}^{*^{\prime}}$ são dados em $(7.6)$ e $A_{i}=\sum_{j=1}^{r_{i}} t_{i j}^{*}+\left(n_{i}-r_{i}\right) t_{i r_{i}}^{*}$.

Como o interesse é inferir sobre o tempo médio de sobrevivência sob o nível usual de estresse $X_{1}$, dado por $\theta_{1}^{*}=1 / \lambda_{1}^{*}$, mostremos o seguinte resultado que relaciona o parâmetro $\lambda_{1}^{*}$ com os parâmetros, $\lambda_{2}^{*} \mathrm{e} \lambda_{3}^{*}$.

Resultado: Se a relação entre $\lambda^{*}$ e $X$, dada $(\operatorname{de}(6.1))$ por, $\lambda^{*}=\exp \left\{-\left(Z+\beta_{0}+\right.\right.$ $\left.\left.\beta_{1} X\right)\right\}$, é válida para os três niveis $X_{1}<X_{2}<X_{3}$, tal que $X_{1}>0$, então,

$$
\lambda_{1}^{*}=\frac{\lambda_{2}^{* Y_{1}}}{\lambda_{3}^{* Y_{2}^{\prime}}} \exp \left\{-Z_{1}+Y_{1} Z_{2}-Y_{2} Z_{3}\right\}
$$

wiide $Y_{1}=\frac{X_{3}-X_{1}}{X_{3}-X_{2}}$ e $Y_{2}=\frac{X_{2}-X_{1}}{X_{3}-X_{2}}$ 
Prova:

Assumindo (6.1) válida para os três niveis de estresse, temos,

$$
\begin{aligned}
& \lambda_{1}^{*}=\exp \left\{-\left(Z_{1}+\beta_{0}+\beta_{1} X_{1}\right)\right\}, \\
& \lambda_{2}^{*}=\exp \left\{-\left(Z_{2}+\beta_{0}+\beta_{1} X_{2}\right)\right\}, \\
& \lambda_{3}^{*}=\exp \left\{-\left(Z_{3}+\beta_{0}+\beta_{1} X_{3}\right)\right\} .
\end{aligned}
$$

Resolvendo as equações (7.10) e (7.11) com respeito a $\beta_{0}$ e $\beta_{1}$, obtemos,

$\beta_{0}=\log \left(\frac{\lambda_{3}^{\frac{x_{3}}{x_{3}-x_{2}}}}{\lambda_{2}^{x_{3}-X_{3}}}\right)-\left(\frac{X_{3}}{X_{3}-X_{2}}\right) Z_{2} \backslash+\left(\frac{X_{2}}{X_{3}-X_{2}}\right) Z_{3}$

$$
\beta_{1}=\log \left(\frac{\lambda_{2}^{*}}{\lambda_{3}^{*}}\right)^{\frac{1}{x_{3}-x_{2}}}-\frac{Z_{3}-Z_{2}}{X_{3}-X_{2}}
$$

Substituindo (7.12) e (7.13) em (7.9) obtemos (7.8). ㅁ

Igualando as expressões (7.8) e (7.9) (ver Resultado), temos,

$$
e^{-\beta_{0}}=\frac{\lambda_{2}^{*_{1}}}{\lambda_{3}^{*^{Y_{2}}}} \frac{\exp \left\{-Z_{1}+Y_{1} Z_{2}-Y_{2} Z_{3}\right\}}{\exp \left\{-\left(Z_{1}+\beta_{1} X_{1}\right)\right\}}
$$

Substituindo (7.14) em (7.7), temos, 


$$
\begin{aligned}
L\left(\lambda_{2}^{*}, \lambda_{3}^{*}, \beta_{1}\right) & \propto\left\{\frac{\lambda_{2}^{* Y_{1}}}{\lambda_{3}^{Y_{2}}} \frac{\exp \left\{-Z_{1}+Y_{1} Z_{2}-Y_{2} Z_{3}\right\}}{\exp \left\{-\left(Z_{1}+\beta_{1} X_{1}\right)\right\}}\right\}^{r^{\prime}} e^{-\beta_{1} a_{1}^{*^{\prime}}} . \\
& \exp \left\{-\left(\frac{\lambda_{2}^{Y_{1}}}{\lambda_{3}^{* Y_{2}}} \frac{\exp \left\{-Z_{1}+Y_{1} Z_{2}-Y_{2} Z_{3}\right\}}{\exp \left\{-\left(Z_{1}+\beta_{1} X_{1}\right)\right\}}\right)^{-1} \sum_{i=2}^{3} A_{i} e^{-Z_{i}-\beta_{1} X_{i}}\right\}(7 .
\end{aligned}
$$

Daí, como $\theta_{1}^{*}=1 / \lambda_{1}^{*}, \lambda_{1}^{*}=\frac{\lambda_{2}^{Y_{1}}}{\lambda_{3}^{Y_{2}}} \exp \left\{-Z_{1}+Y_{1} Z_{2}-Y_{2} Z_{3}\right\}$ (ver Resultado), a função de verossimilhança para $\theta_{1}^{*}$ e $\beta_{1}$ é dada (de(7.15)) por,

$$
L\left(\theta_{1}^{*}, \beta_{1}\right)=\theta_{1}^{*-r^{\prime}} \exp \left\{-\beta_{1}\left(a_{1}^{*^{\prime}}-r^{\prime} X_{1}\right)-\theta_{1}^{*-1} \sum_{i=2}^{3} A_{i} e^{-\left(Z_{i}-Z_{1}\right)+\beta_{1}\left(X_{1}-X_{i}\right)}\right\}
$$

Observe que poderíamos obter a mesma expressão notando que $\theta_{1}^{*}=\exp \left\{Z_{1}+\right.$ $\beta_{0}+\beta_{1} X_{1}$ \} (sob o nível usual de estresse) pode ser substituido diretamente na função de verossimilhança (7.7), uma vez que assumimos que a relação estresse- resposta (6.1) é válida para o nível usual, $X_{1}$.

A densidade a priori não-informativa de Jeffreys para $\theta_{1}^{*}$ e $\beta_{1}$ é dada por,

$$
\begin{aligned}
\pi\left(\theta_{1}^{*}, \beta_{1}\right) & \propto\left\{\operatorname{det} I\left(\theta_{1}^{*}, \beta_{1}\right)\right\}^{1 / 2} \\
& \propto \frac{1}{\theta_{1}^{*}}
\end{aligned}
$$

onde $\theta_{1}^{*}>0$ e $-\infty<\beta_{1}<\infty$.

Considerando a densidade a priori (7.17), a densidade a posteriori conjunta para $\theta_{1}^{*}$ e $\beta_{1}$ é dada por

$r\left(\theta_{1}^{*}, \beta_{1} /\right.$ dados $) \propto \theta_{1}^{*-\left(r^{\prime}+1\right)} \exp \left\{-\beta_{1}\left(a_{1}^{*^{\prime}}-r^{\prime} X_{1}\right)-\theta_{1}^{*-1} \sum_{i=2}^{3} A_{i} e^{-\left(Z_{i}-Z_{1}\right)+\beta_{1}\left(X_{1}-X_{i}\right)}\right\}$ 
Oinus $\theta_{1}^{*}>0$ e $-\infty<\beta_{1}<\infty$.

Definindo $-n h_{\theta_{i}^{*}}\left(\beta_{1}\right)=-\beta_{1}\left(a_{1}^{*^{\prime}}-r^{\prime} X_{1}\right)-\theta_{1}^{*-1} \sum_{i=1}^{k} A_{i} e^{-\left(Z_{i}-Z_{1}\right)+\beta_{1}\left(X_{1}-X_{i}\right)}$

a densidade a posteriori marginal de $\theta_{1}^{*}$ aproximada pelo método de Laplace completamente exponencial (ver Apêndice 2) é dada por,

$$
\left.\begin{array}{l}
\pi\left(\theta_{1}^{*} / \text { dados }\right) \alpha \theta_{1}^{-\left(r^{\prime}+1 / 2\right)} e^{-\hat{\beta}_{1}\left(a_{1}^{\left.*^{\prime}-r^{\prime} X_{1}\right)}\right.} \\
\frac{\exp \left\{-\theta_{i}^{*-1} \sum_{i=2}^{3} A_{i} e^{\left.-\left(z_{i}-z_{1}\right)+\dot{\beta}_{1}\left(X_{1}-x_{i}\right)\right\}}\right.}{\left\{\sum_{i=2}^{3} A_{i}\left(X_{1}-X_{i}\right)^{2} e^{\left.-\left(z_{i}-z_{1}\right)+\dot{\beta}_{1}\left(X_{1}-x_{i}\right)\right\}^{\frac{1}{2}}}\right.},
\end{array}\right\}
$$

onde $\theta_{1}^{*}>0$ e $\hat{\beta}_{1}$ maximiza $-\beta\left(a_{1}^{*^{\prime}}-r^{\prime} X_{1}\right)-\theta_{1}^{*-1} \sum_{i=2}^{3} A_{i} e^{-\left(Z_{i}-Z_{1}\right)+\beta_{1}\left(X_{1}-X_{i}\right)}$

Observe a equivalência entre (7.19) e (6.14).

Assim, mesmo sem a utilização do nível usual de estresse, $X_{1}$, podemos obter inferências sobre o tempo médio de sobrevivência, $\theta_{1}^{*}$. 


\section{Capítulo 8}

\section{Análise de Testes de Sobrevivência Acelerados com o Modelo de Eyring Generalizado $\ldots$}

Até o momento, consideramos experimentos acelerados de componentes submetidos a níveis de uma única variável física de estresse. Comumente encontramos situações práticas onde os componentes são submetidos a níveis de duas ou mais váriaveis estresse.

O modelo de Eyring generalizado (1.6), como visto na seção 1.2, é uma das relações estresse-resposta mais utilizadas em testes de sobrevivência acelerados de componentes que estão sob efeito de dois tipos de estresse, um termal e outro não-termal, por exemplo, voltagem e temperatura.

Neste capítulo, assumindo dados sob esquema de censura de Tipo II, apresentamos os resultados de uma Análise Bayesiana do modelo de Eyring generalizado, considerando inicialmente uma distribuição de sobrevivência Exponencial, e enfatizamos os problemas existentes em uma análise clássica usual deste modelo.

Em uma segunda etapa, considerando uma distribuição de Weibull para os 
1.mpos de sobrevivência, com parâmetro de forma comum para todas as combinações de níveis de estresse, mostramos que, assumindo o modelo de Eyring generalizado com dois parâmetros conhecidos, é possível analisá-lo da mesma forma que fizemos com a relação estresse-resposta geral (6.1).

\subsection{Formulação do Experimento Acelerado}

Considere o modelo de Eyring generalizado (1.6), dado por,

$$
\lambda_{i}=\alpha V_{i} \exp \left\{\dot{\gamma} U_{i}+\delta \frac{U_{i}}{k_{1} V_{i}},-\beta \frac{1}{k_{1} V_{i}}\right\} \therefore
$$

e assuma dados sob um esquema de censura de Tipo II.

Sob $k$ combinações aleatorizadas de níveis de estresse $U_{i}$ e $V_{i}, i=1,2, \ldots, k$, o experimento termina quando observamos $r_{i}$ falhas entre $j_{i}$ as $n_{i}$ observações na $i-$ ésima combinação de estresse.

Assim, com $n_{i}$ unidades em teste sob os níveis de estresse $U_{i}$ e $V_{i}$, temos, $r_{i}$ observações ordenadas não censuradas dadas por, $t_{i 1}<t_{i 2}<\ldots<t_{i r_{i}}$, e $n_{i}-r_{i}$ observações censuradas iguais a $t_{i r_{i}}, i=1,2, \ldots, k$.

\subsection{Procedimento usual de Análise do Modelo}

$\mathrm{Na}$ análise do modelo de Eyring generalizado é usual obter os estimadores de mínimos quadrados dos parâmetros do modelo linear, pois a obtenção dos estimadores de máxima verossimilhança requer a solução de quatro equações não-lineares simultâneas (ver por exemplo, Mann, Schaffer e Singpurwalla, 1974), o que de certa forma demanda um esforço computacional relativamente grande. 
Seja $\hat{\lambda}_{i}=r_{i} / A_{i}$, onde $A_{i}=\sum_{i=1}^{r_{i}} t_{i j}+\left(n_{i}-r_{i}\right) t_{i r_{i}}$, o estimador de máxima verossimilhança de $\lambda_{i}$ sob a i-ésima combinação de niveis de estresse $U_{i} \mathrm{e} V_{i}$, considerandose a f.d.p. dada $(e m(2.1))$ por,

$$
f\left(t, \lambda_{i}\right)=\lambda_{i} \exp \left\{-\lambda_{i} t\right\}
$$

Considerando um termo de erro multiplicativo, e o modelo $\hat{\lambda}_{i}=\lambda_{i} \xi_{i}, i=$ $1,2, \ldots, k, \operatorname{temos}(\operatorname{de}(8.1))$,

$$
\log \hat{\lambda}_{i}=\log \alpha+\log V_{i}+\gamma U_{i}+\delta \frac{U_{i}}{k_{1} V_{i}}-\beta \frac{1}{k_{1} V_{i}}+\log \xi_{i}
$$

Definindo,

$$
\begin{aligned}
\underline{\alpha}^{\prime} & =(\log \alpha, \gamma, \delta,-\beta) \\
\underline{\xi}^{\prime} & =\left(\log \xi_{1}, \log \xi_{2}, \ldots, \log \xi_{k}\right), \\
\underline{Y}^{\prime} & =\left(\log \left(\hat{\lambda}_{1} / V_{1}\right), \log \left(\left(\hat{\lambda}_{2} / V_{2}\right), \ldots, \log \left(\hat{\lambda}_{k} / V_{k}\right)\right)\right.
\end{aligned}
$$

$$
\mathbf{X}=\left[\begin{array}{cccc}
1 & U_{1} & U_{1} / k_{1} V_{1} & \left(k_{1} V_{1}\right)^{-1} \\
1 & U_{2} & U_{2} / k_{1} V_{2} & \left(k_{1} V_{2}\right)^{-1} \\
\vdots & \vdots & \vdots & \vdots \\
\vdots & \vdots & \vdots & \vdots \\
1 & U_{k} & U_{k} / k_{1} V_{k} & \left(k_{1} V_{k}\right)^{-1}
\end{array}\right]
$$

o modelo (8.2) pode ser escrito na forma matricial,

$$
Y=\mathrm{X} \underline{\alpha}+\underline{\xi} .
$$


O melhor est imador linear não-viciado para o vetor de parâmetros $\underline{\alpha}$ pode ser obtido se as condições necessárias para usarmos o Teorema de Gauss - Markoff são satisfeitas, isto é, $E(\xi)=0$ e $E\left(\underline{\xi \xi^{\prime}}\right)=\delta^{2} I$, onde $\sigma^{2}$ é constante e II é a matriz identidade. Dessa forma, se a distribuição do vetor $\underline{Y}$ é Normal multivariada, os limites de confiança para o vetor de parâmetros $\underline{\alpha}$ podem ser obtidos com facilidade (ver por exemplo, Seber, 1977).

Singpurwalla e Goldschen (1974) mostram, através de similações de Monte -Carlo, que as condições necessárias para usarmos o Teorema de Gauus-Markoff é que $\log \left(\hat{\lambda}_{i}\right)$ tenha distribuição Normal, e que $r_{i}=r$ para todo $i$, isto é, que o número de falhas seja o mesmo em todas as combinações de níveis de estresse $U_{i}$ e $V_{i} i=1,2, \ldots, k$ (ver por exemplo, Mann, Schaffer e Singapurwalla, 1974).

Portanto, assumindo $r_{i}=r, i=1,2, \ldots, k$ e $\log \hat{\lambda}_{i}$ com distribuição Normal, os estimadores de mínimos quadrados dos parâmetros do modelo são dados por,

$$
\begin{aligned}
& \underline{\hat{\alpha}}^{\prime}=\left(\mathrm{X}^{\prime} \mathbf{X}\right)^{-1} \mathrm{X} \underline{Y}, \\
& \underline{\hat{\alpha}} \stackrel{a}{\sim} N\left(\underline{\alpha} ; \sigma^{2}\left(\mathrm{X}^{\prime} \mathbf{X}\right)^{-1}\right),
\end{aligned}
$$

onde $\underline{\alpha}^{\prime}=\left(\hat{\alpha}_{1}, \hat{\alpha}_{2}, \hat{\alpha}_{3}, \hat{\alpha}_{4}\right), \alpha_{1}=\log \alpha, \alpha_{2}=\gamma, \alpha_{3}=\delta$ e $\alpha_{4}=-\beta$.

Devido a suposição de normalidade, os elementos de $\underline{\hat{\alpha}}$ são os estimadores de máxima verossimilhança dos elementos de $\underline{\alpha}$.

Com os estimadores $\hat{\alpha}_{1}, \hat{\alpha}_{2}, \hat{\alpha}_{3}, \dot{e} \hat{\alpha}_{4}$, observamos que,

$$
\hat{\lambda}_{i}=V_{i} \exp \left\{\hat{\alpha}_{1}+\hat{\alpha}_{2} U_{i}+\hat{\alpha}_{3} \frac{U_{i}}{k_{1} V_{i}}+\hat{\alpha}_{4} \frac{1}{k_{1} V_{i}}\right\}
$$


é um estimador assintoticamente não-viciado para $\lambda_{i}$ sob os níveis de estresse $U_{i} \mathrm{e}$ $V_{i}$, e um intervalo de confiaça $100\left(1-\alpha^{\prime}\right) \%$ para $\lambda_{i}$ é dado por,

$$
p\left[\hat{\lambda}_{i} \exp \left\{t_{\left(\alpha^{\prime} / 2\right)} J_{i}\right\} \leq \lambda_{i} \leq \hat{\lambda}_{i} \exp \left\{-t_{\left(\alpha^{\prime} / 2\right)} J_{i}\right\}\right]=1-\alpha^{\prime}
$$

onde $J_{i}=\left\{S^{2}\left[1+X_{i}^{\prime}\left(\mathrm{X}^{\prime} \mathrm{X}\right)^{-1} X_{i}\right]\right\}^{1 / 2}, X_{i}^{\prime}=\left(1, U_{i}, \frac{U_{i}}{K_{1} V_{i}}, \frac{1}{K_{1} V_{i}}\right), S^{2}=$ $\left\{\underline{Y}^{\prime}\left[\mathrm{I}-\mathrm{X}\left(\mathrm{X}^{\prime} \mathrm{X}\right)^{-1} \mathrm{X}^{\prime}\right] \underline{Y}\right\} /(\mathrm{k}-4)$, e $t_{\alpha^{\prime} / 2}$ é o $100\left(\alpha^{\prime} / 2\right)$-ésimo percentil da distribuição $t$ de Student com $\mathrm{K}-4$ graus de liberdade (ver por exemplo, Mann, Schaffer e Singpurwalla, 1974).

Podemos notar que quando usamos esta metodologia de análise estamos considerando um modelo linear com $\log \left(\hat{\lambda}_{i}\right)$ em lugar dos dados de cada combinação de estresse $U_{i}$ e $V_{i}, i=1,2, \ldots, k$, isto é, toda a variabilidade dos dados em cada combinação de níveis de estresse não é considerada. Também, o modelo tem um termo de erro multiplicativo, o qual pode não ser apropriado.

Assim, temos motivação suficiente para desenvolvermos uma Análise Bayesiana do modelo de Eyring generalizado.

\subsection{Análise Bayesiana do Modelo de Eyring Ge- neralizado assumindo uma Distribuição Ex- ponencial para os tempos de Sobrevivência}

Nesta seção, um procedimento Bayesiano de análise do modelo de Eyring generalizado é determinado. Consideramos inicialmênte os parâmetros $\alpha, \beta$ e $\gamma$ desconhecidos, depois assumimos $\gamma$ e $\delta$ conhecidos e finalmente, consideramos uma análise Bayesiana assumindo $\beta, \gamma$ e $\delta$ conhecidos. 


\subsubsection{Uma Análise Bayesiana assumindo $\alpha, \beta, \gamma$ e $\delta$ desco- nhecidos}

Assumindo $k$ combinações aleatorizadas de níveis de estresse $U_{i}$ e $V_{i}$, uma distribuição de sobrevivência Exponencial e um esquema de censura de Tipo II, a função de verossimilhança para $\underline{\lambda}^{\prime}=\left(\lambda_{1}, \lambda_{2}, \ldots, \lambda_{k}\right)$ é dada (ver seção 2.2) por,

$$
L(\lambda)=\prod_{i=1}^{k} \lambda_{i}^{r_{i}} \exp \left\{-\lambda_{i} A_{i}\right\}
$$

Substituindo $\lambda_{i}$, dado em (8.1), na expressão (8.7), obtemos a função de verossimilhança para $\alpha, \beta, \gamma$ e $\delta$ dada por,

$$
L(\alpha, \beta, \gamma, \delta)=\alpha^{r}\left(\prod_{i=1}^{k} V_{i}^{r_{i}}\right) \exp \left\{\gamma a_{1}+\delta a_{2}-\beta a_{3}-\alpha \sum_{i=1}^{k} V_{i} A_{i} e^{-b_{i}}\right\}
$$

onde $r=\sum_{i=1}^{k} r_{i}, a_{1}=\sum_{i=1}^{k} r_{i} U_{i}, a_{2}=\frac{1}{k_{1}} \sum_{i=1}^{k} r_{i} U_{i} / V_{i}, a_{3}=\frac{1}{k_{1}} \sum_{i=1}^{k} r_{i} / V_{i}$ e $b_{i}=$ $\frac{\beta}{k_{1} V_{i}}-\gamma U_{i}-\frac{\delta U_{i}}{k_{1} V_{i}}$.

A matriz de informação de Fisher com respeito a $\alpha, \beta, \gamma$ e $\delta$ é dada por,

$$
I(\alpha, \beta, \gamma, \delta)=\left[\begin{array}{ccccc}
\frac{r}{\alpha^{2}} & -\frac{1}{k_{1} \alpha} \sum_{i=1}^{k} \frac{r_{i}}{V_{i}} & \frac{1}{\alpha} \sum_{i=1}^{k} r_{i} U_{i} & \frac{1}{k_{1} \alpha} \sum_{i=1}^{k} \frac{r_{i} U_{i}}{V_{i}} \\
& \frac{1}{k_{1}^{2}} \sum_{i=1}^{k} \frac{r_{i}}{V_{i}^{2}} & -\frac{1}{k_{1}} \sum_{i=1}^{k} \frac{r_{i} U_{i}}{V_{i}} & -\frac{1}{k_{1}^{2}} \sum_{i=1}^{k} \frac{r_{i} U_{i}}{V_{i}^{2}} \\
& & & \sum_{i=1}^{k} r_{i} U_{i}^{2} & \frac{1}{k_{1}} \sum_{i=1}^{k} \frac{r_{i} U_{i}^{2}}{V_{i}} \\
& & & \\
\text { simétrica } & \vdots & & \frac{1}{k_{1}^{2}} \sum_{i=1}^{k} \frac{r_{i} U_{i}^{2}}{V_{i}^{2}}
\end{array}\right]
$$

Considerando (8.9), a densidade a priori não-informativa de Jeffreys para $\alpha, \beta, \gamma, \delta$ é dada por, 


$$
\pi(\alpha, \beta, \gamma, \delta) \propto \frac{1}{\alpha}
$$

onde $\propto>0 \mathrm{e}-\infty<\beta, \gamma, \delta<\infty$.

A densidade a posteriori conjunta para $\alpha, \beta, \gamma$ e $\delta$ é dada por,

$$
\pi(\alpha, \beta, \gamma, \delta / \text { dados }) \propto \alpha^{r-1} \exp \left\{\gamma a_{1}+\delta a_{2}-\beta a_{3}-\alpha \sum_{i=1}^{k} V_{i} A_{i} e^{\gamma U_{i}+\delta \frac{v_{i}}{k_{1} v_{i}}-\beta \frac{1}{k_{1} v_{i}}}\right\}
$$

onde $\alpha>0 \mathrm{e}-\infty<\beta, \gamma, \delta<\infty$.

A densidade a posteriori conjunta marginal para $\beta, \gamma$ e $\delta$ é dada (de(8.11)) por,

$$
\pi(\beta, \gamma, \delta / \text { dados }) \propto \exp \left\{\gamma a_{1}+\delta a_{2}-\beta a_{3}\right\}\left(\sum_{i=1}^{k} V_{i} A_{i} e^{\gamma U_{i}+\delta \frac{v_{i}}{\boldsymbol{k}_{1} V_{i}}-\beta \frac{1}{k_{1} V_{i}}}\right)^{-\tau}
$$

onde $-\infty<\beta, \gamma, \delta<\infty$.

Usando o método de Laplace completamente exponencial (ver Apêndice 2), a densidade a posteriori conjunta marginal aproximada para $\gamma$ e $\delta$ é dada por,

$$
\pi(\gamma, \delta / \text { dados }) \propto \frac{\exp \left\{\gamma a_{1}+\delta a_{2}-\hat{\beta} a_{3}\right\}\left\{h_{\gamma, \delta}^{\prime \prime}(\hat{\beta})\right\}^{1 / 2}}{\left\{\sum_{i=1}^{k} V_{i} A_{i} e^{\left.\gamma U_{i}+\delta \frac{V_{i}}{k_{1} V_{i}}-\hat{\beta} \frac{1}{k_{1} v_{i}}\right\}^{r}}\right.}
$$

ondè $-\infty<\gamma, \delta<\infty, \hat{\beta}$ maximiza $-n h_{\gamma, \delta}(\beta)=-\beta a_{3}-r \log \left(\sum_{i=1}^{k} V_{i} A_{i} e^{-b_{i}}\right)$ para cada $\operatorname{par}(\gamma, \delta)$ fixo, $b_{i}=\frac{\beta}{k_{1} V_{i}}-\gamma U_{i}-\frac{\delta U_{i}}{k_{1} V_{1}} \mathrm{e}$

$$
-n h_{\gamma, \delta}^{\prime \prime}(\beta)=\frac{r}{k_{1}} \frac{\left(\sum_{i=1}^{k} \frac{A_{i}}{V_{i}} e^{-b_{i}} \sum_{i=1}^{k} V_{i} A_{i} e^{-b_{i}}\right)-\left(\sum_{i=1}^{k} A_{i} e^{-b_{i}}\right)^{2}}{\left(\sum_{i=1}^{k} V_{i} A_{i} e^{-b_{i}}\right)^{2}}
$$




\subsubsection{Tima Análise Bayesiana assumindo $\gamma$ e $\delta$ conhecidos}

Assumindo $\gamma$ e $\delta$ conhecidos, a função de verossimilhanç para $\alpha$ e $\beta$ é dada $(\operatorname{de}(8.8))$ por,

$$
L(\alpha, \beta) \propto \alpha^{r} \exp \left\{-\beta a_{3}-\alpha \sum_{i=1}^{k} V_{i} A_{i} e^{-b_{i}}\right\}
$$

onde $a_{3}$ e $b_{i}$ são dados em (8.8).

Considerando uma priori não-informativa para $\alpha$ e $\beta$, dada por, $\pi(\alpha, \beta) \propto$ $\alpha^{-1}, \alpha>0,-\infty \leq \beta \leq \infty$, a densidade a posteriori conjunta para $\beta$ é dada por,

$$
\pi(\alpha, \beta / \text { dados }) \propto \alpha^{r-1} \exp \left\{-\beta a_{3}-\alpha \sum_{i=1}^{k} V_{i} A_{i} e^{-b_{i}}\right\}
$$

onde $\alpha>0$ e $-\infty<\beta<\infty$.

A densidade a posteriori marginal para $\beta$ é dada por,

$$
\pi(\beta / \text { dados }) \propto e^{-\beta a_{3}}\left\{\sum_{i=1}^{k} V_{i} A_{i} e^{\gamma U_{i}+\delta \frac{V_{i}}{k_{1} v_{i}}-\beta \frac{\lambda}{k_{1} V_{i}}}\right\}^{-r}
$$

onde $-\infty<\beta<\infty$.

Como temos interesse em infêrencias sobre o tempo médio sobrevivência, $\theta_{1}$, sob os níveis usuais de estresse, $U_{1}$ e $V_{1}$, obtemos agora a densimade a posteriori para $\theta_{1}$.

Observe que a densidade a posteriori conjunta para $\alpha$ e $\beta(8.15)$ pode ser escrita na forma, 


$$
\pi(\alpha, \beta / \text { dados }) \propto \alpha^{r-1} \exp \left\{-\beta a_{3}-\alpha \sum_{i=1}^{k} V_{i} A_{i} e^{\bar{c}_{i}-\beta \frac{d}{k_{1} V_{i}}}\right\}
$$

onde $c_{i}=\gamma U_{i}+\delta \frac{U_{i}}{k_{1} V_{i}}, \alpha>0,-\infty<\beta<\infty$

Considerando a transformação $\psi_{1}=\exp \left\{\beta / k_{1} V_{1}\right\}$ e $\psi_{2}=\alpha V_{i} e^{c_{1}}$ (isto é, $\beta=k_{1} V_{1} \log \left(\psi_{1}\right)$ e $\left.\alpha=\psi_{2} /\left(V_{1} e^{c_{1}}\right)\right)$, a densidade a posteriori conjunta para $\psi_{1}$ e $\psi_{2}$ é dada por,

$$
\pi\left(\psi_{1}, \psi_{2} / \text { dados }\right) \propto \psi_{2}^{r-1} \psi_{1}^{-\left(a_{3} k_{1} V_{1}+1\right)} \exp \left\{-\psi_{2} e^{-c_{1}} \sum_{i=1}^{k} \frac{V_{i}}{V_{1}} A_{i} e^{c_{i}} \psi^{-V_{1} / V_{i}}\right\}
$$

onde $\psi_{1}, \psi_{2}>0$

Observando que $(\operatorname{de}(8.1))$ sob os níveis usuais de estresse, $\theta_{1}=1 / \lambda_{1}=$ $\psi_{1} / \psi_{2}$, e considerando a transformação $\theta_{1}=\psi_{1} / \psi_{2}$ e $\psi_{2}=\psi_{2}$ temos que a densidade a posteriori conjunta para $\theta_{1}$ e $\psi_{2}$ é dada por,

$$
\pi\left(\theta_{1}, \psi_{2} / \text { dados }\right) \propto \frac{\exp \left\{-\psi_{2} e^{-c_{1}} \sum_{i=1}^{k} \frac{V_{i}}{V_{1}} A_{i} e^{c_{i}} \psi_{2}^{-V_{1} / V_{i}} \theta_{1}^{-V_{1} / V_{i}}\right\}}{\psi_{2}^{a_{3} k_{1} V_{1}-\tau+1} \theta_{1}^{a_{3} k_{1} V_{1}+1}}
$$

onde $\psi_{1}, \psi_{2}>0$

Utilizando o método de aproximação de Laplace completamente exponencial (ver Apêndice 2), a densidade a posteriori marginal aproximada para $\theta_{1}$ é dada por,

$$
=\pi\left(\theta_{1} / \text { dados }\right) \propto \frac{\exp \left\{-e^{-c_{1}} \sum_{i=1}^{k} \frac{V_{i}}{V_{1}} A_{i} e^{c_{i}} \hat{\psi}_{2}^{\left(1-\frac{v_{1}}{V_{i}}\right)} \theta_{1}^{-V_{1} / V_{i}}\right\}}{\theta_{1}^{a_{3} k_{1} V_{1}+1} \hat{\psi}_{2}^{a_{3} k_{1} V_{1}-r+1}\left\{h_{\theta_{1}}^{\prime \prime}\left(\hat{\psi}_{2}\right)\right\}^{1 / 2}}
$$


onde $\vartheta_{1}>0, \hat{\psi}_{2}$ maximiza $-n h_{\theta_{1}}\left(\psi_{2}\right)=-\left(a_{3} k_{1} V_{1}-r+1\right) \log \psi_{2}-$ $e^{-c_{1}} \sum_{i=1}^{k} \frac{V_{i}}{V_{1}} A_{i} e^{c_{i}} \psi_{2}^{\left(1-\frac{v_{1}}{v_{i}}\right)} \theta_{1}^{-V_{1} / V_{i}}, \mathrm{e}$

$$
-n h_{\theta_{1}}^{\prime \prime}\left(\psi_{2}\right)=\frac{a_{3} k_{1} V_{1}-r+1}{\psi_{2}^{2}}+e^{-c_{1}} \sum_{i=1}^{k} A_{1} e^{c_{i}}\left(1-\frac{V_{1}}{V_{i}}\right) \psi_{2}^{-\left(1+\frac{V_{1}}{V_{i}}\right)} \theta_{1}^{-\frac{V_{1}}{V_{i}}}
$$

Podemos observar que poderiamos ter obtido a densidade a posteriori marginal para $\theta_{1}$ considerando somente a transformação $\theta_{1}=\exp \left\{\beta / k_{1} V_{1}\right\} / \alpha V_{1} e^{c_{1}} \mathrm{e}$ $\beta=\beta$.

Também, até o momento, nada discutimos sobre a precisão das densidadẹs a posteriori aproximadas pelo método de Laplace completamente exponencial. Neste contexto, como a densidade a priori não-informativa de Jeffreys para $\alpha, \beta, \gamma, \delta$ (ver(8.10)) não é localmente uniforme, eventuais aproximações de Laplace completamente exponenciais para as densidades a posteriori dos parâmetros envolvidos, em geral, não terão boa precisão (ver por exemplo, Achar e Smith, 1990d).

O mesmo problema é detectado quando assumimos os parâmetros $\gamma$ e $\delta$ conhecidos, isto é, $\pi(\alpha, \beta) \propto \alpha^{-1}$ (não é localmente uniforme). Além disso, as densidades a priori não-informativas para os parâmetros transformados são dadas por, $\pi\left(\psi_{1}, \psi_{2}\right) \propto\left(\psi_{1}, \psi_{2}\right)^{-1}$ e $\pi\left(\theta_{1}, \psi_{2}\right) \propto\left(\theta_{1} \psi_{2}\right)^{-1}$, ambas não são constantes, sugerindo que a aproximação da densidade a posteriori marginal para $\theta_{1}(8.20)$, pelo método de Laplace completamente exponencial, não tem, em geral, boa precisão (ver por exemplo, Achcar, 1988, 1989).

Dessa forma, considerando a transformação $\phi_{1}=\log \theta_{1}$ e $\phi_{2}=\log \psi_{2}$, a densidade a priori para $\phi_{1}$ e $\phi_{2}$ é dada por,

$$
\pi\left(\phi_{1}, \phi_{2}\right) \propto \text { constante }
$$

onde $-\infty<\phi_{1}, \phi_{2:}<\infty$ (localmente uniforme). 
A densidade a posteriori conjunta para $\phi_{1}$ e $\phi_{2}$ é dada (de (8.19)) por,

$$
\pi\left(\phi_{1}, \phi_{2} / \text { dados }\right) \propto \frac{\exp \left\{-e^{-c_{1}} \sum_{i=1}^{k} \frac{V_{i}}{V_{1}} A_{i} e^{c_{i}-\phi_{1} \frac{V_{1}}{V_{i}}-\phi_{2} \frac{V_{1}}{V_{i}}}\right\}}{\exp \left\{\phi_{1} a_{3} k_{1} V_{1}+\phi_{2}\left(a_{3} k_{1} V_{1}-r+2\right)\right\}}
$$

onde $-\infty<\phi_{1}, \phi_{2}<\infty$.

Definindo $-n h_{\phi_{1}}\left(\phi_{2}\right)=-\phi_{2}\left(a_{3} k_{1} V_{1}-r+2\right)-e^{-c_{1}} \sum_{i=1}^{k} \frac{V_{i}}{V_{1}} A_{i} e^{c_{i}-\phi_{1}} \frac{v_{1}}{v_{i}}-\phi_{2} \frac{v_{1}}{v_{i}}$, a densidade a posteriori marginal para $\phi_{1}=\log \theta_{1}$ aproximada pelo método de Laplace completamente exponencial (ver Apêndice 2) é dada por,



onde $-\infty<\phi_{1}<\infty$ e $\hat{\phi}_{2}$ maximiza $-n h_{\phi_{1}}\left(\phi_{2}\right)$.

Observe que nesta parametrização $\left(\phi_{1}\right.$ e $\left.\phi_{2}\right)$, a densidade a posteriori aproximada (8.23) tem, em geral, boa precisão $\left(\pi\left(\phi_{1}, \phi_{2}\right)\right.$ é localmente uniforme).

\subsubsection{Uma Análise Bayesiana assumindo $\beta, \gamma$ e $\delta$ conheci- dos}

Assumindo $\beta, \gamma$ e $\delta$ conhecidos, a função de verossimilhança para $\alpha$ é dada (de (8.8)) por,

$$
L(\alpha) \propto \alpha^{r} \exp \left\{-\alpha \sum_{i=1}^{k} V_{i} A_{i} e^{\gamma U_{i}^{i}+\delta \frac{U_{i}}{k_{1} V_{i}}-\beta \frac{3}{k_{1} V_{i}}}\right\} .
$$

Considerando uma densidade a priori não-informativa para $\alpha$ dada por, $\pi(\alpha) \propto \alpha^{-1}, \alpha>0$, a densidade a posteriori para $\alpha$ é dada por, 


$$
\pi(\alpha / \text { dados }) \propto \alpha^{r-1} \exp \left\{-\alpha \sum_{i=1}^{k} V_{i} A_{i} e^{\gamma U_{i}+\delta \frac{U_{i}}{k_{1} v_{i}}-\beta \frac{1}{k_{1} v_{i}}}\right\}
$$

onde $\alpha>0$.

A densidade a posteriori para $\theta_{1}=e^{b_{1}} /\left(\alpha V_{1}\right), b_{1}=\beta \frac{1}{k_{1} V_{1}}-\gamma U_{1}-\delta \frac{U_{1}}{k_{1} V_{1}}$, é dada por,

$$
\pi\left(\theta_{1} / \text { dados }\right)=\frac{B^{r}}{V_{1}^{r} \Gamma(r)} \theta_{1}^{-(r+1)} \exp \left\{-\frac{B}{\theta_{1} V_{1}}\right\}
$$

onde $\theta_{1}>0, B=e^{b_{1}} \sum_{i=1}^{k} V_{i} A_{i} e^{-b_{i}}$ e $b_{i}=\beta \frac{1}{k_{1} V_{i}}-\gamma U_{i}-\delta \frac{U_{i}}{k_{1} V_{i}}$

A moda da densidade a posteriori (8.26) é dada por,

$$
\tilde{\theta}_{1}^{\prime}=\frac{B}{V_{1}(r+1)}
$$

Observe que (de (8.26)) a densidade a posteriori para $\frac{2 B}{V_{1} \theta_{1}}$ é a densidade de uma distribuição Qui-Quadrado com $2 r$ graus de liberdade. Portanto, um intervalo HPD $100\left(1-\gamma^{\prime}\right) \%$, para o tempo médio de sobrevivência $\theta_{1}$ é dado por,

$$
\left(\frac{2 B}{V_{1} \chi_{2 r\left(1-\frac{\gamma^{\prime}}{2}\right)}^{2}} ; \frac{2 B}{V_{1} \chi_{2 r\left(\frac{\gamma}{2}\right)}^{2}}\right)
$$

onde $\chi_{2 r\left(\gamma^{\prime} / 2\right)}^{2}$ é 0 quantil da distribuição Quj-Quadrado dado por, $P\left(\chi_{2 r}^{2} \leq \chi_{2 r\left(\gamma^{\prime} / 2\right)}^{2}\right)=\gamma^{\prime} / 2$. 


\subsection{Análise Bayesiana do Modelo de Eyring Ge- neralizado assumindo uma Distribuição de Sobrevivência de Weibull}

Na seção anterior onde desenvolvemos um procedimento Bayesiano de análise do modelo de Eyring generalizado considerando uma distribuição Exponencial, em nenhum momento nos preocupamos em fazer relação entre esse modelo e as relações estresse-resposta que consideram uma única variável estresse (ver seção 1.2).

Considerando uma distribuição de Weibull para os tempos de sobrevivência, mostramos nesta seção que, assumindo o modelo de Eyring generalizado com dois parâmetros conhecidos, é possível analisa-lo, no enfoque Bayesiano, de forma análoga à análise da relação estresse-resposta geral (6.1) (ver Capítulo 6).

\subsubsection{Procedimento Bayesiano de Análise Assumindo to- dọs os Parâmetros Desconhecidos}

Considere $T^{*}$ a variável aleatória com distribuição de Weibull, com f. d. p. dada $(\operatorname{de}(6.2))$ por,

$$
f\left(t^{*}, \lambda^{*}, p\right)=\lambda^{*} p\left(\lambda^{*} t^{*}\right)^{p-1} \exp \left\{-\left(\lambda^{*} t^{*}\right)^{p}\right\}
$$

onde $\lambda^{*}=\alpha V \exp \left\{\gamma U+\delta \frac{U}{k_{1} V}-\beta \frac{1}{k_{1} V}\right\}(U$ é a variável não-termal e $V$ é à variável temperatura) e $p$ é comum para todas as combinações de níveis de estresse.

Assuma o modelo de Eyring generalizado escrito na forma,

$$
\lambda_{i}^{*}=V_{i} \exp \left\{\alpha^{*}+\gamma U_{i}+\delta \frac{U_{i}}{k_{1} V_{i}}-\beta \frac{1}{k_{1} V_{i}}\right\}
$$


onde $\boldsymbol{\alpha}^{* \cdot}=\log \alpha$ e dados sob um esquema de censura de Tipo II.

Então, sob $k$ combinações de niveis de estresse $U_{i}$ e $V_{i}$, a função de verossimilhança para $\alpha^{*}, \gamma, \delta, \beta$ e $p$ é dada por,

$$
\left.\begin{array}{c}
L\left(\alpha^{*}, \beta, \gamma, \delta, p\right)=p^{r}\left(\prod_{i=1}^{k} \prod_{j=1}^{r_{i}} t_{i j}^{*^{p-1}}\right)\left(\prod_{i=1}^{k} V_{i}^{p r_{i}}\right) \cdot \\
\cdot \exp \left\{\alpha^{*} p r+\gamma p a_{1}+\delta p a_{2}-\beta p a_{3}\right\} . \\
. \exp \left\{-e^{-\alpha^{*} p} \sum_{i=1}^{k} A_{i}(p) V_{i}^{p} e^{\gamma p U_{i}+\delta p \frac{v_{i}}{k_{1} v_{i}}-\beta p \frac{1}{k_{1} v_{i}}}\right\},
\end{array}\right\}
$$

onde $r=\sum_{i=1}^{k} r_{i}, a_{1}=\sum_{i=1}^{k} r_{i} U_{i}, a_{2}=\frac{1}{k_{1}} \sum_{i=1}^{k} \frac{r_{i} U_{i}}{V_{i}}, a_{3}=\frac{1}{k_{1}} \sum_{i=1}^{k} r_{i} / V_{i} e A_{i}(p)=$ $\sum_{j=1}^{r_{i}} t_{i j}^{*^{p}}+\left(n_{i}+r_{i}\right) t_{i r_{i}}^{*^{p}}$.

Observe que, $\operatorname{com} \alpha^{*}=\log \alpha$ e $p=1,(8.30)$ reduz-se à expressão (8.8).

A matriz de informação de Fisher com respeito a $\alpha^{*}, \gamma, \delta, \beta$ dado $p$ é dada por,

$$
I_{p}\left(\alpha^{*}, \beta, \gamma, \delta\right)=\left[\begin{array}{cccc}
p^{2} r & -\frac{p^{2}}{k_{1}} \sum_{i=1}^{k} \frac{r_{i}}{V_{i}} & p^{2} \sum_{i=1}^{k} r_{i} U_{i} & p^{2} \sum_{i=1}^{k} \frac{r_{i} U_{i}}{V_{i}} \\
& \frac{p^{2}}{k_{1}^{2}} \sum_{i=1}^{k} \frac{r_{i}}{V_{i}^{2}} & \frac{-p^{2}}{k_{1}} \sum_{i=1}^{k} \frac{r_{i} U_{i}}{V_{i}} & \frac{-p^{2}}{k_{1}^{2}} \sum_{i=1}^{k} \frac{r_{i} U_{i}}{V_{i}^{2}} \\
& & p^{2} \sum_{i=1}^{k} r_{i} U_{i}^{2} & \frac{p^{2}}{k_{1}} \sum_{i=1}^{k} \frac{r_{i} U_{i}^{2}}{V_{i}} \\
\text { simétrica } & & & \frac{p^{2}}{k_{1}^{2}} \sum_{i=1}^{k} \frac{r_{i} U_{i}^{2}}{V_{i}^{2}}
\end{array}\right] .
$$

A densidade a priori não-informativa para $\alpha^{*}, \gamma, \delta, \beta$ e $p$ pode ser escrita na forma,

$$
\pi\left(\alpha^{*}, \beta, \gamma, \delta, p\right)=\pi\left(\alpha^{*}, \gamma, \delta, \beta / p\right) \pi_{0}(p)
$$


- Usando a regra multiparamétrica de Jefreys (ver por exemplo, Box e Tiao, 1973)

$$
\begin{array}{rlc}
\pi\left(\alpha^{*}, \beta, \gamma, \delta / p\right) & \propto & \operatorname{det}\left\{I_{p}\left(\alpha^{*}, \beta, \gamma, \delta\right)\right\}^{1 / 2} \\
& \propto & p^{2},
\end{array}
$$

onde $p>0$ e $-\infty<\alpha^{*}, \gamma, \delta, \beta<\infty$.

Assumindo $\pi_{0}(p) \propto 1 / p, p>0$ (densidade a priori localmente uniforme para $\log p$ ), a densidade a priori não-informativa para $\alpha^{*}, \gamma, \delta, \beta$ e $p$, baseada na regra multiparamétrica de Jeffreys, é dada por,

$$
\pi\left(\alpha^{*}, \beta, \gamma, \delta, p\right) \propto p
$$

onde $p>0$ e $-\infty<\alpha^{*}, \gamma, \delta, \beta<\infty$.

Considerando a densidade a priori (8.33), a densidade a posteriori para $\alpha^{*}, \gamma, \delta, \beta$ e $p$ é dada por,

$$
\begin{gathered}
\pi\left(\alpha^{*}, \beta, \gamma, \delta, p / \text { dados }\right) \propto p^{r-1}\left(\prod_{i=1}^{k} \prod_{j=1}^{r_{i}} t_{i j}^{* p-1}\right)\left(\prod_{i=1}^{k} V_{i}^{p r_{i}}\right) \\
\exp \left\{\alpha^{*} p r+\gamma p a_{1}+\delta p a_{2}-\beta p a_{3}\right\} \\
\cdot \exp \left\{-e^{\alpha^{*} p} \sum_{i=1}^{k} A_{i}(p) U_{i}^{p} e^{\gamma p U_{i}+\delta p \frac{U_{i}}{k_{1} v_{i}}-\beta p \frac{1}{k_{1} v_{i}}}\right\}
\end{gathered}
$$

onde $p>0$ e $-\infty<\alpha^{*}, \beta, \gamma, \delta<\infty$.

Observe que, utilizando o método de Láplace para aproximaçãao de integrais (ver Apêndice 2), e com algum esforço algébrico, podemos obter a densidade a posteriori marginal para o parâmetro $p$. Além disso, considerar o modelo de Eyring generalizado na forma $(8.29)$ onde $\alpha^{*}=\log (\alpha)$ torna-se interessante a medida que 
no: 3 parametrização temos uma densidade a priori não-informativa nara $\alpha^{*}, \beta ; \gamma$ e $\delta$, lecalmente uniforme (ver (8.31) com $p=1$ ), onde, como visto nos capítulos anteriores, podemos ter maior precisão quando da utilização do método de aproximação de Laplace no cálculo de densidades a posteriori marginais.

\subsubsection{Procedimento Bayesiano de Análise assumindo $\gamma$ e $\delta$ conhecidos}

Assumindo $\gamma$ e $\delta$ conhecidos, a função de verossimilhança para $\alpha^{*}, \beta$ e $p$ é dada (de (8.30)) por,

$$
\left.\begin{array}{c}
L\left(\alpha^{*}, \beta, p\right) \propto p^{r}\left(\prod_{i=1}^{k} \prod_{j=1}^{k} t_{i=1}^{* p-1}\right)\left(\prod_{i=1}^{k} V_{i}^{p r_{i}}\right) \exp \left\{\alpha^{*} p r-\beta p a_{3}\right\} \\
\cdot \exp \left\{-e^{\alpha^{*} p} \sum_{i=1}^{k} A_{i}(p) V_{i}^{p} e^{c_{i}^{\prime}-\beta p \frac{1}{k_{1} v_{i}}}\right\}
\end{array}\right\}
$$

onde $r, a_{3}$ e $A_{i}(p)$ são dados em (8.30) e $c_{i}^{\prime}=\gamma p U_{i}+\delta p \frac{U_{i}}{k_{1} V_{i}}$.

Considerando uma densidade a priori não-informativa para $\alpha^{*}, \beta$ e $p$, dada por, $\pi\left(\alpha^{*}, \beta, p\right) \propto p, p>0 \mathrm{e}-\infty<\alpha^{*}, \beta<\infty$, a densidade a posteriori conjunta para esses três parâmetros é dada por,

$$
\begin{gathered}
\pi\left(\alpha^{*}, \beta, p / \text { dados }\right) \propto p^{r+1}\left(\prod_{i=1}^{k} \prod_{j=1}^{r_{i}} t_{i j}^{* p-1}\right)\left(\prod_{i=1}^{k} V_{i}^{p r_{i}}\right) \exp \left\{\alpha^{*} p r-\beta p a_{3}\right\} \\
\therefore \quad \exp \left\{-e^{\alpha^{*} p} \sum_{i=1}^{k} A_{i}(p) V_{i}^{p} e^{c_{i}^{\prime}-\beta p \frac{1}{k_{1} V_{i}}}\right\}
\end{gathered}
$$

onde $p>0,-\infty<\alpha^{*}, \beta<\infty$ e $c_{i}^{\prime}$ é dada em (8.35).

Podemos observar que, definindo adequadamente as variáveis estresse e os parâmetros na expressão (8.36), esta torna-se equivalente à expressão (6.6), isto é, definindo $\beta_{0}=-\alpha^{*}, \beta_{1}=\frac{\beta}{k_{1}}, X_{i}=\frac{1}{V_{i}}$ e $Z_{i}=\left(\log V_{i}+c_{i}^{\prime}\right)$, temos (8.36) igual à (6.6). 
Dessa forma, seria redundante finalizarmos a Análise Bayesiana do modelo de Eyring generalizado assumido $\gamma$ e $\delta$ conhecidos, pois, como esse modelo pode ser visto como caso particular do modelo geral (6.1), essa análise restringe-se à análise obtida no Capítulo 6. 


\section{Capítulo 9}

\section{Exemplos de Aplicação}

Após termos determinado nos capítulos anteriores uma metodologia Bayesiana de análise para testes de sobrevivência acelerados, é de grande interesse, a título de ilustração, desenvolvermos alguns exemplos de aplicação das técnicas obtidas. É o que faremos neste capítulo.

9.1 Exemplo 1

Neste primeiro exemplo de aplicação nosso objetivo é a comparação das análises Clássica e Bayesiana do modelo de Eyring com uma variável estresse desenvolvidas nos Capítulos 2 e 3, e a aplicação do procedimento de controle de qualidade obtido no Capítulo 4.

Os dados estão na Tabela 9.1, e foram gerados assumindo o modelo de Eyring com uma variável estresse (1.5) com $\alpha=-10$ e $\beta=5$, a Distribuição Exponencial (2.1) e um esquema de censura de tipo II. 


\begin{tabular}{rccccccccccc}
$\dddot{i}$ & $V_{i}^{*}$ & $n_{i}$ & $r_{i}$ & $\theta_{i}$ & $A_{i}$ & \multicolumn{4}{c}{ Dados não censurados } \\
\hline \hline 1 & 10 & 20 & 5 & 3631,55 & 18085,00 & 178 & 301 & 574 & 920 & 1007 \\
& & & & & & & & & & \\
2 & 15 & 20 & 2 & 6 & 2049,36 & 13182,00 & 101 & 115 & 147 & $609^{*}$ & 705 \\
& & & & & & 767 & & & & \\
3 & 20 & 20 & 7 & 1414,13 & 9907,03 & 89 & 96 & 106 & 246 & 315 \\
& & & & & & 347 & 622 & & & \\
4 & 25 & 20 & 9 & 1076,13 & 9646,02 & 95 & 98 & 131 & 221 & 236 \\
& & & & & & 283 & 377 & 561 & 637 & \\
5 & 30 & 20 & 10 & 867,37 & 8676,00 & 24 & 62 & 245 & 314 & 332 \\
& & & & & & 338 & 386 & 401 & 491 & 553 \\
6 & 35 & 20 & 12 & 725,97 & 8763,96 & 73 & 96 & 132 & 177 & 211 \\
& & & & & & 345 & 361 & 378 & 399 & 416 \\
& & & & & & 560 & 624 & & & \\
7 & 40 & 20 & 13 & 623,98 & 7837,96 & 13 & 16 & 52 & 52 & 148 \\
& & & & & & 229 & 255 & 288 & 317 & 359 \\
& & & & & & 560 & 581 & 621 & & \\
8 & 45 & 20 & 14 & 547,00 & 7466,06 & 17 & 21 & 32 & 52 & 68 \\
& & & & & & 87 & 112 & 197 & 366 & 384 \\
& & & & & & 395 & 490 & 611 & 662 & \\
9 & 50 & 20 & 15 & 486,96 & 7137,90 & 10 & 13 & 83 & 87 & 129 \\
& & & & & & 135 & 196 & 244 & 250 & 262 \\
& & & & & & 264 & 293 & 318 & 624 & 705 \\
10 & 55 & 20 & 18 & 438,59 & 8372,98 & 11 & 39 & 53 & 90 & 96 \\
& & & & & & 115 & 153 & 228 & 259 & 323 \\
& & & & & & 356 & 388 & 411 & 597 & 761 \\
& & & & & & 763 & 823 & 969 & & \\
\hline
\end{tabular}

$\left(^{*}\right)$ níveis da variável estresse temperatura.

Tabela 9.1: Dados gerados com $\alpha=-10$ e $\beta=5-$ tempos de sobrevivência dos componentes. 


\subsubsection{Verificação Gráfica da Adequabilidade do Modelo}

Um procedimento gráfico pode ser adotado para verificarmos a adequabilidade do modelo de Eyring com uma variável estresse (1.5).

Considere a expressão (2.6) que relaciona a taxa constante de falha, $\lambda, \mathrm{e}$ a variável estresse, $V$, sob o i-ésimo nível de estresse dada por, $\lambda_{i}=V_{i} \exp \{\alpha-$ $\left.\beta / V_{i}\right\}, i=1 ; 2, \ldots, k$, isto é,

$$
\log \left(\frac{\lambda_{i}}{V_{i}}\right)=\alpha-\beta \frac{1}{V_{i}}
$$

Considerando os dados da Tabela 9.1 e os estimadores de máxima verossimilhança $\hat{\lambda}_{i}=\frac{r_{i}}{A_{i}}, i=1,2, \ldots, k$ (ver por exemplo, Lawless, 1982), podemos construir um gráfico de $\log \left(\frac{\bar{\lambda}_{i}}{V_{i}}\right)$ versus $\frac{1}{V_{i}}$, e verificar a existência ou não de uma relação linear aproximada. Caso exista, dizemos que o modelo de Eyring com uma variável estresse é válido para a amostra em questão.

\begin{tabular}{cccc}
\hline$\hat{\lambda}_{i}$ & $V_{i}$ & $\log \left(\frac{\hat{\lambda}_{i}}{V_{i}}\right)$ & $\frac{1}{V_{i}}$ \\
\hline \hline 0,0002765 & 10 & $-10,4960$ & 0,1000 \\
0,0004552 & 15 & $-10,4029$ & 0,0667 \\
0,0007066 & 20 & $-10,2508$ & 0,0500 \\
0,0009330 & 25 & $-10,1960$ & 0,0400 \\
0,0011526 & 30 & $-10,1669$ & 0,0333 \\
0,0013692 & 35 & $-10,1488$ & 0,0286 \\
0,0016586 & 40 & $-10,0907$ & 0,0250 \\
0,0018752 & 45 & $-10,0857$ & 0,0222 \\
0,0021015 & 50 & $-10,0771$ & 0,0200 \\
0,0021498 & 55 & $-10,1497$ & 0,0182 \\
\hline Tabela 9.2: Valores de $\hat{\lambda}_{i}, V_{i}, \log \left(\frac{\hat{\lambda}_{i}}{V_{i}}\right) \mathrm{e} \frac{1}{V_{i}}$.
\end{tabular}

Dessa forma, obtemos a Tabela 9.2 dos valores de $\log \left(\frac{\hat{\lambda}_{i}}{V_{i}}\right)$ e $\frac{1}{V_{i}}$ e construímos (ver Figura 9.1) o gráfico de $\log \left(\frac{\hat{\lambda}_{i}}{V_{i}}\right)$ versus $\frac{1}{v_{i}}$. 
Podemos observar na Figura 9.1 a existência de uma relação linear aproximada, implicando na adequabilidade do conjunto de dados gerados (ver Tabela 9.1) ao modelo de Eyring com uma variável estresse.

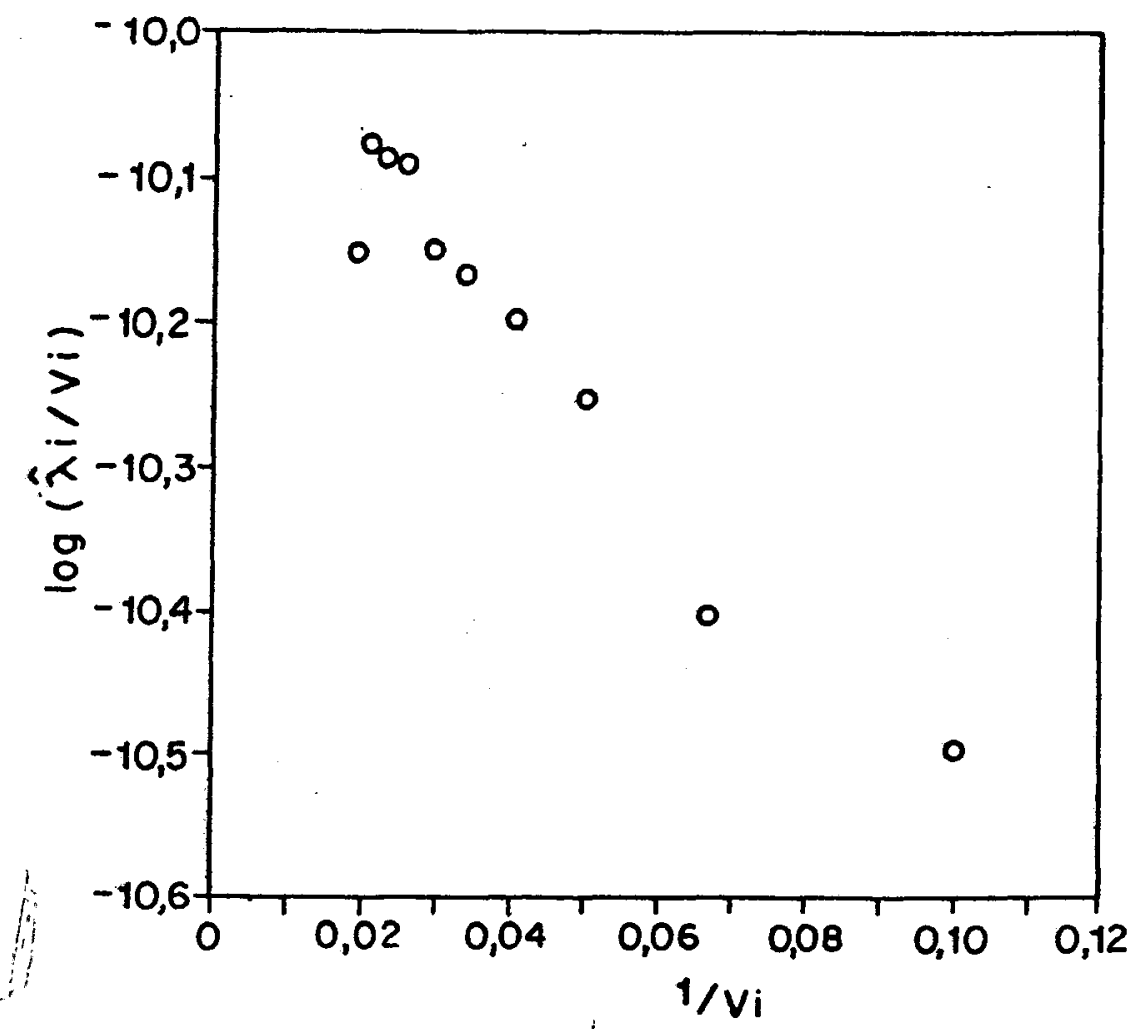

Figura 9.1: Gráfico de $\log \left(\frac{\hat{\lambda}_{i}}{V_{i}}\right)$ versus $\frac{1}{V_{i}}$.

\subsubsection{Análise Clássica do Problema}

Nesta seção, aplicamos, aos dados da Tabela 9.1, a metodologia de análise clássica, baseada na aproximação Normal assintótica dos estimadores de máxima verossimilhança dos parâmetros envolvidos (ver Capítulo 2).

Considerando os dados da Tabela 9.1 e as expressões (2.11) e (2.12), obtemos, numericamente, os estimadores de máxima verossimilhança para $\alpha$ e $\beta$ dados por, $\hat{\alpha}=-9,9971$ e $\hat{\beta}=5,2052$. 
Como $r=109, a_{1}=3,5496$ e $a_{2}=0,1565$, a inversa da matriz de informação de Fisher para $\alpha$ e $\beta$ é dada (de (2.19)) por,

$$
I^{-1}(\alpha, \beta)=\left[\begin{array}{cc}
0,0351 & 0,7961 \\
0,7961 & 24,4460
\end{array}\right]
$$

e os intervalos de confiança $95 \%$ para $\alpha$ e $\beta$, considerando-se a aproximação assintótica dos estimadores de máxima verossimilhança $\hat{\alpha}$ e $\hat{\beta}$ (2.13), são dados por, $-10,3643 \leq \alpha \leq-9,6299$ e $-4,4856 \leq \beta \leq 14,8960$.

Usualmente, os pesquisadores industriais têm interesse em inferências sobre o tempo médio de sobrevivência, $\theta_{1}$, sob o nível usual de estresse, $V_{1}$.

O estimador de máxima verossimilhança para $\theta_{1}=\frac{e \frac{\beta}{10}}{10 e^{\alpha}}$, considerandose os estimadores de máxima verossimilhança $\hat{\alpha}$ e $\hat{\beta}$, obtidos acima, é dado por, $\hat{\theta}_{1}=3696,2453$.

A variância assintótica para $\theta_{1}$ é dada (de (2.21)) por, $\hat{\sigma}_{\theta_{1}}^{2}=1.645 .609,0910$, e um intervalo de confiança $95 \%$ para $\theta_{1}$, considerando-se a aproximação Normal assintótica (2.20), obtida através da utilização do método "Delta" (ver Apêndice 1), é dado por, $1181,9320 \leq \theta_{1} \leq 6210,5590$.

Como visto no Capítulo 2, a aproximação assintótica (2.20), em geral, não tem boa precisão. Assim consideramos a reparametrização $\delta=\log \theta_{1}$ (ver sub -seção 2.6.3), onde temos uma melhor aproximação Normal do estimador de máxima verossimilhança de $\delta$.

O estimador de máxima verossimilhança para $\delta$ é dado (de (2.47)) por, $\hat{\delta}=8,215074$, a variância assintótica de $\delta$ é dada (de (2.51)) por, $\hat{\sigma}_{\delta}^{2}=0,120449$, e um intervalo de confiança $95 \%$ aproximado para $\theta_{1}$ é dado por, $1872,0699 \leq \theta_{1} \leq$ 7297,5941 . 


\subsubsection{Uma Solução Bayesiana para o Problema}

Como visto no Capítulo 3, em inferência Bayesiana, inferências sobre os parâmetros de interesse são tipicamente baseadas nas suas densidades a posteriori.

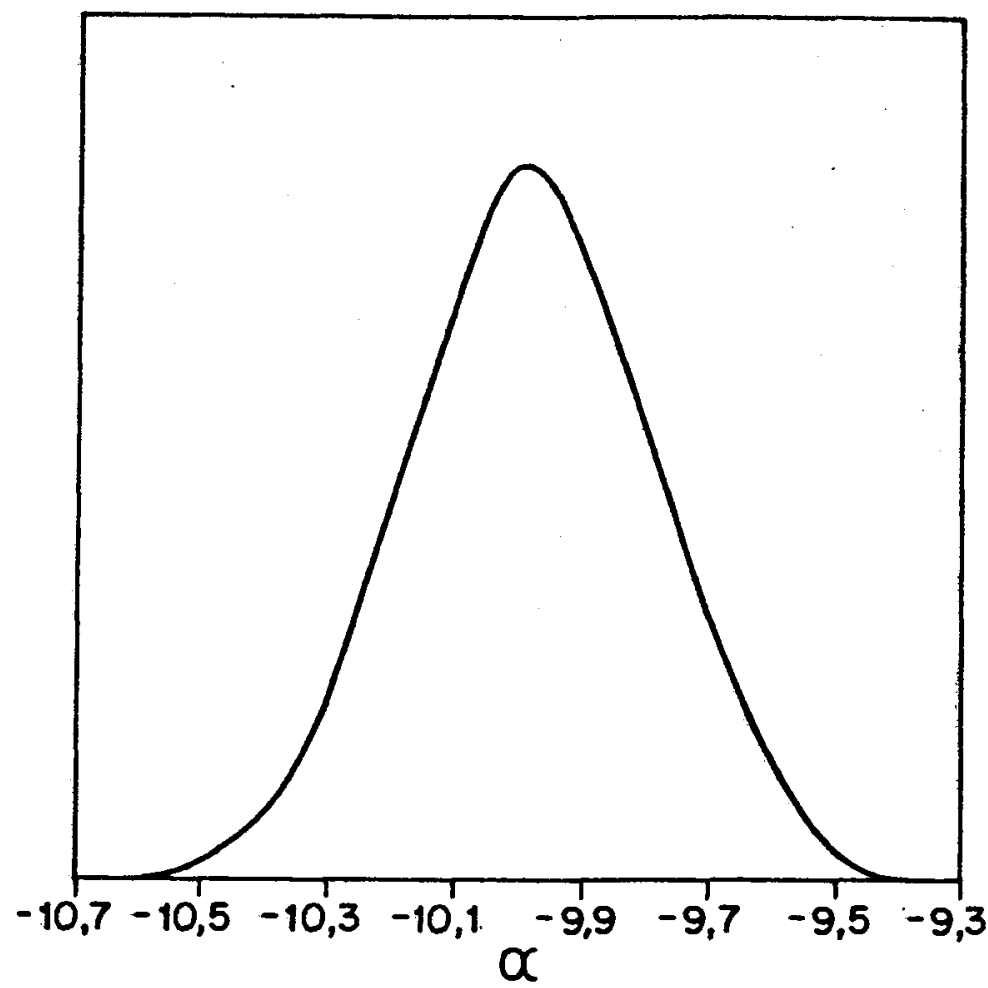

Figura 9.2: Densidade a posteriori marginal para $\alpha$.

Para inferirmos sobre os parâmetros de $\alpha$ e $\beta$, considerando o conjunto de dados da Tabela 9.1, construímos o gráfico da densidade a posteriori marginal aproximada para $\alpha$ (3.6), obtida pelo método de aproximação de Laplace completamente exponencial, e o gráfico da densidade a posteriori marginal para $\beta$ (3.4) (ver Figuras 9.2 e 9.3). As modas dessas densidades a posteriori são dadas por, $\tilde{\alpha}=-9,9998$ e $\tilde{\beta}=5,2040$. Os intervalos HPD aproximados $95 \%$ para $\alpha$ e $\beta$ são dados por, $-10,3807 \leq \alpha \leq-9,5887$ e $-4,0197 \leq \beta \leq 15,8504$ (ver no Apêndice 
4 a construção de Intervalos HPD).

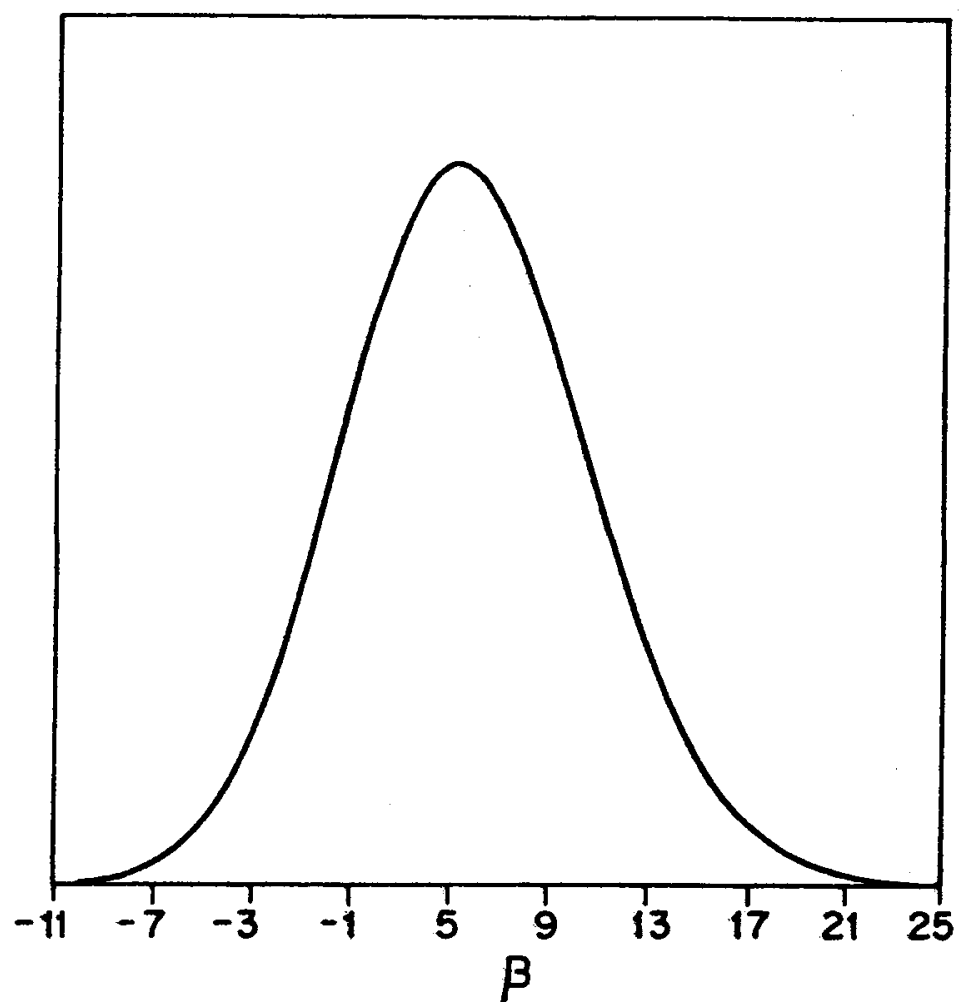

Figura 9.3: Densidade a posteriori marginal para $\beta$.

Na Figura 9.4 temos o gráfico da densidade a posteriori marginal para $\theta_{1}$ (3.9), aproximada pelo método de Laplace, cuja moda a posteriori é dada por $\tilde{\theta}_{1}=3704,00$. Um intervalo HPD aproximado $95 \%$ para $\theta_{1}$ é dado por, $1981,2549 \leq$ $\theta_{1} \leq 7899,6863$.

Alternativamente, um intervalo HPD aproximado para $\theta_{1}$ pode ser obtido considerando-se a transformação $\delta=\log \theta_{1}$. Nesta parametrização, como visto no Capítulo 3, temos uma boa precisão da aproximação de Laplace para a densidade a posteriori marginal para $\delta$ dada em (3.14). Assim construímos a Figura 9.5 do gráfico da densidade a posteriori marginal para $\delta$. A moda desta densidade a posteriori é dada por $\tilde{\delta}=8,217169\left(\tilde{\theta}_{1}=3704,0015\right)$. Um intervalo HPD aproximado $95 \%$ para $\delta$ é dado por, $7,6339 \leq \delta \leq 8,9429$, isto é, um intervalo HPD aproximado $95 \%$ para 
$\theta_{1}$ é dado por, $2067,0960 \leq \theta_{1} \leq 7653,3596$.

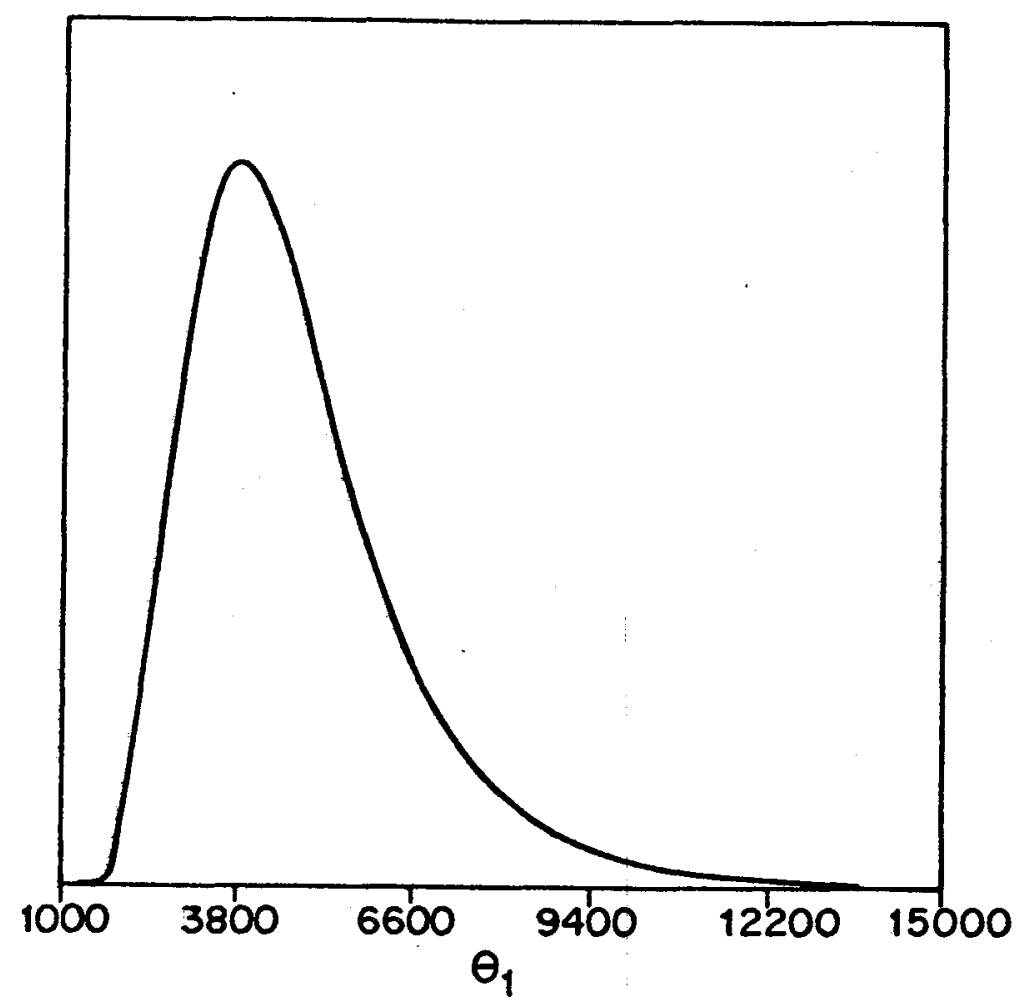

Figura 9.4: Densidade a posteriori marginal para $\theta_{1}$.

\subsubsection{Comparação entre as Estimativas Pontuais}

$\mathrm{Na}$ Tabela 9.3 temos os estimadores de máxima verossimilhança para $\alpha \mathrm{e}$ $\beta\left(\operatorname{ver}(2.11)\right.$ e (2.12), respectivamente) e $\hat{\theta}_{1}=e^{\beta / 10} / 10 e^{\hat{\alpha}}$ e as modas das densidades a posteriori para $\alpha$ e $\beta$ e $\theta_{1}$ (ver (3.6), (3.4) e (3.9), respectivamente).

\begin{tabular}{cccc}
\hline \multicolumn{4}{c}{$\begin{array}{c}\text { Estimador } \\
\text { de Máxima }\end{array}$} \\
Parâmetros & Valor para Geração & Verossimilhança & Moda a Posteriori \\
\hline \hline$\alpha$ & -10 & $\hat{\alpha}=-9,9971$ & $\tilde{\alpha}=-9,9998$ \\
$\beta$ & 5 & $\hat{\beta}=5,2052$ & $\tilde{\beta}=5,2040$ \\
$\theta_{1}$ & 3631,55 & $\hat{\theta}_{1}=3696,2453$ & $\tilde{\theta}_{1}=3704,0000$ \\
\hline
\end{tabular}

Tabela 9.3: Estimadores de máxima verossimilhança e modas a posteriori para os parâmetros $\alpha, \beta$ e $\theta_{1}$. 
Observamos que as estimativas pontuais para os parâmetros são muito próximas.

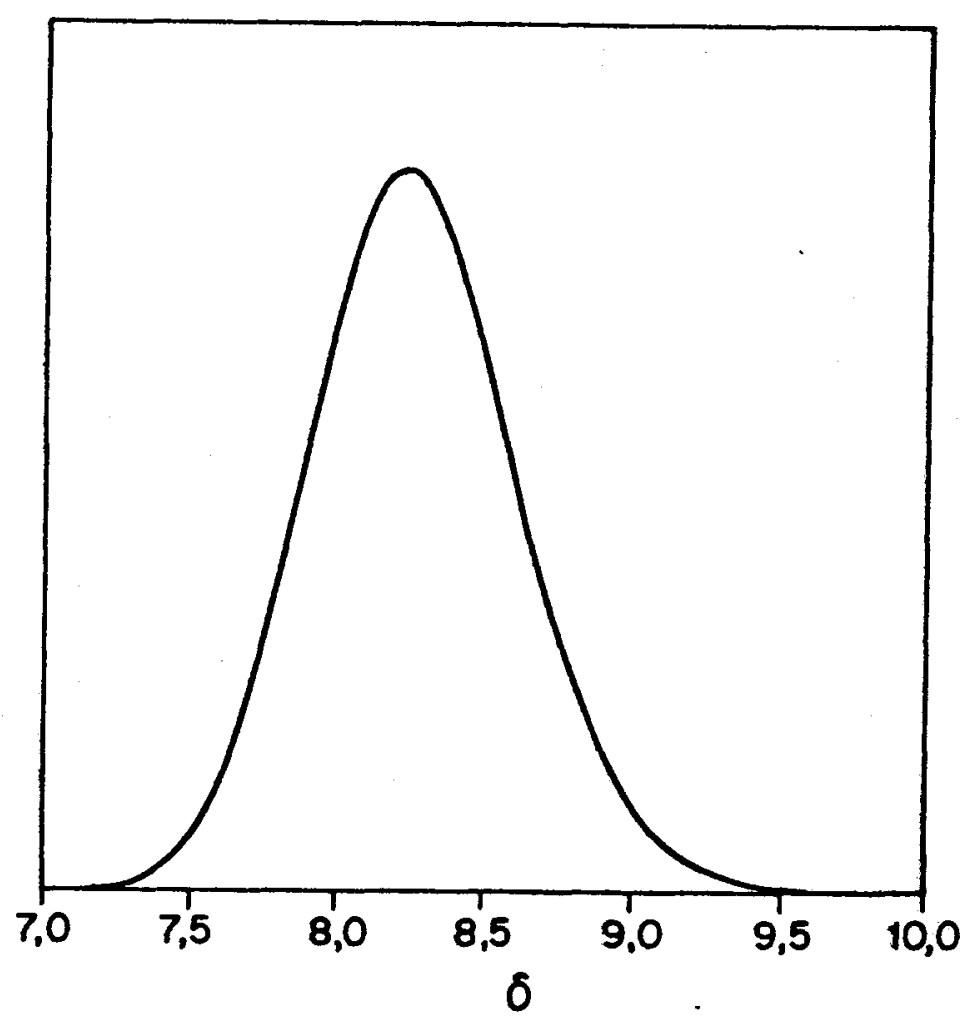

Figura 9.5: Densidade a posteriori marginal para $\delta=\log \theta_{1}$.

\subsubsection{Comparação entre as Estimativas por Intervalo}

Para compararmos as estimativas por intervalo obtidas, pelo método clássico de estimação (baseadas na aproximação Normal assintótica dos estimadores de máxima verossimilhança dos parâmetros envolvidos) e pẹla análise Bayesiana do problema, usamos a estatística da razão de verossimilhança assintótica, a qual é invariante sob parametrizações (ver por exemplo, Bickel e Doksum, 1977). Intervalos de confiança baseados nesta estatística podem ser obtidos graficamente via teoria de verossimilhança (ver por exemplo, Kalbfleisch, 1985; Ap̣êndice 3).

Considerando a função de verossimilhança para $\alpha$ e $\beta$ (2.8) obtemos as 
fun ^ ヘ̃os de verossimilhança relativas maximizadas para $\alpha$ e $\beta, r_{\beta}(\alpha)$ e $r_{\alpha}(\beta)$, respectivamente, e pelos gráficos de $\alpha$ versus $r_{\beta}(\alpha)$ e de $\beta$ versus $r_{\alpha}(\beta)$, determinamos os intervalos de confiança aproximados $95 \%$ para $\alpha$ e $\beta$ dados por, $-10,4863 \leq \alpha \leq$ $-9,6231$ e $-3,6331 \leq \beta \leq 15,6763$, como descrito no Apêndice 3 .

Analogamente, considerando $\ell\left(\theta_{1}, \beta\right)$ (dada em (2.24)), obtemos $r_{\beta}\left(\theta_{1}\right)$, e um intervalo de confiança aproximado $95 \%$ para $\theta_{1}$ é dado porr ,2055, $5556 \leq \theta_{1} \leq$ 7800,0000 .

\begin{tabular}{|c|c|}
\hline $\begin{array}{c}\text { Normalidade Assintótica } \\
\text { de } \hat{\alpha} \text { e } \hat{\beta}\end{array}$ & $\begin{array}{l}\text { Normalidade Assintótica } \\
\text { de } \hat{\theta}_{1} \text { (método "Delta") }\end{array}$ \\
\hline $\begin{array}{c}(-10,364 \leq \alpha \leq-9,630) \\
(-4,486 \leq \beta \leq 14,896)\end{array}$ & $\left(1181,932 \leq \theta_{1} \leq 6210,559\right)$ \\
\hline $\begin{array}{l}\text { Estatítica da Razão } \\
\text { Parametrização } \alpha \text { e } \beta\end{array}$ & $\begin{array}{c}\text { erossimilhança Assintótica } \\
\text { Parametrização } \theta_{1} \text { e } \beta\end{array}$ \\
\hline $\begin{array}{l}(-10,486 \leq \alpha \leq-9,623) \\
(-3,633 \leq \beta \leq 15,676)\end{array}$ & $\left(2055,556 \leq \theta_{1} \leq 7800,00\right)$ \\
\hline $\begin{array}{r}\text { Intervalos HPD Baseados } \\
\beta \text { e } \delta=\log \theta_{1} \text { dac }\end{array}$ & $\begin{array}{l}\text { Densidades a Posteriori para } \alpha \\
\mathrm{m}(3.6),(3.4) \text { e (3.14). }\end{array}$ \\
\hline $\begin{array}{c}(-10,357 \leq \alpha \leq-9,605) \\
(-3,588 \leq \beta \leq 15,984)\end{array}$ & $\left(2067,096 \leq \theta_{1} \leq 7653,036\right)$ \\
\hline
\end{tabular}

Tabela 9.4: Intervalos de confiança e Intervalos HPD 95\% para $\alpha, \beta$ e $\theta_{1}$.

Para facilitar a comparação construimos a Tabela 9.4 onde temos alguns intervalos de confiança e intervalos HPD para $\alpha, \beta$ e $\theta_{1}$.

Observamos (ver Tabela 9.4) que todos os intervasos para $\alpha$ e $\beta$ são aproximadamente iguais, porém, tal fato não ocorre para $\theta_{1}$. O intervalo HPD aproximado $95 \%$ para $\theta_{1}$ obtido através da densidade a posteriori para $\delta=\log \theta_{1}$ é muito próximo do intervalo de confiança $95 \%$ para $\theta_{1}$ obtido via estatística da razão de verossimilhança assintótica. Já o intervalo de confiança $95 \%$ para $\theta_{1}$ obtido pelo 
método "Delta" fornece um resultado bem diferente (como mencionado do Capítulo 2 , o pesquisador deve ter muito cuidado em utilizar esse método neste caso, pois a aproximação Normal, em geral, não é precisa).

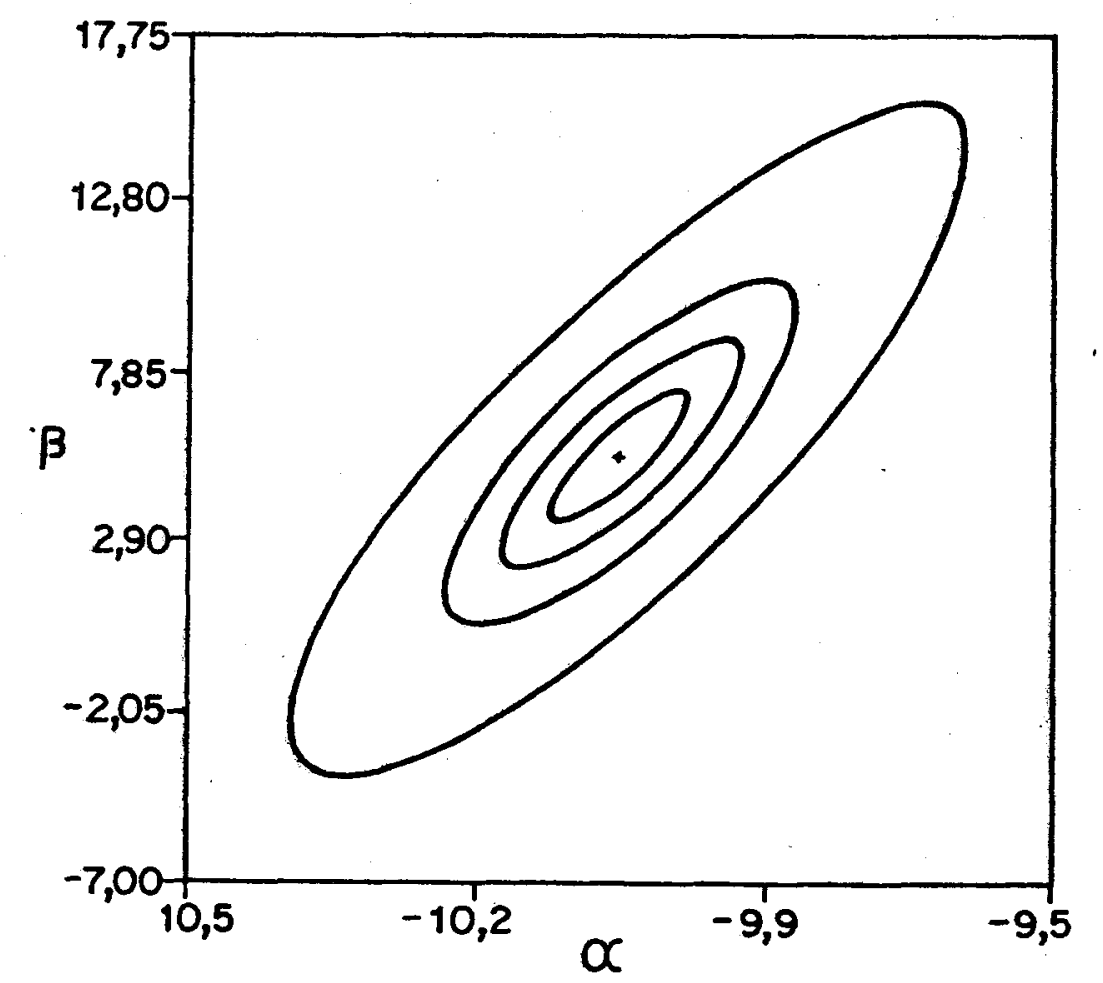

Figura 9.6: Contornos da função de verossimilhança para $\alpha$ e $\beta$.

Tal fato é ilustrado graficamente. Na Figura 9.6 temos o gráfico dos contornos da função de verossimilhança para $\alpha$ e $\beta$ (2.7). Observe a existência de uma boa forma elíptica, isto é, boa normalidade para os estimadores de máxima verossimilhança para $\alpha$ e $\beta$. Porém, considerando o gráfico dos contornos da função de verossimilhança para $\theta_{1}$ e $\beta$ (2.23) (ver Figura 9.7) notamos que não apresenta uma boa forma elíptica, sugerindo que não é precisa a aproximação Normal assintótica 
para a funñão de verossimilhança para $\theta_{1}$ e $\beta$.

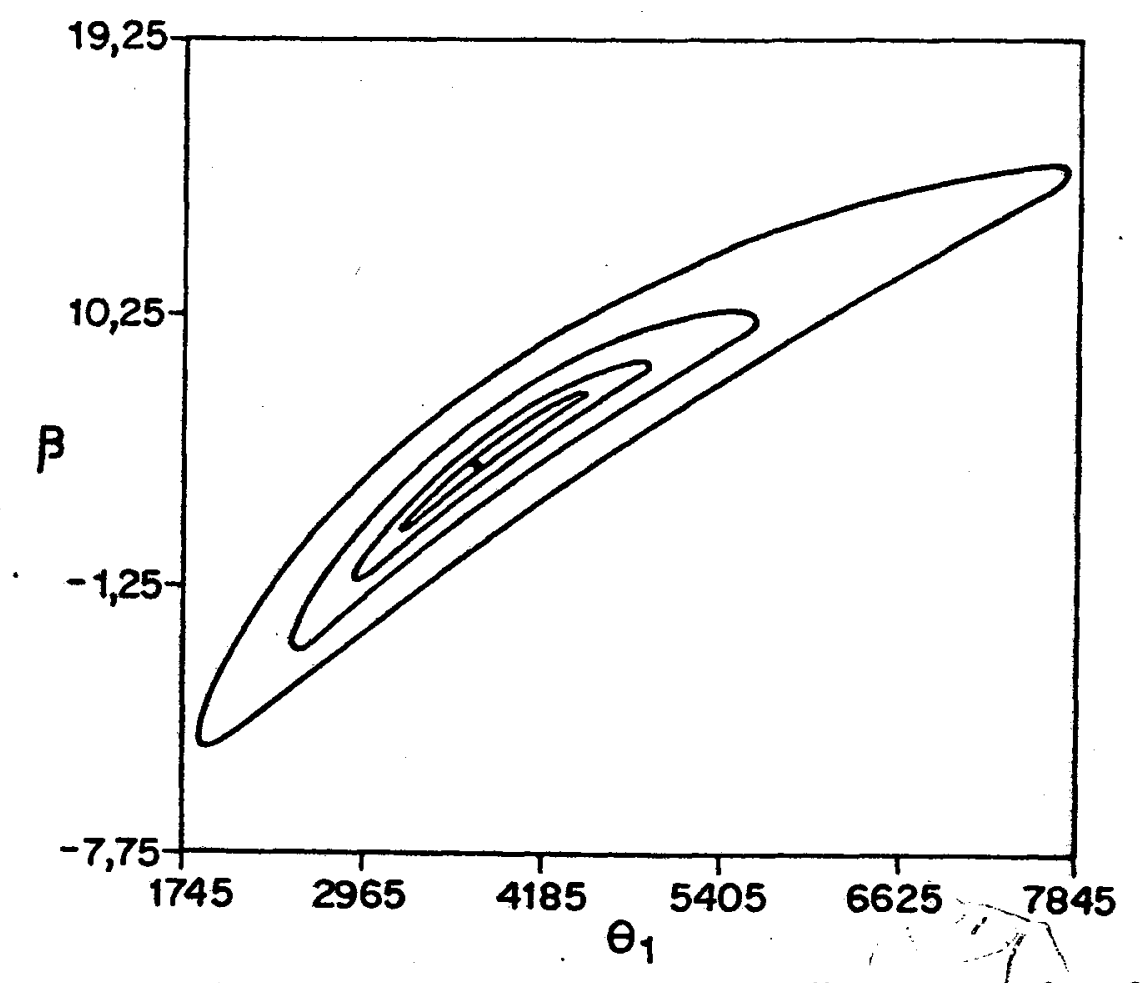

Figura 9.7: Contornos da função de verossimilhança para $\theta_{1}$ e $\beta$.

\subsubsection{O Efeito de Reparametrização na Análise Bayesiana do Modelo}

Em geral, o uso de reparametrização na análise Bayesiana de modelos estatísticos têm certas implicações práticas. Por exemplo, Hills (1987) estuda a eficiência da regra de Gauss-Hermite, usada em muitas rotinas de integração para determinar momentos a posteriori, e observa que essa regra pode não ser apropriada ou ineficiente quando a densidade a posteriori dos parâmetros de interesse não tem boa aproximação por uma densidade Normal multiplicada por um polinômio.

Quando da utilização de métodos de aproximação para integrais, o método 
de aproximação de Laplace (ver Apêndice 2) pode ter sua precisão comprometida se a densidade a posteriori de interesse não tem uma forma bem comportada (aproximadamente Normal) e a procura de uma parametrização adequada torna-se interessante.

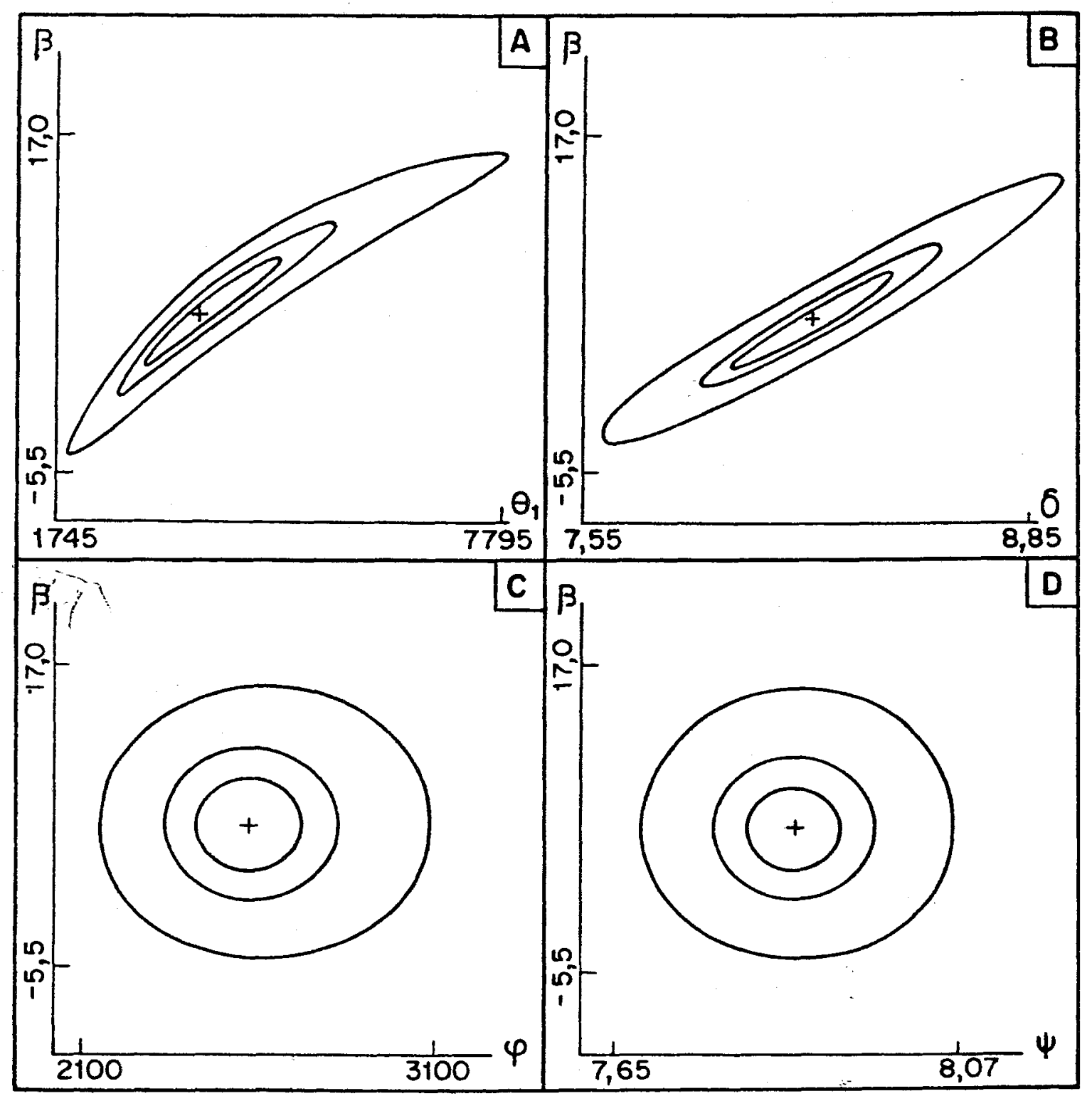

Figura 9.8: Contornos das densidades a posteriori conjuntas para $\left(\theta_{1}, \beta\right),(\delta, \beta),(\varphi, \beta) \mathrm{e}(\psi, \beta)$.

Neste contexto, na Figura 9.8, onde temos os contornos das densidades 
a posteriori conjuntas para os parâmetros $\left(\theta_{1}, \beta\right)$ (parametrização original), $(\delta=$ $\left.\log \theta_{1}, \beta\right)$ (reparametrização logarítmica em $\left.\theta_{1}\right),\left(\varphi=\theta_{1}^{-1} e^{\beta \overline{\bar{V}}}, \beta\right)$ (reparametrização ortogonal em $\left.\theta_{1}\right)$ e $\left(\psi=\log \left(\theta_{1}^{-1} e^{\beta \bar{V}}\right), \beta\right)$ (reparametrização ortogonal associada à logarítmica), podemos observar o efeito de reparametrização na análise Bayesiana do modelo, e apesar de não ser possível uma conclusão geral sobre transformações paramétricas em testes de sobrevivência acelerados considerando uma distribuição Exponencial, observe que na parametrização independente $(\psi, \beta)$, onde a matriz de informação de Fisher é diagonal e constante (ver (2.66)) e a densidade a priori é localmente uniforme (ver (3.15)), o método de aproximação de Laplace pode ter mais precisão do que quando trabalhamos na parametrização original $\left(\theta_{1}, \beta\right)$ cuja matriz de informação de Fisher não é diagonal, nem constante (ver (2.28))e a densidade a priori não é localmente uniforme (ver (3.10)), pois enquanto na Figura 9.8 (D) temos uma boa forma elíptica, na Figura 9.8 (A), essa forma não é observada.

\begin{tabular}{cc}
\hline$V_{j}$ & $L_{j}$ \\
\hline \hline 10 & 814,297 \\
20 & 317,088 \\
25 & 241,298 \\
30 & 194,490 \\
35 & 162,783 \\
40 & 132,914 \\
50 & 109,168 \\
\hline
\end{tabular}

Tabela 9.5: Valores requeridos $\overline{\mathrm{de}} L_{j}$ considerando-se $V_{j}$ fixo e $1-\gamma^{\prime}=0,80$.

\subsubsection{Controle de Qualidade de um Lote de Componentes}

Para achar o nível de estresse, $V_{j}$, e o período de tempo, $L_{j}$, requeridos em um teste de controle de qualidade, aplicamos os procedimentos obtidos no Capítulo 4, utilizando a densidade preditiva de uma observação futura dada em (4.9).

Com os dados da Tabela 9.1 temos $r=109 \mathrm{e}(\mathrm{de}(4.18)) c=1806870,2750$. 
Assumindn $\beta=5$ conhecido, $1-\gamma^{\prime}=0,80$ e um nível de estresse $V_{j}$ fixo, na Tabela 95 temos os valores de $L_{j}$, obtidos utilizando a expressão (4.20), considerando diferentes valores de $V_{j}$.

Considerando vários valores fixos de $L_{j}$, obtemos os valores dos níveis de estresse, $V_{j}$, necessários em um teste de controle de qualidade, através da expressão (4.21) $\operatorname{com} \beta=5$ e $1-\gamma^{\prime}=0,80$ (ver Tabela 9.6).

\begin{tabular}{ccc}
\cline { 2 - 2 } & \multicolumn{1}{c}{$V_{j}$} \\
\hline \hline 800 & 10,119 \\
700 & 11,080 \\
600 & 12,343 \\
500 & 14,087 \\
400 & 16,667 \\
350 & 18,492 \\
300 & 20,910 \\
250 & 24,274 \\
200 & 29,291 \\
150 & 37,608 \\
100 & 54,166 \\
Tabela 9.6: Valores de $V_{j}$ considerando-se $L_{j}$ fixo e $1-\gamma^{*}=0,80$.
\end{tabular}

Dessa forma, por exemplo, se o pesquisador industrial deseja verificar se os componentes de um determinado lote estão sob controle, considerando uma probabilidade de sobrevivência após $L_{j}$ igual a 0,80 , e tem para esse experimento $L_{j}=250$ unidades de tempo, pela Tabela 9.6, ele deve usar nesse teste um nível de estresse $V_{j}=24,274$ unidades de temperatura. Analogamente, se tecnicamente só é possível testar os componentes a 25 unidades de temperatura, pela Tabela 9.5, ele deve conduzir o experimento até 241,298 unidades de tempo. Em ambos os casos deve observar a proporção $p^{j}$ de componentes que falharam dentre as $m$ unidades em teste. Se $p^{j} \leq 0,20$, o lote de componentes está sob controle (ver seção 4.3). 


\subsection{Exemplo 2}

Considerando uma distribuição de Weibull para os tempos de sobrevivência e a relação estresse-resposta geral (6.1) que inclui o modelos usuais, Potência, Arrhenius e Eyring com uma variável estresse, aplicamos, aos dados deste exemplo, as metodologias Bayesianas de análise propostas nos Capitulos 6 e 7. Em particular consideramos a análise Bayesiana do modelo de Eyring com uma variável estresse desenvolvida no Capítulo 5.

$\mathrm{Na}$ Tabela 9.7 temos um conjunto de dados gerados considerando um modelo de Eyring com uma variável estresse e uma distribuição de Weibull com $\alpha=-12,7 ; \beta=6,2$ e $p=2$ (isto é, $\beta_{0}=12,7 ; \beta_{1}=6,2 ; X_{i}=\frac{1}{V_{i}}$ e $Z_{i}=-\log V_{i}$ no modelo geral $(6.1))$.

\begin{tabular}{rcccl}
\hline$i$ & $V_{i}$ & $n_{i}$ & $r_{i}$ & Observaçóes não-censuradas \\
\hline \hline 1 & 20 & 15 & 5 & $39,45,67,88,100$ \\
12 & 30 & 15 & 7 & $27,34,42,61,67,79,97$ \\
3 & 40 & 15 & 8 & $22,32,37,48,53,70,79,88$ \\
4 & 50 & 15 & 10 & $18,25,32,47,56,61,70,79,82,94$ \\
5 & 60 & 15 & 12 & $20,22,30,34,50,58,64,73,79,88,88,100$ \\
\hline
\end{tabular}

Tabela 9.7: Dados gerados com $\alpha-12,7, \beta=6,2$ e $p=2$.

Na Figura 9.9 temos o gráfico da densidade a posteriori marginal aproximada para $p$ (ver (5.15)), cuja moda a posteriori é dada por, $\tilde{p}=1,9864$. Um 
intervalo HPD aproximads $95 \%$ para $p$ é dado por $(1,4750 ; 2,5750)$.



Figura 9.9: Densidade a posteriori marginal para $p$.

Considerando $p$ conhecido $(p=1,9864)$ e como $T=T^{*^{p}}$ tem uma distribuição Exponencial com parâmetro $\lambda=\lambda^{* p}$, podemos considerar a análise Bayesiana padrão do modelo de Eyring com uma variável estresse (1.5) dada no Capítulo 3 (observar a transformação de escala envolvida - ver sub-seção 5.2.1).

Neste contexto, construimos (ver Figura 9.10) o gráfico da densidade a posteriori marginal para $\delta=\log \left(\theta_{1}\right)$ (dada em (3.14) considerando-se as devidas modificações de escala), onde $\theta_{1}$ é o tempo médio de sobrevivência sob o nível usual de estresse considerando a transformação de dados $T^{*^{1,9864}}$ com uma distribuição Exponencial com parâmetro $\lambda_{2}=\frac{1}{\theta_{1}}$. A moda dessa densidade a posteriori é dada por, $\tilde{\delta}=10,05612$ (isto é, $\tilde{\theta}_{1}=23297,9348$ ou $\tilde{\lambda}_{1}=0,00004292$ ). 
Voltando para a escala original dos dados, onde temos uma distribuição de sobrevivência de Weibull com parâmetro de escala $\lambda_{1}^{*}=\lambda_{1}^{\frac{1}{p}}, \tilde{\lambda}_{1}^{*}=0,006329$, o tempo médio de sobrevivência sob o nível usual de estresse $\theta_{1}^{*}=\Gamma\left(1+\frac{1}{p}\right) / \lambda_{1}^{*}$ é estimado por $\tilde{\theta}_{1}^{*}=140,0410$.



Figura 9.10: Densidade a posteriori marginal para $\delta=\log \left(\theta_{1}\right)$ com os dados transformados $T^{*^{1,9664}}$.

Considerando $p$ desconhecido, podemos obter inferências conjuntas para $\theta_{1}^{*}$ e $p$ através da densidade a posteriori conjunta para $\theta_{1}^{*}$ e $p$ dada em (5.19). Na Figura 9.11 temos o gráfico dos contornos HPD aproximados $95 \%, 90 \%, 70 \%$ e $50 \%$ dessa densidade a posteriori, cuja a moda a posteriori é dada por, $\tilde{\theta}_{1}^{*}=128,1200 \mathrm{e}$ $p=2,0455$.

Considerando a escala logarítimica para $\theta_{1}^{*}$ e $p\left(\delta^{*}=\log \left(\theta_{1}^{*}\right)\right.$ e $\left.\gamma^{*}=\log (p)\right)$ construímos, na Figura 9.12, o gráfico dos contornos HPD aproximados 95\%, 90\%, 
700\% e $50 \%$ da densidade a posteriori conjunta para $\delta^{*}$ e $\gamma^{*}$, onde podemos observar uma boa forma elíptica. A moda dessa densidade a posteriori é dada por, $\tilde{\delta}^{*}=4,88503\left(\tilde{\theta}_{1}^{*}=131,7005\right)$ e $\gamma^{*}=0,72506(\tilde{p}=2,06485)$. Um intervalo HPD aproximado $95 \%$ para $p$ é dado por $(1,5863 ; 2,6209)$.

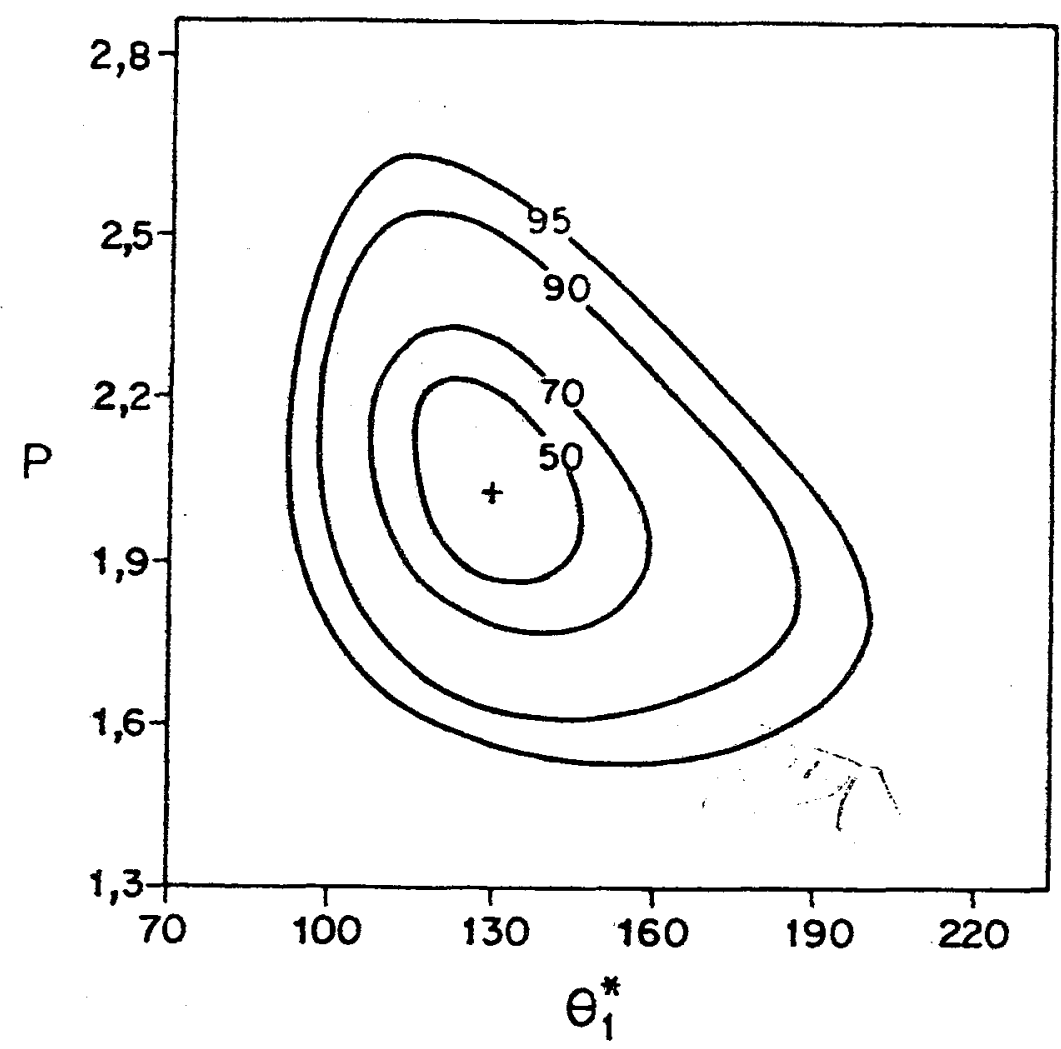

Figura 9.11: Contornos HPD 95\%, 90\%, 70\% e 50\% da densidade a posteriori conjunta para $\theta_{1}^{*}$ e $p$.

$\mathrm{Na}$ Tabela 9.8 temos os intervalos HPD aproximados $95 \%$ para $\theta_{1}$ considerando $p$ conhecido ou desconhecido. Observamos que os resultados são relativamente próximos.

\begin{tabular}{cl}
\hline $\mathrm{p}$ & Intervalos HPD 95\% para $\theta_{1}^{*}$ \\
\hline \hline conhecido & $(99,1935 ; 214,6563)$
\end{tabular}

desconhecido $(92,1299 ; 205,2031)$

Tabela 9.8: Intervalos HPD aproximados $95 \%$ para $\theta_{1}^{*}$. 


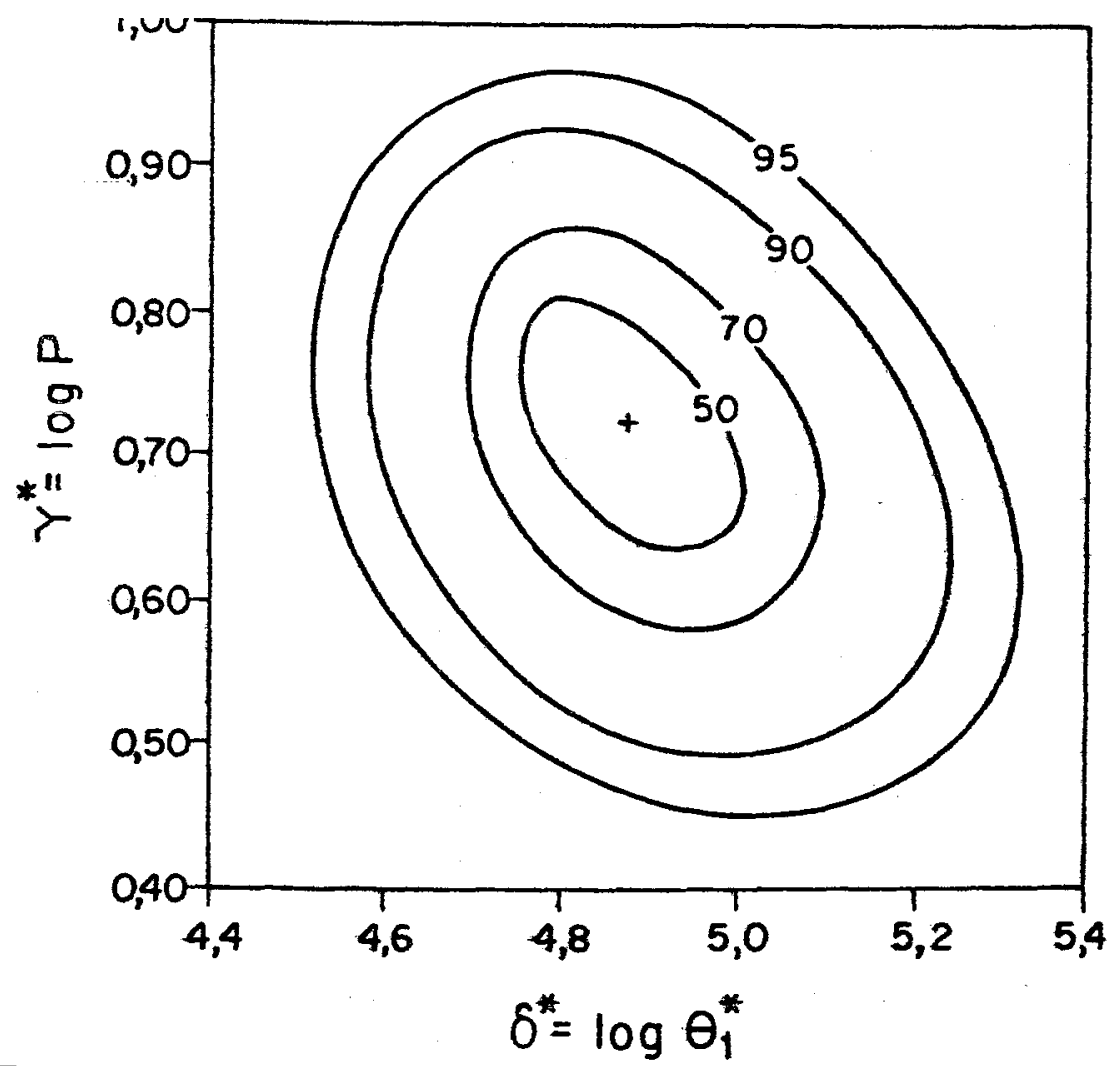

Figura 9.12: Contornos HPD 95\%, 90\%, $70 \%$ e $50 \%$ da densidade a posteriori conjunta para $\delta^{*}=\log \left(\theta_{1}^{*}\right)$ e $\gamma^{*}=\log (p)$.

Observe que no desenvolvimento desse exemplo, apesar de termos considerado as expressões do Capítulo 5 , e por vezes do 3 , os mesmos resultados poderiam ser obtidos considerando-se as expressões do Capítulo 6.

Na Figura 9.13 temos os gráficos das densidades a posteriori marginais para a função de confiabilidade $R_{1}^{*}$, assumindo $p=1,9864$ conhecido, dada em (6.20), para $t_{1}^{*}$ igual a 50,95 e 130 , cujas modas a posteriori são dadas por $\tilde{R}_{1}^{*}(50)=$ $0,9154, \tilde{R}_{1}^{*}(95)=0,7195$ e $\tilde{R}_{1}^{*}(130)=0,5247$, respectivamente (observe que para a construção desses gráficos consideramos os dados transformados $T^{*^{1,9864}}$; também, as alturas das curvas são iguais porque consideramos as "densidades a posteriori 
relativas" (ver Apêndice 4)).

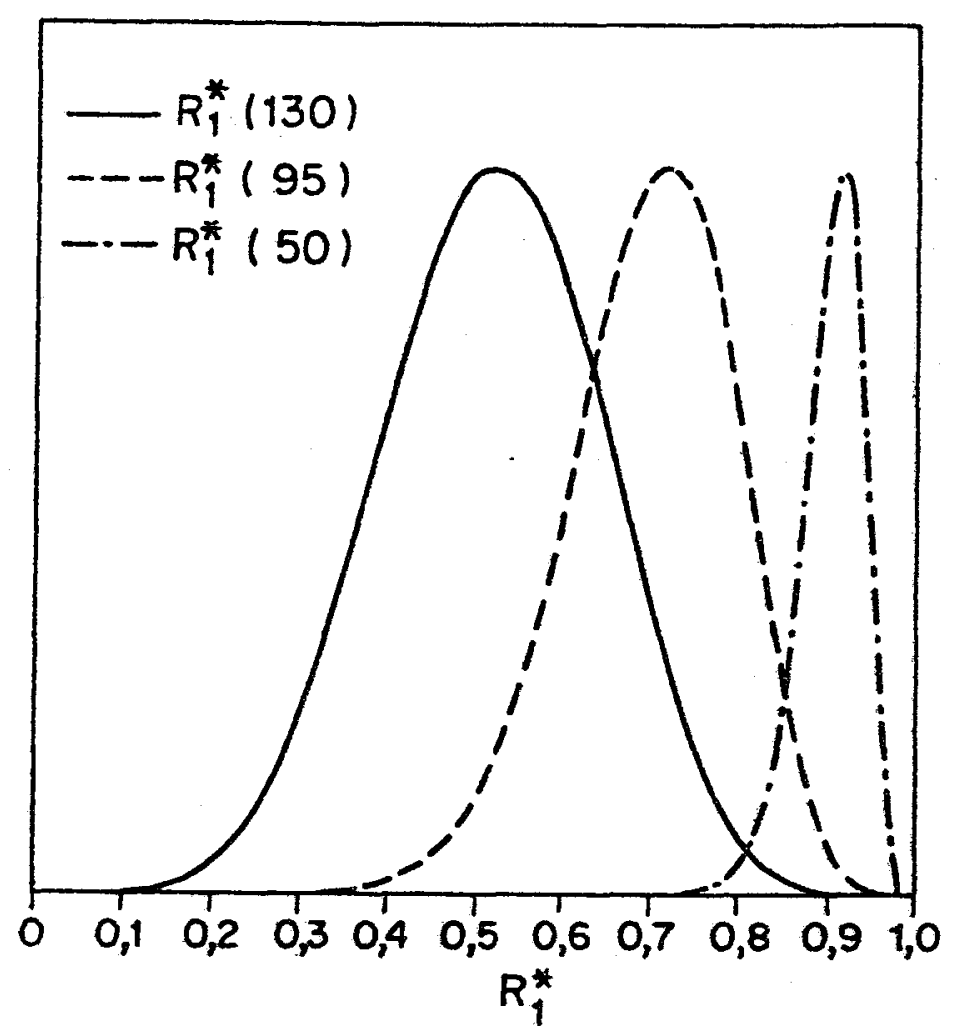

Figura 9.13: Densidades a posteriori para $R_{1}^{*}$.

Considerando os niveis'de estresse $V_{2}=30$ e $V_{4}=50$ (ver Tabela 9.7), aplicamos a seguir os procedimentos Bayesianos de análise da relação estresse-resposta (6.1), desenvolvidos no Capítulo 7, direcionados às situações onde não é possível a condução de experimentos considerando-se o nivel usual de estresse. Os resultados são comparados com os resultados obtidos considerando-se o experimento acelerado com $V_{1}=20, V_{2}=30$ e $V_{4}=50($ ver tabela 9.7$)$.

Na Figura 9.14 temos os gráficos das densidades a posteriori marginais para o parâmetro $p$ tanto considerando o nível usual de estresse no experimento (isto é, experimento com $V_{1}=20, V_{2}=30$ e $V_{4}=50$ ), como não o considerando (isto é, experimento somente com $V_{2}=30$ e $\left.V_{4}=50\right)$ (ver (6.7) e (7.6), respectivamente). Denotando por $\tilde{p}_{c}$ e $\tilde{p}_{s}$ as modas dessas densidades a posteriori, respectivamente, 
temos que $\tilde{p}_{c}=1,9627$ e $\tilde{p}_{s}=1,8272$.

Um Intervalo HPD aproximado $95 \%$ para $p$ quando consideramos $V_{1}=20$ no experimento é dado por, $(1,2847 ; 2,8361)$ e um intervalo HPD aproximado $95 \%$ para $p$ quando $V_{1}=20$ não é considerado é dado por, $(1,1875 ; 2,8194)$.

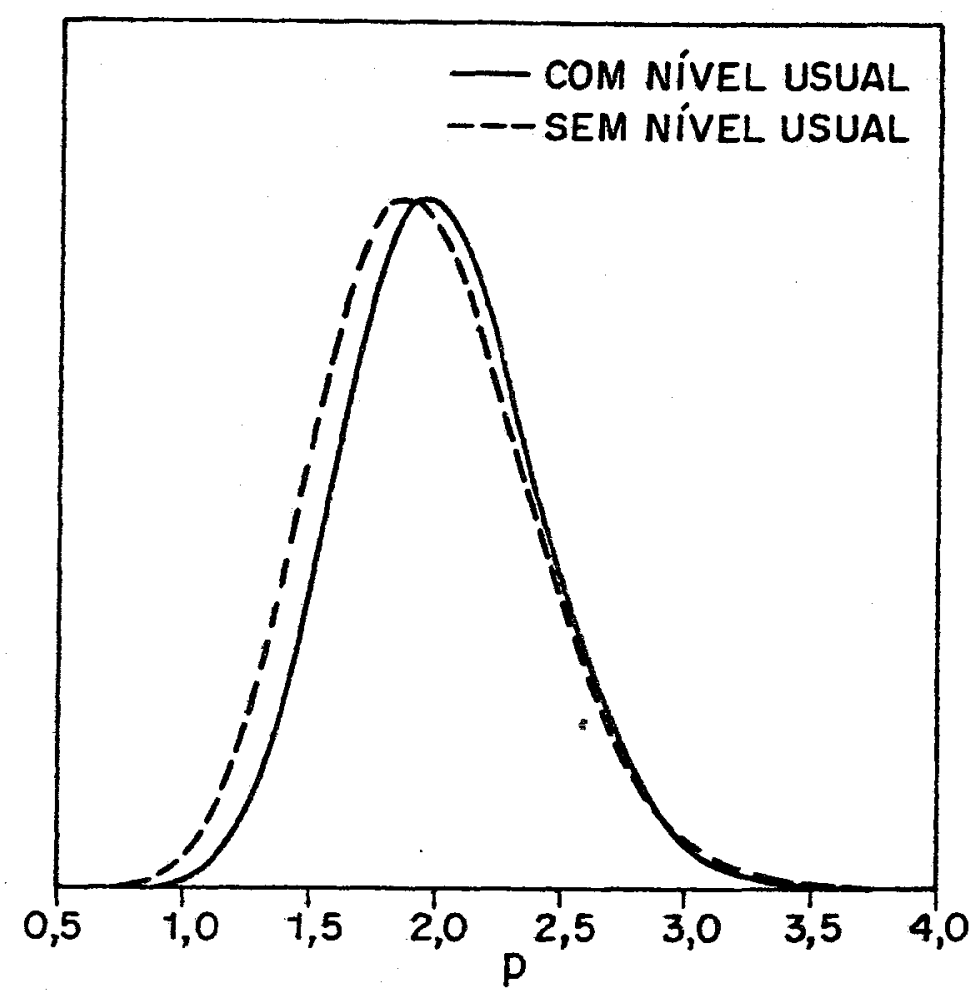

Figura 9.14: Densidades a posteriori marginais para $p$.

Como $T^{*^{p}}$ tem uma distribuição Exponencial com parâmetro $\lambda=\lambda^{*^{p}}$, de forma análoga a que fizemos anteriormente quando tínhamos cinco níveis de estresse no experimento, desenvolvemos uma análise Bayesiana assumindo $p$ conhecido, isto é, consideramos as transformações de dados, $T^{*^{p c}}=T^{*^{1,9627}}$ (quando $V_{1}=20$ é considerado) e $T^{*_{s}}=T^{*^{1,8782}}$ (quando consideramos somente $V_{2}=30$ e $V_{4}=50$ ). $\mathrm{Na}$ Figura 9.15 temos o gráfico das densidades a posteriori marginais para $\delta=\log \left(\theta_{1}\right)$ em ambos os casos (ver (6.14) e (7.19), respectivamente, na escala logarítimica e considerando as transformações de dados), onde $\theta_{1}$ é o tempo médio de sobrevivência 
sob o nivel de estresse usual, $V_{1}$, considerando-se os dados transformados $T^{*^{1,962 t}} \mathrm{e}$ $T^{*^{1, \mathbf{B} 782}}$ com distribuição Exponecial. As modas dessas densidades a posteriori são dadas, respectivamente, por, $\tilde{\delta}_{c}=9,9548\left(\tilde{\theta}_{1_{c}}^{*}=141,4009\right)$ e $\tilde{\delta}_{s}=9,4678\left(\tilde{\theta}_{1_{s}}^{*}=\right.$ $137,2467)\left(0_{1}^{*}=\Gamma\left(1+\frac{1}{p}\right) / \lambda_{1}^{*}\right.$, na escala original, onde os dados tem uma distribuição de Weibull com parâmetros de escala $\left.\lambda_{1}^{*}=\lambda_{1}^{\frac{1}{p}}\right)$.

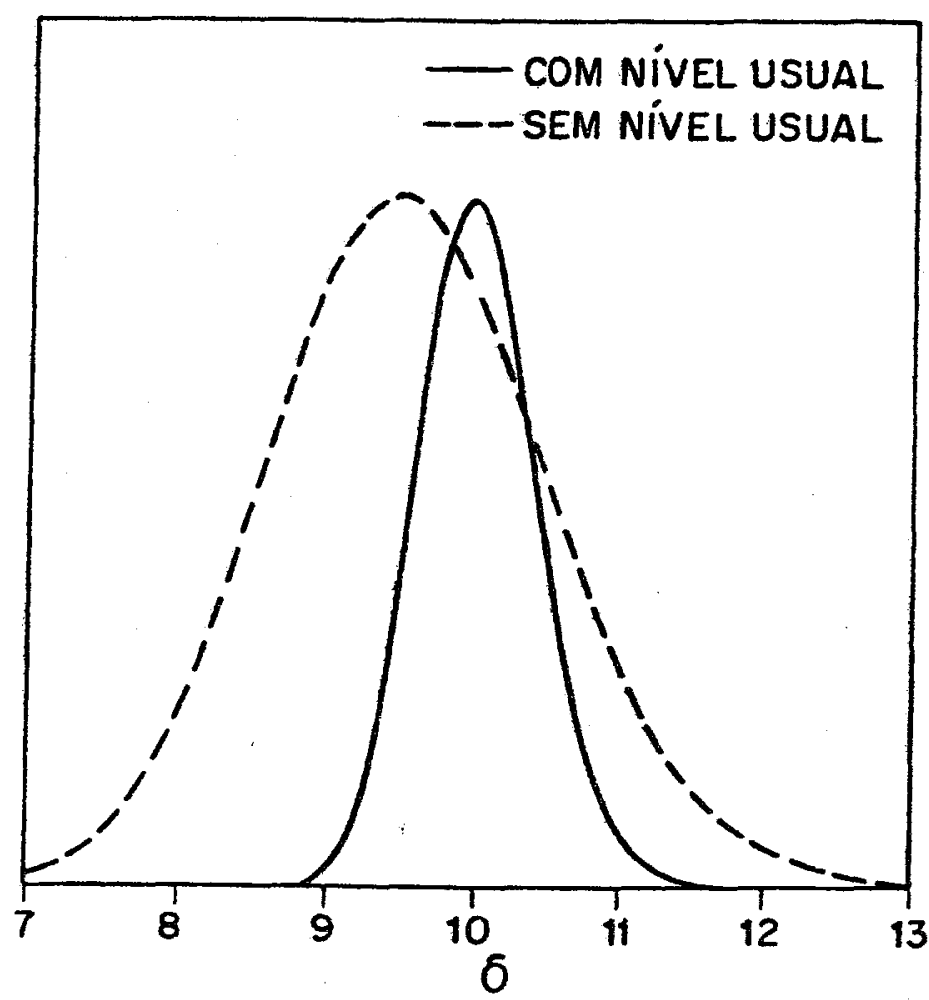

Figura 9.15: Densidades a posteriori marginais para $\delta=\log \left(\theta_{1}\right)$ (com os dados transformados $T^{*^{1,9627}}$ e $T^{*^{1,8782}}$ ).

\begin{tabular}{cl}
\hline$V_{1}=20$ & Intervalos HPD 95\% para $\theta_{1}^{*}$ \\
\hline é considerado & $(98,6004 ; 222,4494)$
\end{tabular}
não é considerado $(54,1836 ; 404,9531)$

Tabela 9.9: Intervalos HPD aproximados $95 \%$ para $\theta_{1}^{*}$ considerando ou não $V_{1}=20$ no experimento e assumindo $p$ conhecido.

Na Tabela 9.9 temos os intervalos HPD aproximados $95 \%$ para o tempo 
médin de sibrevivência, $\theta_{1}^{*}$, considerando ou não considerando o nivel $V_{1}=20$ no experimento.

Observe que quando não consideramos o nivel usual de estresse no experimento, o intervalo HPD para o tempo médio de sobrevivência, $\theta_{1}^{*}$, é bem maior do que o obtido quando esse nível é utilizado no teste (ver Tabela 9.9). Além disso, considerando os dados dos níveis $V_{1}=20, V_{2}=30$ e $V_{4}=50$, que são uma proporção dos dados utilizados no ínicio do exemplo quando os cinco niveis da Tabela 9.7 foram considerados, obtemos, considerando $p$ conhecido, um intervalo HPD $95 \%$ para $\theta_{1}^{*}$ próximo do intervalo HPD obtido considerando-se $V_{1}, V_{2}, V_{3}, V_{4}$ e $V_{5}$ no experimento (ver Tabelas 9.8 e 9.9).

\subsection{Exemplo 3}

Considere que certos componentes eletrônicos têm uma distribuição de sobrevivência Exponencial sob todos os ambientes possiveis. Com o objetivo de inferir sobre o tempo médio de sobrevivência dos componentes nas condições usuais de funcionamento (temperatura de $30^{\circ} \mathrm{Ce} 100 \mathrm{v}$ de voltagem), formula-se um experimento acelerado, considerando-se voltagem combinada com temperatura como mecanismo básico de aceleração de falha. Em cada combinação voltagem-temperatura coloca-se 10 componentes em teste e o teste termina quando 6 falhas são observadas. Na tabela 9.10 temos 12 conbinações de estresse $\left(V_{i}, U_{i}\right), i=1,2, \ldots, 12$, onde $V_{i}$ é o i-ésimo nível de temperatura e $U_{i}$ é o i-ésimo nível de voltagem, $n_{i}=10, r_{i}=6$; também temos os estimadores de máxima verossimilhança para $\theta_{i}=\frac{1}{\lambda_{i}}$. 


\begin{tabular}{rrrrl}
$i$ & $V_{i}$ & $U_{i}$ & $0_{i}$ & Observaçôes \\
\hline 1 & 30 & 100 & 1500 & $400,600,650,700,800,1170,1170+, 1170+, 1170+, 1170+$ \\
2 & 30 & 200 & 1400 & $300,450,500,600,620,1186,1186+, 1186+, 1186+, 1186+$ \\
3 & 30 & 300 & 1100 & $250,300,400,500,800,1320,1320+, 1320+, 1320+, 1320+$ \\
4 & 30 & 400 & 1000 & $200,300,350,500,650,800,800+, 800+, 800+, 800+$ \\
5 & 40 & 100 & 1400 & $400,500,600,650,800,1090,1090+, 1090+, 1090+, 1090+$ \\
6 & 40 & 200 & 1100 & $200,300,350,500,700,910,910+, 910+, 910+, 910+$ \\
7 & 40 & 300 & 1000 & $250,300,350,500,700,780,780+, 780+, 780+, 780+$ \\
8 & 40 & 400 & 900 & $150,250,380,400,500,744,744+, 744+, 744+, 744+$ \\
9 & 50 & 100 & 1100 & $180,260,350,480,750,916,916+, 916+, 916+, 916+$ \\
10 & 50 & 200 & 1000 & $200,280,320,460,780,792,792+, 792+, 792+, 792+$ \\
11 & 50 & 300 & 900 & $150,220,310,470,680,714,714+, 714+, 714+, 714+$ \\
12 & 50 & 400 & 800 & $120,180,280,360,620,648,648+, 648+, 648+, 648+$ \\
\hline
\end{tabular}

Tabela 9.10: Tempos de sobrevivências dos componentes em horas (dados com ' + ' são censurados).

Assumindo que o modelo de Eyring generalizado (1.6) é apropriado para o conjunto de dados da Tabela 9.10, podemos estimar os parâmetros do modelo, $\alpha, \beta, \gamma$ e $\delta$, usando o método de mínimos-quadrados. De (8.3) temos,

$$
\log \left(\frac{\hat{\lambda}_{i}}{V_{i}}\right)=\alpha_{1}+\alpha_{2} U_{i}+\alpha_{3} \frac{U_{i}}{k_{1} V_{i}}+\alpha_{4} \frac{1}{k_{1} V_{i}}+\xi_{i}
$$

onde $\hat{\lambda}_{i}$ são os estimadores de máxima verossimilhança de $\lambda_{i}=\frac{1}{\theta_{i}}, i=1,2, \ldots, k, \alpha_{1}=$ $\log \alpha, \alpha_{2}=\gamma, \alpha_{3}=\delta$ e $\alpha_{4}=-\beta$.

Os estimadores de minimos-quadrados (EMQ) $\hat{\alpha}_{1}, \hat{\alpha}_{1}, \hat{\alpha}_{2}, \hat{\alpha}_{3}$ e $\hat{\alpha}_{4}$ (ver Tabela 9.11) têm uma distribuição Normal assintótica dada por, $N\left\{\underline{\alpha} ; \sigma^{2}\left(X^{\prime} X\right)^{-1}\right\}$, onde,

$$
\left(X^{\prime} X\right)^{-1}=\left[\begin{array}{cccc}
11,7720 & -0,0390 & 1,9860 \times 10^{-16} & -5,9580 \times 10^{-14} \\
\vdots & 0,0002 & -7,9430 \times 10^{-19} & 1,9860 \times 10^{-16} \\
\vdots & & 4,1980 \times 10^{-33} & -1,0500 \times 10^{-30} \\
\text { simétrica } & & & 3,1490 \times 10^{-28}
\end{array}\right]
$$


O estimador de $\sigma^{2}$ baseado na soma de quadrados dos resíduos (SQR) é dadn por, $\sigma^{2}=S Q R /(k-4)=0,01280 / 8=0,0016$ (ver por exemplo, Draper e Smith, 1981).

\begin{tabular}{ccc}
\hline EMQ para & EMQ para & Intervalo de confiança 95\% \\
$\alpha_{1}, \alpha_{2}, \alpha_{3}, \alpha_{4}$ & $\alpha, \beta, \gamma, \delta$ & para $\alpha, \beta, \gamma, \delta$ \\
\hline \hline$\hat{\alpha}_{1}=-11,282$ & $\hat{\alpha}=e^{\alpha_{1}}=1,259 \times 10^{-5}$ & $(0,0000092 ; 0,0000173)$ \\
$\hat{\alpha}_{2}=-0,000603$ & $\hat{\gamma}=\hat{\alpha}_{3}$ & $(-0,0005546 ; 0,0017614)$ \\
$\hat{\alpha}_{3}=3,75 \times 10^{-18}$ & $\hat{\delta}=\hat{\alpha}_{3}$ & $\left(-2,2385 \times 10^{-18} ; 9,7389 \times 10^{-18}\right)$ \\
$\hat{\alpha}_{4}=-1,593 \times 10^{-15}$ & $\hat{\beta}=\hat{\alpha}_{4}$ & $\left(-3,2333 \times 10^{-15} ; 0,0469 \times 10^{-15}\right)$ \\
\hline
\end{tabular}

Tabela 9.11: Estimadores de mínimos-quadrados e intervalos de confiança $95 \%$ para $\alpha, \beta, \gamma$ e $\delta$.

$\mathrm{Na}$ Tabela 9.11 temos os estimadores de mínimos-quadrados e os intervalos de confiança $95 \%$ para $\alpha, \beta, \gamma$ e $\delta$.

Com os estimadores de mínimos-quadrados para $\alpha_{1}, \alpha_{2}, \alpha_{3}$ e $\alpha_{4}$ achamos um estimador de $\lambda_{1}=V_{1} \exp \left\{\alpha_{1}+\alpha_{2} U_{1}+\alpha_{3} \frac{U_{1}}{k_{1} V_{1}}+\alpha_{4} \frac{1}{k_{1} V_{1}}\right\}$, onde $V_{1}=30$ e $U_{1}=100$, dado (de (8.5)) por, $\hat{\lambda}_{1}=0,0006450$. Dessa forma, um estimador para o tempo médio de sobrevivência dos componentes sob os níveis usuais é dado por $\hat{\theta}_{1}=\frac{1}{\hat{\lambda}_{1}}=$ 1549,1900 .

Um intervalo de confiança $95 \%$ para $\lambda_{1}$ é dado (de (8.6)) por, $(0,0005740$; 0,0007260 ), isto é, um intervalo de confiança aproximado $95 \%$ para $\theta_{1}=\frac{1}{\lambda_{1}}$ é dado por, $(1376,710 ; 1743,2680)$.

Observe que este intervalo de confiança para $\theta_{1}$ é determinado a partir do modelo (8.2) que considera $\log \left(\hat{\lambda}_{i}\right)$ como a variável resposta, onde $\hat{\lambda}_{i}$ é o estimador de máxima verossimilhança para $\lambda_{i}$ em cada combinação de estresse, $\mathbf{e}$ um modelo de erro multiplicativo, $\hat{\lambda}_{i}=\lambda_{i} \cdot \xi_{i}$. Dessa forma, uma análise Bayesiana do modelo de Eyring generalizado pode ser de grande interesse prático. 
-Na Figura 9.16 temos o gráfico de um contorno da densidade a posteriori conjunta (8.13) para $\gamma$ e $\delta$ aproximada pelo método de Laplace completamente exponencial. A moda dessa densidade a posteriori é dada por $\tilde{\gamma} \cong 0,0005590 \mathrm{e}$ $\tilde{\delta} \cong 5,2020 \times 10^{-18}$.

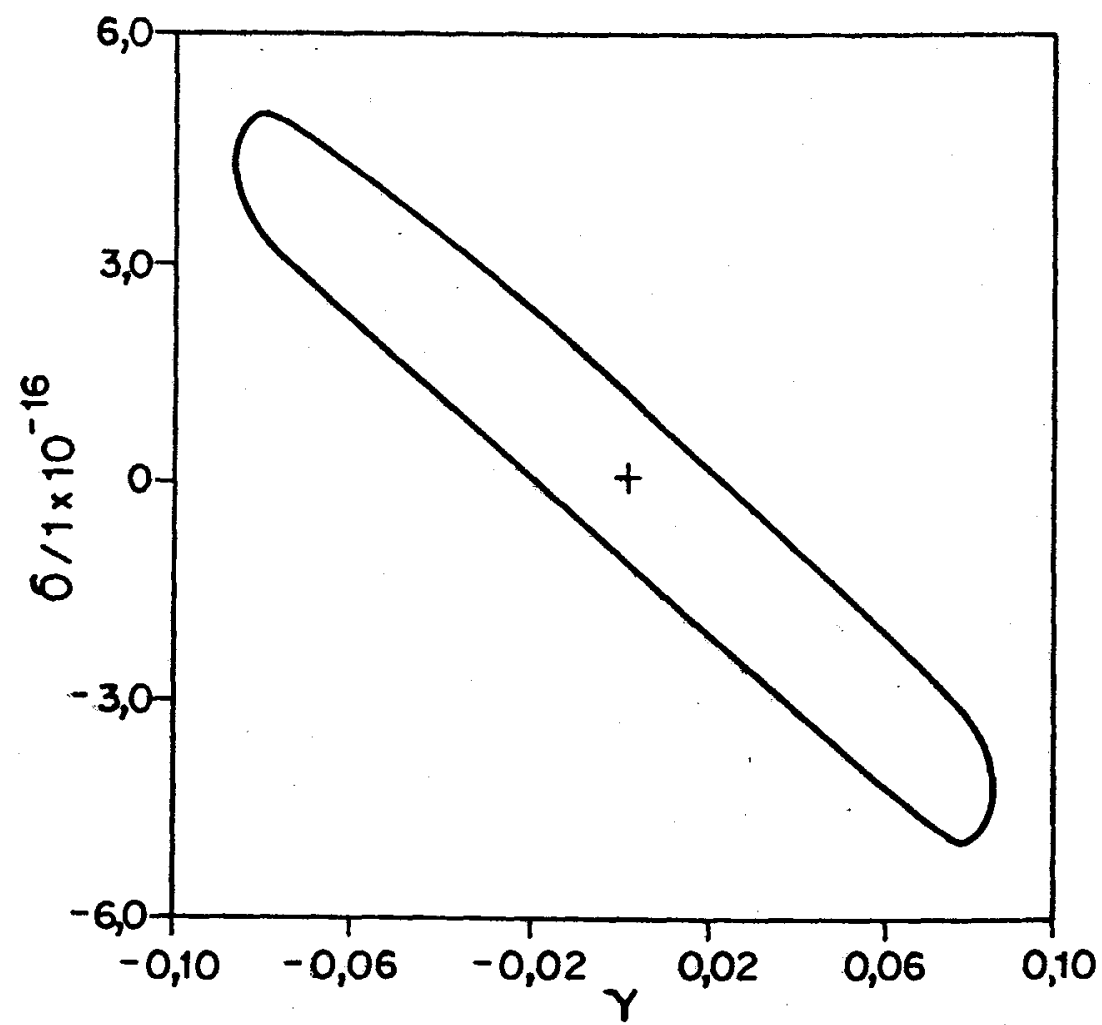

Figura 9.16: Contornos da densidade a posteriori conjunta para $\gamma$ e $\delta$.

Assumindo $\gamma$ e $\delta$ conhecidos $\left(\gamma=0,0005590\right.$ e $\left.\delta=5,2020 \times 10^{-18}\right)$ temos na Figura 9.17 o gráfico da densidade a posteriori marginal para $\beta$ dada em (8.16). A moda dessa densidade a posteriori é dada por $\tilde{\beta}=-1,2178 \times 10^{-15}$. 


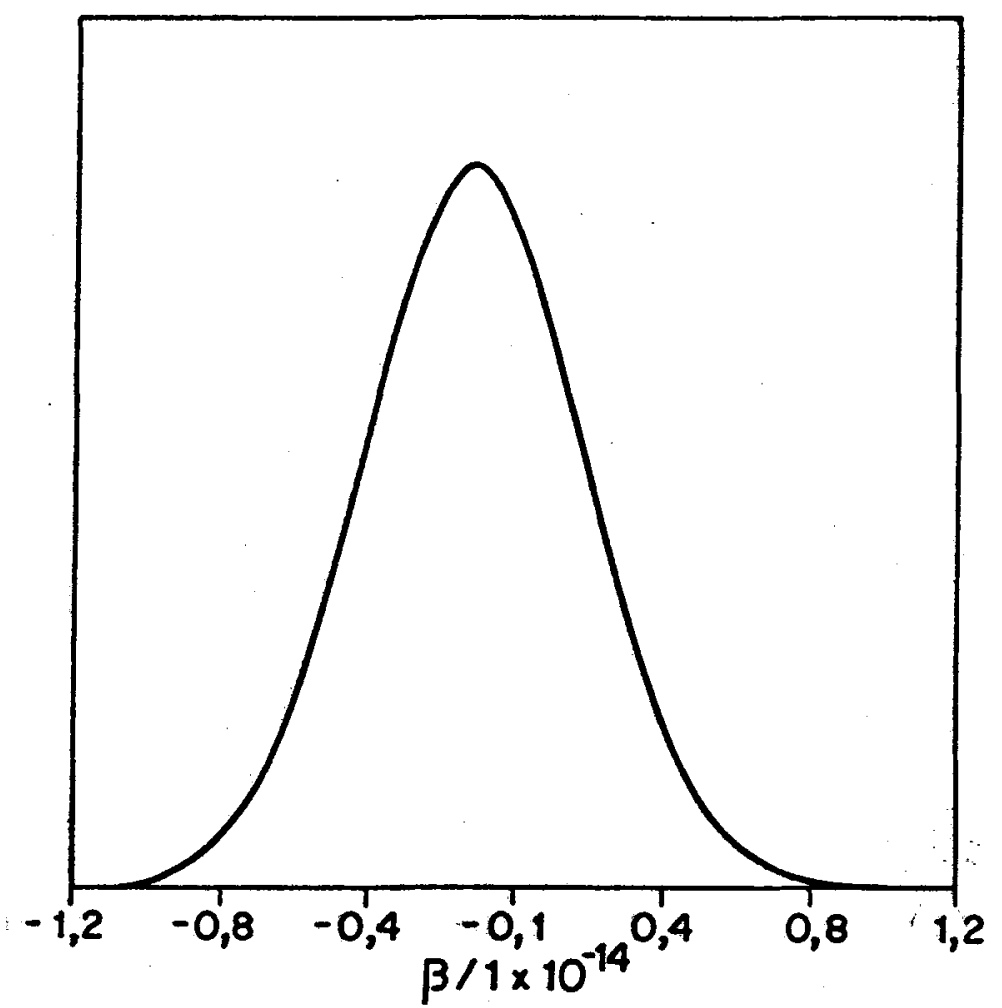

Figura 9.17: Densidade a posteriori marginal para $\beta$ assumindo $\gamma$ e $\delta$ conhecidos.

Na Figura 9.18 temos o gráfico da densidade a posteriori para $\theta_{1}$ dada em (8.20) assumindo $\gamma=0,0005590$ e $\delta=5,2020 \times 10^{-18}$ conhecidos e aproximada pelo método de Laplace completamente exponencial. A moda dessa densidade a posteriori é dada por $\tilde{\theta}_{1}=1553,33$. Um intervalo HPD $95 \%$ aproximado para $\theta_{1}$ é dado por, $(1060,00 ; 2317,00)$ (ver Figura 9.18) o qual é diferente do intervalo de confiança $95 \%$ dado por, $(1376,7130 ; 1743,2680)$, obtido considerando-se (8.6).

Assumindo $\gamma=0,0005590, \delta=5,2020 \times 10^{-18}$ e $\beta=-1,2178 \times 10^{-15}$ conhecidos, construímos na Figura 9.19, o gráfico da densidade a posteriori para $\alpha$ dada em (8.25). A moda dessa densidade a posteriori é dada por $\tilde{\alpha}=1,2575 \times 10^{-5}$. 
Finalmente, na Figura 9.20, temos o gráfico da densidade a posteriori para $\theta_{1}$ assumindo $\gamma, \delta$ e $\beta$ conhecidos dada em (8.26). A moda dessa densidade a posteriori é dada (de (8.27)) por $\tilde{\theta}_{1}^{\prime}=1602,0543$ e um intervalo HPD aproximado $95 \%$ para $\theta_{1}$ é dado (ver (8.28)) por, $(1276,2688 ; 2021,8559)$.

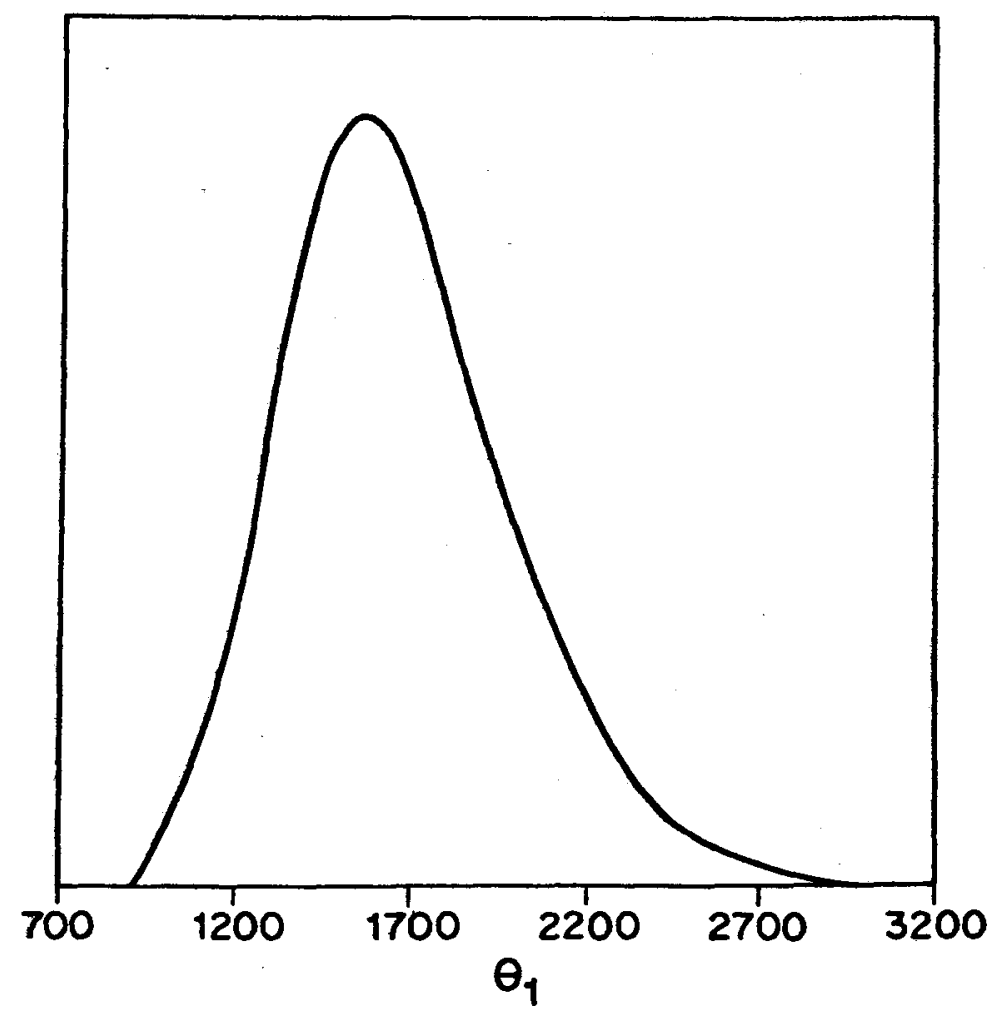

Figura 9.18: Densidade a posteriori para $\theta_{1}$ assumindo $\gamma$ e $\delta$ conhecidos. 


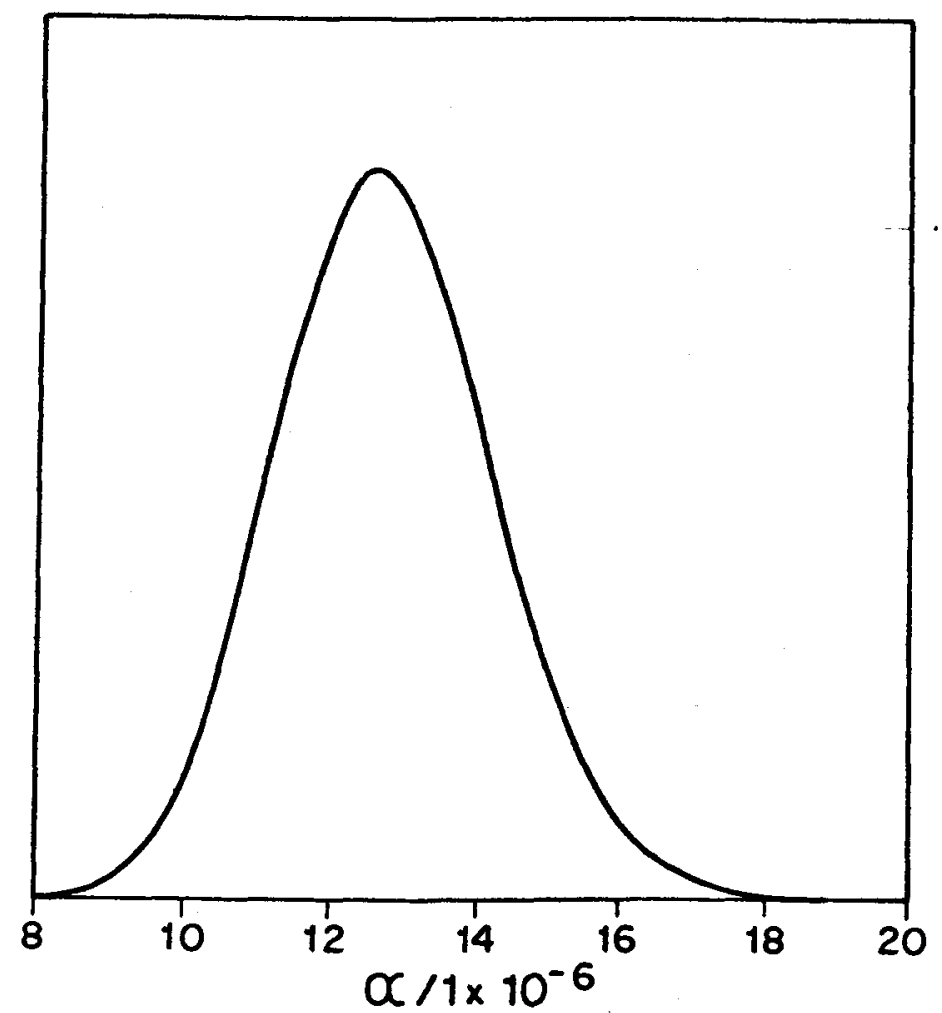

Figura 9.19: Densidade a posteriori para $\alpha$ assumindo $\gamma, \delta$ e $\beta$ conhecidos.

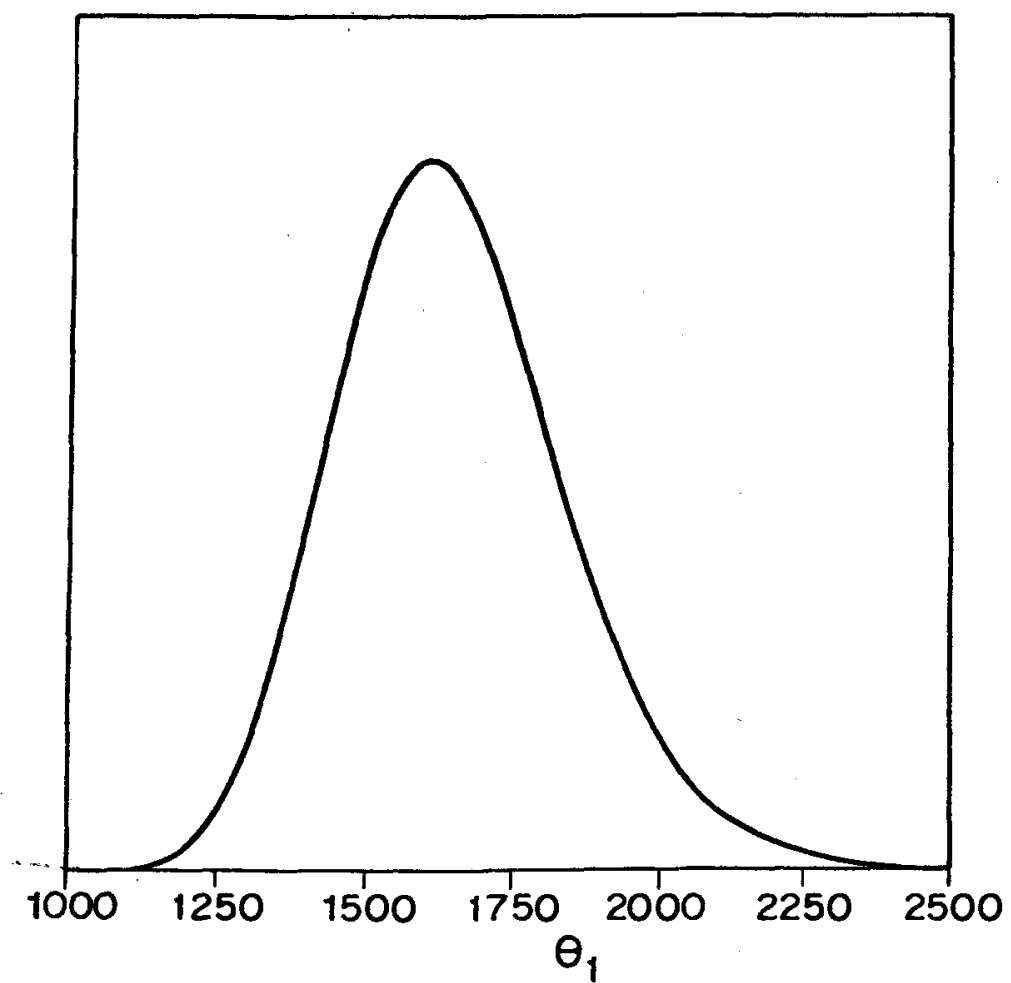

Figura 9.20: Densidade a posteriori para $\theta_{1}$ assumindo $\gamma, \delta$ e $\beta$ conhecidos. 


\section{Capítulo 10}

\section{Conclusões Finais e Perspectivas Futuras}

Em todo o desenvolvimento desta dissertação, assumindo uma distribuição de Weibull para os tempos de sobrevivência, e em particular, uma distribuição Exponencial, podemos observar que o uso de métodos Bayesianos em testes de sobrevivência acelerados podem ser de grande interesse prático tanto na análise do modelo de Eyring com uma variável estresse, quanto na análise de relação estresse-resposta geral (6.1) que tem como casos particulares, além do modelo de Eyring com uma variável estresse, os modelos de Potência e Arrenhius.

Esses procedimentos Bayesianos permitem a obtenção de inferências sobre - tempo médio de sobrevivência, $\theta_{1}$, sob o nível usual de estresse, $V_{1}$, com boa precisão e tornam-se uma boa alternativa aos métodos clássicos assintóticos usuais. Também, inferências sobre a função de confiabilidade dos componentes sob o nível usual estresse podem ser obtidas. Além disso, usando a densidade preditiva de uma observação Futura, pudemos desenvolver um procedimento para controle de qualidade de um lote de componentes que têm grande aplicabilidade em indústrias de várias áreas e através do qual o pesquisador tem liberdade para decidir por qual estratégia deve adotar em cada caso particular. 
Como, em muitas situações práticas, não é possível a condução de um teste acelerado que considera o nível usual da variável física que estressa o componente, pudemos desenvolver (ver Capítulo 7) uma Análise Bayesiana da relação estresseresposta geral (6.1) direcionada a essas situações, e inferências sobre o tempo médio de sobrevivência sob o nível usual de estresse podem ser obtidas considerando um experimento acelerado somente com niveis de estresse maiores que o usual.

Do ponto de vista da precisão do método de Laplace completamente exponencial nas aproximações das densidades a posteriori de interesse, pudemos observar que, em geral, as reparametrizações que "levam" a uma densidade a priori localmente uniforme melhoram sensivelmente a precisão das aproximações.

Observamos que a reparametrização ortogonal tem efeito relevante na Análise Bayesiana dos modelos usuais de teste de sobrevivência acelerado, pois, como proporciona a obtenção de parâmetros não-correlacionados, utilizando-a, conseguimos obter um estimador Bayesiano, sob "forma fechada", para o tempo médio de sobrevivência, $\theta_{1}$, sob nível usual de estresse e inferências por intervalo sobre esse parâmetro com grande simplicidade.

Finalmente, apesar do modelo de Eyring generalizado ter uma forma mais elaborada por considerar a possibilidade de termos componentes sob níveis de duas váriaveis estresse, conseguimos desenvolver uma Análise Bayesiana deste modelo, $\mathrm{e}$ pelos resultados obtidos pudemos observar que essa análise é mais atrativa do que a análise clássica via método de mínimos-quadrados. 


\subsection{Perspectivas de Trabalhos Futuros}

O uso de uma Parametrização Independente na Determinação de um Procedimento de Controle de Qualidade

Como visto no Capítulo 4, a determinação de um procedimento de controle de qualidade quando o parâmetro $\beta$ é desconhecido envolve o uso de metodologia numérica, introduzindo, de certa forma, dificuldade adicional ao problema.

Alternativamente, futuramente, poderemos nos empenhar no desenvolvimento e estudo de um procedimento de controle de qualidade, obtido via reparametrização ortogonal (ver por exemplo, Cox e Reid, 1987) adotada para o parâmetro $\theta$, ou então, através da associação dessa reparametrização à reparametrização logarítmica adotada para o parâmetro ortogonal obtido.

Por hora, apresentamos a seguir alguns resultados preliminares, obtidos via reparametrização ortogonal em $\theta$ associada a logarítmica.

Considerando as expressōes (2.2) e (2.6), sob um determinado nivel de estresse $V_{j}$ fixo, $\theta_{j}=e^{\beta / V_{j}} / V_{j} e^{\alpha}$, isto é ,

$$
\alpha=\log \left(\frac{e^{\beta / V_{j}}}{\theta_{j} V_{j}}\right) .
$$

Substituindo (10.1) em (2.7), a função de verossimilhança para $\theta_{j}$ e $\beta$ é dada por,

$$
L\left(\theta_{j}, \beta\right) \propto \theta_{j}^{-r} \exp \left\{-\beta\left(a_{1}-\frac{r}{V_{j}}\right)-\frac{1}{\theta_{j}} \sum_{i=1}^{k} \frac{V_{i}}{V_{j}} A_{i} e^{\beta\left(\frac{1}{V_{j}}-\frac{1}{V_{i}}\right)}\right\}
$$

onde $r=\sum_{i=1}^{k} r_{i}$ e $a_{1} \sum_{i=1}^{k} \frac{r_{i}}{V_{i}}$ 
A matriz de informação de Fisher com respeito a $\theta_{j}$ e $\beta$ é dada por,

$$
I\left(\theta_{j}, \beta\right)=\left[\begin{array}{cc}
\frac{r}{\theta_{j}^{2}} & \frac{b_{1 j}}{\theta_{j}} \\
\frac{b_{1 j}}{\theta_{j}} & b_{2 j}
\end{array}\right],
$$

onde $b_{1 j}=\sum_{i=1}^{k} r i\left(\frac{1}{V_{j}}-\frac{1}{V_{i}}\right)$ e $b_{2 j}=\sum_{i=1}^{k} r i\left(\frac{1}{V_{j}}-\frac{1}{V_{i}}\right)^{2}$.

Definindo $\overline{\bar{V}}_{j}=\frac{\sum_{i=1}^{k} r_{i}\left(\frac{1}{v_{j}}-\frac{1}{v_{i}}\right)}{\sum_{i=1}^{k} r_{i}}$, de forma análoga à sub-seção (2.6.4), e explorando o uso da reparametrização ortogonal (ver por exemplo, Cox e Reid, 1987) adotada para o parâmetro $\theta_{j}$, associada a reparametrização logarítmica adotada para o parâmetro ortogonal obtido, obtemos, $\theta_{j}=e^{\psi} e^{\beta \overline{\bar{V}_{j}}}$.

t.

Daí,

$$
L(\psi, \beta) \propto e^{-\psi r} \exp \left\{-e^{-\psi} \sum_{i=1}^{k} \frac{V_{i}}{V_{j}} A_{i} e^{\beta\left(\frac{1}{V_{j}}-\frac{1}{V_{i}}-\overline{\bar{V}}_{j}\right)}\right\}
$$

e a matriz de informação de Fisher com respeito a $\psi$ e $\beta$ é dada por,

$$
I(\psi, \beta)=\left[\begin{array}{ll}
r & 0 \\
0 & e_{j}
\end{array}\right]
$$

onde $e_{j}=\sum_{i=1}^{k} r_{i}\left(\frac{1}{V_{j}}-\frac{1}{V_{i}}-\overline{\bar{V}}_{j}\right)^{2}$

A densidade a priori não informativa de Jeffreys para $\psi$ e $\beta$ é dada por,

$$
\pi(\psi, \beta) \propto \text { constante }
$$

onde $-\infty<\psi, \beta<\infty$.

A densidade a posteriori para $\psi$ e $\beta$ é dada (de (10.6) e (10.4)) por, 


$$
\pi(\psi, \beta / \text { dados }) \propto e^{-\psi r} \exp \left\{-e^{-\psi} \sum_{i=1}^{k} \frac{V_{i}}{V_{j}} A_{i} e^{\beta\left(\frac{1}{V_{j}}-\frac{1}{V_{i}}-\overline{\bar{V}}_{j}\right)}\right\}
$$

onde $-\infty<\psi, \beta<\infty$.

De forma análoga a desenvolvida na seção 3.6 , temos que a densidade a posteriori para $\theta_{j}$ é dada por,

$$
\left.\begin{array}{c}
\pi\left(\theta_{j} / \text { dados }\right)=\frac{\left\{\sum_{i=1}^{k} \frac{v_{i}}{V_{j}} A_{i} e^{\bar{\beta}^{\prime}\left(\frac{1}{V_{j}}-\frac{1}{v_{i}}\right)}\right\}^{1 / 2}}{\Gamma(r-1 / 2)} \theta_{j}^{(r+1 / 2)} \cdot \\
\cdot \exp \left\{-\theta_{j}^{-1} \sum_{i=1}^{k} \frac{V_{i}}{V_{j}} A_{i} e^{\tilde{\beta}^{\prime}\left(\frac{1}{v_{j}}-\frac{1}{V_{i}}\right)}\right\}
\end{array}\right\}
$$

onde $\theta_{j}>0$ e $\tilde{\beta}^{\prime}$ é a moda da densidade a posteriori marginal para $\beta$ dada (de (10.7)) por,

$$
\pi(\beta / \text { dados }) \propto\left\{\sum_{i=1}^{k} V_{i} A_{i} e^{\beta\left(\frac{1}{V_{j}}-\frac{1}{V_{i}}-\overline{\bar{V}}_{j}\right)}\right\}^{-T}
$$

onde $-\infty<\beta<\infty$.

A densidade preditiva de uma observação futura $T_{(n+1) j}$ sob o nível de estresse $V_{j}$ é dada por (ver por exemplo, Aitchinson e Dunsmore, 1975; Press, 1989),

$$
f^{j *}\left(t_{(n+1) j} / \text { dados }\right)=\int_{0}^{\infty} f^{j}\left(t_{(n+1) j} / \theta_{j}\right) \pi\left(\theta_{j} / \text { dados }\right) d \theta_{j}
$$

onde $f^{j}\left(t_{(n+1) j} / \theta_{j}\right)=\theta_{j}^{-1} \exp \left\{-\theta_{j}^{-1} t_{(n+1) j}\right\}$, para $t_{(n+1) j} \geq 0$, e $\pi\left(\theta_{j} /\right.$ dados $)$ é dada em $\left(10.8^{\circ}\right)$, isto é, 


$$
f^{j *}\left(t_{(n+1) j} / \text { dados }\right)=\frac{(r-1 / 2) V_{j} e^{-\tilde{\beta}^{\prime} / V_{j}}\left\{\sum_{i=1}^{k} V_{i} A_{i} e^{-\tilde{\beta}^{\prime} / V_{i}}\right\}^{r-1 / 2}}{\left\{t_{(n+1) j} V_{j} e^{-\tilde{\beta}^{\prime} / V_{j}}+\sum_{i=1}^{k} V_{i} A_{i} e^{-\tilde{\beta}^{\prime} / V_{i}}\right\}^{r+1 / 2}}
$$

onde $t_{(n+1) j}>0$ (densidade de Pareto).

Considerando a densidade preditiva (10.11) temos que,

$$
P^{j 4}\left(T_{(n+1) j}>L_{j}^{*} / \text { dados }\right)=(r-1 / 2)\left(\frac{c e^{\bar{\beta}^{\prime} / V_{j}}}{V_{j}}\right)^{r-1 / 2} \int_{L_{j}^{*}}^{\infty} \frac{d t_{(n+1) j}}{\left\{\frac{c e^{\beta^{\prime} / V_{j}}}{V_{j}}+t_{(n+1) j}\right\}^{r+1 / 2}}
$$

onde $c=\sum_{i=1}^{k} V_{i} A_{i} e^{\tilde{\beta}^{\prime} / V_{i}}$.

Fixando essa probabilidade em $\left(1-\gamma^{\prime}\right)$ obtemos,

$$
\frac{\left(\frac{c e^{\tilde{\beta}^{\prime} / V_{j}}}{V_{j}}\right)^{r-1 / 2}}{\left(L_{j}^{*}+\frac{c e^{\dot{e}^{\prime} / V_{j}}}{V_{j}}\right)^{r-1 / 2}}=1-\gamma^{\prime}
$$

De (10.13), como $V_{j}$ é fixo, achamos $L_{j}^{*}$, dado por,

$$
L_{j}^{*}=\frac{c\left[1-\left(1-\gamma^{\prime}\right)^{\frac{1}{r^{-1 / 2}}}\right]}{V_{j} e^{-\tilde{\beta}^{\prime} / V_{j}}\left(1-\gamma^{\prime}\right)^{\frac{1}{r-1 / 2}}},
$$

onde $\hat{\beta}^{\prime}$ é a moda da densidade a posteriori (10.9).

Portanto, um procedimento de controle de qualidade pode ser adotado a partir de (10.14).

Como exemplo, verificamos que considerando os dados da Tabela $9.1 \mathrm{e}$ assumindo $j=1, V_{1}=10,1-\gamma^{\prime}=0,80$, a moda da densidade a posteriori marginal 
(10.0) é dada por $\tilde{\beta}^{\prime}=5,20525$, e o período de tempo requerido em um teste de cortrole de qualidade é dado (de (10.14)) por, $L_{j}^{*}=833,293$, próximo do obtido considerando-se $\beta$ conhecido, dado (ver Tabela 9.5) por, 814, 297.

\section{Outros Tópicos de Interesse}

Observe que, no Capítulo 2, em nenhum momento, mencionamos as possíveis interrelações e equivalências entre as reparametrizações adotadas.

Neste contexto, poderiamos nos ater, por exemplo, a mostrar que a função de verossimilhança relativa maximizada para o tempo médio de sobrevivência, $\theta_{1}$, sob o nível usual de funcionamento é invariante sob a reparametrização ortogonal (ver por exemplo, Cox e Reid, 1987) adotada para o parâmetro $\beta$, como pode ser visto em Louzada Neto e Rodrigues (1991) que analisam, sobre esse enfoque, o modelo de Potência. Isto pode ser considerado um estudo que futuramente poderá ser desenvolvido.

Outro tópico de interesse é o uso de planejamentos fatoriais (ver por exemplo, Box, Hunter e Hunter, 1978) em testes de sobrevivência acelerados no enfoque Bayesiano. Análises de testes acelerados considerando o uso de planejamento fatoriais, no enfoque clássico de análise, podem ser encontrados em Mann, Schaffer e Singpurwalla (1974).

Considerar uma Análise Bayesiana com outras distribuições para os tempos de sobrevivência dos componentes submetidos a um teste acelerado, como por exemplo, a Gamma, e distribuições mistas, como por exemplo, Weibull - Exponencial - Weibull, assumindo a relação estresse-resposta geral (6.1), consiste em um outro tópico de pesquisa futura. Além disso, considerar a análise da confiabilidade de sistemas em séries e paralelo (ver por exemplo, Bain, 1978). 
Um estudo detalhado de diferentes parametrizações em testes de sobrevivência acelerados no enfoque Bayesiano poderá ser considerado (além do incluido nesta dissertação), incluindo seu uso em controle de qualidade.

Possivelmente, poderemos obter resultados de forma similar aos obtidos nesta dissertação (usando o método de Laplace para aproximação de integrais) considerando diferentes densidades a priori, em particular, densidades a priori informativas.

Finalmente, temos interesse no desenvolvimento de um software de fácil acesso, que operacionaliza a análise de testes de sobrevivência acelerados, no estudo da confiabilidade de um determinado componente manufaturado. 


\section{Apêndice 1}

\section{O Método "Delta"}

O método "Delta" pode ser encontrado em vários textos de inferência (ver por exemplo, Miller, 1981) e é definido da seguinte forma.

Supor que $Y$ é uma variável aleatória com média $\mu$ e variância $\sigma^{2}$ e que estamos interessados em determinar a distribuição de alguma função $g(Y)$ contínua de $Y$.

Expandindo $g(Y)$ ao redor de $\mu$, por uma série de Taylor,

$$
g(Y)=g(\mu)+(Y-\mu) \frac{\partial}{\partial Y} g(Y)+\ldots
$$

e desconsiderando os termos de ordens mais elevadas ( $>1)$,

$$
E[g(Y)] \cong g(\mu)
$$

e

$$
\sigma_{g}^{2} \cong \sigma^{2}\left(\frac{\partial g}{\partial Y}\right)^{2}
$$

Além disso, se $Y \stackrel{a}{\sim} N\left(\mu, \sigma^{2}\right)$, então

$$
g(Y) \stackrel{a}{\sim} N\left(g(\mu) ; \sigma_{g}^{2}\right)
$$


(ver pnr exemplo, Leite e Singer, 1990 - Teorema 3.3.2).

Em sua versão multivariada, supor $X$ e $Y$ duas variáveis aleatórias com médias $\mu_{X}$ e $\mu_{Y}$ e variâncias $\sigma_{X}^{2}$ e $\sigma_{Y}^{2}$, respectivamente, e covariância $\sigma_{X Y}$, e que, também, desejamos a distribuição de $g(X, Y)$ contínua em $X$ e $Y$.

Expandindo $g(X, Y)$ ao redor de $\left(\mu_{X}, \mu_{Y}\right)$, por uma série de Taylor,

$$
\begin{aligned}
g(X, Y) & =g\left(\mu_{X}, \mu_{Y}\right)+\left(X-\mu_{X}\right) \frac{\partial}{\partial X} g\left(\mu_{X}, \mu_{Y}\right)+ \\
& +\left(Y-\mu_{Y^{\prime}}\right) \frac{\partial}{\partial Y} g\left(\mu_{X}, \mu_{Y}\right)+\ldots
\end{aligned}
$$

e desconsiderando os termos de ordens mais elevadas $(>1)$,

$$
E[g(X, Y)] \cong g\left(\mu_{X}, \mu_{Y}\right)
$$

e

$$
\begin{gathered}
\sigma_{g}^{2} \cong \sigma_{X}^{2}\left(\frac{\partial g}{\partial X}\right)^{2}+\sigma_{Y}^{2}\left(\frac{\partial g}{\partial Y}\right)^{2}+2 \sigma_{X Y}\left(\frac{\partial g}{\partial X}\right)\left(\frac{\partial g}{\partial Y}\right) \\
\operatorname{Se}(X, Y) \stackrel{a}{\sim} N\left\{\left(\mu_{X}, \mu_{Y}\right) ;\left[\begin{array}{cc}
\sigma_{X}^{2} & \sigma_{X Y} \\
\sigma_{X Y} & \sigma_{Y}^{2}
\end{array}\right]\right\}, \quad \text { então } \\
\qquad g(X, Y) \stackrel{a}{\sim} N\left\{g\left(\mu_{X}, \mu_{Y}\right) ; \sigma_{g}^{2}\right\}
\end{gathered}
$$

(ver por exemplo; Leite e Singer, 1990 - Teorema 3.3.4). 


\section{Apêndice 2 \\ O Método de Aproximação de La- place para Integrais}

Uma das limitações dos métodos Bayesianos nas aplicações em geral relacionase à resolução de integrais que muitas vezes não apresentam soluções analíticas explícitas e exigem o uso de métodos de aproximação.

Neste sentido, um dos métodos mais utilizados em inferência Bayesiana aproximada é o Método de Laplace para aproximação de integrais (ver por exemplo, Tierney e Kadane, 1986; Kass, Tierney e Kadane, 1990).

Supor que estamos interessados em resolver a integral em $\theta$, dada por,

$$
\int f(\theta) e^{-n h(\theta)} d \theta
$$

No caso uniparamétrico $(\theta \in \mathrm{R})$ se $\hat{\theta}$ é o valor que maximiza $-n h(\theta)$ em (A2.1), $f(\theta)$ é uma função monótona positiva e $\sigma=\left\{n h^{\prime \prime}(\hat{\theta})\right\}^{\frac{1}{2}}$, então o método de aproximação de Laplace é dado por (ver por exemplo, Kass, Tierney e Kadane, 1990),

$$
\int f(\theta) e^{-n h(\theta)} d \theta \cong \sqrt{2 \pi} \sigma f(\hat{\theta}) e^{-n h(\hat{\theta})}
$$

No caso multiparamétrico, $\operatorname{com} \theta \in \mathbf{R}^{m}$, a aproximação de Laplace para (A2.1) é dada por, 


$$
\int f(\theta) e^{-n h(\theta)} d \theta \cong(2 \pi)^{\frac{m}{2}}\left|n D^{2} h(\hat{\theta})\right|^{\frac{1}{2}} f(\hat{\theta}) e^{-n h(\hat{\theta})}
$$

onde $\hat{\theta}$ maximiza $-n h(\theta), D^{2} h(\hat{\theta})$ é a matriz Hessiana calculada em $\hat{\theta}$.

Em geral, em inferência Bayesiana, o interesse é inferir sobre um determinado parâmetro, e a obtenção de sua densidade a posteriori marginal, através de integração, se faz necessária.

Supor que estamos interessados em obter a densiade a posteriori de um parâmetro $\phi$ na presença de parâmetro "nuisance" $\gamma$, isto é,

$$
\pi(\phi / d a d o s)=\int \pi(\phi, \gamma / \text { dados }) d \gamma=\int \pi(\phi, \gamma) L(\phi, \gamma) d \gamma
$$

onde $\pi(\phi, \gamma)$ é a densidade a priori para $\phi$ e $\gamma$ e $L(\phi, \gamma)$ é a função de verossimilhança para $\phi$ e $\gamma$.

Considerando,

$$
f(\gamma) e^{-n h_{\phi}(\gamma)}=\pi(\phi, \gamma) L(\phi, \gamma)
$$

a densidade a posteriori marginal para $\theta$ pode ser escrita na forma,

$$
\pi(\phi / d a d o s)=\int f(\gamma) e^{-n h_{\phi}(\gamma)} d \gamma
$$

Neste caso, o métođo de Laplace para aproximação de integrais é dado por (ver por exemplo, Kass, Tierney e Kadane, 1990),

$$
\int f(\gamma) e^{-n h_{\phi}(\gamma)} d \gamma \cong f(\hat{\gamma})\left|-n h_{\phi}^{\prime \prime}(\hat{\gamma})\right|^{-\frac{1}{2}} e^{-n h_{\phi}(\hat{\gamma})}
$$


de $\hat{\gamma}$ maximiza $-n h_{\phi}(\gamma)$ para cada valor fixo de $\phi \mathrm{e}-n h_{\phi}^{\prime \prime}(\hat{\gamma})$ é a segunda derivada de $-n h_{\phi}(\gamma)$ com respeito a $\gamma$ calculada em $\hat{\gamma}$.

Sem perda de generalidade, definindo,

$$
f(\gamma)=\mathbb{I},
$$

e

$$
-n h_{\theta}(\gamma)=\log (\pi(\phi, \gamma)) \ell(\phi, \gamma),
$$

onde II é a matriz indentidade e $\ell(\phi, \gamma)=\log (L(\phi, \gamma))$, podemos escrever (A2.4) na forma,

$$
\because(\phi / \text { dados })=\int e^{-n h_{\phi}(\gamma)} d \gamma .
$$

O método de Laplace, neste caso, é dado (ver por exemplo, Tierney e Kadane, 1986) por,

$$
\int e^{-n h_{\phi}(\gamma)} d \gamma \cong\left|-n h_{\phi}^{\prime \prime}(\hat{\gamma})\right|^{\frac{1}{2}} e^{-n h_{\phi}(\hat{\gamma})}
$$

Quando $f()=$. II chamaremos o método de Laplace por método de Laplace completamente exponencial.

Devemos ressaltar que essa aproximação (A2.10) no caso multiparamétrico é obtida de forma análoga.

Maiores detalhes e estudo sobre a precisão da metodologia descrita acima podem ser encontrados em Tierney e Kadane, (1986); Kass, Tierney e Kadane, 
(1990), Achcar $(1988,1989)$ e Achcar e Smith (1990d). Em Bolfarine, Rodrigues e Achcar (1991) encontramos aplicações do método de Laplace completamente exponencial em modelos de sobrevivência.

\section{Apêndice 2.1}

\section{Cálculos para Obtenção da Densidade a Posteriori Marginal para o Parâmetro de forma $p$}

A densidade a posteriori marginal para $p$ é dada (de (5.13)) por,

$$
\pi(p / \text { dados }) \propto p^{r+1}\left(\prod_{i=1}^{k} \prod_{j=1}^{r_{i}} t_{i j}^{* p-1}\right)\left(\prod_{i=1}^{k} V_{i}^{p r_{i}}\right) \int_{-\infty}^{\infty} \int_{-\infty}^{\infty} e^{-n h_{p}(\alpha, \beta)} d \alpha d \beta
$$

onde $-n h_{p}(\alpha, \beta)=p \alpha r-p \beta a_{1}-e^{p \alpha} \sum_{i=1}^{k} V_{i}^{p} A_{i}(p) e^{-\beta p / V_{i}}$.

Para resolver esta integral usamos o método de Laplace completamente exponencial no caso multiparamétrico (ver Apêndice 2), isto é,

$$
\int_{-\infty}^{\infty} \int_{-\infty}^{\infty} e^{-n h_{p}(\alpha, \beta)} d \alpha d \beta \cong(2 \pi)^{\frac{m}{2}}\left\{\operatorname{det}\left(n \mathbf{D}^{2} h_{p}(\hat{\alpha}, \hat{\beta})\right\}^{-\frac{1}{2}} e^{-n h_{p}(\hat{\alpha}, \hat{\beta})}\right.
$$

onde o par $(\hat{\alpha}, \hat{\beta})$ maximiza $-n h_{p}(\alpha, \beta)$ e $\mathbf{D}^{2} h_{p}(\hat{\alpha}, \hat{\beta})$ é a matriz Hessiana de $h_{p}(\alpha, \beta)$ calculada em $(\hat{\alpha}, \hat{\beta})$.

As primeiras derivadas de $-n h_{p}(\alpha, \beta)$ são dadas por,

$$
\left.\begin{array}{l}
-\frac{\partial n h_{p}(\alpha, \beta)}{\partial \alpha}=p r-p e^{p \alpha} \sum_{i=1}^{k} V_{i}^{p} A_{i}(p) e^{-\beta p / V_{i}}, \\
-\frac{\partial n h_{p}(\alpha, \beta)}{\partial \beta}=-p a_{1}+p e^{p \alpha} \sum_{i=1}^{k} V_{i}^{p-1} A_{i}(p) e^{-\beta p / V_{i}}
\end{array}\right\}
$$


Igualando as primeiras derivadas (A2.1.2) a zero, o máximo de $-n h_{p}(\alpha, \beta)$ é dado por $\hat{\alpha}$ e $\hat{\beta}$ que satisfazem:

$$
\left.\begin{array}{l}
r=e^{p \hat{\alpha}} \sum_{i=1}^{k} V_{i}^{p} A_{i}(p) e^{-\hat{\beta} p / V_{i}}, \\
a_{2}=e^{p \hat{\alpha}} \sum_{i=1}^{k} V_{i}^{p-1} A_{i}(p) e^{-\hat{\beta} p / V_{i}}
\end{array}\right\}
$$

Portanto, $\hat{\beta}$ é a solução da equação,

$$
\frac{r}{a_{1}}=\frac{\sum_{i=1}^{k} V_{i}^{p} A_{i}(p) e^{-\hat{\beta}_{p} / V_{i}}}{\sum_{i=1}^{k} V_{i}^{p-1} A_{i}(p) e^{-\hat{\beta} p / V_{i}}}
$$

e

$$
\hat{\alpha}=\frac{1}{p} \log \left\{\frac{r}{\sum_{i=1}^{k} V_{i}^{p} A_{i}(p) e^{-\hat{\hat{\beta}_{p}} / V_{i}}}\right\} .
$$

Observe que para cáda valor fixo de $p$ podemos achar $\beta$ que maximiza $-n h_{p}(\hat{\alpha}, \beta)$ onde $\hat{\alpha}$ é dado em (A2.1.5), isto é, substituindo (A2.1.5) em $-n h_{p}(\alpha, \beta)$, dado em (A2.1.1) podemos definir,

$$
g(\beta)=-n h(\hat{\alpha}, \beta)=r \log \left\{\frac{r}{\sum_{i=1}^{k} V_{i}^{p} A_{i}(p) e^{-\beta p / V_{i}}}\right\}-p \beta a_{1}-r
$$

Assim, para cada valor fixo de $p$ achamos $\hat{\beta}$ que maximiza $g(\beta)$ e daí, $\epsilon \in$ ncontramos $\hat{\alpha}$ a partir de (A2.1.5).

Agora, devemos obter o $\operatorname{det}\left\{n \mathbf{D}^{2} h_{p}(\hat{\alpha}, \hat{\beta})\right\}$.

As segundas derivadas de $-n h_{p}(\alpha, \beta)$ são dadas por, 


$$
\left.\begin{array}{c}
\frac{\partial^{2} n h(\alpha, \beta)}{\partial \alpha^{2}}=p^{2} e^{p \alpha} \sum_{i=1}^{k} V_{i}^{p} A_{i}(p) e^{-\beta p / V_{i}}, \\
\frac{\partial^{2} n h(\alpha, \beta)}{\partial \beta^{2}}=p^{2} e^{p \alpha} \sum_{i=1}^{k} V_{i}^{p-2} A_{i}(p) e^{-\beta p / V_{i}}, \\
\frac{\partial^{2} n h(\alpha, \beta)}{\partial \alpha \partial \beta}=-p^{2} e^{p \alpha} \sum_{i=1}^{k} V_{i}^{p-1} A_{i}(p) e^{-\beta p / V_{i}}
\end{array}\right\}
$$

Observe que,

$$
\begin{gathered}
\left.\frac{\partial^{2} n h(\hat{\alpha}, \hat{\beta})}{\partial \alpha^{2}}=p^{2} e^{p \hat{\alpha}} \sum_{i=1}^{k} V_{i}^{p} A_{i}(p) e^{-\hat{\beta}_{p} / V_{i}}=r p^{2} \text { por }(\mathrm{A} 2.1 .3)\right) \\
\frac{\partial^{2} n h(\hat{\alpha}, \hat{\beta})}{\partial \beta^{2}}=p^{2} e^{p \hat{\alpha}} \sum_{i=1}^{k} V_{i}^{p-2} A_{i}(p) e^{-\hat{\beta} p / V_{i}}
\end{gathered}
$$

e

$\left.\frac{\partial^{2} n h(\hat{\alpha}, \hat{\beta})}{\partial \alpha \partial \beta}=-p^{2} e^{p \hat{\alpha}} \sum_{i=1}^{k} V_{i}^{p-1} A_{i}(p) e^{-\hat{\beta}_{p} / V_{i}}=-a_{1} p^{2}(\text { por }(\mathrm{A} 2.13))_{j}\right)$

Dessa forma,

$$
\operatorname{det}\left\{n \mathrm{D}^{2} h_{p}(\hat{\alpha}, \hat{\beta})\right\}=p^{4}\left\{r^{2} \frac{\sum_{i=1}^{k} V_{i}^{p-2} A_{i}(p) e^{-\hat{\beta} p / V_{i}}}{\sum_{i=1}^{k} V_{i}^{p} A_{i}(p) e^{-\hat{\beta} p / V_{i}}}-a_{1}^{2}\right\}
$$

Considerando as expressões (A2.1.1), (A2.1.6) e (A2.1.8), a densidade a posteriori marginal para $p$ aproximada pelo método de Laplace completamente exponencial, é dada por,

$$
\pi(p / \text { dados }) \propto \frac{p^{r-1} e^{-p a_{1} \bar{\beta}}\left(\prod_{i=1}^{k} \prod_{j=1}^{r_{i}} t_{i j}^{* p-1}\right)\left(\prod_{i=1}^{k} V_{i}^{p r_{i}}\right)}{\left\{r^{2} \frac{\sum_{i=1}^{k} V_{i}^{p-2} A_{i}(p) e^{-\hat{\beta}_{p} / V_{i}}}{\sum_{i=1}^{k} V_{i}^{p} A_{i}(p) e^{-\dot{\beta}_{p} / V_{i}}}-a_{1}^{2}\right\}^{\frac{1}{2}}\left\{\sum_{i=1}^{k} V_{i}^{p} A_{i}(p) e^{-\hat{\beta} p / V_{i}}\right\}^{r}}
$$


onde $p>0$ e $\hat{\beta}$ é $\circ$ valor que mazimiza $g(\beta)=$ $r \log \left\{\frac{r}{\sum_{i=1}^{k} v_{i}^{p} A_{i}(p) e^{-\hat{\beta} p / V_{i}}}\right\}-p \beta a_{1}-r$, para cada valor fixo de $p$ (obtemos assim, a expressão (5.15)). 


\section{Apêndice 3}

\section{Teoria da Verossimilhança: Regiões e Intervalos de Verossimilhança}

Neste apêndice apresentamos, resumidamente, uma técnica para obtenção de intervalos de confiança totalmente baseada na função de verossimilhança para os parâmetros envolvidos.

Supor uma determinada função de probabilidade que envolve dois parâmetros desconhecidos $\phi$ e $\gamma$, e que, somente, $\phi$ é o parâmetro de interesse.

A função de verossimilhança relativa para $\phi$ e $\gamma$ é dada por (ver por exemplo, Kalbfleisch, 1985),

$$
R(\phi, \gamma)=\frac{L(\phi, \gamma)}{L(\hat{\phi}, \hat{\gamma})}
$$

onde $L(\phi, \gamma)$ é a função de verossimilhança para $\phi$ e $\gamma$ e $L(\hat{\phi}, \hat{\gamma})=\sup _{\phi, \gamma} L(\phi, \gamma)$, com o par $(\hat{\phi}, \hat{\gamma})$ estimadores de máxima verossimilhança para $(\phi, \gamma)$.

A função de verossimilhança relativa maximizada para $\phi$ é dada por,

$$
R_{\gamma}(\phi)=\max _{\gamma} R(\phi, \gamma)=R(\phi, \hat{\gamma}(\phi))
$$

O conjunto de valores de $\phi$ para os quais $R_{\gamma}(\phi) \geq p$ é chamado de região de verosşimilhança $100 \mathrm{p} \%$ para $\phi$. Usualmente, uma região de verossimilhança $100 \mathrm{p} \%$ consiste de um interalo de valores reais chamado de Intervalo de Verossimilhança $100 \mathrm{p} \%$ para $\phi$.

$\mathrm{Na}$ maioria dos casos, usamos Intervalos de Verossimilhança com $p=$ 
$0,5, p=0,10 e p=0,01$, isto é Intervalos de Verossimilhança $50 \%, 10 \%$ e $1 \%$ para $\phi$. Valores dentro dos Intervalos de Verossimilhança $100 \mathrm{p} \%$ são considerados mais prováveis para $\phi$, segundo os dados obtidos em um determinado experimento e um nível especificado $100 \mathrm{p} \%$.

Intervalos de verossimilhança $100.0,147 \%$ para $\phi$ são muitas vezes calculados, que são, aproximadamente, intervalos de confiança $95 \%$ para $\phi$ (ver por exemplo, Kalbfleisch, 1985).

Um intervalo aleatório $\left[\phi_{*}, \phi^{*}\right]$ é chamado de intervalo de confiança para $\phi$ se sua Probabilidade de Cobertura (PC) é dada por,

$$
P C\left(\phi_{0}\right)=P\left(\phi_{*} \leq \phi_{0} \leq \phi^{*} / \phi=\phi_{0}\right)
$$

para todos os valores possiveis de $\phi_{0}$.

A Probabilidade de Cobertura (PC) do intervalo de confiança é o chamado coeficiente de confiança.

Portanto, particularmente, um intervalo de confiança $95 \%$ para $\phi$ é dado por,

$$
P\left(\phi_{*} \leq \phi_{0} \leq \phi^{*} / \phi=\phi_{0}\right)=0,95
$$

para todos os possiveis valores de $\phi_{0}$.

Agora, definindo,

$$
r_{\gamma}(\phi)=\log \left(R_{\gamma}(\phi)\right)
$$


observamos que,

$$
-2 r_{\gamma}(\phi) \stackrel{a}{\sim} \chi_{(1)}^{2}
$$

e os Intervalos de Verossimilhança para $\phi$ são exatamente ou aproximadamente os intervalos de confiança para $\phi$; e o coeficiente de confiança de um intervalo de verrossimilhança é dado por,

$$
P C \cong P\left\{\chi_{(1)}^{2} \leq-2 \log p\right\}
$$

Dai, com $p=0,147, P\left\{\chi_{(1)}^{2} \leq 2 \log (0,147)\right\}=P\left\{\chi_{(1)}^{2} \leq 2(-1,92)\right\}=$ 0,95 .

Fazendo um gráfico de $\phi$ versus $r_{\gamma}(\phi)$, determinamos graficamnete um intervalo de verossimilhança $100.0,147 \%$ aproximade para $\phi$, isto é, um intervalo de confiança aproximado $95 \%$ para $\phi$ é obtido traçando-se uma reta em $r_{\gamma}(\phi)=-1,92$, como na Figura A3.1. Podemos notar que o mesmo intervalo pode ser obtido construindo-se o gráfico de $\phi$ versus $R_{\gamma}(\phi)$ e traçando-se uma reta em $R_{\gamma}(\phi)=0,147$ (ver por exemplo, Kalbfleisch, 1985).

Um estudo mais detalhado sobre o método descrito acima pode ser encontrado em Kalbfleisch (1985). Mann, Schaffer e Singpurwalla, (1974), Barbosa e Louzada Neto (1991a) apresentam aplicações desta metodologia em testes de sobrevivência acelerado. 


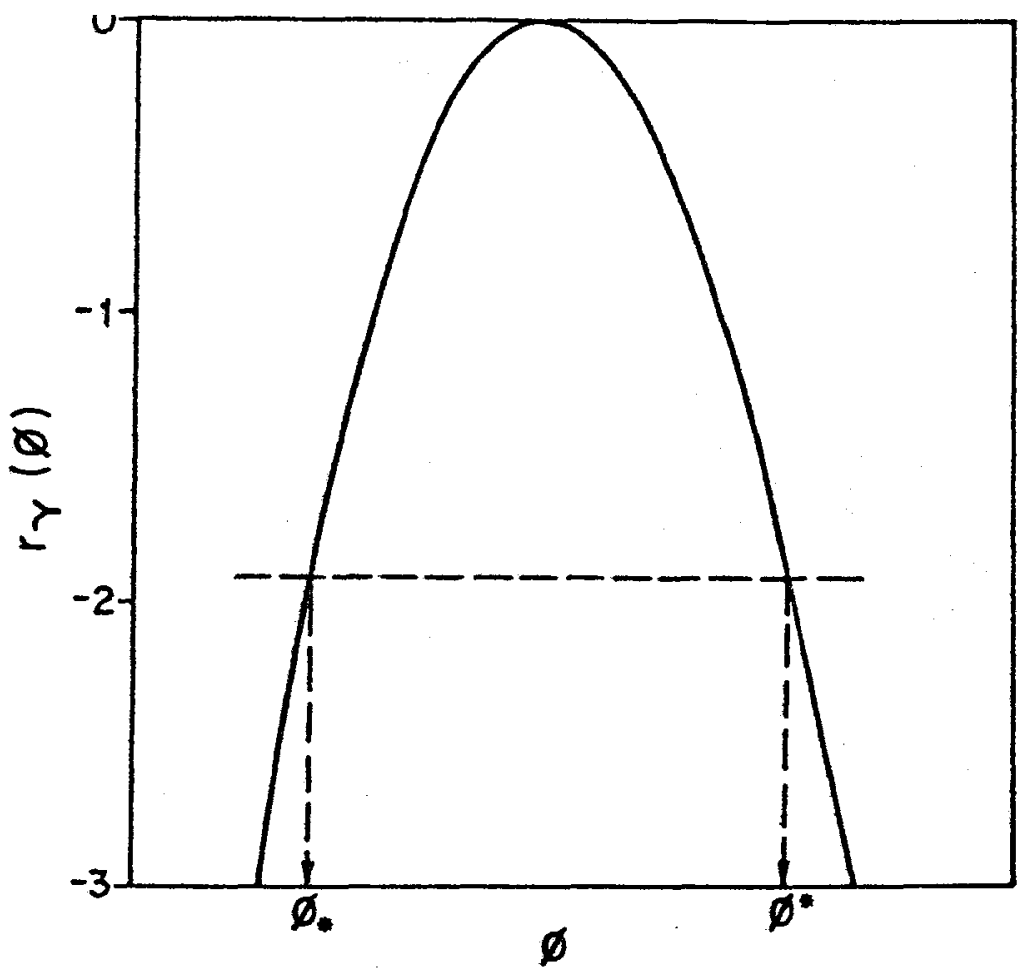

Figura A3.1: Gráfico de $\phi$ versus $r_{\gamma}(\phi)$ com um Intervalo de Confiança Aproximado $95 \%$ para $\phi$ Dado Por $\left(\phi_{*}, \phi^{*}\right)$. 


\section{Apêndice 4}

\section{Obtenção de Intervalos HPD}

Em inferência Bayesiana, em geral temos interesse em determinar uma região $R$ (do espaço paramétrico) para a qual a probabilidade de conter a densidade a posteriori para um determinado parâmetro $\phi$ é $\left(1-\gamma^{\prime}\right)$.

Como discutido em Box e Tiao (1973), tal região é chama de Região "a Posteriori de maior Probabilidade " (HPD) 100(1- $\left.\gamma^{\prime}\right) \%$ se

$$
\int_{R} \pi(\phi / \text { dados }) d \phi=1-\gamma^{\prime}
$$

onde $\pi(\phi /$ dados $)$ é a densidade a posteriori para $\phi$, e

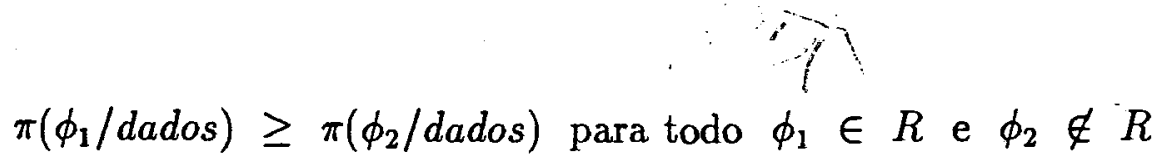

(ver por exemplo, Martz e Waller, 1982).

Em muitos problemas práticos existem dificuldades na determinação de $R$. Porém, como no Apêndice 3, podemos definir, de forma análoga, a "densidade a posteriori relativa para $\phi^{\prime \prime}$, dada por

$$
R=R(\phi / \text { dados })=\frac{\pi(\phi / \text { dados })}{\pi(\tilde{\phi} / \text { dados })}
$$

onde $\pi(\phi /$ dados $)$ é definida em (A4.1) e $\pi(\tilde{\phi} /$ dados $)$ é o valor da densidade a posteriori para $\phi$ calculada em $\tilde{\phi}(\tilde{\phi}$ é a moda a posteriori para $\phi)$; e o conjunto de valores de $\phi$ tais que $R \geq \phi^{\prime}$ é chamado de intervalo HPD para $\phi$. 
Considerando a aproximação $\chi^{2}$, isto é, $-2 \log R \stackrel{a}{\sim} \chi_{(1)}^{2}$,

$$
1-\gamma^{\prime} \approx P\left(\chi_{(1)}^{2} \leq-2 \log \phi^{\prime}\right)
$$

Portanto, se $\phi^{\prime}=0,147, P\left(\chi_{(1)}^{2} \leq 2(-1,92)\right) \approx 0,95$, e um intervalo HPD aproximado $95 \%$ para $\phi$ pode ser obtido graficamente contruindo-se o gráfico de $\phi$ versus $R$ e traçando-se uma reta em $R=0,147$ como na Figura A4.1.

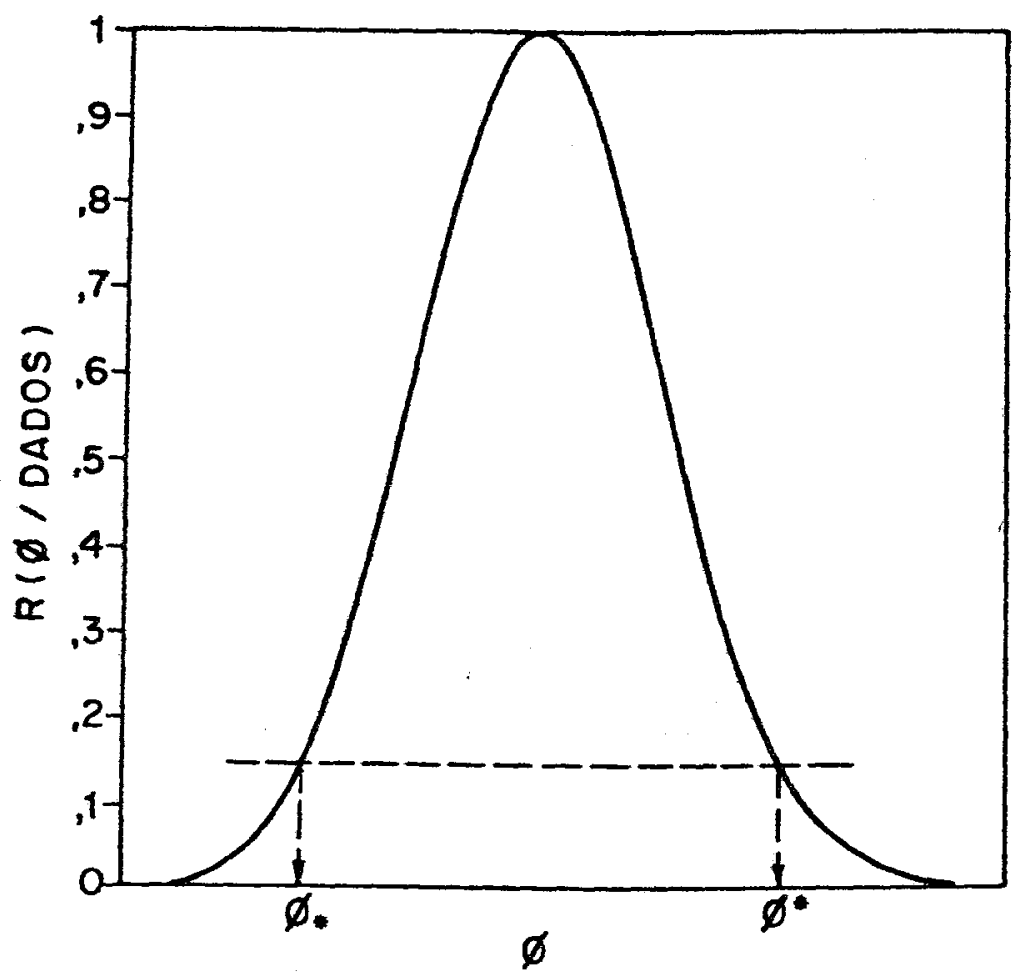

Figura A4.1: Gráfico de $\phi$ versus $R(\phi /$ dados $)$ com um intervalo HPD aproximado $95 \%$ para $\phi$, dado por, $\left(\phi_{*}, \phi^{*}\right)$. 


\section{Bibliografia}

ACHCAR, J.A. Alguns Aspectos de Inferência Bayesiana Aproximada. São Carlos, ICMSC-USP, 1988, 94 p. (Tese de Livre Docência).

ACHCAR, J.A. Reparametrizations and Laplace Aproximations for Posterior Moments in Binomial Models. Revista de Matemática e Estatística, 7, 73-86, 1989.

ACHCAR, J.A. Use of Predictive Densities in Quality Control with Accelerated Life Tests Assuming a Power Rule Model and an Exponential Distribution. São Carlos, ICMSC-USP, 1990a, s.p. (Notas do ICMSC-USP, 76).

ACHCAR, J.A.; DIAS, T.C.D. Accelerated Life Tests with an Exponential Distribution: A Bayesian Approach with the Power Rule Model and Type II Censored Data. São Carlos, ICMSC-USP, 1990b, s.p. (Notas do ICMSC-USP, $67)$.

ACHCAR, J.A.; LOUZADA NETO, F. Accelerated Life Tests with an Exponential Distribution: A Bayesian Approach with the Generalized Eyring Model and Type II Censored Data. São Carlos, ICMSC-USP, 1990c, s.p. (Notas do ICMSC-USP, 73).

ACHCAR, J.A.; LOUZADA NETÓ, F. Accelerated Life Tests with one Stress Variable: A Bayesian Analysis of the Eyring Model. São Carlos, ICMSC-USP, 1991a, s.p. (Notas do ICMSC-USP, 85). 
ACHCAR, J.A.; LOUZADA NETO, F. A Baysian Approach for Accelerated Life Tests Considering the Weibull Distribution. São Carlos, ICMSC-USP, 1991b, s.p. (Notas do ICMSC-USP, 99).

ACHCAR, J.A.; SMITH, A.F.M. Aspects of Reparametrization in Approximate Bayesian Inference. Bayesian and Likelihood Methods in Statistics and Econometrics. North-Holland, Elsevier Science Publishers B.V., 1990d.

ACHCAR, J.A.; ROSALES, O.L.A. A Bayesian Approach for Accelerated Life Tests Assuming an Inverse Gaussian Distribution. São Carlos, ICMSC-USP, 1991c, 19 p. (Notas do ICMSC-USP, 94).

AITCHINSON, J.; DUNSMORE, I.R. Statistical Prediction Analysis. Cambridge University Press, 1975.

BAIN, L.J. Statistical Analysis of Reliability and Life-Testing Models. New York, Dekker, 1978.

BARBOSA, E.P.; LOUZADA NETO, F. Análise de Experimentos de Testes de Vida Acelerados com Distribuição de Falhas Exponencial via Modelos Lienares Generalizados. 1991a. (Submetido).

BARBOSA, E.P.; LOUZADA NETO, F. Analysis of Accelerated Life Test via Generalized Linear Modelling in the Case of a Weibull Failure Distribution and Stress-Response Relationship. 1991b. (Submetido).

BASU, A.P.; EBRAHIMI, N. Nonparametric Accelerated Life Testing. IEEE Transactions on Reliability, R-31, 5, 432-435, 1982.

BICKEL, P.J.; DUKSUM, K.A. Mathematical Statistics - Basic Ideas and Selected Topics. San Francisco, Holden-Day, 1977. 
BOLFARINE, H.; RODIGUES, J.; ACHCAR, J.A. Análise de Sobrevivência. Rio de Janeiro, IM-UFRJ, 1991, 112 p. (Notas do Minicurso da II Escola de Modelos de Regressão).

BOX, G.E.P.; HUNTER, W.G.; HUNTER, J.S. Statistics for Experimenters. New York, John Wiley \& Sons, 1978.

BOX, G.E.P.; TIAO, G.C. Bayesian Inference in Statistical Analysis. New York, Addison-Wesley, ${ }^{1} 1973$.

COX, D.R.; REID, N. Orthogonal Parameters and Approximated Conditional Inference with Discussion. Journal Royal Statistical Society B, 49, 1, 1-39, 1987.

DRAPER, N.R.; SMITH, H. Apllied Regression Analysis, 2a ed., New York, John Wiley \& Sons, 1981.

GOLDBERG. Comprehensive Failure Mechanisms Theory. Physics of Failure in Eletronics, 2a ed., 25-60, 1964.

HILLS, S.E. Parameters Tránsformations. Nottingham Statistics Group, Mathematics Departament, Nottingham University, 1987. (Relatório Técnico).

KALBFLEISCH, J.G. Probability and Statistical Inference - Vol 2: Statistical Inference. 2a ed., New York, Springer-Verlag, 1985.

KASS, R.E.; TIERNEY, L.; KADANE, J.B. The Validity of Posterior Expansions based on Laplace's Method, in Essays in Honor of George A. Barnard, Hồdger, Amsterdam, 1990.

KLEIN, J.P; BASU, A.P. Weibull Accelerated Life Tests when There are Competing Causes of Failure. Communications in Statistics. Part A: Theory and Methods, 10(20), 2073-2100, 1981. 
LAWLESS, J.F. Statistical Models and Methods for Lifetime Data. New York, Jonh Wiley \& Sons, 1982.

LEITE, J.G.; SINGER, J.M. Métodos Assintóticos em Estatística - Fundamentos e Aplicações. São Paulo, IME-USP, 1990, 130 p. (Notas do Mini-Curso do IX Simpósio Brasileiro de Probabilidade e Estatística).

LEVENBACH, G.J. Accelerated Life Testing of Capacitors. IRE Transactions, 10, 9-20, 1957.

LOUZADA NETO, F.; RODRIGUES, J. Reparametrização Ortogonal em Testes de Sobrevivência Acelerados com o Modelo de Potência. 1991. (Submetido).

MANN, N.R. Design of Over-Stress Life-Test Experiments when Failure Times Have the Two-Parameters Weibull Distribution. Technometrics, 14, 2, 437$451,1972$.

MANN, N.R.; SCHAFFER, R.E.; SINGPURWALLA, N.ע. Methods for Statistical Analysis of Reliability and Life Test Data. New York, John Wiley \& Sons, 1974.

MARTZ, H.F.; WALLER, R.A. Bayesian Reliability Analysis. New York, John Wiley \& Sons, 1982.

McCullagh, P.; Nelder, J. Generalized Linear Models. New York, Chapman and Hall, 1989.

McGraw-Hill Encyclopedia of Science Technology , 7, 357. New York, McGraw-Hill Book Company.

MILLER, R.G. Survival Analysis. New York, John Wiley \& Sons, 1981. 
MOOD, A.M.; GRAYBILL, F.A; BOES, D.C. Introduction to the Theory of Statistics, 3a ed., New York, McGraw-Hill, 1974.

NELSON, W. Statistical Methods for Accelerated Life Test Data - The Inverse Power Law Model. General Electric Company, Corporation Research and Development Techinical Information Service Report, 71 - C-011, 1970.

NELSON, W. Analysis of Accelerated Life Test Data - Part I: The Arrehnius Models and Graphical Methods. IEEE Transactions on Electrical Insulation, EI-6, 4 165-181, 1971.

NELSON, W. Analysis of Accelerated Life Test Data - Part II: Numerical Methods and Test Planning. IEEE Transactions on Electrical Insulation, EI-7, 1, 36-55, 1972a.

NELSON, W. Analysis of Accelerated Life Test Data - Part III: Product Comparisons and Checks on the Validity of the Model and Data. IEEE Transactions on Electrical Insulation, EI-7, 2, 99-119, 1972 b.

NELSON, W. Graphical Analysis of Accelerated Life Test Data with Inverse Power Law Model. IEEE Transactions on Reliability, R-21, 1, 2-11, 1972c.

NELSON, W. A Survey of Methods for Planning and Analyzing Accelerated Tests. IEEE Transactions on Reliability, EI-9, 1, 12-18, 1974.

NELSON, W. Accelerated Testing - Statistical Models, Tests Plans and Data Analyses. New York, John Wiley \& Sons, 1990.

NELSON, W.; HAHN, G.J. Linear Estimation of a Regression Relationship from Censored Data - Part I: Simple Methods and Their Application. Technometrics, 14, 2, 247-269, 1972d. 
NIRTSON, W.; HAHN, G.J. Linear Estimation of a Regression Relationship from Censored Data - Part II: Best Unbiased Estimation and Theory. Technometrics, 15, 1, 133-150, 1973.

PRESS, S.J. Bayesian Statistics: Principles, Models and Applications. New York, John Wiley \& Sons, 1989.

PROSCHAN, F.; SINGPURWALLA, N.D. Accelerated Life Testing - A Pragmatic Bayesian Approach. Optimizing Methods in Statistics. Academic Press, 385-401, 1979.

RODRIGUES, J. Bayes Predictive Likelihood Function for the Accelerated Life Tests via the Orthogonal Parameters. São Carlos, ICMSC-USP, 1991, 4 p. (Notas do ICMSC-USP, 84).

RODRIGUES, J.; ACHCAR, J.A.; LOUZADA NETO, F. A Bayesian Analysis of the Accelerated Life Tests via the Orthogonal Parameters. São Carlos, ICMSC-USP, 1990, s.p. (Notas do ICMSC-USP, 75).

SHAKED, M.; SINGPURWALLA, N.D. Nonparametric Estimation and Goodness-of-Fit Testing of Hypotheses for Distributions in Accelerated Life Testing. IEEE Transactions on Reliability, R-31, 1, 69-74, 1982.

SHAKED, M.L ZIMMER, W.J.; BALL, C.A. A Nonparametric Approach to Accelerated Life Testing. Journal of the American Statistical Association, 74, 367, 694-699, 1979.

SINGPURWALLA, N.D.; GOLDSCHEN, D.Y. Inference from Accelerated Life Tests Using Eyring Type Reparametrization, Naval Research Logistics, 22(2), 289-296, 1975. 
S.PROTT, D.A. Normal Likelihood and Their Relation to Large Sample Theory of Estimation. Biometrika, 60, 3, 457-465, 1973.

SPROTT, D.A. Maximum Likelihood in Small Samples: Estimation in the Presence of Nuisance Parameters. Biometrika, 67, 3, 515-523, 1980.

THOMAS, R.E. When is a Life Truly Accelerated? Electronic Design, 64-70, 1964.

TIERNEY, L.; KADANE, J.B. Accurate Approximations for the Posterior Moments and Marginal Densities. Journal of the American Statistical Association, 81, 82-86, 1986.

YUM, B.; KIM, S. Development of Life-Test Sampling Plans for Exponential Distributions Based on Accelerated Life Testing. Communications in Statistics. Part A: Theory and Methods, 19(7), 2735-2745, 1990. 